\title{
Uncertainty Quantification in Non-linear Seismic Wave Propagation
}

\author{
by

\section{Chaitanya Raj Goyal}

\begin{abstract}
A thesis submitted to the Faculty of Graduate and Postdoctoral Affairs

in partial fulfilment of

the requirements for the degree of
\end{abstract}

\section{Master of Applied Science}

in

Civil Engineering

Carleton University

Ottawa, Ontario, Canada

January 2017

The Master of Applied Science in Civil Engineering is a joint program with the University of Ottawa, administered by the Ottawa-Carleton Institute for Civil Engineering

\section{Copyright (C)}

2017 Chaitanya Raj Goyal

All rights reserved 


\section{Abstract}

Quantification of uncertainty has become indispensable in contemporary science and technology. Over the past few decades, a framework has been developed which aims at recognizing and quantifying the sources of uncertainty, and understanding their effects on the output of computational models. At the core of this framework lies a computationally efficient and versatile tool based on the polynomial chaos expansion (PCE), which is frequently used in a myriad of research fields. For seismic waves propagating through a two-dimensional soil medium defined by stochastic physical parameters, the effectiveness of non-intrusive polynomial chaos is investigated as a way to characterize the stochastic aspects of wave propagation. The methodology is illustrated with three numerical examples with stochastic constitutive properties, namely (a) a linear elastic 2-D homogeneous soil medium, (b) a linear elastic 2-D layered homogeneous soil profile, (c) a non-linear elasto-plastic 2-D homogeneous soil medium. The results show a considerable difference in the deterministic and the estimated mean response along with major scatter manifested through standard deviation, which proves why uncertainty quantification is important in the analysis of seismic wave propagation.

In this thesis, the finite element method has been used to model the seismic wave equation based on its variational form. The model has been implemented for a two-dimensional linear elastic as well as an elasto-plastic framework, by use of open source platforms like Gmsh, FEniCS, and ParaView. Plasticity is incorporated through the use of non-associative von Mises and Drucker Prager models with isotropic hardening. The computational model for dynamic elasto-plasticity has been 
validated by comparing the response for a small-scale test problem with that from a published open source code. Through this validation, it has also been demonstrated how the model can be extended to solve a three-dimensional problem. Models for seismic applications usually require large-scale simulations with hundreds of millions of degrees of freedom (dof) capable of capturing a wide range of frequencies or wave numbers. The parallel processing capability of the developed model has been exhibited for solving large-scale simulations by using a mesh with over 100 million dof, partitioning it into 1040 sub-domains, and running it on 1040 processors of Guillimin supercomputer at McGill University. 


\section{Acknowledgments}

I would like to extend my deepest gratitude to all those who have made this thesis possible.

To my supervisors: Dr. Abhijit Sarkar and Dr. Siva Sivathayalan, for their time, unwavering support and encouragement, for the wealth of knowledge that they shared with me, and for driving me harder to push my limits. They guided me very patiently through the basics of all the technicalities that were required to perform

this study. I have been extremely fortunate to have had the opportunity to learn and grow under their supervision.

To my exceptional colleagues: Ajit Desai, Sandip Chajjed, Philippe Bisaillon, Rimple Sandhu, Leandro Costa, Perry Natchieppan, Thavakumaran Shanmugarajah, Sentheepan Thirugnanasampanther, Theenathayarl Thayaparan, and Ibrahim Shaheen, for sharing their knowledge, experiences and time with me, for helping and motivating me when I was struggling, and for being good friends. Their contribution to this thesis cannot be quantified.

To Dr. Chris Pettit and Dr. Mohammed Khalil, for guiding me through their helpful comments and advice in the weekly meetings, for taking the time to go through parts of this manuscript, and for sharing their rich and diverse experiences which widened my horizons and knowledge.

To Dr. Kristian Olgaard, Hernan Mella, Dr. Tianyi Li, Dr. Emek Abali and 
Dr. Steven Vandekerckhove for helping with their insightful comments, sharing knowledge and providing selfless guidance. To my thesis committee: Dr. Magdi Mohareb and Dr. Shawn Kenny, for their insightful comments and encouragement.

To Mr. and Mrs. Kochar who funded the Kochar family scholarship, of which I am a grateful recipient.

To my family for the continuous support, unconditional love, and constant prayers. I am forever grateful to them because they make me what I am and help me achieve my goals. I am indebted to them for life.

Above all, to the Almighty God, who is the Center of knowledge and creator of all things. 


\section{Table of Contents}

Abstract $\quad$ ii

Acknowledgments $\quad$ iv

Table of Contents vi vi

List of Tables $\quad$ ix

List of Figures $\quad$ x

List of Acronyms $\quad$ XV

1 Introduction 1

1.1 Motivation . . . . . . . . . . . . . . . . 1

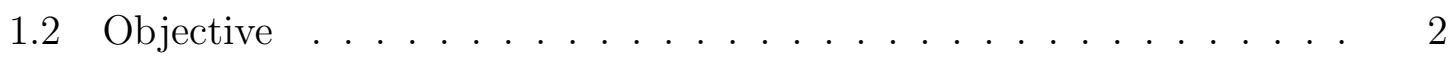

1.3 Thesis Overview . . . . . . . . . . . . . . . 3

2 Literature Review $\quad 6$

2.1 The Wave Equation . . . . . . . . . . . . . . . 6

2.2 Non-linear Seismic Wave Propagation . . . . . . . . . . . . . . . 9

2.3 Uncertainty Quantification in Wave Modelling . . . . . . . . . . . 11

3 Wave Propagation Model $\quad 18$

3.1 Fundamentals of Finite Element Approach . . . . . . . . . . . . . . . 18

3.2 Static Linear Elasticity . . . . . . . . . . . . . . . . . . . 19

3.2.1 Governing Equations of Elastostatics . . . . . . . . . . . 20

3.2.2 Boundary Conditions . . . . . . . . . . . . . . . 25 
3.2 .3 Strong Form to Weak Form . . . . . . . . . . . . . 26

3.3 Acoustic Wave Equation . . . . . . . . . . . . . . . 28

3.3 .1 Semidiscretization of Weak Form . . . . . . . . . . . 30

3.3.2 Numerical Methods for Time Discretization . . . . . . . . . 31

3.4 Elastic Wave Equation . . . . . . . . . . . . . . . . . . 34

3.5 Elasticity to Classical Plasticity . . . . . . . . . . . . . . . . 38

3.6 Theory of Plasticity . . . . . . . . . . . . . . . . . . . 40

3.6.1 Rate dependence in Plasticity . . . . . . . . . . . . . . 40

3.6.2 Understanding Plasticity Models . . . . . . . . . . . . . . 42

3.6.3 Computational Plasticity . . . . . . . . . . . . . . . . . . . 59

3.6.4 Finite Element Method: Weak form . . . . . . . . . . . . 60

3.6.5 Large-scale simulation using high performance computing . . . 72

4 Uncertainty Quantification $\quad 74$

4.1 Monte Carlo Method . . . . . . . . . . . . . . . . . . . 74

4.1 .1 Application . . . . . . . . . . . . . . . 75

4.2 Polynomial Chaos Expansion _ . . . . . . . . . . . . 77

4.2 .1 Orthogonal Polynomials . . . . . . . . . . . . . 78

4.2.2 Galerkin projection and Probability spaces . . . . . . . . 80

4.2.3 One Dimensional Polynomial Chaos Expansion _ . . . . . . 81

4.2.4 Multi-Dimensional Polynomial Chaos Expansion . . . . . . . . 82

4.2.5 Methods for non-intrusive implementation of PCE . . . . . 85

4.2 .6 Why use PCE? . . . . . . . . . . . . . . . . . 93

4.3 Application of non-intrusive PCE to wave propagation $\ldots \ldots . . .99$

4.3.1 Case 1: Linear elastic wave propagation in homogeneous media 100

4.3.2 Case 2: Linear wave propagation in layered piecewise homogeneous media . . . . . . . . . . . . . . . . . . 111 
4.3.3 Case 3: Non-linear elasto-plastic wave propagation in homogeneous media . . . . . . . . . . . . . . . . 113

5 Numerical Application: Results 134

5.1 Concluding Remarks . . . . . . . . . . . . . . . . . . . . . 134

5.2 Future Research . . . . . . . . . . . . . . . . . . . . . 136

$\begin{array}{ll}\text { List of References } & 137\end{array}$ 


\section{List of Tables}

1 Relationship between the type of Wiener-Askey polynomial [1] and the pdf of uncertain inputs . . . . . . . . . . . . . . 80

2 Bivariate Hermite Polynomials for 2-D and total order $p=3[2]$. . 83

3 Multivariate Hermite Polynomials for 3D and total order $p=2 \ldots$. . 84

$4 \quad$ Values of mean and COV for parameters of the linear elastic model . 101

5 Values of mean and COV for parameters of the layered linear elastic model ........................... 112

6 Values of Mean and COV for parameters of the Drucker Prager constitutive model . . . . . . . . . . . . . . . . . . . . . . . . . . . 124 


\section{List of Figures}

$1 \quad$ 2-D domain $\Omega$ partitioned into finite triangular elements using Gmsh $\quad 19$

2 A 3-D carved cube created using Gmsh . . . . . . . . . . . . . . 20

3 Notation for stress components . . . . . . . . . . . . . . . . 22

4 A 3-D domain $\Omega$ clamped at one end, deforming (units in $m$ ) under the influence of self weight and an external force applied at the opposite end 27

5 Magnitude of acoustic waves in a homogeneous isotropic elastic medium 34

6 Magnitude of acoustic waves in a homogeneous anisotropic medium where the wave speed $c$ varies spatially as $2 \sin (x)+y \ldots . . . . .35$

7 1-D wave at time $0.05 s$ propagating on a $10 m$ long bar . . . . . . 36

8 Magnitude of elastic waves in a homogeneous isotropic medium $(1.2 \mathrm{~km}$ wide and $0.6 \mathrm{~km}$ deep), at different time frames . . . . . . . . . . 38

9 (a) Non-linear elastic and elasto-plastic; (b) Linear elastic-perfectly plastic; (c) elasto-plastic with strain hardening [3] . . . . . . . . . . . 40

10 A plane strain element in the $x-y$ plane . . . . . . . . . . . . . 46

11 Yield surface in 2-D stress space [4] . . . . . . . . . . . . . . . . 47

12 Evolution of isotropic and kinematic hardening with loading [5] . . . 50

13 von Mises yield surface in 3-D stress space . . . . . . . . . . . . . 53

14 Isotropic hardening for von Mises yield surface with typical corresponding loading-unloading behavior . . . . . . . . . . . . . . 53

15 Drucker-Prager and Mohr-Coulomb Yield Surfaces . . . . . . . . . . . 54

16 Schematic of return mapping . . . . . . . . . . . . . 60

17 Magnitude of waves propagating in a homogeneous isotropic elastic (left) and elasto-plastic Drucker Prager (right) medium for a weak source 67 
18 Magnitude of waves propagating in a homogeneous isotropic elastic (left) and elasto-plastic Drucker Prager (right) medium for a source of medium strength . . . . . . . . . . . . . . . . . 68

19 Magnitude of waves propagating in a homogeneous isotropic elastic (left) and elasto-plastic Drucker Prager (right) medium for a strong

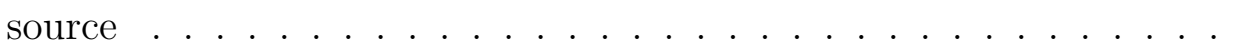

20 Comparison of Deformation: 1) Response of VM-KH model from referred code (left); 2) Response of developed DP-IH model (right) . . . 71

21 Magnitude of displacement shown for a large-scale simulation result (for a Drucker-Prager plasticity model) . . . . . . . . . . . . . 73

22 MC simulation vs. analytical solution for Eq. $98 \ldots \ldots \ldots \ldots$

23 PCE coefficients for the case of a single random variable with $2 n d$ order expansion, on a 1D mesh . . . . . . . . . . . . . . 92

24 4th PCE coefficient $a_{3}$ (sampling-based) for the case of two random variables with $3 r d$ order expansion, on a 1D mesh . . . . . . . .

25 PCE coefficients $a_{4}, a_{5}, a_{6}$ (sampling-based) for the case of two random variables with $3 r d$ order expansion, on a 1D mesh . . . . . . . 95

26 PCE coefficients $a_{7}, a_{8}, a_{9}$ (sampling-based) for the case of two random variables with $3 r d$ order expansion, on a 1D mesh . . . . . . . 96

27 PCE coefficients $a_{1}, a_{4}$ (sampling-based) for the case of two random variables with $2 n d$ order expansion, on a $2 \mathrm{D}$ mesh $\ldots \ldots \ldots$. . . 97

28 PCE coefficients $a_{4}, a_{5}$ (sampling-based) for the case of two random variables with $2 n d$ order expansion, on a 2D mesh . . . . . . 98

29 Magnitude of waves propagating in a homogeneous elastic soil medium: mean $\left(a_{0}\right)$ and deterministic output at $t=1.2 \mathrm{~s} \ldots \ldots \ldots \ldots$

30 Magnitude of waves propagating in a homogeneous elastic soil medium: mean $\left(a_{0}\right)$ and deterministic output at $t=1.9 \mathrm{~s} \ldots \ldots \ldots$ 
31 Magnitude of waves propagating in a homogeneous elastic soil medium: mean $\left(a_{0}\right)$ and deterministic output at $t=2.5 s \ldots . . . . . . .104$

32 1st order PCE coefficient $a_{1}$ : Magnitude plots (left) and Glyph plots

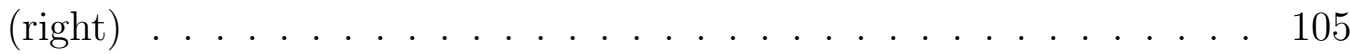

33 2nd order PCE coefficient $a_{4}$ : Magnitude plots (left) and Glyph plots (right) . . . . . . . . . . . . . . . . 106

34 2nd order PCE coefficient $a_{5}$ : Magnitude plots (left) and Glyph plots (right) . . . . . . . . . . . . . . . . 107

$353 r d$ order PCE coefficient $a_{8}$ : Magnitude plots (left) and Glyph plots (right) . . . . . . . . . . . . . . . . 108

$363 r d$ order PCE coefficient $a_{9}$ : Magnitude plots (left) and Glyph plots

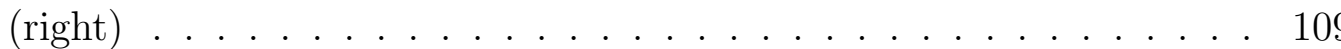

37 Standard deviation for the linear elastic model (2 random variables and $3 r d$ order expansion) . . . . . . . . . . . . . . . . . . . . 110

38 Mesh for layered soil profile . . . . . . . . . . . . . . . . . . . . . 112

39 Magnitude of waves propagating in a layered homogeneous elastic soil medium: mean $\left(a_{0}\right)$ and deterministic output at $t=0.016 \mathrm{~s} \ldots . . .114$

40 Magnitude of waves propagating in a layered homogeneous elastic soil medium: mean $\left(a_{0}\right)$ and deterministic output at $t=0.041 \mathrm{~s} \ldots \ldots 115$

41 Magnitude of waves propagating in a layered homogeneous elastic soil medium: mean $\left(a_{0}\right)$ and deterministic output at $t=0.096 \mathrm{~s} \ldots 116$

42 Magnitude of waves propagating in a layered homogeneous elastic soil medium: mean $\left(a_{0}\right)$ and deterministic output at $t=0.136 \mathrm{~s} \ldots \ldots 117$

43 1st order PCE coefficient $a_{1}$ for case 2: Magnitude plots (left) and Glyph plots (right) . . . . . . . . . . . . . . . . 118

44 1st order PCE coefficient $a_{1}$ for case 2: Magnitude plots (left) and Glyph plots (right) . . . . . . . . . . . . . . . . 119 
45 2nd order PCE coefficient $a_{19}$ for case 2: Magnitude plots (left) and Glyph plots (right) . . . . . . . . . . . . . . . 120

46 2nd order PCE coefficient $a_{19}$ for case 2: Magnitude plots (left) and Glyph plots (right) . . . . . . . . . . . . . . . . . 121

$473 r d$ order PCE coefficient $a_{83}$ for case 2: Magnitude plots (left) and Glyph plots (right) . . . . . . . . . . . . . . . . . . . . 122

48 3rd order PCE coefficient $a_{83}$ for case 2: Magnitude plots (left) and Glyph plots (right) . . . . . . . . . . . . . . . 123

49 Magnitude of waves propagating in a homogeneous elastoplastic Drucker Prager soil medium: mean $\left(a_{0}\right)$ and deterministic output at

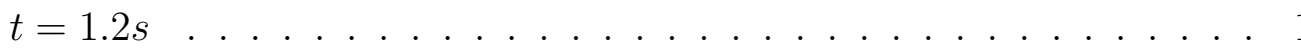

50 Magnitude of waves propagating in a homogeneous elastoplastic Drucker Prager soil medium: mean $\left(a_{0}\right)$ and deterministic output at

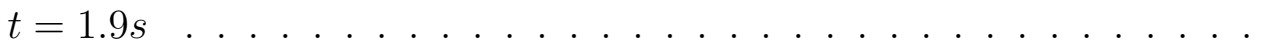

51 Magnitude of waves propagating in a homogeneous elastoplastic Drucker Prager soil medium: mean $\left(a_{0}\right)$ and deterministic output at

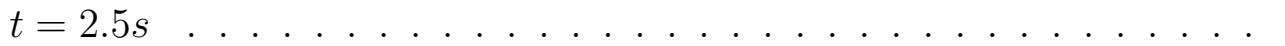

52 1st order PCE coefficient $a_{3}$ for case 3: Magnitude plots (left) and Glyph plots (right) . . . . . . . . . . . . . . . . . 128

53 2nd order PCE coefficient $a_{7}$ for case 3: Magnitude plots (left) and Glyph plots (right) . . . . . . . . . . . . . . . . 129

54 2nd order PCE coefficient $a_{11}$ for case 3: Magnitude plots (left) and Glyph plots (right) . . . . . . . . . . . . . . . . . 130

$553 r d$ order PCE coefficient $a_{15}$ for case 3: Magnitude plots (left) and Glyph plots (right) . . . . . . . . . . . . . . . 131

56 3rd order PCE coefficient $a_{22}$ for case 3: Magnitude plots (left) and Glyph plots (right) . . . . . . . . . . . . . . . . . 132 
57 Standard deviation for the elasto-plastic model (4 random variables and $3 r d$ order expansion) . . . . . . . . . . . . . . . . . . 133 


\section{List of Acronyms}

\begin{tabular}{ll}
\hline Acronyms & Definition \\
\hline \hline CFL & Courant Friedrichs Lewy \\
FDTD & Finite Difference Time-Domain \\
PCE & Polynomial Chaos Expansion \\
MCS & Monte Carlo Simulation \\
FEM & Finite Element Method \\
KLE & Karhunen Loeve Expansion \\
COV & Coefficient of Variation \\
MPI & Message Passing Interface \\
IID & Independent and Identically Distributed \\
pdf & Probability Density Function \\
SFEM & Stochastic Finite Element Method \\
SSFEM & Spectral Stochastic Finite Element Method \\
FPK & Fokker-Planck-Kolmogorov \\
PDE & Partial Differential Equation \\
UQ & Uncertainty Quantification \\
dof & Degrees of Freedom \\
\hline
\end{tabular}




\section{Chapter 1}

\section{Introduction}

\subsection{Motivation}

Numerical modelling and computer simulation of complex systems have made it possible to analyse and design large-scale engineering systems. These computational methods have substantially reduced the requirement for expensive physical experiments, which in many cases are not feasible to perform. It is also recognized that such simulations are often riddled with influence of inherent uncertainties present in the system. These uncertainties may originate from different sources, which may be rooted in different disciplines [6]. The past few years have witnessed a growing emphasis on uncertainty quantification, risk and reliability analysis in civil engineering and especially in geotechnical engineering practice. Seismic modelling is one such field of critical importance which has attracted enormous attention of experts in uncertainty quantification (UQ).

For engineering design of large structural systems, the numerical approximation to the solution of the of wave equations still presents a wide range of problems. Wave propagation simulations for earthquake-induced ground motion have been performed for over three decades to gain a deeper understanding of the spatio-temporal pattern 
of the ground motion in urban regions [7]. A detailed ground motion analysis is critical for seismic design of structures. Simulated ground motions used to design earthquake safe structures are always provided with a certain probability of occurrence. It is also known that system parameters (e.g. material constants, boundary conditions, loading, geometric properties) for such engineering systems can never be determined with absolute precision. Depending on the uncertainties in system parameters and interaction between them, the behaviour of the system may be quite complex [8]. It is therefore plausible to state that these systems are riddled with model errors, numerical errors and parametric uncertainties. In this regard, the inherent uncertainty resulting from random properties of soil deposits through which the seismic waves travel, is generally unaccounted for, since the mainstream models and simulations remain largely deterministic.

In contrast, stochastic models acknowledge the fact that the system properties are not precisely known, but prescribed in a statistical sense. In stochastic systems, system parameters and external input are often modelled as probabilistic quantities (i.e., random variables or processes). To this end, we explore the effects of using a non-intrusive polynomial chaos method to quantify the uncertainty in linear, and nonlinear (elastoplastic) wave propagation models based on the finite element method.

\section{$1.2 \quad$ Objective}

Our objective in this thesis can be classified in two major categories as follows:

\section{Modelling the seismic wave propagation}

- Develop a numerically stable wave propagation model based on variational form of the elastic wave equation for a linear elastic domain, and for a nonlinear elastoplastic domain described by the non-associative von Mises and Drucker 
Prager constitutive models.

- Validate the computational model and run a large scale problem thereby demonstrating the capability of the code to exploit large number of cores in supercomputers.

\section{Quantifying uncertainty in linear and nonlinear seismic wave propagation}

- Implementing a non-intrusive polynomial chaos expansion (PCE) method for both linear and nonlinear seismic wave propagation models based on realistic values of soil parameters obtained in Ottawa valley, and a square mesh of size $1 \mathrm{~km}$.

We aim to execute both the above tasks by making use of the latest advancements in the field of non intrusive spectral projection, latest open source libraries for solving non-linear partial differential equation, in conjunction with the high performance computing (HPC) systems available in Canada.

\subsection{Thesis Overview}

- Chapter 2 presents a literature review on the modelling of wave equation in general, and focusses on the seismic wave propagation in particular. In addition to that, UQ methods for uncertain dynamical systems are thoroughly discussed with a focus on wave propagation. Successful existing methods are examined, and it is observed that most of the research focused in UQ for seismic wave propagation is based on small scale 1D simulations. Many studies have also been conducted using commercial software which becomes fairly expensive for running large-scale parallel simulations. Other studies which have utilised open source libraries have not demonstrated parallel scalability for performing two 
or three-dimensional large scale simulations (for eg. [9]). The chapter also discusses a popular UQ method for dealing with wave propagation problems. The findings constitute the guidance upon which non-intrusive, general-purpose PCE method is implemented.

- Chapter 3 focuses on modelling the linear and elastoplastic wave propagation in time domain using latest open source tools. The chapter discusses the basic theory of elasticity, and plasticity, along with the von Mises and Drucker Prager constitutive models, to provide the readers with a preliminary understanding of the physics of the problem. Also, the variational formulation is derived for the linear and elstoplastic models by discretizing the governing PDEs in space and time. An implicit Newmark Beta method has been used for time integration. The chapter also contains validation efforts for the proposed computational model, and demonstrates its parallel scalability for simulating large scale wave propagation problems.

- Chapter 4 provides the general mathematical foundation of generalized PCE and all the aspects associated with their practical computation. MATLAB codes for simple problems are provided throughout the chapter, to enable easy understanding of the PCE methodology for scalar and vector valued functions.

- Chapter 5 investigates the effectiveness of the non-intrusive PCE method to quantify uncertainty in linear elastic, and elasto-plastic wave propagation problems for three different cases. The first and second order moments are plotted along with other important statistical characteristics of the solution. Results are discussed, conclusions are drawn and future research directions are identified.

This study focuses on applications of PCE in two-dimensional linear and nonlinear seismic wave propagation. However, the methods can be extended and used in 
various contexts such as acoustic, and water wave propagation. More importantly, the implemented strategy appears to be a promising research direction which differs considerably from existing approaches and also sets the foundation for use of latest open source tools with extensive potential for high performance computation. 


\section{Chapter 2}

\section{Literature Review}

This chapter provides a brief overview of the relevant literature. While the review is by no means exhaustive and only pertains to the investigation undertaken in this thesis, it is hoped that the content will be sufficiently detailed to provide the reader with an overall appreciation of the topic.

\subsection{The Wave Equation}

The wave equation is difficult to solve owing to the persistence of sharp features in its solutions which can reflect off boundaries. This complicates both analytical and numerical solution methods because the solution will be smooth only if the initial conditions are smooth [10].

The body waves that propagate in an elastic medium are of three types, i.e., P, $\mathrm{SH}$, and SV waves. Here SV and SH represent shear waves polarised in the vertical and horizontal plane respectively, while $\mathrm{P}$ waves are the compressional waves that are longitudinal in nature. The purpose of seismic modelling is to simulate the wave equation such that the prominent features of all body waves are well captured. This can be performed using different forms of wave equation like the acoustic or elastic 
wave equation. While the acoustic wave equation can only be used to model the propagation of $\mathrm{P}$ waves, the elastic wave equation can model both $\mathrm{P}$ and $\mathrm{S}$ waves. In acoustic wave equation, the dependent variable is a scalar field. Therefore, it is also known as scalar wave equation. The acoustic approximation considers the $\mathrm{P}$ wave as the dominant type of wave while ignoring the shear effects in wave propagation, which can be an acceptable approximation in certain layered soil media [11]. De Basabe Delgado [12] and Mishra et al. [13] provide examples of modelling earthquake waves as acoustic waves. For linearly elastic model, a transient incident plane SH-wave modelled as acoustic wave has also been widely used in the literature (e.g. Bielak et al. [14], Yeh and Rehman [15], Xu [16]).

The elastic wave equation is the most accurate option to model seismic wave propagation but it requires higher computational effort because the solution, 'displacement' is a vector field. Seismic modelling using Navier's equation of linear elastodynamics for isotropic and homogenous media has been performed by Bielak et al. [7], Xu [16], Furumura and Chen [17], and Bao et al.[18].

Multiple numerical methods have been reported in literature for wave propagation and ground motion analysis. The finite element method, finite volume method, finite difference method, boundary element method, and the spectral element method are commonly used. The first numerical methods employed for solving the wave equation were finite difference methods. Amongst the first finite difference-time domain (FDTD) techniques used was the non-dissipative Yee scheme [19], which is an explicit algorithm based on central differences in space (staggered grid) and a staggered leapfrog time stepping scheme. From a computational perspective, a Cartesian grid is also quite efficient for solving wave propagation problems. The finite difference technique has therefore been quite popular for its ease of implementation, accuracy and low memory needed per grid point [e.g., Furumura and Chen [17]; Kozdon et al. [20]; 
Graves [21]]. However, the conventional finite difference method is not suitable for heterogeneous anisotropic media due to its reliance on structured grids which cannot adapt to the local wavelengths of propagating waves. This makes the grid resolution dependent on shortest wavelengths, which results in over-refined grids in stiffer regions and this makes the time step for numerical integration very small to satisfy the CFL (Courant-Friedrichs-Lewy) stability condition in the over-refined stiff regions [7]. Also, it becomes difficult to deal with complex geometries and irregular boundaries while using this method. For example, when solving problems of scattering by an obstacle, it creates numerical diffraction when the boundary of the obstacle does not fit the mesh grid, unless an extremely fine mesh resolution is implemented [22].

Finite volume and boundary element method have also been used in the past to solve wave propagation problems, for example, Mishra et al. [13], Dumbser et al. [23], Zhang and Chopra [24]. While the boundary element method is capable of providing a complete solution in terms of just the boundary values (which considerably reduces the modelling effort), it is not the best choice for modelling heterogeneous basins since it demands piecewise-homogeneity of the domain [7].

A more popular option is to implement the weak (variational) form of the equation using the finite element method. Both the finite element method and spectral element method are based on the variational approach. Not only do they make it easier to work with complex geometries because the free surface condition is automatically taken into account, but they also provide considerable improvement in terms of computational efficiency and accuracy [25]. The finite element method is also more appropriate than the finite difference technique for heterogeneous media because it is capable of adapting the mesh to local features of the solution thereby eliminating over-refinement of grids and allowing for larger time steps satisfying the CFL condition. The applicability of large-scale finite element simulations in earthquake wave 
modelling has been demonstrated extensively in literature (e.g. [7, 12, 14, 16, 18, 2528]). The FEM is therefore used in this research for its ability to handle complex geometries, and efficiently resolve wide range of spatial and temporal scales.

\subsection{Non-linear Seismic Wave Propagation}

In the past few decades, earthquake generated ground motion simulations have generally been implemented through two broad classes of soil models: equivalent linear models based on an elastic or visco-elastic constitutive models, and non-linear models based mainly on elasto-plastic constitutive models. The former is more popular in current practice. Equivalent linear elastic models are quite straight forward and perform reasonably well at describing moderate non-linear behaviour. However, this approximation holds good only for weak loadings conditions ([29], [30]) because equivalent linear models are incapable of accurately representing the observed plastic strain induced in soil at yield point [16]. The late 1960s witnessed an increasing emphasis on consideration of non-linearity in seismic wave propagation research and since early 1970s, numerous programs and softwares (1-D/2-D/3-D) for ground motion analysis have been under development, for e.g. Cyberquake [31], SHAKE [32] among others. While the 1-D equivalent linear simulators like SHAKE are capable of yielding reasonable estimates of non-linear effects under vertically incident seismic excitation for horizontal layers, they cannot capture the effects of surface waves and complex basin geometry. However, other commercial software like FLAC, PLAXIS and Cyberquake which are capable of running non-linear seismic analysis have also gained considerable popularity in last decade.

The response using both the techniques is different for certain type of loadings because of the methodology involved. The equivalent linear method evaluates soil 
response in an iterative manner where initially the soil properties are chosen in accordance with the average strain that occurs at each depth in the model during excitation, and eventually the model response is calculated. The process is repeated if the estimated strains are considerably different from the trial values [33]. This method often overestimates the seismic response [28]. Also, as noted earlier, neither can it predict the plastic behaviour of soil nor can it capture the important characteristics of ground motion under strong seismic loading. However, the non-linear approach uses the current strain to modify the shear modulus at every time step. This fact reasonably approximates the non-linear stress-strain relationship.

One of the major issues that delayed emphasis on the study of non-linear seismic response was scarce evidence of non-linearity in the observed ground motion at locations apart from the liquefied sites. However, accelerogram recordings of many earthquakes have shown that non-linear soil behaviour affects the seismic motion of deposits near ground surface, thereby resulting in decrease of shear wave velocity, increase of soil damping with increasing load [34], irreversible settlements, shift of the predominant frequency towards lower frequencies, and sometimes increase in pore pressure leading to liquefaction [31]. Soil non-linearity in dynamic analysis has been observed by many experimentalists in the past like Vucetic and Dobry [35] and Seed and Idriss [36]. Finn [37] and Seed and Idriss [38] are popular references that discuss non-linear soil amplification.

Despite the availability of aforementioned software and literature related to nonlinear seismic analysis, there are only a few references describing non-linear simulations with complex constitutive models in geological structures, using finite element method for realistic applications. Xu [16], Dupros et al. [25] and Xu et al. [28] are some popular references that have implemented the elasto-plastic constitutive model based on finite element software. Another popular approach is to use a visco-plastic 
constitutive model, the use of which has grown significantly in recent years by the soil dynamics scientific community (e.g., [39], [40], and [41]). It also seems to be one of the most promising models in order to accurately study the phenomena of non-linear seismic wave propagation.

\subsection{Uncertainty Quantification in Wave Modelling}

Uncertainties can be commonly categorized as epistemic or aleatory uncertainties [42]. Those that arise due to lack of information are called epistemic uncertainties, while those that exist due to inherent randomness of nature are called aleatory uncertainties (e.g. uncertain loading). It is a very challenging task to reduce the influence of the latter, but there exist highly developed mathematical theories dealing with such problems [8]. On the other hand, the effect of epistemic type of uncertainty can be decreased by gathering more information. Both of the aforementioned uncertainties arise while solving wave propagation problems, and stochastic models with rich theoretical and computational structure can be used to treat them. Several numerical methods are available to tackle the time evolution of stochastic systems described by PDEs containing random coefficients. Each of them is based on a different methodology to capture the underlying randomness [43].

Heterogeneities present in soil media cause variability in speed of waves propagating through it. Non-linear physical processes drive this medium variability. Stochastic analysis of seismic wave propagation deals with both - uncertainty quantification (UQ) of soil parameters and propagation of such uncertainties through the governing wave equation. The uncertain soil properties can be modelled by using stochastic fields or in simple cases random variables, depending on whether they vary spatially in continuum [44]. Many efforts have been made over the past few decades to study 
wave propagation in random media, using a variety of methods. A strain compatible non-linear elastic model for 1-D scalar wave propagation was used by Yeh and Rahman ([15], [45]) with a spatially varying shear modulus and an input motion treated as stochastic process. Amongst the four methods implemented in the study, i.e., (i) direct Monte Carlo simulation, (ii) perturbation method, (iii) Neumann expansion based spectral decomposition, and (iv) polynomial chaos expansion (PCE) based decomposition, it was observed that the Monte Carlo method is the most dependable and versatile method for stochastic analysis of seismic ground motion. Nour et al. [46] also carried out probabilistic seismic response analysis using Monte Carlo simulations with deterministic FEM solver - FLUSH [47]. Hacefendiolu et al. [48] investigated the effect of lognormally distributed shear modulus on the dynamic response of a dam foundation system subjected to non-stationary random excitation, using the Monte Carlo method. For an elastic domain, the study was based on an equivalent linear method.

Monte Carlo simulation (MCS) is the most versatile method and convenient to implement in uncertainty quantification. However, MCS becomes computationally inefficient as a large number of samples are needed to produce results [49]. Given that the seismic wave propagation problems typically involve large-scale computational models, which exhibit complexity and non-linearity even in the deterministic case, MCS based reliability analysis would require many realizations (samples), thereby making it impractical [50]. There are improved sampling methods such as Latin hypercube sampling [51] and multi-level MCS [52], but they have theoretical and implementational difficulties.

Popular alternatives include the stochastic finite element methods (SFEM), which implement the finite element method (FEM) for analysing systems with parametric 
uncertainties. Aquino [53], and Stefanou [54] are excellent references which provide a detailed review of SFEM. However, some of these formulations too have their shortcomings. For example, perturbation methods do not allow high parametric uncertainties [55], and also suffer from poor accuracy and convergence issues. Soil has material parameters with large coefficient of variation (COV) and therefore this may not be an appropriate method for solving problems in seismic wave propagation [8].

The method implemented in this study is based on polynomial chaos expansion (PCE), which is a method for the spectral representation of uncertainty, that discretizes the stochastic problem using a set of random variables. In PCE, any random function (stochastic process) can be approximated as an expansion in orthogonal polynomials of a set of known random variables [56]. Ghanem and Spanos [57] first applied this to the finite element method based on Wiener-Hermite Chaos, and it was later extended to the Wiener-Askey PCE in [1]. PCE allows to cheaply compute stochastic quantities of interest such as mean value, standard deviation, skewness, kurtosis, any higher order statistical moments, and the probability density function (pdf). It is cheaper than MCS in terms of function evaluations, thereby making it an attractive option for solving stochastic FEM systems [58-60].

Since the early 1990's many studies have been conducted on uncertainty quantification in wave propagation but a lot of them have been focused on electromagnetic, shallow water, underwater sound and even cardiovascular pulse waves. Many of these studies [56, 61-65], suggested that polynomial chaos might be useful for incorporating variability into wave propagation problems. In 2002, Ghiocel and Ghanem [66] introduced a procedure for the probabilistic analysis of soil structure interaction problem, in which they modelled the free field input motion along with local soil parameters as stochastic processes. The shear modulus and material damping were assumed to be random functions of the shear strain [66]. However, the ground motion analysis was 
performed using SHAKE 91 [32], which is a 1-D program based on equivalent linear method. The proposed approach was based on PCE representation of the stochastic solution. In 2013, Al-Bittar et al. [67] also studied probabilistic seismic behaviour considering the shear modulus as a one-dimensional, vertically varying, non-Gaussian random field, and using both Monte Carlo Simulation and Sparse PCE methodology. However, the model was elastic and the deterministic analysis was performed using finite difference based FLAC 3-D. Another good reference demonstrating the use of seismic modelling and PCE is Wang and Hao [68].

PCE based stochastic finite element method can be carried out using the intrusive and non-intrusive approaches. As the name indicates, the intrusive approach (also known as the spectral stochastic finite element method or SSFEM) seeks to modify the deterministic model. This may prove to be non-trivial and time consuming for many computational models such as the one considered in this thesis. On the other hand, the non-intrusive approach treats the deterministic code like a 'black box', collecting the outputs using sampling for UQ. Both the approaches have been widely implemented for UQ in wave propagation. Application of SSFEM to the three-dimensional wave propagation analysis was researched by Honda and $\mathrm{Mu}-$ rakami [69] through implementation of Karhunen-Loeve (KL) expansion for spatial distribution of uncertain parameters and PCE for the stochasticity of solution, using parallel computing. The shear stiffness was however, taken to be independent of the strain. In 2015, Wang and Sett [70] presented a time-domain SFEM, based on an intrusive stochastic Galerkin approach, for simulating 1-D elastic wave propagation with uncertainty in both material parameters and forcing function.

At this point, your attention is focussed on to the fact that most of the studies listed in this section involve only the problems dealing with geometrically linear or non-linear problems with linear elastic material. In reality however, soil properties are 
best represented by elasto-plastic constitutive models (i.e., material non-linearity). The few existing studies that have focused on uncertainty quantification in elastoplastic media are discussed in the next section. Also, it must be noted that most (if not all) of the aforementioned studies are either based on expensive commercial software, or old open source codes which are not very effective in exploiting modern high performance computing systems. Efforts to quantify uncertainty in seismic modelling problems must be accompanied by the promise of parallel computing in order to tackle large-scale problems in 2-D and 3-D. Also, considerable financial savings can be made if the computational model is based on latest open source tool kits built with easy to use programming languages and state-of-art high performance computing tools.

\section{Stochastic analysis of Dynamic Elasto-Plastic problems}

To solve a dynamic problem like seismic wave propagation, it is better to implement an elasto-plastic model than use the equivalent linear approach, since the former represents a rational mechanistic process. However, a major obstacle in its implementation arises in the form of difficulty in parameter identification, in conjunction to the lack of knowledge of soil properties [71].

Anders and Hori [72] used SSFEM with KLE and PCE, and applied it to a fault formation problem for softening elasto-plastic bodies with a random Young's modulus. Later, Anders and Hori [73] used the perturbation approach to develop a 3-D stochastic finite element method for elasto-plastic bodies. However, while solving problems related to soil, the perturbation approach suffers from defects highlighted in the previous section. A detailed discussion on the limitations of the aforementioned works was presented in [8]. To transcend the limitations posed by MCS and perturbation approach, Jeremic et al. [8], [74] proposed an Eulerian-Lagrangian form of the Fokker-Planck-Kolmogorov (FPK) equation corresponding to a general 1-D 
elasto-plastic constitutive rate equation. The usefulness of the FPK equation based approach was supported by establishing that it converts the original stochastic nonlinear constitutive equation into a linear deterministic PDE. The proposed SSFEM was therefore capable of carrying out probabilistic integration at the constitutive level. Caballero and Modaressi [71] conducted a Monte Carlo based probabilistic analysis to obtain the uncertainties in seismic response using a 2-D coupled FEM software GEFDyn [75] for an elasto-plastic multi-mechanism Hujeux model. In 2012, Sett et al. [9], used the previously developed FPK based stochastic elasto-plastic FEM computational framework along with KLE and PCE [74] for discretizing (in stochastic and spatial dimensions) the soil properties and output (displacement) random fields, respectively. They implemented the method to perform a 1-D site response analysis on a site in northern California, where the rock motion was propagated through the top 8.5m of subsoil. In 2014, Sadrinezhad [76] built upon the work of [8], [74], and [9] to extend the the existing one-dimensional FPK framework for probabilistic constitutive modelling of soils, to multi-dimension. The study focused on developing algorithms based on the Fourier spectral approach for solving a class of FPK PDEs that arise in probabilistic elasto-plasticity. The uncertain constitutive response of linear elastic and von Mises elastic-perfectly plastic soils was simulated by observing the uniaxial and multiaxial, monotonic and cyclic behaviour of soils subjected to an unconfined compression test and to an unconsolidated undrained triaxial test.

By now it is evident that the intrusive method has been widely used in the literature. However, it comes with its hardships. In 2016, a detailed study was performed by Van [77] on PCE for uncertain dynamical systems with applications in earthquake engineering. The study claimed that the intrusive method is difficult to implement, which is a common situation in practice when handling legacy computer codes. Despite the advantage that the final system of coupled equations obtained using intrusive 
approach has to be solved only once, the resulting equations are considerably more complicated than the original deterministic system of equations. The study also highlighted that significant issues will arise in deriving a suitable system of equations based on the intrusive method for problems involving complex dynamical behaviour.

It therefore becomes necessary to introduce a non-intrusive method which involves repeated simulation of deterministic system as a black-box. Non-intrusive methods only make use of a set of samples obtained by running the numerical model [77]. Acharjee and Zabaras [78] also implemented the non-intrusive PCE method for static elasto-plastic problems, since it is faster than MCS and more convenient to implement than the intrusive method. Therefore, it provides an efficient framework for uncertainty quantification of complex models that have been already tested in the deterministic domain through verified computer codes.

Therefore, in this study our efforts are concentrated specifically in analysing the effectiveness of a robust non-intrusive PCE approach for quantifying uncertainty in FEM based linear elastic and non-linear elasto-plastic 2-D wave propagation problems. 


\section{Chapter 3}

\section{Wave Propagation Model}

The finite element method (FEM) is very useful tool for modelling of physical phenomena due to its unique ability to find approximate solutions to boundary value problems for PDEs. For complex geometrical configurations, where the mesh is unstructured and boundaries are curved, FEM is the most preferred option, as highlighted in the discussion in chapter 2 . We begin this chapter with a brief discussion on the concept of FEM. Thereafter, we revisit the basics of static elasticity and model a linear problem using the weak form of elastostatics. Eventually, we derive the weak form for linear elastodynamics, and elasto-plastic wave propagation and discuss the resulting simulations.

\subsection{Fundamentals of Finite Element Approach}

The FEM discretizes a structure into many small elements connected at nodes, where each node has a certain number of degrees of freedom (dof). Therefore, the representation is in terms of the number of elements, the number of nodes per element, and the number of dof per node. The output quantity is interpolated over a single element from values obtained at the nodes by adopting polynomial interpolation functions. 
By connecting the elements, the unknown quantity can be approximated over the entire system.

Discretization of a domain can be performed through various combinations of element shapes and number of nodes on each element. For instance, line segments in 1-D, triangles and quadrilaterals in 2-D, and tetrahedra, prisms and hexahedra in 3-D. For 2-D domains especially, triangular elements are very good because they can approximate a wide range of geometries while quadrilateral elements have difficulties at corners. We have made use of the open source FEM mesh generator - Gmsh [79] to create all the $1-\mathrm{D} / 2-\mathrm{D} / 3-\mathrm{D}$ meshes used in this research. The concepts described henceforth are based on $[3,80-84]$ and the readers are directed to these references if they wish to explore these concepts in detail.

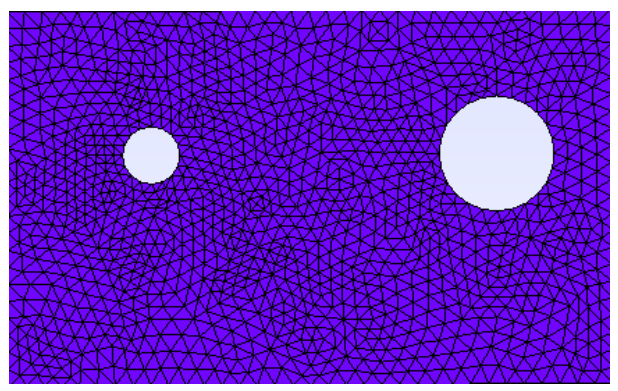

Figure 1: 2-D domain $\Omega$ partitioned into finite triangular elements using Gmsh

\subsection{Static Linear Elasticity}

Deformations of almost all solid materials can be described by the theory of linear elasticity if they are subjected to sufficiently small strains and stresses. Since the strains are small, all the governing equations for linear elastic materials can be linearized, and are therefore relatively easy to solve. For simplicity, we only consider the example of a linear problem in the subsequent section, but it must be noted that the framework also supports the implementation of non-linear problems. 


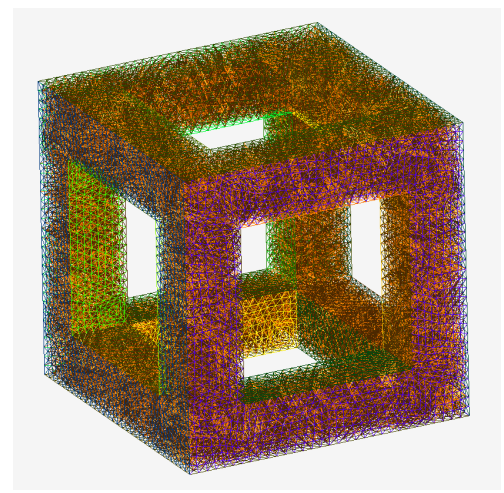

(a) Coarse mesh

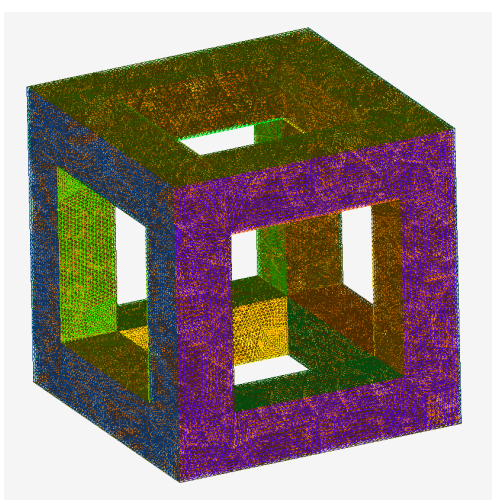

(b) Fine mesh

Figure 2: A 3-D carved cube created using Gmsh

\subsubsection{Governing Equations of Elastostatics}

The equations which govern the linear elastic response of a solid due to external loading, have been summarised below. In the equations that follow, the notation for the position of a point in three-dimensional space is defined by the Cartesian coordinates $\left(x_{1}, x_{2}, x_{3}\right)$. Latin indices $i, j, k, l$ will be used to refer to any one of the values $1,2,3$, so that the symbol $x_{i}$ can represent either one of $x_{1}, x_{2}, x_{3}$.

\section{Strain-Displacement Equations}

When the displacement field is given, the strain field can be obtained from the straindisplacement equations. The strain tensor $\epsilon_{i j}$ in linear elasticity, for a body of volume $V$, is given by [85]:

$$
\begin{aligned}
\epsilon_{i j} & =\frac{1}{2}\left(u_{i, j}+u_{j, i}\right) \\
& =\frac{1}{2}\left(\frac{\partial u_{i}}{\partial x_{j}}+\frac{\partial u_{j}}{\partial x_{i}}\right) \text { in } \quad V \\
\epsilon & =\frac{1}{2}\left(\nabla+\nabla^{T}\right) \cdot u \quad \text { in } \quad V
\end{aligned}
$$


The convenient way to describe the motion of a linear material is through the displacement field $u$ which is the function of Lagrangian coordinates and time. However, we cannot solve for displacements even when the strain field is given, unless the following six strain compatibility conditions are fulfilled [86].

$$
\begin{aligned}
2 \frac{\partial^{2} \epsilon_{12}}{\partial x_{1} \partial x_{2}} & =\frac{\partial^{2} \epsilon_{11}}{\partial x_{2}^{2}}+\frac{\partial^{2} \epsilon_{22}}{\partial x_{1}^{2}}, \\
2 \frac{\partial^{2} \epsilon_{23}}{\partial x_{2} \partial x_{3}} & =\frac{\partial^{2} \epsilon_{22}}{\partial x_{3}^{2}}+\frac{\partial^{2} \epsilon_{33}}{\partial x_{2}^{2}}, \\
2 \frac{\partial^{2} \epsilon_{13}}{\partial x_{1} \partial x_{3}} & =\frac{\partial^{2} \epsilon_{11}}{\partial x_{3}^{2}}+\frac{\partial^{2} \epsilon_{33}}{\partial x_{1}^{2}}, \\
\frac{\partial^{2} \epsilon_{11}}{\partial x_{2} \partial x_{3}} & =\frac{\partial^{2} \epsilon_{12}}{\partial x_{3} \partial x_{1}}-\frac{\partial^{2} \epsilon_{23}}{\partial x_{1}^{2}}+\frac{\partial^{2} \epsilon_{13}}{\partial x_{1} \partial x_{2}}, \\
\frac{\partial^{2} \epsilon_{22}}{\partial x_{1} \partial x_{3}} & =\frac{\partial^{2} \epsilon_{23}}{\partial x_{1} \partial x_{2}}-\frac{\partial^{2} \epsilon_{13}}{\partial x_{2}^{2}}+\frac{\partial^{2} \epsilon_{12}}{\partial x_{2} \partial x_{3}}, \\
\frac{\partial^{2} \epsilon_{33}}{\partial x_{1} \partial x_{2}} & =\frac{\partial^{2} \epsilon_{13}}{\partial x_{2} \partial x_{3}}-\frac{\partial^{2} \epsilon_{12}}{\partial x_{3}^{2}}+\frac{\partial^{2} \epsilon_{23}}{\partial x_{1} \partial x_{3}} .
\end{aligned}
$$

\section{Constitutive Equations}

The stress and strain fields in the body $V$ are connected by the constitutive equations. These PDEs in space and time, enable the modelling of materials as continua. A stress $\sigma$ component within a body can be defined by identifying the plane on which it acts along with the direction of the traction on that plane. The plane is defined by its outward normal, i.e., the $x_{i}$-plane is perpendicular to the direction $x_{i}$. Therefore, the stress component $\sigma_{i j}$ is defined as the component of traction in $j$-direction acting on the $x_{i}$-plane (Fig. 3). The moments acting on the object in Figure 3 are supposed to be in equilibrium and hence require that $\sigma_{i j}=\sigma_{j i}$, and therefore the stress tensor is 

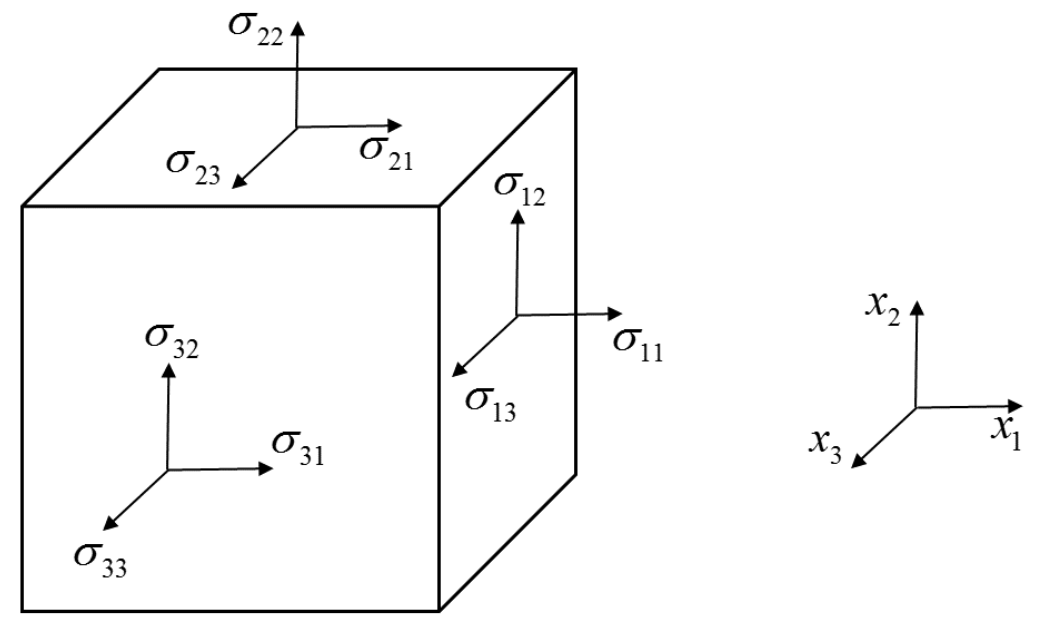

Figure 3: Notation for stress components

symmetric.

$$
\sigma=\left[\begin{array}{ccc}
\sigma_{11} & \sigma_{12} & \sigma_{13} \\
\sigma_{21} & \sigma_{22} & \sigma_{23} \\
\sigma_{31} & \sigma_{32} & \sigma_{33}
\end{array}\right]
$$

The stress-strain relations follow Hooke's law, and may be written in indicial notation as [3]:

$$
\sigma_{i j}=C_{i j k l} \epsilon_{k l}=C_{i j k l} \frac{\partial u_{k}}{\partial x_{l}} \quad \text { in } \quad V
$$

In concise form, the above equations become

$$
\sigma=C \epsilon
$$

Here, tensors $\boldsymbol{\sigma}$ and $\epsilon$ are now reduced to 6 element vectors due to internal symmetry, and $C_{i j k l}$ is called the elasticity tensor of rank four. Due to the inherent symmetric nature of this tensor in addition to the symmetry resulting from the material being 
isotropic, $C_{i j k l}$ can be represented in terms of just two material constants [83]:

$$
C_{i j k l}=\lambda \delta_{i j} \delta_{k l}+\mu\left(\delta_{i k} \delta_{j l}+\delta_{j k} \delta_{i l}\right)
$$

where $\lambda, \mu$ are Lame's constants and $\delta_{i j}$ is the Kronecker delta function. The elastic constitutive law in Eq. 4 then takes the form [83]

$$
\sigma_{i j}=\lambda \epsilon_{k k} \delta_{i j}+2 \mu \epsilon_{i j}=\lambda \delta_{i j} \frac{\partial u_{k}}{\partial x_{k}}+\mu\left(\frac{\partial u_{i}}{\partial x_{j}}+\frac{\partial u_{j}}{\partial x_{i}}\right)
$$

Therefore, the constitutive relations for isotropic materials may be expressed as [84]:

$$
\begin{aligned}
& \sigma_{11}=\lambda \delta_{11}\left(\epsilon_{11}+\epsilon_{22}+\epsilon_{33}\right)+\mu\left(\epsilon_{11}+\epsilon_{11}\right)=(\lambda+2 \mu) \epsilon_{11}+\lambda \epsilon_{22}+\lambda \epsilon_{33} \\
& \sigma_{22}=\lambda \delta_{22}\left(\epsilon_{11}+\epsilon_{22}+\epsilon_{33}\right)+\mu\left(\epsilon_{22}+\epsilon_{22}\right)=(\lambda+2 \mu) \epsilon_{22}+\lambda \epsilon_{11}+\lambda \epsilon_{33} \\
& \sigma_{33}=\lambda \delta_{33}\left(\epsilon_{11}+\epsilon_{22}+\epsilon_{33}\right)+\mu\left(\epsilon_{33}+\epsilon_{33}\right)=(\lambda+2 \mu) \epsilon_{33}+\lambda \epsilon_{11}+\lambda \epsilon_{22} \\
& \sigma_{12}=2 \mu \epsilon_{12} \\
& \sigma_{23}=2 \mu \epsilon_{23} \\
& \sigma_{31}=2 \mu \epsilon_{31}
\end{aligned}
$$

where, the cubical dilation $\left(\epsilon_{11}+\epsilon_{22}+\epsilon_{33}\right)=\left(\frac{\partial u_{1}}{\partial x_{1}}+\frac{\partial u_{2}}{\partial x_{2}}+\frac{\partial u_{3}}{\partial x_{3}}\right)$ and $\epsilon_{i j}$ is as defined in Eq. 1. At this point, it is straight forward to write the corresponding stress-strain 
relations as [84]:

$$
\left[\begin{array}{c}
\sigma_{11} \\
\sigma_{22} \\
\sigma_{33} \\
\sigma_{23} \\
\sigma_{13} \\
\sigma_{12}
\end{array}\right]=\left[\begin{array}{cccccc}
\lambda+2 \mu & \lambda & \lambda & 0 & 0 & 0 \\
\lambda+2 \mu & \lambda & 0 & 0 & 0 \\
& \lambda+2 \mu & 0 & 0 & 0 \\
& & \mu & 0 & 0 \\
& & & & \mu & 0 \\
& & & & & \\
& & & & &
\end{array}\right]\left[\begin{array}{l}
\epsilon_{11} \\
\epsilon_{33} \\
2 \epsilon_{23} \\
2 \epsilon_{13} \\
2 \epsilon_{12}
\end{array}\right]
$$

It is also known that [86]

$$
\begin{array}{r}
\epsilon_{11}=\frac{1}{E}\left[\sigma_{11}-\nu\left(\sigma_{22}+\sigma_{33}\right)\right] \\
\epsilon_{22}=\frac{1}{E}\left[\sigma_{22}-\nu\left(\sigma_{11}+\sigma_{33}\right)\right] \\
\epsilon_{33}=\frac{1}{E}\left[\sigma_{33}-\nu\left(\sigma_{11}+\sigma_{22}\right)\right] \\
2 \epsilon_{23}=\frac{\sigma_{23}}{G}, \quad 2 \epsilon_{13}=\frac{\sigma_{13}}{G}, \quad 2 \epsilon_{12}=\frac{\sigma_{12}}{G}
\end{array}
$$

Here, the engineering constants, Young's modulus $E$ and Poisson ratio $\nu$ are related to the shear modulus $G$ by the expression:

$$
G=\frac{E}{2(1+\nu)}
$$

When the set of Eq. 10 is expressed in matrix form, then inverted and compared with Eq. 9, it can be shown that [81]:

$$
\mu=\frac{E}{2(1+\nu)}, \quad \lambda=\frac{E \nu}{(1+\nu)(1-2 \nu)}
$$




\section{Equilibrium Equations}

In elastostatics, the equilibrium equations are given as:

$$
\sigma_{i j, j}+f_{i}=\frac{\partial \sigma_{i j}}{\partial x_{j}}+f_{i}=0 \quad \text { in } \quad V
$$

where $f$ is the acting body force per unit volume. In tensor notation, it can be written as:

$$
\nabla \cdot \underline{\boldsymbol{\sigma}}+\boldsymbol{f}=0 \quad \text { in } \quad V
$$

\subsubsection{Boundary Conditions}

There exist two types of classical boundary conditions for elastostatics, which connect displacements and fluxes to the given data in order to satisfy boundary compatibility and equilibrium conditions. These are the surface compatibility equations and the surface equilibrium equations. The former, also known as displacement or dirichlet boundary conditions is given as

$$
u_{i}=\hat{u}_{i} \quad \text { on } \quad S_{1}
$$

which implies that the displacement components at points of surface $S_{1}$ of volume $V$ must match the prescribed values. The other type of boundary condition also known as traction or neumann type is given as

$$
\sigma_{i j} n_{j}=\hat{t}_{i} \quad \text { on } \quad S_{2}
$$

where $n_{j}$ represents components of the outward normal $n$ at points on the surface $S_{2}$ where traction is specified. 


\subsubsection{Strong Form to Weak Form}

FEM doesn't actually solve the original equation, but rather uses the "weak or variational form" of the original equation. The governing PDE is known as the "strong form" because it implies that the relationship must satisfy at all points in the domain. However, a "weak form" implies that the relationship (in integral form) is satisfied only in overall sense and is not essentially satisfied on all the points in the domain. The purpose of this variational form is to satisfy the equation in the "average sense," so that we can approximate solutions that are discontinuous or otherwise poorly behaved. Therefore, to solve any PDE the finite element approach requires its reformulation into an equivalent weak form. To compute the finite element solution for elastostatics, we begin by formulating the problem based on Eq. 14 as follows:

Find the displacement field $u$ of an isotropic, homogeneous elastic domain: $\Omega \longmapsto \mathbb{R}^{3}$ such that,

$$
\begin{array}{r}
\nabla \cdot(\sigma(u))+f=0 \quad \text { in } \quad \Omega, \\
u=0 \quad \text { on } \quad \Gamma_{L}, \\
\sigma(u) \cdot \hat{n}=0 \quad \text { on } \quad \partial \Omega
\end{array}
$$

where $\sigma(u)$ is the symmetric Cauchy stress tensor given by [85],

$$
\sigma(u)=2 \mu \epsilon(u)+\lambda \operatorname{tr}(\epsilon(u)) I
$$

$\hat{n}$ denotes unit vector normal to the boundary $\partial \Omega$, $\operatorname{tr}$ denotes the trace, $I$ is the identity matrix and the strain tensor $\epsilon(u)$ is given by,

$$
\epsilon(u)=\frac{1}{2}\left(\nabla(u)+\nabla(u)^{T}\right),
$$


as shown in Eq. 1. The Lame's parameters remain as defined in Eq. 12, and we use $E=210 \mathrm{GPa} ; \nu=0.3$. The discrete variational problem corresponding to Eq. 17 can be obtained by multiplying it with a test function $v$ in a suitable discrete test space $\hat{V}_{h}$ and integrating by parts [85, 87]:

$$
\begin{aligned}
\int_{\Omega}(\nabla v): \sigma(u) d x & =\int_{\Omega} v \cdot f d x & \forall v \in \hat{V}_{h} \\
\text { or } \quad \int_{\Omega} \sigma(u): \epsilon(v) d x & =\int_{\Omega} v \cdot f d x & \forall v \in \hat{V}_{h}
\end{aligned}
$$

where the symbol : represents the Frobenius inner product. For the simulation displayed in Fig. 4, the mesh consisting of tetrahedral (3-D) elements was generated using Gmsh and the finite element code implementing the weak form in Eq. 20 was created in python using the open source computing platform for partial differential equations - FEniCS [85, 88-113]. Lagrange elements (continuous piecewise polynomial functions) of second order were used, and the code was validated based on the results reported in [87] for a similar problem.
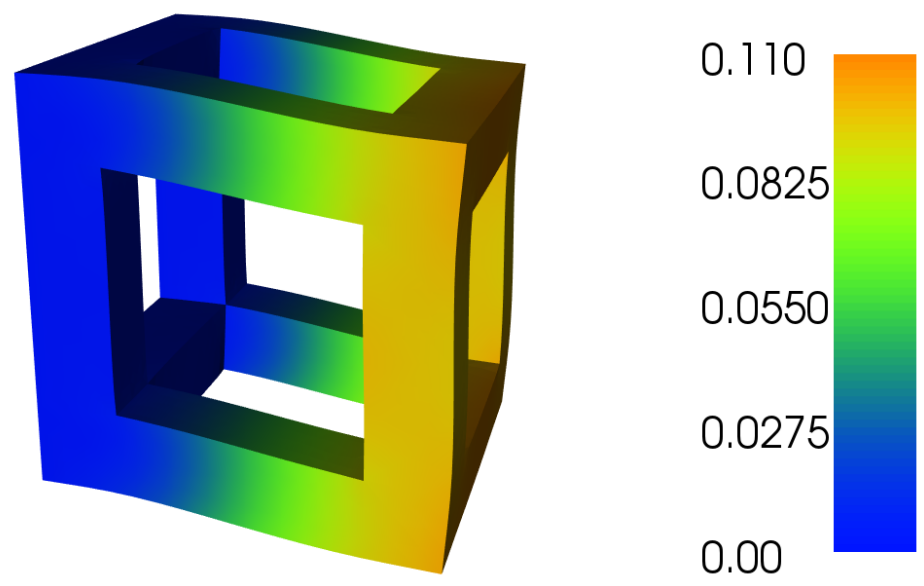

Figure 4: A 3-D domain $\Omega$ clamped at one end, deforming (units in $m$ ) under the influence of self weight and an external force applied at the opposite end 


\subsection{Acoustic Wave Equation}

As discussed in chapter 2, there are two popular approaches in the literature to model seismic waves propagation, i.e., the acoustic (scalar) wave equation, and the elastic (vector) wave equation. We begin by deriving the weak form of the linear scalar wave equation and implement it using FEniCS. The strong form (PDE) is given as [11]:

$$
\frac{\partial^{2}}{\partial t^{2}} u(x, t)=\nabla \cdot\left[c^{2}(x) \nabla u(x, t)\right]+f(x, t) \quad \text { on } \quad \Omega \times(0, T]
$$

To facilitate the time stepping scheme, we use an additional variable $p$ and convert

the equation into a first order system. The displacement and velocity at a point $x$ and time $t$ are denoted by $u(x, t)$ and $p(x, t)$.

$$
\begin{aligned}
& \frac{\partial u}{\partial t}=p \\
& \frac{\partial p}{\partial t}=\nabla \cdot\left(c^{2} \nabla u\right)+f
\end{aligned}
$$

with the boundary condition : $\quad u(x, t)=g(x, t) \quad$ on $\quad \partial \Omega \times(0, T]$,

$$
\begin{array}{ll}
\text { initial condition at } \mathrm{t}=0: & u(x, 0)=u_{0}(x) \text { in } \Omega, \\
\text { initial velocity at } \mathrm{t}=0: & p(x, 0)=u_{1}(x) \text { in } \Omega
\end{array}
$$

The following derivation closely follows from the concepts described in [107], [114], and [115]. For every solution $u(x, t)$ of this problem, the following should also be true:

$$
\int_{\Omega}\left(\frac{\partial}{\partial t} p(x, t)-c^{2}(x) \Delta u(x, t)\right) v(x) d x=\int_{\Omega} f(x, t) v(x) d x, \quad \forall v \in \hat{V}
$$


where $v$ is an arbitrary test function in the function space $\hat{V}$. Separate function spaces $\hat{V}$ and $V$ are considered for the test and trial function respectively.

$$
\begin{aligned}
& \hat{V}=\left[v \in H^{1}(\Omega): v=0 \quad \text { on } \quad \partial \Omega\right] \\
& V=\left[\begin{array}{ll}
v \in H^{1}(\Omega): v=u_{0} \quad \text { on } & \partial \Omega
\end{array}\right]
\end{aligned}
$$

where $H^{1}$ is a Hilbert-space containing function $v$ [107]. Applying integration by parts and Green's formula to Eq. 23 yields:

$$
\begin{gathered}
\int_{\Omega}\left(\frac{\partial}{\partial t} p(x, t)-\nabla \cdot\left[c^{2}(x) \nabla u(x, t)\right]\right) v(x) d x= \\
=\int_{\Omega} \frac{\partial}{\partial t} p(x, t) v(x) d x-\int_{\partial \Omega}\left[c^{2}(x) \partial_{n} u(x, t)\right] v(x) d s+\int_{\Omega}\left[c^{2}(x) \nabla u(x, t)\right] . \nabla v(x) d x
\end{gathered}
$$

where $n$ is the outward normal vector at the boundary. The test function $v$ is zero on the parts of the boundary where $u$ is known (Eq. 24). Hence, the right hand side of Eq. 26 becomes:

$$
=\int_{\Omega} \frac{\partial}{\partial t} p(x, t) v(x) d x+\int_{\Omega}\left[c^{2}(x) \nabla u(x, t)\right] \cdot \nabla v(x) d x
$$

The weak form of Eq. 22 is therefore given as: find $p \in V(0, T ; \Omega)$ such that

$$
\int_{\Omega} \frac{\partial p}{\partial t} v d x+\int_{\Omega}\left[c^{2}(x) \nabla u(x, t)\right] . \nabla v d x=f v d x \quad \forall v \in \hat{V}(\Omega), t \in(0, T]
$$

Substituting $\langle.,$.$\rangle for the inner product and a(u, v)=\int_{\Omega} \nabla u \cdot \nabla v d x$, we may write the weak form as

$$
\left\langle\frac{\partial p}{\partial t}, v\right\rangle+a(u, v)=\langle f, v\rangle, \quad \forall v \in \hat{V}(\Omega), u \in V(\Omega), t \in(0, T]
$$


Later on (after discretization), this can be expressed more conveniently in the bilinear form as $[107]$

$$
a(u, v)=L(v) \quad \forall v \in \hat{V}, u \in V
$$

The formulation in Eq. 28 should now be discretized in time and space to obtain the final variational form.

\subsubsection{Semidiscretization of Weak Form}

In FEM it is often useful to consider $u(x, t)$ as a function of $t$ using values in functions of $x$. To discretize the weak form of the wave equation, the first step is to discretize the spatial variable. To obtain a linear system of ODEs, we begin by approximating $u(x, t) \approx u_{h}(x, t)=\Sigma_{i} u_{i}(t) \phi_{i}(x)$, where $\phi_{i}(x)$ is a basis function for $V_{h}$. The basis functions $\phi_{i}(x)$ form a linear combination in space $V$, to represent the finite element function $u$. This is because for a semidiscrete approximation, we seek $u_{h}$ mapping $[0, T]$ into $V_{h} \in H^{1}(\Omega)$, i.e., find $u_{h}(t) \in V_{h}$ with boundary condition $u_{h}=g$ on $\partial \Omega$, satisfying

$$
\left\langle\frac{\partial p}{\partial t}, v\right\rangle+a(u, v)=\langle f, v\rangle, \quad \forall v \in \hat{V}_{h}, t \in[0, T]
$$

where the initial condition for $u$ is $u_{h}(0)=u_{h}^{0} \in V_{h}$. Similarly, $p(x, t) \approx p_{h}(x, t)=$ $\Sigma_{i} p_{i}(t) \phi_{i}(x)$ for shape function $\phi_{i}(x)$. Therefore, assuming $\phi_{j}$ equal to test function $v$, form 31 can be expressed as

$$
\sum_{i} \frac{\partial}{\partial t} p_{i}(t)\left\langle\phi_{i}, \phi_{j}\right\rangle+\sum_{j} u_{i}(t) a\left(\phi_{i}, \phi_{j}\right)=\left\langle f, \phi_{j}\right\rangle
$$

Introducing the stiffness matrix, mass matrix, and load vector as:

$$
M=m_{i j}=\left\langle\phi_{i}, \phi_{j}\right\rangle, \quad K=k_{i j}=a\left(\phi_{i}, \phi_{j}\right), \quad F=f_{j}(t)=\left\langle f, \phi_{j}\right\rangle
$$


the structure of the problem can now be captured as a regular second-order ODE in time:

$$
\begin{gathered}
\qquad M \ddot{u}(t)+K u(t)=F(t), \quad t \in[0, T] \\
\text { with initial conditions } u(0)=u_{0}, \quad p(0)=p_{0}
\end{gathered}
$$

which is similar to the equation of a mass-spring system and for a zero source term $(F(t)=0)$, it can also be written in block form as:

$$
\left(\begin{array}{cc}
I & 0 \\
0 & M
\end{array}\right)\left(\begin{array}{l}
\dot{u} \\
\dot{p}
\end{array}\right)=\left(\begin{array}{cc}
0 & I \\
-K & 0
\end{array}\right)\left(\begin{array}{l}
u \\
p
\end{array}\right)
$$

\subsubsection{Numerical Methods for Time Discretization}

In the semidiscrete form, time evolution was completely decoupled from the spatial operators and the problem was recast into a (continuous time system of) ODE(s). However, we now turn to fully discrete approximation by using a suitable time stepping scheme.

There are many explicit and implicit methods used in numerical analysis for obtaining numerical solutions of time-dependent PDEs, as is required in computer sim-

ulations of physical processes. Some popular schemes used in literature [116-118] for time discretization of the wave equation are:

1. Sympletic - Euler method

2. Crank Nicolson scheme

3. Newmark - Beta method

4. Leapfrog scheme 
Numerical stability of the time integration scheme is of utmost importance for simulation of any physical model. Implicit schemes are preferred owing to superior numerical stability for larger time steps, while the explicit schemes are popular due to ease of implementation, even though they require very small time steps. Vandekerckhove [119] and Tarplett [120] are excellent references to learn more about the various aspects of wave modelling using FEM (implemented using FEniCS in particular). A classical leapfrog time scheme was tested in [119] for application in non-destructive testing and evaluation, while an explicit formula was implemented in [120] for modelling linear seismic wave equation. In this study, we use the unconditionally stable and second order accurate, implicit Newmark - Beta scheme which allows for large time steps that prove convenient for solving problems like seismic wave propagation which may require the computational model to run for long time periods.

\section{Newmark Beta time discretization for Wave Equation}

The Newmark beta method [121] exploits Taylor expansion theorem and expresses the semidicretised wave equation in terms of displacement $u_{n}$, velocity $p_{n}$ and acceleration $a_{n}$ with respect to their first time derivative for each step as follows:

$$
\begin{aligned}
u_{n+1} & =u_{n}+\Delta t p_{n}+\frac{\Delta t^{2}}{2} a_{n+2 \beta} \\
p_{n+1} & =p_{n}+\Delta t a_{n+\gamma} \\
a_{n+2 \beta} & =(1-2 \beta) a_{n}+2 \beta a_{n+1} \\
a_{n+\gamma} & =(1-\gamma) a_{n}+\gamma a_{n+1}
\end{aligned}
$$

The two parameters $\beta$ and $\gamma$ determine the stability and accuracy characteristics of the algorithm. The choice of $\gamma=\frac{1}{2}$ and $\beta=\frac{1}{4}$ leads to an unconditionally stable time-integration operator of second order accuracy [122]. Using the time updating 
scheme above, the fully discretised form for Eq. 34 can be written as:

$$
\begin{gathered}
M a_{n+1}+K\left[u_{n}+\Delta t p_{n}+\frac{\Delta t^{2}}{2}\left((1-2 \beta) a_{n}+2 \beta a_{n+1}\right)\right]=f_{n+1} \\
\left(M+\beta(\Delta t)^{2} K\right) a_{n+1}=f_{n+1}-K\left[u_{n}+\Delta t p_{n}+\frac{\Delta t^{2}}{2}(1-2 \beta) a_{n}\right]
\end{gathered}
$$

\section{Courant-Friedrichs-Lewy condition}

The CFL condition needs to be levied for convergence while solving hyperbolic PDEs [123]. It establishes the following relationship

$$
c \Delta t \leq \Delta x
$$

where $c$ is the magnitude of velocity, $\Delta t$ is the time step, and $\Delta x$ is the mesh resolution. In simple words, this condition implies that the chosen time step cannot exceed the time required for a wave to travel across a single cell.

The acoustic waves shown in Fig. 5 and 6 were simulated using a finite element code created in python, based on FEniCS. The 2-D square domain of size $[1,1]$ has been discretised into triangular elements of second order with six nodes per element. The mesh resolution $\Delta h$ is 0.1 and the time step $\Delta t$ is 0.001 . A study conducted by Semblat [124] suggested the use of square-diamond patterned triangular elements for modelling wave propagation problems with higher accuracy. Also, triangular elements make it easier to mesh over complicated geometry and can easily adapt to meshes that require refinement at certain nodes. As such, all 2-D domains considered throughout this study have been triangulated. A Gaussian Pulse was used as a source at the centre of mesh and the Newmark Beta scheme was utilised for time stepping. Visualisation was done using ParaView [125]. 

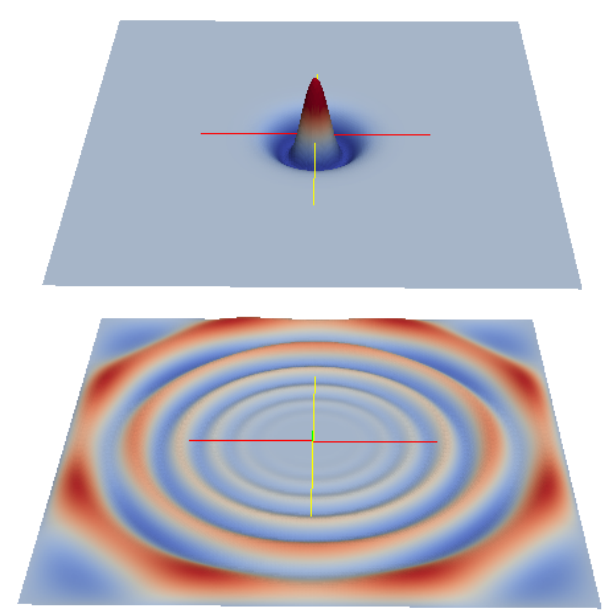
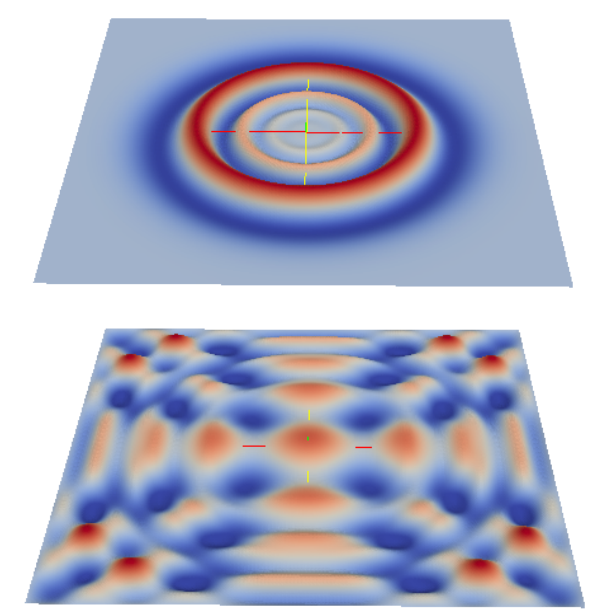

Figure 5: Magnitude of acoustic waves in a homogeneous isotropic elastic medium

Seismic anisotropy is the directional dependence of seismic velocities. It can be observed that the wave propagation is completely symmetric in Fig. 5 for the isotropic model, while the shape of unsymmetrical wave fronts at different spatial locations from the source (in Fig. 6) can be attributed to the anisotropic effects.

\subsection{Elastic Wave Equation}

We now consider the case of linear elastic wave propagation. As discussed previously in chapter 2, the elastic (vector) wave or the linear elastodynamics equation is the most accurate option to model seismic wave propagation. It has the form,

$$
\rho \ddot{u}_{i}=f_{i}+\sigma_{i j, j}
$$

with the boundary bondition : $\quad u(x, t)=h(x, t) \quad$ on $\quad \partial \Omega \times(0, T]$,

$$
\text { initial condition at } \mathrm{t}=0: \quad u(x, 0)=u_{0}(x) \text { in } \Omega
$$

$$
\text { initial velocity at } \mathrm{t}=0: \quad \dot{u}(x, 0)=v_{0}(x) \text { in } \Omega
$$



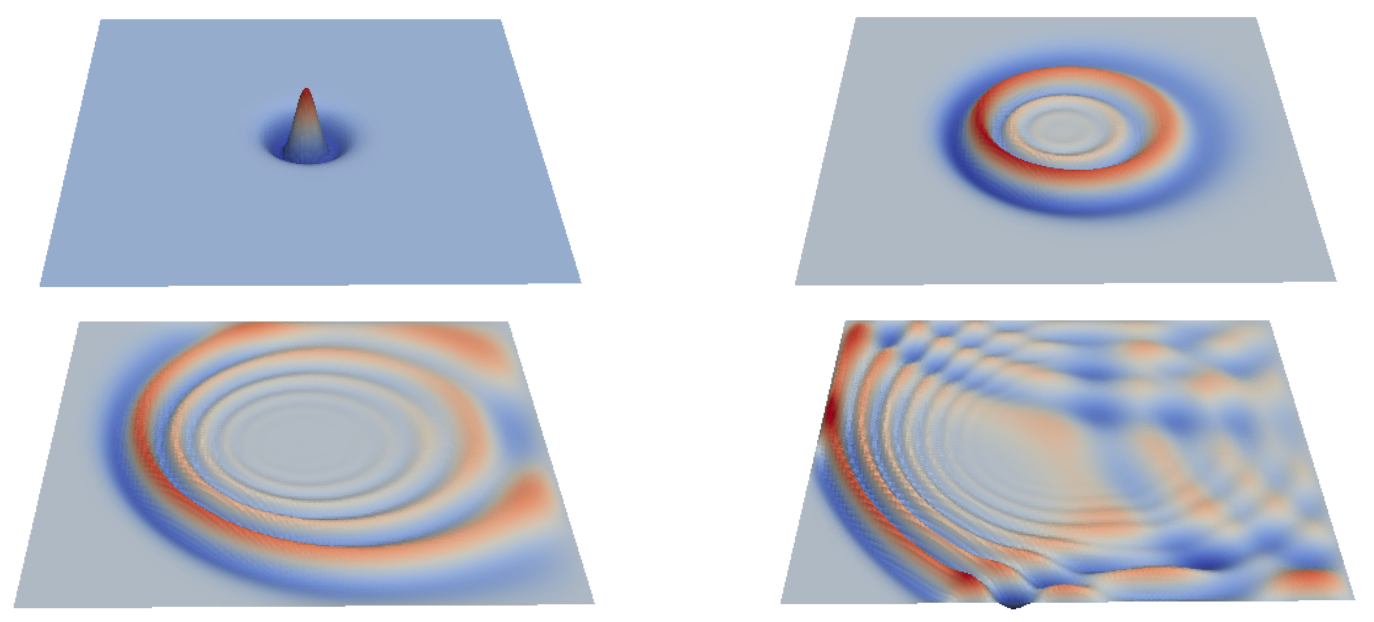

Figure 6: Magnitude of acoustic waves in a homogeneous anisotropic medium where the wave speed $c$ varies spatially as $2 \sin (x)+y$

where $\ddot{u}, \dot{u}$, and $u$ represent the particle acceleration, velocity and displacement respectively; $f$ is the body force, and $\rho$ is the density of the medium. In order to create the finite element model, we need to derive the weak form of the Eq. 39 by multiplying it with a weight function $v$ and integrating over the domain $\Omega$ :

$$
\int_{\Omega} \rho \ddot{u} \cdot v d x-\int_{\Omega}(\nabla \cdot \sigma) \cdot v d x-\int_{\partial \Omega} h \cdot v d s-\int_{\Omega} f \cdot v d x=0
$$

Applying integration by parts along with the divergence theorem, we get the following weak form [81, 107]:

$$
\int_{\Omega} \rho \ddot{u} \cdot v d x+\int_{\Omega} \sigma: \nabla v d x-\int_{\partial \Omega} h \cdot v d s-\int_{\Omega} f \cdot v d x=0
$$

Here $\partial \Omega$ is the boundary of domain $\Omega$, and $h$ is the prescribed traction on part of the boundary. Based on the variational form noted above, we develop a a finite element code based on python and FEniCS for solving the problem of elastic wave propagation. The code is validated by comparing the results with the 1-D elastic wave propagation example from PLAXIS manual [126], as also done in [127]. A 1-D 
wave simulation is performed on a $10 \mathrm{~m}$ long bar using the following soil properties as given in [126]:

$$
\rho=19.62 k N / m^{3}, \quad E=18000 k N / m^{2}, \quad \nu=0.2
$$

where, $E=$ Young's modulus and $\nu=$ Poisson's ratio. The time step and the mesh resolution were chosen in accordance with the CFL condition. The $\mathrm{P}$ wave velocity in a 1-D medium can be given as [11]:

$$
V_{p}=\sqrt{\frac{E(1-\nu)}{\rho(1+\nu)(1-2 \nu)}}
$$

The value of $V_{p}$ for the given material properties equals $100 \mathrm{~m} / \mathrm{s}$ and therefore, the wave generated at the left end of the bar should reach the centre of the mesh in exactly $0.05 \mathrm{~s}$. The source has been provided in the form a time dependent Dirichlet BC (sine wave) at the left end of the bar. The simulation has been shown in Fig. 7.

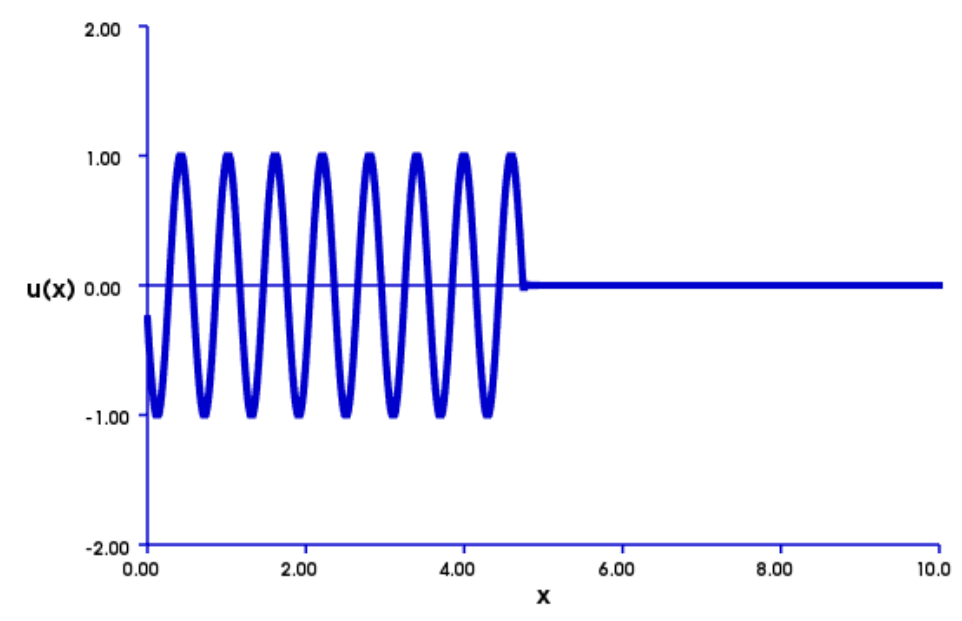

Figure 7: 1-D wave at time $0.05 s$ propagating on a $10 \mathrm{~m}$ long bar

We will now extend our elastic model to a visco-elastic model [107] by modifying the 
stress tensor given in Eq. 18 as

$$
\sigma(u))=2 \mu \epsilon(u)+(\lambda \operatorname{tr}(\epsilon(u))+\eta \operatorname{tr}(\dot{\epsilon}(u))) I
$$

where $\eta \geq 0$ is a constant viscosity parameter. A 2-D elastic wave simulation in a homogeneous isotropic domain has been shown in Fig. 8 for a large valley which is $1.2 \mathrm{~km}$ long and $600 \mathrm{~m}$ deep at the centre. The $2-\mathrm{D}$ domain has been discretised into triangular elements of first order with three nodes per element. The values used for physical parameters of soil are:

$$
G=38.5 M P a, \quad \nu=0.3, \quad \rho=1700 \mathrm{~kg} / \mathrm{m}^{3}, \quad \eta=5 \%
$$

where $G$ represents the shear modulus. These values correspond to a shear wave velocity $\left(V_{s}=\sqrt{G / \rho}\right)$ of $150 \mathrm{~m} / \mathrm{s}$. A Gaussian pulse in space $f=\left(0, f_{x}\right)$ is applied to the centre of the mesh, where

$$
f_{x}=\beta \exp \left(-\frac{\left(x-x_{0}\right)^{2}+\left(y-y_{0}\right)^{2}+\left(z-z_{0}\right)^{2}}{\alpha}\right)
$$

where $\beta$ is the amplitude of the pulse and $\alpha$ determines the width of the pulse. In Fig. 8 , the valley has a free surface at the ground level and reflecting condition $(u=0)$ on the rest of the boundary. The boundary condition $u=0$ makes $u$ change sign at the boundary and reflect back. It can be observed how the Gaussian pulse at the centre propagates symmetrically in all directions and reflects back when it hits the boundaries. 

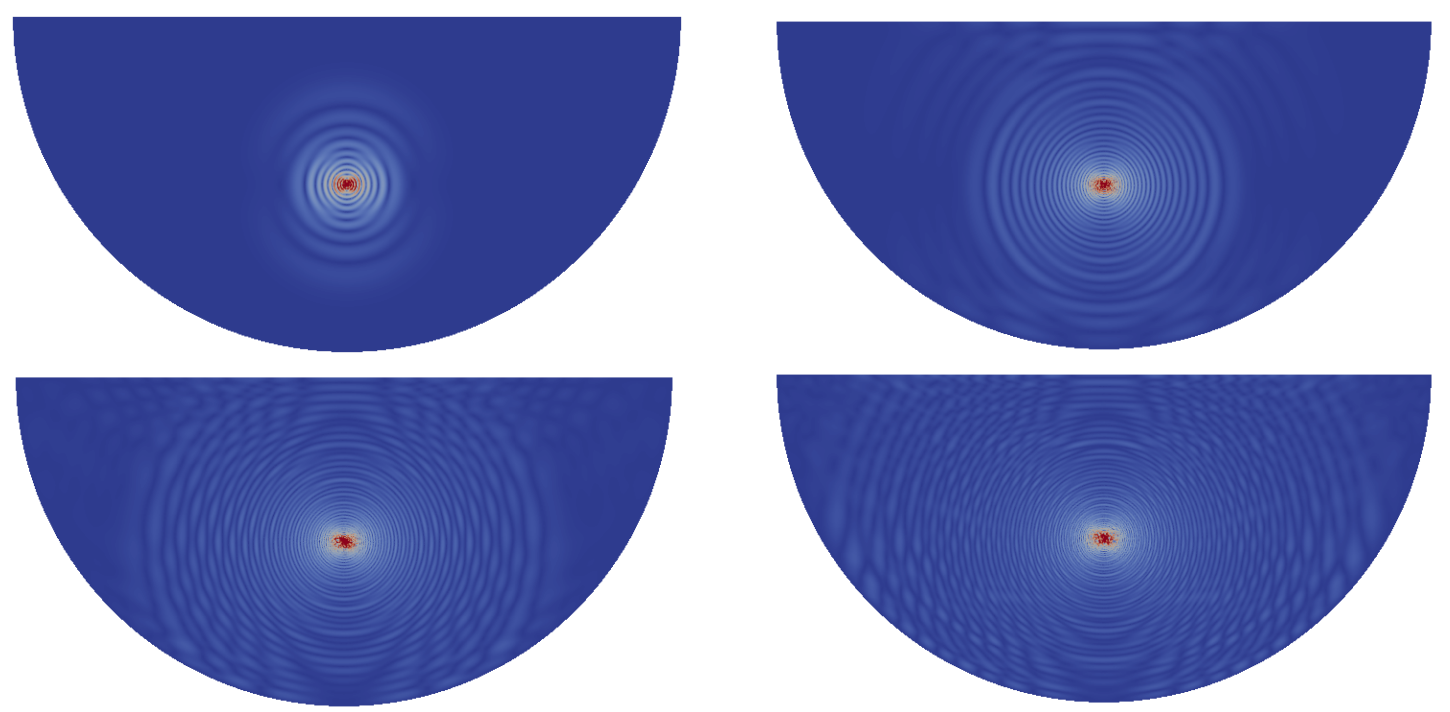

Figure 8: Magnitude of elastic waves in a homogeneous isotropic medium $(1.2 \mathrm{~km}$ wide and $0.6 \mathrm{~km}$ deep), at different time frames

\subsection{Elasticity to Classical Plasticity}

The objective of current and the forthcoming sections is to present the concepts of plasticity in a theoretical and computational framework, in order to provide background for implementation discussed later in this chapter. The concepts described henceforth are based on $[4,128-132]$ and the readers are directed to these references if they wish to explore these concepts in detail. The transition from linear elasticity to elasto-plasticity requires a thorough understanding of the basics of non-linear elasticity and plasticity. In linear elastostatic analysis we assume that the material will experience infinitesimal deformation which can be satisfactorily represented by a linear constitutive equation, i.e., the Hooke's law is assumed valid. However, when the loads are high and the material under consideration is undergoing major displacement, different types of non-linearities may be encountered. The major difference between linear and non-linear elasticity is the non-linear stress-strain relationship observed in the elastic regime, for case of the latter. 
This behaviour may be attributed to the material properties (material nonlinearity), and considerable changes in the geometry during load application (geometric non-linearity). This means that an object experiencing both, high stress and deformation, will neither follow the linear constitutive equation (due to material non-linearity), nor will it show a linear stress-strain trend (owing to geometric nonlinearity). Materials such as steel, soil and concrete, when subjected to high compression or tension will display material non-linearity through a non-linear stress-strain curve even at relatively small displacements, while geometric non-linearity will kick in mostly under large deformation.

The classical theory of plasticity can be considered an extension of the theory of elasticity to account for material non-linearity and the presence of irrecoverable strains. A plastic solid will show a non-unique stress-strain relationship while experiencing a loading sequence evolving in time, and undergo a permanent deformation on load removal. This constitutes the fundamental difference between non-linear elasticity and plasticity. Therefore, if a material depicts a non-linear stress-strain relationship upon loading, it cannot be concluded that the behaviour is non-linear elastic or plastic. Only during unloading, it becomes possible to identify the difference, because an elastic material shall follow the same path while a plastic material will show a history-dependent different path and an irreversible plastic strain $\epsilon^{p}$ shall remain [3]. This phenomena has been shown in Fig. 9, where the yield stress is represented by variable $\sigma_{y}$. It is worth noting here that in the case of non-linear elasticity, the unloading curve may not necessarily coincide with the loading curve, thereby exhibiting hysteresis effect. However, the recovery of the material will be complete and no plastic strain shall be observed. 

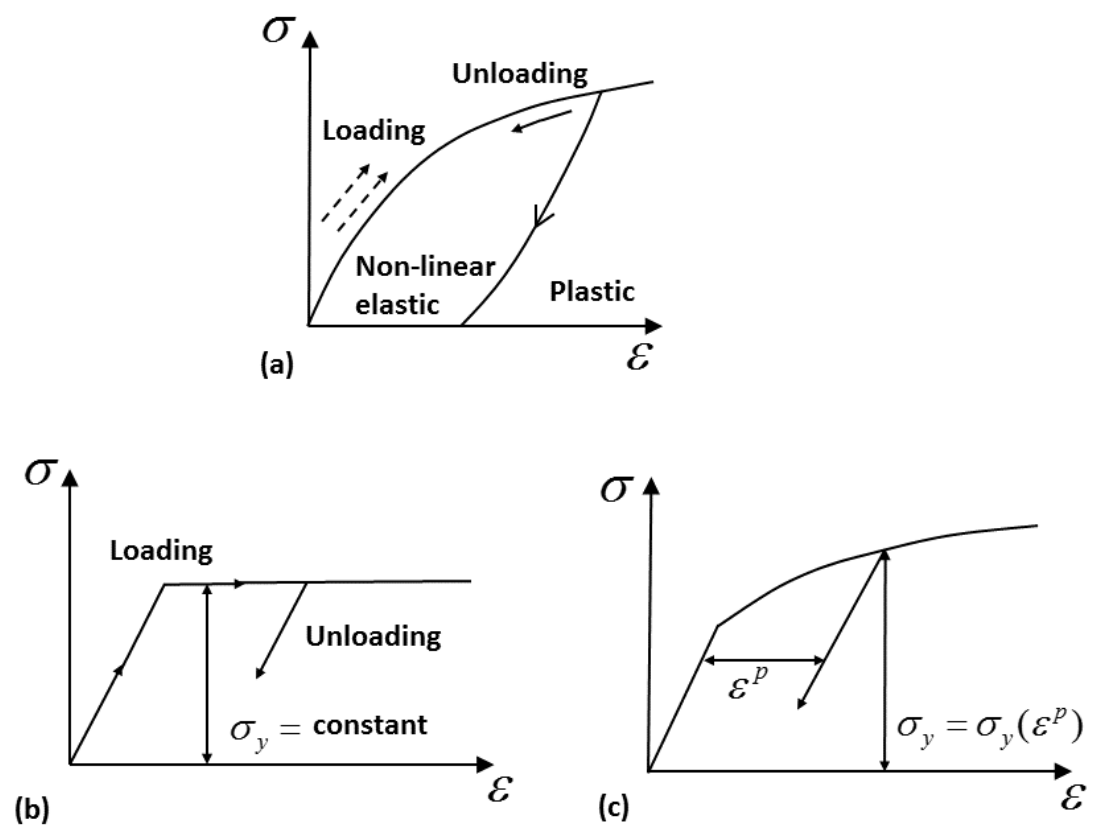

Figure 9: (a) Non-linear elastic and elasto-plastic; (b) Linear elastic-perfectly plastic; (c) elasto-plastic with strain hardening [3]

\subsection{Theory of Plasticity}

The theory of plasticity builds upon the standard elements of elasticity derived from continuum mechanics, and attempts to explain the response of solids that have experienced loading outside their elastic regime.

\subsubsection{Rate dependence in Plasticity}

Various plastic constitutive laws have been developed in literature and each is suitable for a specific set of applications. Two broad classes exist:

1. Rate independent plasticity: Once the material experiences a certain level of stress (yield stress $=\sigma_{y}$ ), an irreversible straining occurs in it. Such plastic strains are assumed to develop instantaneously and are not dependent on time, 
i.e., independent of the strain rate. This characterizes rate-independent plasticity.

2. Rate dependent plasticity: Also known as viscoplasticity, the rate-dependent plasticity assumes that the plastic deformation, i.e., flow rule of materials is a function of the rate at which loads are applied (strain rate). It is different from rate-independent models because it is also capable of modelling time dependent creep flow, in addition to the plastic deformation. It is mostly used to model high temperature creep and deformation at high strain rates, where flow strength is sensitive to deformation rate.

- For geotechnical applications based on elasto-plasticity, soils are mostly modelled as continuum rate-independent materials. This approach has a relatively straight forward formulation compared to the rate-dependent method and has been implemented in many studies concerning ground motion analysis $[16,25,28]$. However, as reported in [133], it may prove to be slightly computationally intensive for large-scale earthquake ground motion simulations due to the iterative procedure involved in prediction of plastic strain $\epsilon^{p}$, and therefore in such cases, a rate-dependent (Elasto-visco-plasctic) formulation may be more suitable (eg., [39, 40, 134]). We shall discuss computational plasticity in detail later. For our model, we assume a rate-independent plasticity and focus on elasto-plastic problem which can also be referred to as a limiting case of viscoplasticity. In fact, the rate-independent formulation is considered to be a special case of rate-dependent theory $[135,136]$. 


\subsubsection{Understanding Plasticity Models}

When the applied loading reaches the yield stress of a material, the total strain

$(\epsilon)$ that occurs has a plastic $\left(\epsilon^{p}\right)$ and an elastic part $\left(\epsilon^{e}\right)$, i.e., $\epsilon=\epsilon^{p}+\epsilon^{e}$. This distinction forms the basis of elasto-plasticity because separate constitutive models are used for elastic and plastic part. We are already aware of the Hooke's law which is implemented for elasticity. In summary, there are three key ingredients that form the essential characteristics of any plastic constitutive model:

- The yield criterion defining the limit at which the material becomes plastic.

- An accompanying flow rule that determines the direction of the plastic straining and increment in plastic strain from the increment in load.

- The hardening function that controls the change in yield function due to incurring plastic strain.

A detailed description will be provided in subsequent sections. However, before we proceed, it is important to understand the concept of stress invariants and plane strain.

\section{Concept of Stress Invariants}

Following up on the linear elastostatics section 3.2.1, the second order Cauchy stress tensor, which defines the state of stress at any point, is given by:

$$
\boldsymbol{\sigma}=\sigma_{i j}=\left[\begin{array}{ccc}
\sigma_{11} & \sigma_{12} & \sigma_{13} \\
\sigma_{21} & \sigma_{22} & \sigma_{23} \\
\sigma_{31} & \sigma_{32} & \sigma_{33}
\end{array}\right] \equiv\left[\begin{array}{ccc}
\sigma_{x x} & \sigma_{x y} & \sigma_{x z} \\
\sigma_{y x} & \sigma_{y y} & \sigma_{y z} \\
\sigma_{z x} & \sigma_{z y} & \sigma_{z z}
\end{array}\right] \equiv\left[\begin{array}{ccc}
\sigma_{x} & \tau_{x y} & \tau_{x z} \\
\tau_{y x} & \sigma_{y} & \tau_{y z} \\
\tau_{z x} & \tau_{z y} & \sigma_{z}
\end{array}\right]
$$


Here, $\sigma_{x}, \sigma_{y}$ and $\sigma_{z}$ represent the normal stresses and the rest are shear stresses. Three principal planes exist at each point in a stressed body. When there are no shear stresses involved, the three stresses normal to these principal planes are called principal stresses. On a 2-D element, there exists a 'major' principal stress and a 'minor' principal stress, while on a 3-D element there are three principal stresses, i.e., 'minor', 'intermediate', and 'major' principal stress.

The magnitude of the components in $\sigma_{i j}$ depends upon the orientation of the coordinate system at the point under consideration. For example, when an object is subjected to a uni-axial (1-D) tension or compression in $x$-direction, it is very important that its alignment in the coordinate system is such that all the other stress components except for $\sigma_{x}$ remain zero. However, as the object deforms under stress, a rotation of the coordinate system is likely. In a plasticity model, where the yield criteria needs to be fixed to allow for plastic flow, it must be ensured that the condition at which the material yields is not influenced by such rotation.

This gives rise to the need of certain invariant quantities related with every tensor, which remain uninfluenced by the rotation of coordinate system. A set of such invariants are the principal stresses (eigenvalues of the stress tensor), which act in the same direction with same magnitude for a given stress state, irrespective of the chosen orientation of coordinate axes. Let's consider $\sigma_{1}, \sigma_{2}$ and $\sigma_{3}$ to be the principal stresses.

The Cauchy stress tensor can be divided into two separate components, namely the mean hydrostatic or volumetric stress tensor, $\pi \delta_{i j}$, which is responsible for change in volume of the body (dilation), and the deviatoric component called the deviator stress tensor, $s_{i j}$, which actually causes distortion of the body.

$$
\sigma_{i j}=s_{i j}+\pi \delta_{i j}
$$


Here, the isotropic component, i.e., the mean hydrostatic stress is given by [131]:

$$
\pi=\frac{\sigma_{k k}}{3}=\frac{\sigma_{11}+\sigma_{22}+\sigma_{33}}{3}=\frac{1}{3} I_{1}
$$

where $I_{1}, I_{2}$, and $I_{3}$ constitute another, more convenient, set of invariants of the stress tensor, but we only use $I_{1}$ in the concepts that follow. The Cauchy stress tensor can be decomposed into two previously mentioned components in Voigt notation as:

$$
\begin{aligned}
\sigma_{i j} & =s_{i j}+\frac{\sigma_{k k}}{3} \delta_{i j}, \\
{\left[\begin{array}{ccc}
\sigma_{11} & \sigma_{12} & \sigma_{13} \\
\sigma_{21} & \sigma_{22} & \sigma_{23} \\
\sigma_{31} & \sigma_{32} & \sigma_{33}
\end{array}\right] } & =\left[\begin{array}{lll}
s_{11} & s_{12} & s_{13} \\
s_{21} & s_{22} & s_{23} \\
s_{31} & s_{32} & s_{33}
\end{array}\right]+\left[\begin{array}{ccc}
\pi & 0 & 0 \\
0 & \pi & 0 \\
0 & 0 & \pi
\end{array}\right] \\
s_{i j} & =\left[\begin{array}{lll}
\sigma_{11}-\pi & \sigma_{12} & \\
\sigma_{21} & \sigma_{22}-\pi & \sigma_{13} \\
\sigma_{31} & \sigma_{32} & \sigma_{33}-\pi
\end{array}\right] .
\end{aligned}
$$

Just like the invariants of the Cauchy stress tensor, it's component, the deviatoric stress tensor, $s_{i j}$, also has invariants $J_{1}, J_{2}$ and $J_{3}$. It is reported in literature that $I_{1}$ and $J_{2}$ are especially useful for plasticity models $[137,138]$. The second invariant of $s_{i j}$, i.e., $J_{2}$ is given as [131]:

$$
\begin{aligned}
J_{2} & =\frac{1}{2} s_{i j} s_{i j} \\
& =\frac{1}{6}\left[\left(\sigma_{11}-\sigma_{22}\right)^{2}+\left(\sigma_{22}-\sigma_{33}\right)^{2}+\left(\sigma_{33}-\sigma_{11}\right)^{2}\right]+\sigma_{12}^{2}+\sigma_{23}^{2}+\sigma_{31}^{2} \\
& =\frac{1}{6}\left[\left(\sigma_{1}-\sigma_{2}\right)^{2}+\left(\sigma_{2}-\sigma_{3}\right)^{2}+\left(\sigma_{3}-\sigma_{1}\right)^{2}\right]
\end{aligned}
$$




$$
=\frac{1}{3} I_{1}^{2}-I_{2}
$$

\section{Plane Strain}

Sometimes, while dealing with elasticity, especially in geotechnical analysis, problems that have one very large spatial dimension compared to the others are often encountered. For example, say the dimension in z-direction is very large with respect to the dimensions of the structure in $x$ and $y$-coordinate axes. Such problems may be treated satisfactorily by a cross-sectional, two-dimensional representation, by assuming that the primary field variables in the long direction are zero. This is known as plane strain analysis. A typical representation has been shown in Fig. 10. Under such an assumption, the shear strain $\gamma_{x z}$ and $\gamma_{y z}$, and the normal strain to the $x-y$ plane, i.e., $\epsilon_{z}$, are considered zero. The strains in vector form will therefore look like:

$$
\epsilon=\left[\begin{array}{c} 
\\
\epsilon_{x} \\
\epsilon_{y} \\
\epsilon_{x y}
\end{array}\right]
$$

For the 2-D analysis performed in this study, plane strain condition is assumed and all the approximation is done through conversion of the the 3-D elasto-plastic equations.

\section{Yield Function}

The yield function forms a surface in three dimensional principle stress space to determine the stress level at which yielding is initiated, and has the units of stress. In a $1 \mathrm{D}$ case, it is convenient to define this limit by a single uni-axial yield stress 


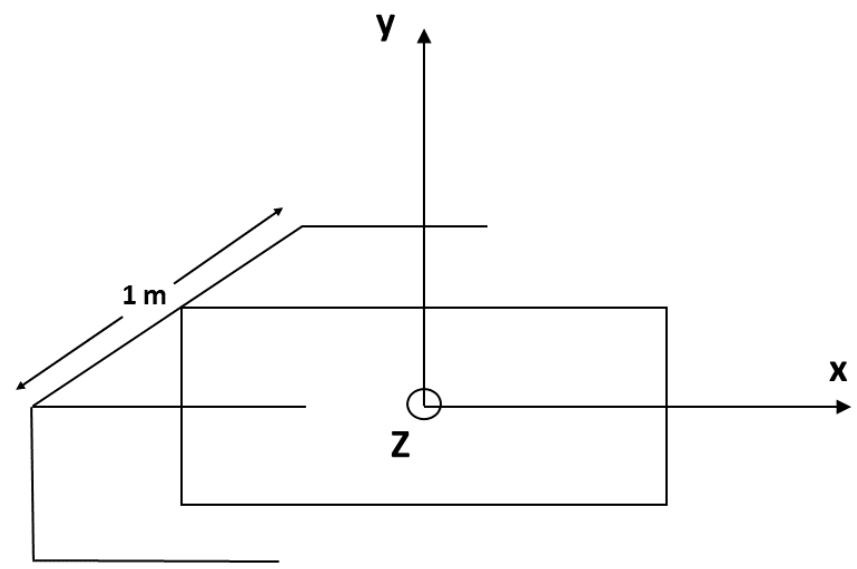

Figure 10: A plane strain element in the $x-y$ plane

(instead of 9 components of Cauchy stress tensor), but for higher dimensions implying multiple stress components, a function is needed to define the onset of plastic flow. This function is dependent on invariants of the principal or deviatoric part of the stress tensor. The stress states lying on or above the yield surface $(f \geq 0)$ are plastic while those lying within the yield surface $(f<0)$ are considered elastic. The scalar yield function for a perfectly plastic case is therefore, defined as:

$$
f(\boldsymbol{\sigma})=0
$$

A typical representation of a 2-D yield surface has been shown in Fig. 11. However, when hardening (or softening) of the material takes place with the loading cycle, the response is not perfectly plastic any more and the yield surface will evolve (change) with strain. To model this hardening, classical plasticity introduces a strain-like variable, the equivalent plastic strain $\xi_{p}$, a constant hardening parameter $H$, and the scalar internal material variable $\kappa$. The yield function for plasticity with hardening therefore becomes:

$$
f(\boldsymbol{\sigma}, \boldsymbol{\kappa})=0
$$


An interesting fact here is that, $(f>0)$ never actually occurs. This is implied by the

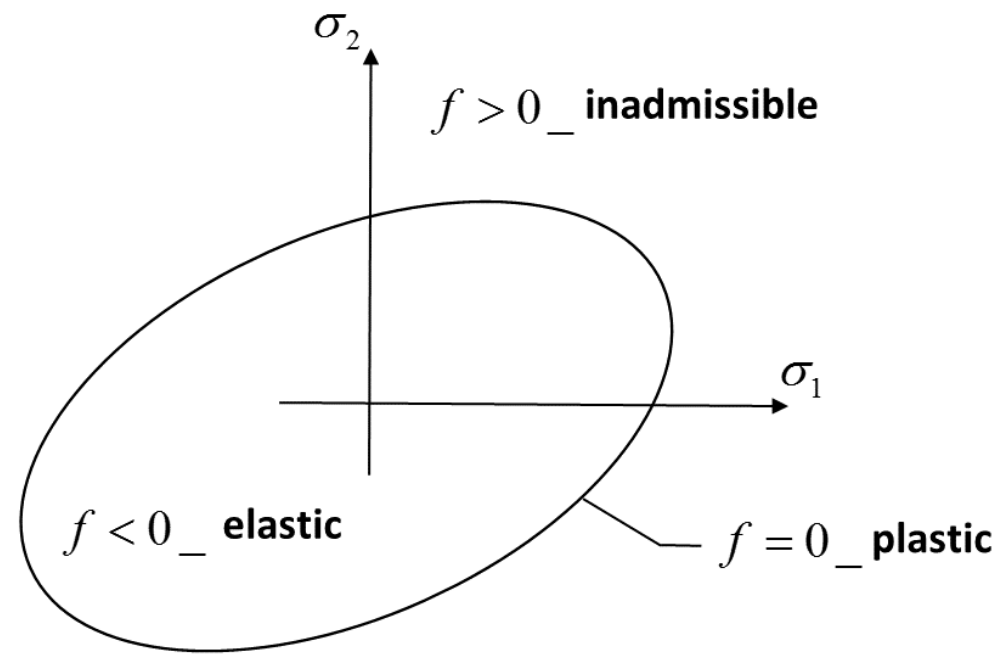

Figure 11: Yield surface in 2-D stress space [4]

Kuhn-Tucker consistency conditions for plasticity. When the plastic flow begins, i.e., $(f=0)$, and $\left(\sigma_{e q}=\sigma_{y}\right)$, one would assume that with increasing load, eventually $f$ and $\left(\sigma_{e q}-\sigma_{y}\right)$ would become greater than zero. However, the stresses must remain on the yield surface and therefore the stress states lying outside it are inadmissible, and must be redistributed between the different stress components (through an iterative procedure).

When the case is not perfectly plastic and some kind of hardening exists, then the yield surface keeps evolving with the load increment and the relevant hardening law, but the stress state still remains on the boundary defined by $(f=0)$.

\section{Flow Rule and The Plastic Potential}

As stated in the beginning of this section, the changes in strain (for elasto-plasticity) are assumed to consist of elastic and plastic components.

$$
d \epsilon=d \epsilon^{p}+d \epsilon^{e}
$$


Provided an elastic constitutive matrix $C$, the elastic strain can be related with stress through the following equation:

$$
d \sigma=C d \epsilon^{e}=C\left(d \epsilon-d \epsilon^{p}\right)
$$

However, as noted earlier, the plastic part, just like the elastic part, employs its own constitutive stress-strain laws. In an uni-axial case, the plastic flow follows the direction of stress. However, in higher dimensions, when there are multiple stress components, the following flow rule specifies the direction of plastic straining at every stress state.

$$
d \epsilon^{p}=d \Lambda\left(\frac{\partial g(\boldsymbol{\sigma})}{\partial \boldsymbol{\sigma}}\right)
$$

where $d \Lambda$ is a positive scalar quantity called plastic multiplier, which is dependent on the flow conditions and is not a property of the material; and $g(\boldsymbol{\sigma})$ is called the plastic potential function. The derivative of $g$ with respect to the corresponding stress, which also represents the normal vector to the plastic potential surface, specifies the direction of the plastic strain increment, while $d \Lambda$ sets its magnitude.

\section{Associated and Non-Associated plasticity}

Just like the yield function $f$, the plastic potential function, $g$, is also determined experimentally. As pointed out earlier, this is a cumbersome task for higher dimensions, where the testing is not uni-axial. Therefore, it is a reasonable assumption to equate the plastic potential surface with the yield surface, i.e.,

$$
\frac{\partial g(\boldsymbol{\sigma})}{\partial \boldsymbol{\sigma}}=\frac{\partial f(\boldsymbol{\sigma})}{\partial \boldsymbol{\sigma}}
$$


This approximation is known as the associated flow rule because the flow rule is associated with a particular yield criterion, unlike a non-associated flow rule, which has a separate yield and plastic potential function. An associated flow rule implies that the direction of plastic flow occurs in a direction normal to the yield surface. We consider the associated flow rule for our model. A detailed discussion on the selection of the same is postponed to section 3.6.2.

\section{Hardening Rules}

Consider a solid undergoing plastic deformation, then unloading, and then re-loading to induce further plastic flow. In the second cycle, its resistance to plastic flow, i.e., its yield point or elastic limit will have increased. This is known as 'strain hardening'. The hardening rule controls the evolution of yield surface (size,center,shape) after the yield stress is exceeded. The hardening rules which govern the change of the yield surface can be generally classified as isotropic and kinematic hardening.

Isotropic Hardening [139] implies that the yield surface evolves uniformly in all directions with plastic flow, while kinematic hardening [140] does not cause a change in the size of the yield surface, but translates it in the direction of yielding. This phenomena is shown in Fig. 12. The scalar internal variable, $\kappa$, introduced in (Yield Surface) section 3.6.2, characterizes the the condition, i.e., the state of elasto-plastic material. Actually, there exist multiple internal variables $\kappa_{1}, \kappa_{2} \ldots \kappa_{n}$, which represent the current state of the material by memorizing the plastic loading history. Also known as the hidden state variables, they cannot be directly observed or measured, and hold no value before any plasticity is initiated. We assume that only one variable $\kappa$ controls the change of size of the yield surface and therefore, ignore the rest. The linear isotropic hardening parameter, $H$, which is also known as the plastic modulus, 


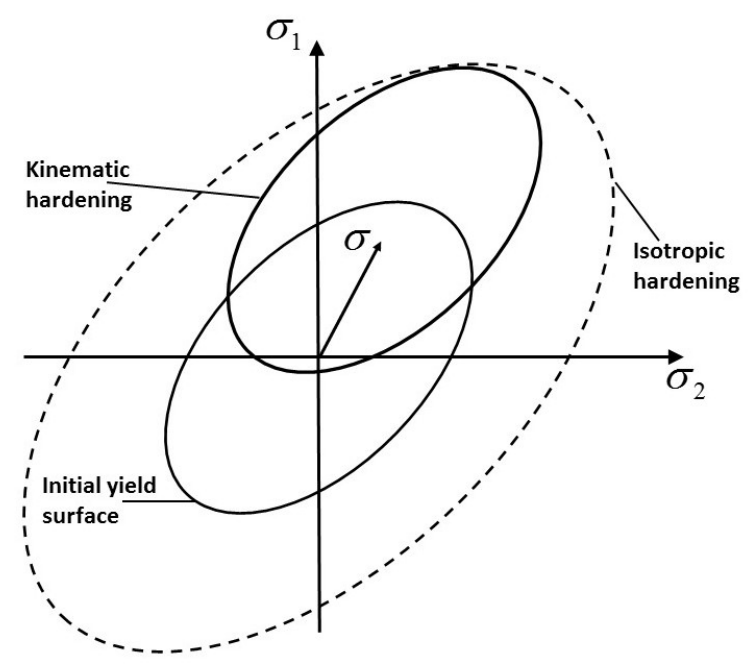

Figure 12: Evolution of isotropic and kinematic hardening with loading [5]

is given by

$$
H=\frac{d \sigma_{y}}{d \epsilon^{p}}
$$

for an uni-axial case. However, an equivalent plastic strain $\xi_{p}$ should be used for a multi-dimensional problem. For an associated flow rule, we have

$$
\begin{gathered}
d \xi_{p}=d \Lambda=d \kappa \\
H=\frac{d \sigma_{y}}{d \xi_{p}}=\frac{d \sigma_{y}}{d \Lambda}=\frac{d \sigma_{y}}{d \kappa}
\end{gathered}
$$

This shall be followed up in the subsequent section. To transition from isotropic to kinematic hardening we require another new variable known as the back stress, $\beta_{i j}$. However, kinematic hardening is beyond the scope of this thesis. Detailed explanation regarding all the concepts discussed previously, and in the following sections can found in popular references like $[4,138,141,142]$. The author also acknowledges the fact that isotropic hardening does not account for Bauschinger effect [143] and is therefore considered more suitable for monotonic loading. Kinematic hardening is more capable of modelling cyclic behaviour and Bauschinger effect. However, it 
results in a more complicated model and in such complex models, "parameters should be chosen such that they are closely related to the rheology that describes the material properties at various strain levels" [25]. Sometimes such models employ physical variables that cannot be evaluated by experimentation. This causes major difficulty in identification of parameters along with the required soil properties. In order to bypass these difficulties, we consider only isotropic hardening for our model.

\section{$J_{2}$ Plasticity}

In an elastic body experiencing loading in more than one dimensions, a complex three-dimensional state of stress is developed (i.e., the direction and magnitude of stresses in the body changes from point to point). The von Mises criterion defines the formula for evaluating whether the stress combination at a particular point will lead to failure. The criterion is built upon the discovery that, even though none of the principal stresses exceed the yield stress of the material, a combination of these stresses may result in yielding. It combines these three principal stresses into an equivalent stress, $\sigma_{e q}$ which is then compared to the yield stress of the material $\sigma_{y}$. It is also known as $J_{2}$ flow theory because the yield function can be expressed in terms of the second invariant of the deviatoric stress tensor. As mentioned earlier, the deviatoric stress is responsible only for distortion and not dilation. This yield criterion is commonly used in plasticity models for a wide range of materials, and can be expressed in conjunction with Eq. 57 as [131]

$$
\begin{aligned}
f(\boldsymbol{\sigma}) & =f\left(J_{2}\right) \\
\text { or, } \quad f: & =\sqrt{J_{2}}-k \leq 0
\end{aligned}
$$

where $k$ is the yield stress of the material in pure shear stress state. In such a condition, the material yields when the shear stress reaches $(1 / \sqrt{3})$ of $\sigma_{y}$, i.e., $k=$ 
$\sigma_{y} / \sqrt{3}$. Therefore, for perfect plasticity, i.e., no hardening, we have the following yield criterion $[130]$

$$
\begin{aligned}
f: & =\sqrt{3 J_{2}}-\sigma_{y} \leq 0 \\
\text { or, } \quad f: & =\|s\|-\sqrt{\frac{2}{3}} \sigma_{y} \leq 0
\end{aligned}
$$

where $s=s_{i j}$ is the deviatoric part of the stress tensor and $\|s\|$ implies the norm of the element of a normed vector space. The expression for von Mises equivalent stress is given as [132]:

$$
\sigma_{e q}=\sqrt{3 J_{2}}=\sqrt{\frac{3}{2} s_{i j} s_{i j}}
$$

We may accomplish linear isotropic hardening by writing [107, 130],

$$
\begin{aligned}
f(\boldsymbol{\sigma}, \kappa) & =\sqrt{3 J_{2}}-\sigma_{y}(\kappa)=0 \\
\sigma_{y}(\kappa) & =\sigma_{y 0}+H \kappa
\end{aligned}
$$

where $\sigma_{y 0}$ is the initial yield stress, $H_{\kappa}$ is the hardening parameter as defined previously, and $\dot{\kappa}=\dot{\Lambda}=\dot{\xi}_{p}$, is true for an associated flow (Eq. 63). Also, a suitable expression for equivalent plastic strain rate is given by [107, 132]

$$
\dot{\xi}_{p}=\sqrt{\frac{2}{3} \dot{\epsilon}_{i j}^{p} \dot{\epsilon}_{i j}^{p}}=\dot{\kappa}
$$

The factor of $2 / 3$ is introduced so that in an uni-axial tensile test we have $\xi_{p}=\epsilon_{11}$. The von Mises model is a good first approximation for metals, polymers, and saturated geological materials. The criterion is isotropic and independent of hydrostatic pressure, which makes it more suitable for incompressible materials which are less sensitive to the influence of hydrostatic pressure, and do not exhibit much plastic dilation. As shown in Fig. 13, the yield surface is oriented along the hydrostatic 
axis $\sigma_{1}=\sigma_{2}=\sigma_{3}$ in a 3 -D principal stress space. This axis represents a hydrostatic stress state and is called the space diagonal. Fig. 14 depicts the concept of isotropic hardening by showing that the maximum stress in compression becomes the yield stress in tension.

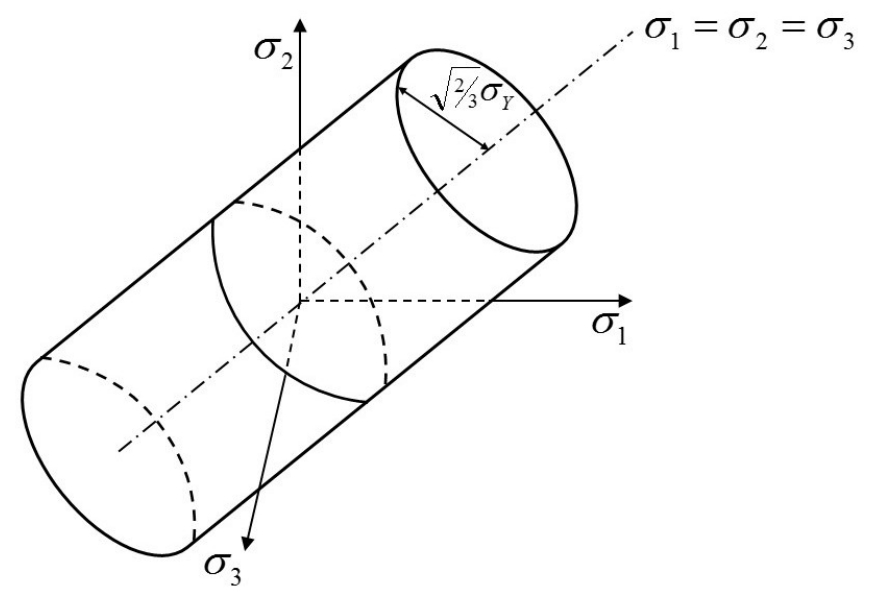

Figure 13: von Mises yield surface in 3-D stress space

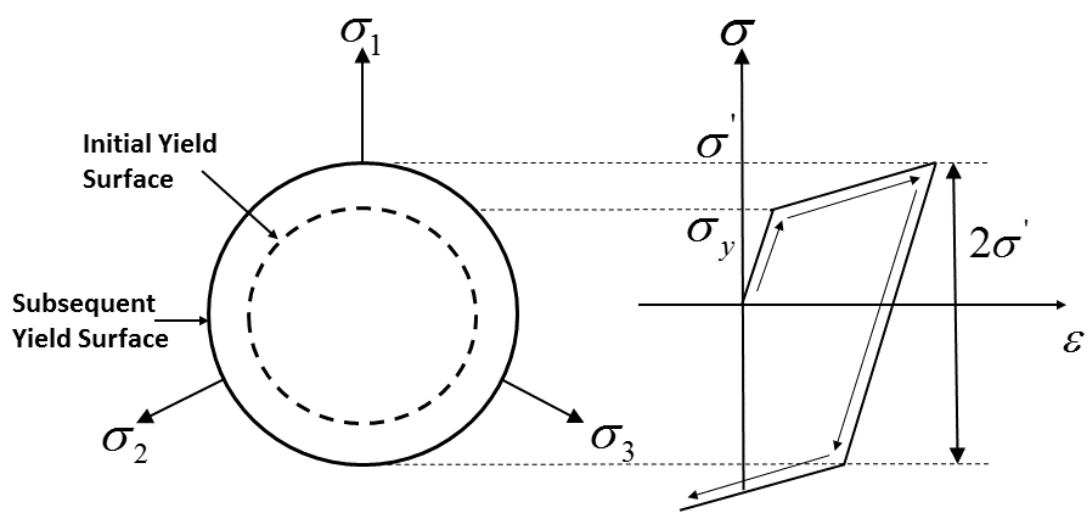

Figure 14: Isotropic hardening for von Mises yield surface with typical corresponding loading-unloading behavior

\section{Drucker Prager Criterion}

The most suitable yielding criterion for soils is the Mohr Coulomb model. In a 3-D stress space, it is represented by an irregular hexagonal pyramid, as shown in Fig. 15. 
The Drucker Prager model [144] is a smooth extension of the former, to transcend the numerical problems arising from the plastic flow at the corners of the hexagonal yielding surface. It is also more convenient for implementation in a computational framework. The yield criterion is given by [4, 130, 131, 145],

$$
f\left(I_{1}, J_{2}\right)=\sqrt{J_{2}}+\alpha I_{1}-k=0
$$

or

$$
f(\sigma)=\sigma_{e q}+\alpha \sigma_{k k}-\sigma_{y}
$$

The Drucker-Prager yielding surface is expressed in terms of the the second invariant of the deviatoric stress tensor $J_{2}$, the first invariant of the stress tensor $I_{1}$, and two material constants $\alpha$ (effective friction) and $k$ (effective cohesion) [146]. The presence of mean stress (through $I_{1}$ ) ensures the dependence of yield surface on hydrostatic pressure and hence implies volumetric dilation in addition to distortion. Therefore, it can also be considered as a modified version of the von Mises model. Isotropic

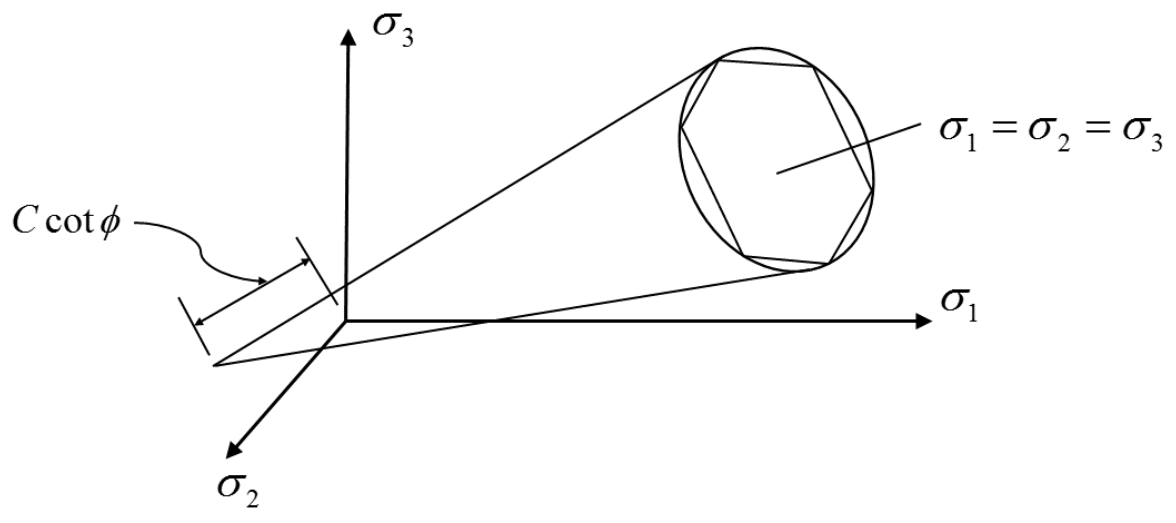

Figure 15: Drucker-Prager and Mohr-Coulomb Yield Surfaces

hardening can be incorporated in the model through the following equation $[4,130$, 131, 145]:

$$
f=\sqrt{J_{2}}+\alpha I_{1}-\sigma_{y}(\kappa)
$$




$$
\sigma_{y}(\kappa)=k+H \kappa
$$

The plastic flow potential $g$, for Drucker-Prager model is given as [145],

$$
g=\sqrt{J_{2}}+\beta I_{1}
$$

However, as discussed earlier, we will adopt an associative rule for our simulation,

i.e., $\beta=\alpha$, which implies that the angle of dialation $\psi$ is equal to the angle of internal friction $\phi$. The yield surface in Eq. 69 forms a smooth circular cone in a 3-D stress space, as shown in Fig. 15. The assumption that the Mohr-Coulomb yield surface is circumscribed by the Drucker-Prager yield surface, yields the expressions for $\alpha$ and $k$ as [16]:

$$
k=\frac{6 c \cos \phi}{\sqrt{3}(3 \pm \sin \phi)} ; \quad \alpha=\frac{2 \sin \phi}{\sqrt{3}(3 \pm \sin \phi)}
$$

where $c$ is the cohesion. The symbol \pm (in the denominator of the above equation) is positive or negative based on whether the Drucker Prager yield surface passes through the inner or outer apexes of the Mohr-Coulomb yield surface. We choose the -ve variation for our model. Chin and Mizuno [143], and Xu et al. [28] discuss the advantages and disadvantages of the Drucker-Prager model in detail.

\section{Discussion: Yield Surfaces and Plastic Flow Rules in Geomechanics}

In practice, the associated flow is a more popular and convenient approach than nonassociated flow, and has been used extensively for all types of materials including metals, soil, rock and concrete. For dynamic soil analysis too, it has been successfully implemented in many studies $[16,25,28,145,147]$. Despite the popularity of the associated flow rule, it is not always considered to be the best for soil models. This is 
because some elasto-plastic models like the associated von Mises, do not take 'volumetric dilation' of the material into consideration, and are therefore termed as volume preserving or isochoric. We know that many soils do exhibit volumetric straining, and thus an associated Drucker-Prager model can be used to include the effect of volumetric dilation. However, for granular soils, this change in volume may be overestimated by the associated Drucker-Prager model, while a non-associative law is capable of preserving the volume and limiting it to a realistic level - a behaviour more suited for granular materials $[148,149]$. Therefore, many studies employ non-associative models, especially for granular soils.

At the same time, the associated Drucker Prager model seems a reasonable approximation for clayey soils. As a first step, even the associated von Mises criterion can be implemented. In 2012, a USGS supported study conducted by the Quake Group at Carnegie Mellon University [133] successfully used the associated von Mises and Drucker-Prager model with isotropic hardening, for inelastic ground motion analysis.

Even the non-associative models are not free of criticism and have their own shortcomings. For example, in a non-associative model, the originally symmetric stiffness matrix may lose its symmetric nature due to the flow rule not being associated with the yield function. Non-symmetric solvers are required for computation of such problems [3]. Sandler and Pucik $[150,151]$ also questioned the mathematical validity of such type of flow potential function and showed that the non-associative models are not stable due to the "inadmissibly generating unbounded energy from quiescent states". A Sandia National Laboratory report released in 2004 by Fossum and Brannon [152] also highlighted the same concern. Therefore, even though the use of associative flow rules may appear convenient implementation-wise, there are much more profound mathematical reasons for its usage. It ensures well-posed boundary value problems, uniqueness of the solution and preserves the validity of the so called 
stability postulates [143].

With the existence of so many material models defined specifically for different types of applications, the skill lies in judging when is it appropriate to use them, and then deciding the values for parameters. Higher complexity of model does not necessarily imply improved accuracy since the simulation results depend critically on the quality of the physical data used. Infact, $\mathrm{Xu}$ [16] and $\mathrm{Xu}$ et al. [28] stated that for over-consolidated soils, the behaviour of the soils is mainly controlled by the part of the perfectly plastic yielding surface, and therefore no hardening was assumed in the study. Similar approach was adopted by Dupros et. al [25], and a perfectly plastic model was implemented to circumvent the difficulty posed by existence of too many parameters. At this point, we also recall that the intent of this study is to quantify the uncertainty in non-linear seismic wave propagation, and for an overly complex elasto-plastic model, a major obstacle will surface in form of the difficulty in model parameter identification and lack of knowledge of soil properties.

Concluding the discussion, we restrict our focus to the simulation of seismic wave propagation in an elasto-plastic soil model represented by rate-independent, associated von Mises and Drucker-Pracker plastic flow, with isotropic hardening.

\section{Elasto-plastic Tangent Moduli}

The derivation noted below closely follows the description provided in $[4,130]$. The consistent tangent modulus is derived from the continuum rate equations through the implementation of Kuhn Tucker consistency condition, that forces the stress point to stay on the yield surface after the plastic flow begins. The simulation begins with the application of a load increment that results in an increment in the displacement and the total strain. This stress increment is related to the strain increment through a constitutive relationship. Recalling the relationships mentioned earlier in section 
3.6.2,

$$
\begin{gathered}
d \sigma_{i j}=C_{i j k l} d \epsilon_{k l} \\
\Delta \boldsymbol{\sigma}=\boldsymbol{C}\left(\Delta \epsilon-\Delta \epsilon^{p}\right) \\
\Delta \epsilon^{p}=\Delta \Lambda \frac{\partial g}{\partial \boldsymbol{\sigma}}
\end{gathered}
$$

where $\partial g / \partial \boldsymbol{\sigma}$ is evaluated at the beginning of the increment. Using Eq. 77 and 76, we get:

$$
\Delta \boldsymbol{\sigma}=\boldsymbol{C} \Delta \epsilon-\Delta \Lambda \boldsymbol{C} \frac{\partial g}{\partial \boldsymbol{\sigma}}
$$

Using the Kuhn-Tucker consistency conditions with the above equation, we get the expression for increment $\Delta \Lambda$ :

$$
\Delta \Lambda=\frac{\left(\frac{\partial f}{\partial \boldsymbol{\sigma}}\right)^{T} \boldsymbol{C} \Delta \epsilon}{\left(\frac{\partial f}{\partial \boldsymbol{\sigma}}\right)^{T} \boldsymbol{C} \frac{\partial g}{\partial \boldsymbol{\sigma}}}
$$

Substituting Eq. 79 in 78,

$$
\Delta \boldsymbol{\sigma}=\boldsymbol{C}\left(\Delta \epsilon-\frac{\left(\frac{\partial f}{\partial \boldsymbol{\sigma}}\right)^{T} \boldsymbol{C} \Delta \epsilon \frac{\partial g}{\partial \boldsymbol{\sigma}}}{\left(\frac{\partial f}{\partial \boldsymbol{\sigma}}\right)^{T} \boldsymbol{C} \frac{\partial g}{\partial \boldsymbol{\sigma}}}\right)
$$

The incremental stress-strain relationship in plastic range is given as,

$$
\begin{aligned}
\Delta \boldsymbol{\sigma} & =\boldsymbol{C}_{e p} \Delta \epsilon \\
& =\left(\boldsymbol{C}+\boldsymbol{C}^{p}\right) \Delta \epsilon
\end{aligned}
$$

where $\boldsymbol{C}_{\boldsymbol{e} \boldsymbol{p}}$ is the tensor of the tangent elasto-plastic moduli and $\boldsymbol{C}^{\boldsymbol{p}}$ is the plastic stiffness matrix. By rearranging Eq. 80 and adding the linear hardening parameter 
$H$, we have:

$$
\Delta \boldsymbol{\sigma}=\left(\boldsymbol{C}-\frac{\boldsymbol{C} \frac{\partial g}{\partial \boldsymbol{\sigma}}\left(\frac{\partial f}{\partial \boldsymbol{\sigma}}\right)^{T} \boldsymbol{C}}{\left(\frac{\partial f}{\partial \boldsymbol{\sigma}}\right)^{T} \boldsymbol{C} \frac{\partial g}{\partial \boldsymbol{\sigma}}+H}\right) \Delta \epsilon
$$

Therefore, the consistent tangent can be computed by,

$$
\boldsymbol{C}_{\boldsymbol{e p}}=\left(\boldsymbol{C}-\frac{\boldsymbol{C} \frac{\partial g}{\partial \boldsymbol{\sigma}}\left(\frac{\partial f}{\partial \boldsymbol{\sigma}}\right)^{T} \boldsymbol{C}}{\left(\frac{\partial f}{\partial \boldsymbol{\sigma}}\right)^{T} \boldsymbol{C} \frac{\partial g}{\partial \boldsymbol{\sigma}}+H}\right)
$$

\subsubsection{Computational Plasticity}

The formulation and implementation of numerical algorithms to solve the global equilibrium equations coupled with local plasticity equations, form the computational framework for solving elasto-plastic problems. We shall use the finite element method to solve the weak form of the problem under consideration. At the constitutive level, the general algorithm for elasto-plastic analysis, which enables the computation of stress tensor and its linearisation, is strain driven and quite complicated. It can be inferred from Eq. 83, that the involved non-linearity requires an iterative scheme because, "the current state and an increment is known, but the effect of the increment depends on the state that the increment gives rise to" [4].

\section{Return Mapping Algorithm}

The return-mapping algorithm [130] maps the stress onto the yield surface, and is at the core of computational plasticity. Given the stress, the strain increment, and other internal parameters, a return mapping algorithm evaluates the final values of stress and the internal parameters at each quadrature point. As long as the strain remains within the elastic region, an elastic analysis can be performed to evaluate the final stress. However, when the strain increment causes a plastic response, the final stress (and internal parameters) can be determined using a return-mapping algorithm. This 
is accomplished by using a so-called trial stress which is actually impossible to obtain in a real physical situation. A Newton-Raphson method is then used to let this trial stress "flow" back to the admissible region, i.e., on the yield surface. The closest point projection method (popular return mapping approach for plasticity problems), described in [130] has been implemented in FEniCS Solid Mechanics Library [153].

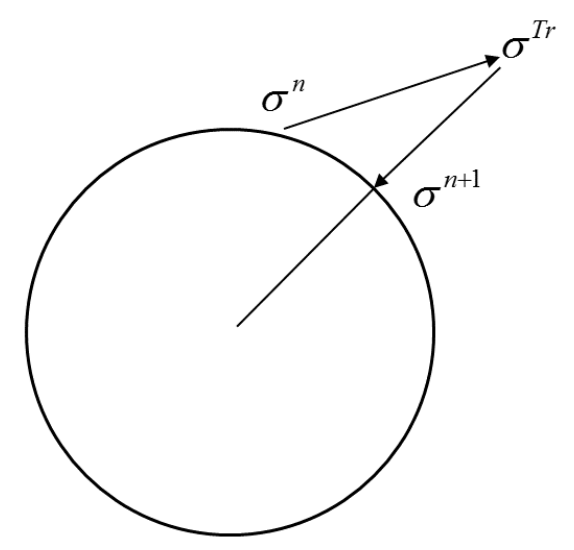

Figure 16: Schematic of return mapping

\subsubsection{Finite Element Method: Weak form}

For a plasticity problem, the linear and bilinear form of the variational equation are obtained through the linearisation of the balance of momentum equation. Before we move on to dynamic plasticity, let us revisit the weak form for dynamic linear elastic case. The standard balance of linear momentum equation for the body $\Omega$ reads:

$$
\rho \ddot{u}-\nabla \cdot \sigma=f
$$

where $f$ is the body force, $\rho$ is the material density and $\ddot{u}$ is the acceleration. The previously derived weak form given by Eq. 41 is

$$
\int_{\Omega} \rho \ddot{u} \cdot v d x+\int_{\Omega} \sigma: \nabla v d x-\int_{\partial \Omega} h \cdot v d s-\int_{\Omega} f \cdot v d x=0
$$


The above noted variational form was implemented earlier for linear elastic wave propagation with Newmark Beta time discretization using FEniCS.

For a quasi-static elasto-plastic problem, we rewrite our basic PDE, i.e., Eq. 84 without the inertia term [154]:

$$
-\nabla \cdot \sigma=f
$$

This can be alternatively written as

$$
-\nabla \cdot \sigma^{n+1}=f^{n+1}
$$

It is also known that

$$
\sigma^{n+1}=d \sigma+\sigma^{n}
$$

Using the above two relations, we get

$$
\begin{gathered}
-\nabla \cdot\left(d \sigma+\sigma^{n}\right)=f^{n+1} \\
-\nabla \cdot d \sigma=f^{n+1}+\nabla \cdot \sigma^{n}
\end{gathered}
$$

Now we multiply Eq. $89 \mathrm{~b}$ with a test function $v$, and because $\sigma^{n}$ is known, we move it to the right hand side. Using Eq. 81, and replicating the steps in derivation of Eq. 85, we attain the following non-linear form and Jacobian for a quasi-static elasto-plastic problem [155]:

$$
\begin{aligned}
a(v, u) & =\int_{\Omega} \nabla v: C_{e p}: \nabla^{s} u d x \\
L(v) & =\int_{\Omega} \sigma: \nabla v d x-\int_{\Omega} f \cdot v d x-\int_{\Gamma} h \cdot v d s
\end{aligned}
$$


where $u$ is the displacement field increment, $C_{e p}$ is the consistent tangent operator, and $\sigma$ is the stress from the previous iteration. The dynamic weak form for elastoplasticity shall also include the inertia term (as in Eq. 85) and its linearisation, both of which were not a part of the quasi-static weak form derived above. To implement the dynamic plasticity problem, we use a staggered time stepping scheme [156] based on the implicit Newmark Beta method introduced earlier. While the basics remain the same, this scheme differs from the standard Newmark Beta algorithm used earlier for simulating linear elastic wave propagation. The procedure assumed by Newmark Beta temporal discretization gives the velocity and displacement as:

$$
\begin{aligned}
& \dot{u}^{n+1}=\dot{u}^{n}+\frac{\Delta t}{2}\left(\ddot{u}^{n}+\ddot{u}^{n+1}\right) \\
& u^{n+1}=u^{n}+\Delta t \dot{u}^{n}+\frac{1-2 \beta}{2} \Delta t^{2} \ddot{u}^{n}+\beta \Delta t^{2} \ddot{u}^{n+1}
\end{aligned}
$$

It can be noted that the determination of the new acceleration $\ddot{u}^{n+1}$ requires the knowledge of the displacement $u^{n+1}$. We now introduce the displacement prediction at time $t=t^{n+1}$ as:

$$
\hat{u}^{n+1}=u^{n}+\Delta t \dot{u}^{n}+\frac{1-2 \beta}{2} \Delta t^{2} \ddot{u}^{n}
$$

Using the above expression along with Eq. 91b, we obtain the equation for acceleration $\ddot{u}^{n+1}$ and substituting this expression in Eq. 85, we get the non-linear form of dynamic elasto-plastic problem:

$$
L(v)=\int_{\Omega} \rho \frac{u^{n+1}-\hat{u}^{n+1}}{\beta \Delta t^{2}} \cdot v d x+\int_{\Omega} \sigma: \nabla v d x-\int_{\Gamma} h \cdot v d s-\int_{\Omega} f \cdot v d x=0
$$

Now taking the Jacobian of Eq. 93, with trial function $d u$, we get the complete weak form:

$$
a(v, d u)=\int_{\Omega} \frac{\rho}{\beta \Delta t^{2}} d u \cdot v d x+\int_{\Omega} \nabla v: C_{e p}: \nabla^{s} d u d x
$$


The algorithm for implementation of the staggered time stepping scheme is shown as Algorithm 1 along with the UFL (Unified form language) script for a 3-D dynamic elasto-plastic problem.

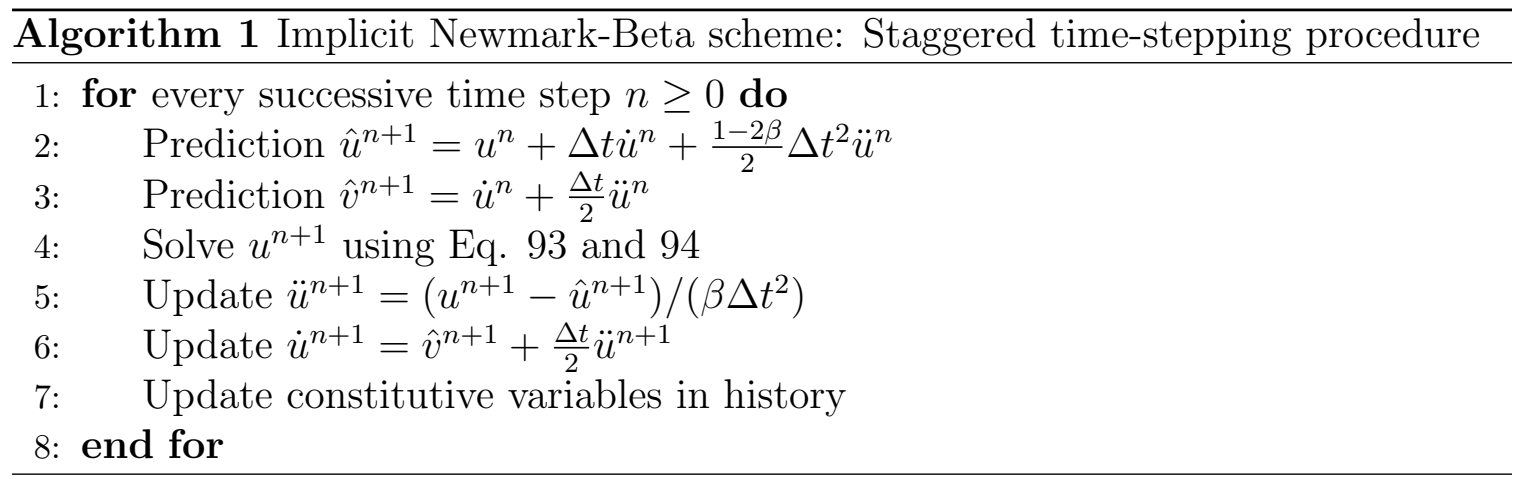

Now, we attempt to add a simple damping term similar to Eq. 43, to the derived weak form. A damping constant $\eta$ is multiplied to the rate of strain, and the test function to create damping in the model.

$L(v)=\int_{\Omega} \rho \frac{u^{n+1}-\hat{u}^{n+1}}{\beta \Delta t^{2}} \cdot v d x+\int_{\Omega} \eta \operatorname{tr}(\dot{\epsilon}) \cdot \operatorname{tr}(\nabla v) d x+\int_{\Omega} \sigma: \nabla v d x-\int_{\Gamma} h \cdot v d s-\int_{\Omega} b \cdot v d x$

We know that $\dot{\epsilon}$ can be written as $\nabla \dot{u}^{n+1}$, i.e., gradient of the unknown velocity. This unknown velocity should be expressed in terms of unknown displacement and therefore, by substituting step 5 into step 6 of Algorithm 1, we obtain the following non-linear form:

$$
\begin{aligned}
L(v)=\int_{\Omega} \rho \frac{u^{n+1}-\hat{u}^{n+1}}{\beta \Delta t^{2}} \cdot v d x+\int_{\Omega} \eta \operatorname{tr} & \left(\frac{\nabla u^{n+1}}{2 \beta \Delta t}-\frac{\nabla \hat{u}^{n+1}}{2 \beta \Delta t}+\nabla \hat{v}^{n+1}\right) \cdot \operatorname{tr}(\nabla v) d x \\
& +\int_{\Omega} \sigma: \nabla v d x-\int_{\Gamma} h \cdot v d s-\int_{\Omega} b \cdot v d x
\end{aligned}
$$


Deriving the Jacobian of Eq. 96 with trial function $d u$, we can get the bilinear part of the weak form:

$a(v, d u)=\int_{\Omega} \frac{\rho}{\beta \Delta t^{2}} d u \cdot v d x+\int_{\Omega} \eta \operatorname{tr}(\nabla v) \cdot \operatorname{tr}\left(\frac{1}{2 \beta \Delta t} \nabla d u\right) d x+\int_{\Omega} \nabla v: C_{e p}: \nabla^{s} d u d x$

1$$
2
$$

5

6

7

8

11

16

18

19

20

\# UFL (Unified form language) script for a 3-D dynamic elasto-plastic problem 


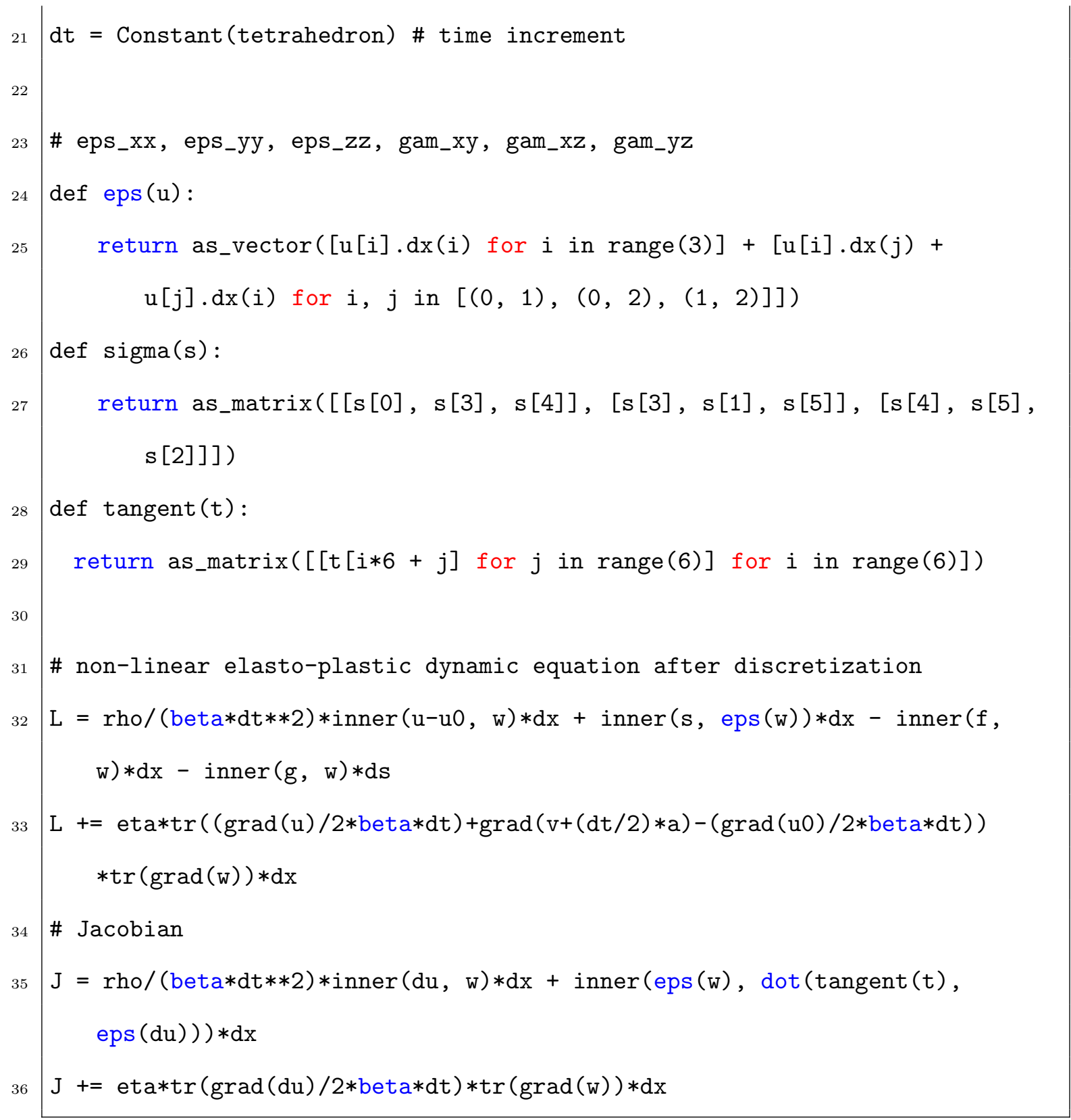

For a weak, medium and strong Gaussian pulse source, i.e., increasing values of $\beta$ in Eq. 44, a comparison has been shown in Fig. 17, 18, and 19 between 2-D wave propagation in a linear elastic and an elasto-plastic Drucker-Prager, isotropic homogeneous subsurface material. The values chosen for physical parameters are:

$$
G=25 M P a, \quad \nu=0.3, \quad \rho=1700 \mathrm{~kg} / \mathrm{m}^{3}
$$




$$
c=6 k P a, \quad \phi=23.5^{\circ}, \quad \eta=5 \%, \quad H=0.1 E
$$

where $G$ represents the shear modulus, $c$ is the cohesion, $\phi$ is the angle of friction and $H$ is the linear hardening parameter. These values are representative of the slightly over consolidated soil type found in Ottawa valley. The simulations have been performed on a 2 -D square mesh of size $[1,1] \mathrm{km}^{2}$ discretised into triangular elements of second order with six nodes per element, and the magnitude has been calculated as the square root of the sum of the squares of the $x$ and $y$ component of particle displacement. A free boundary has been assumed for the ground surface and a reflective condition has been implemented on rest of the boundary for the 2-D domain. Similar boundary conditions have been used in this study for all the 2-D analysis conducted hereafter. A mesh convergence study was conducted to find the optimum mesh resolution that provides a balance between the numerical accuracy and the required computational effort. A mesh resolution of $\Delta h=3$ for all square meshes of length $1 \mathrm{~km}$ has been used in the linear elastic and inelastic wave propagation simulations shown throughout this study. It was observed that for a lower mesh resolution, the solution becomes pixelated and coarse while the wave profile and magnitude remain nearly identical. On the other hand, refining the mesh further doesn't cause any noticeable change or smoothness in the solution. The $c g$ (conjugate gradient) solver has been used to solve the linear elastic problem while the gmres (generalised minimal residual) solver along with a hypre amg (algebraic multi-grid) pre-conditioner has been used for the elasto-plastic model.

There are noticeable differences in the linear and the non-linear simulations even though a similar pattern is evident. In Fig. 17, it can be observed that the linear and non-linear simulations exhibit almost similar wave propagation patterns 


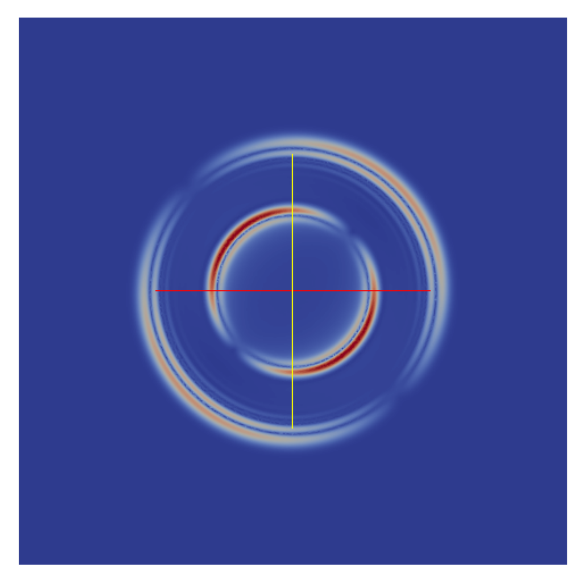

(a) $t=1.2 \mathrm{~s}$

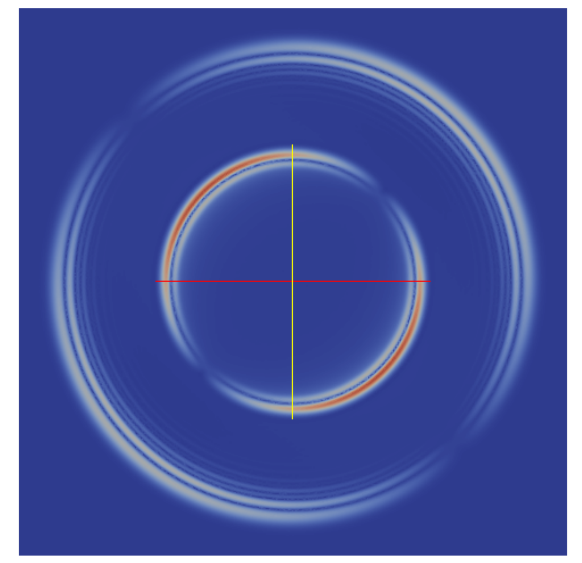

(c) $t=1.9 \mathrm{~s}$

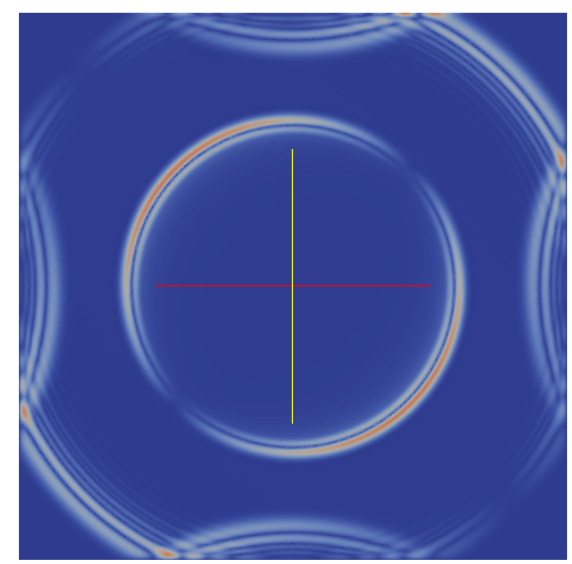

(e) $t=2.5 \mathrm{~s}$

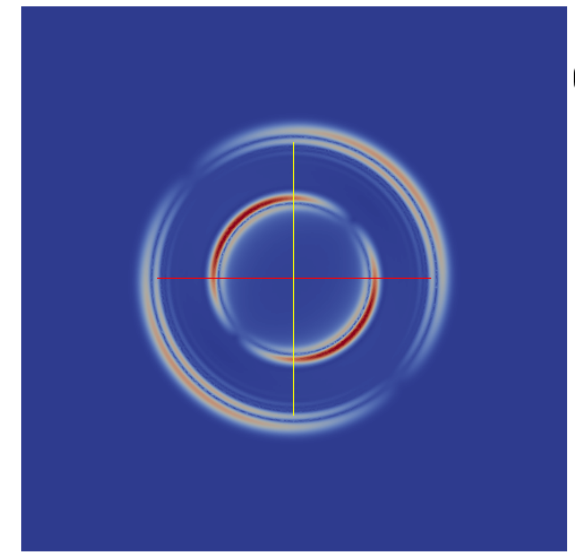

(b) $t=1.2 \mathrm{~s}$

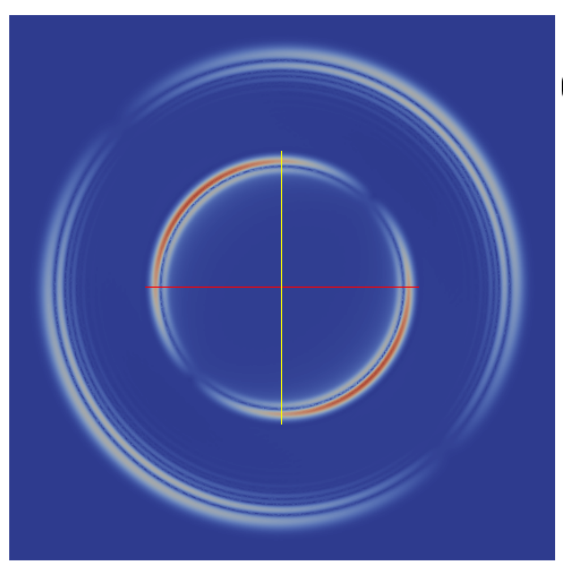

(d) $t=1.9 \mathrm{~s}$

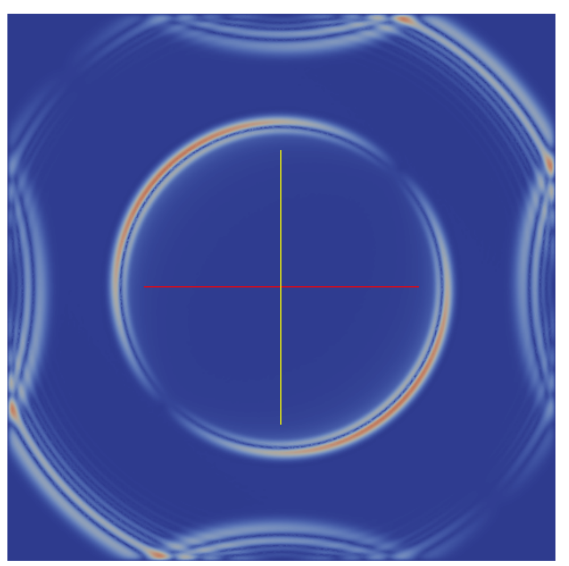

(f) $t=2.5 \mathrm{~s}$

\section{$\mathrm{km}$}

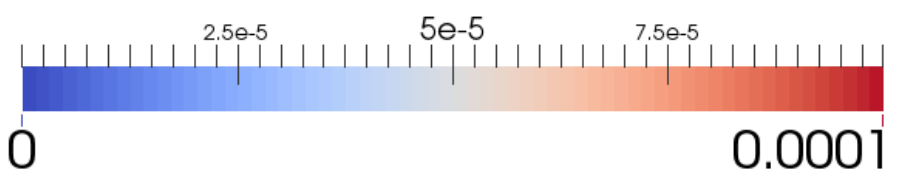

Figure 17: Magnitude of waves propagating in a homogeneous isotropic elastic (left) and elasto-plastic Drucker Prager (right) medium for a weak source 


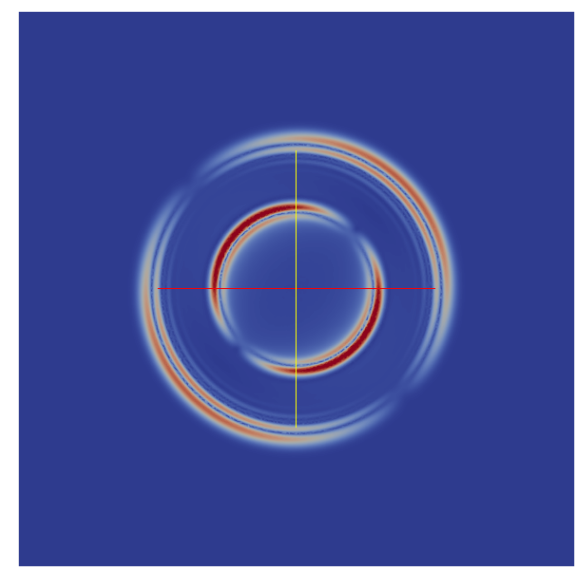

(a) $t=1.2 \mathrm{~s}$

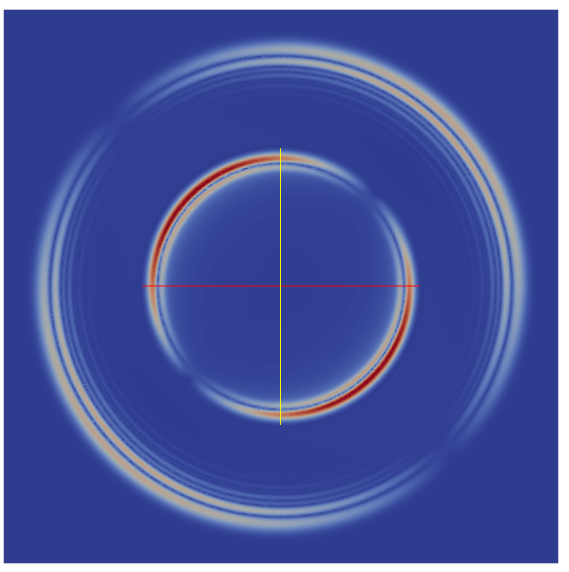

(c) $t=1.9 \mathrm{~s}$

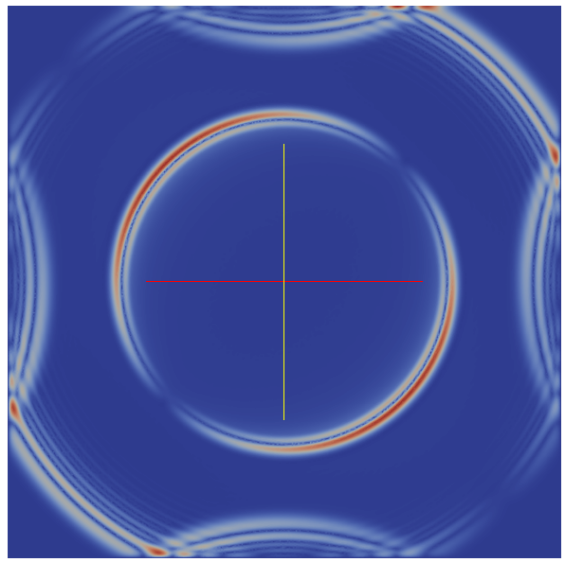

(e) $t=2.5 s$

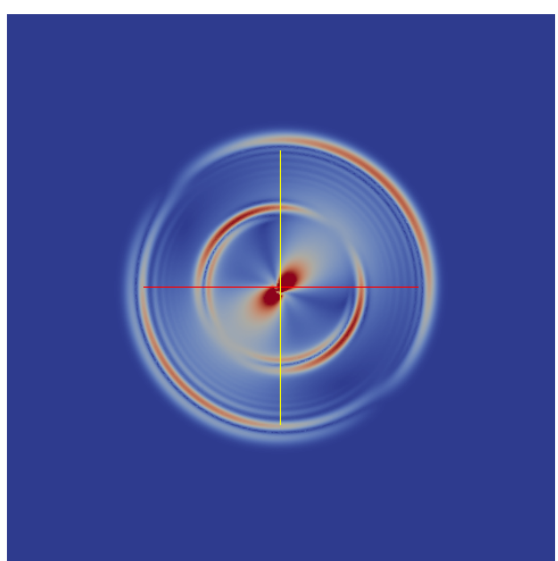

(b) $t=1.2 \mathrm{~s}$

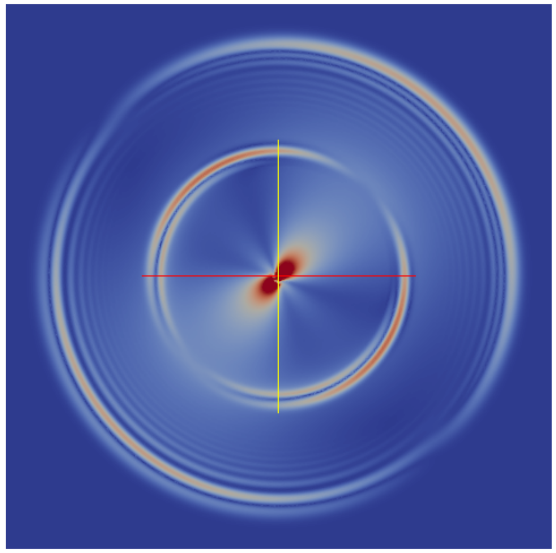

(d) $t=1.9 \mathrm{~s}$

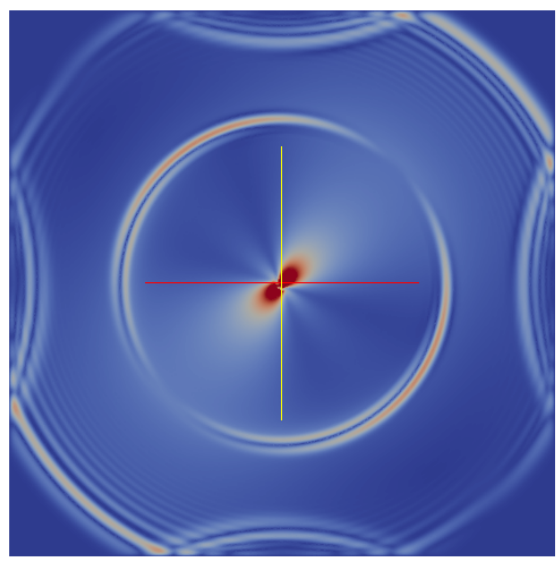

(f) $t=2.5 \mathrm{~s}$

\section{$\mathrm{km}$}

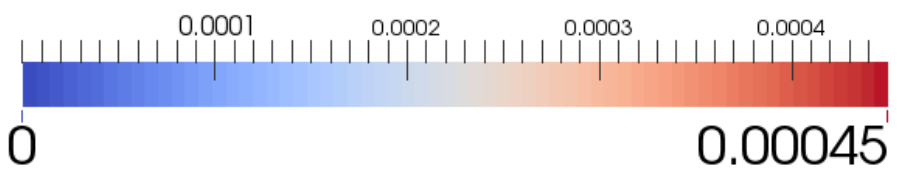

Figure 18: Magnitude of waves propagating in a homogeneous isotropic elastic (left) and elasto-plastic Drucker Prager (right) medium for a source of medium strength 


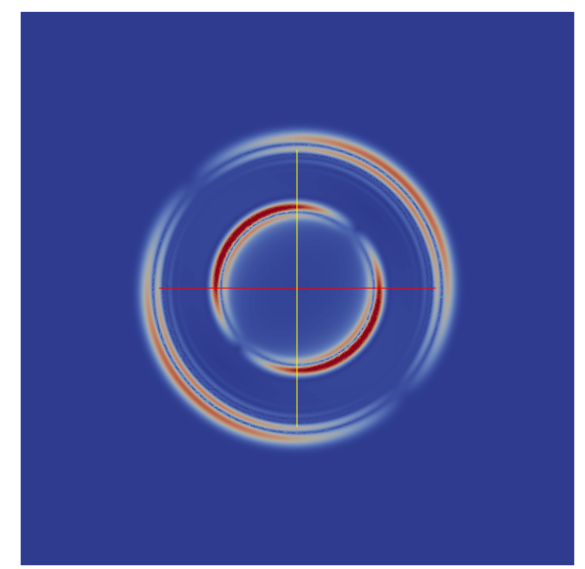

(a) $t=1.2 \mathrm{~s}$

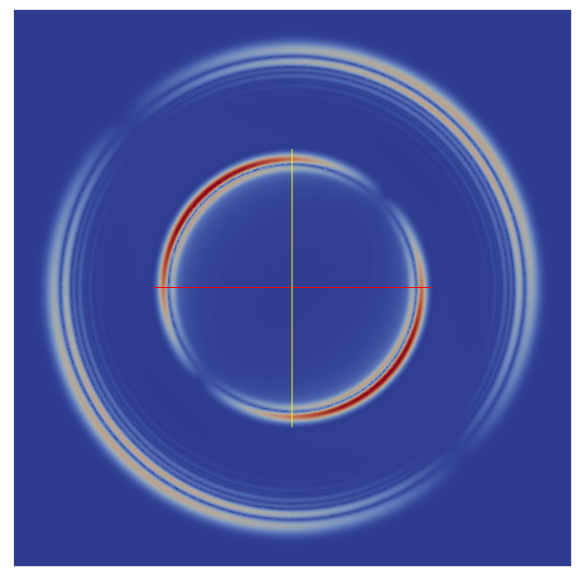

(c) $t=1.9 \mathrm{~s}$

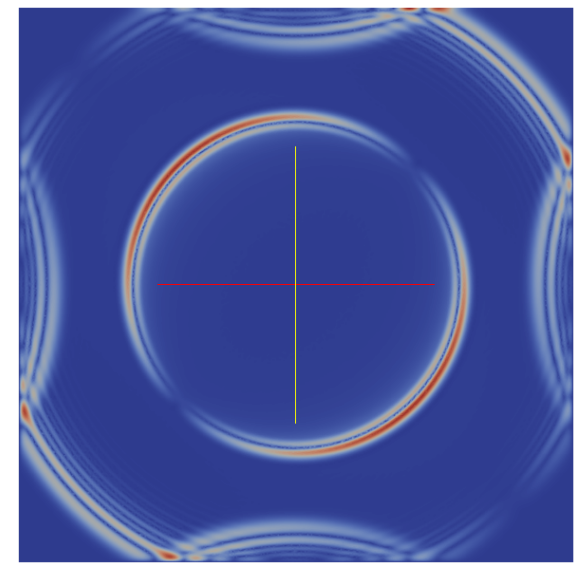

(e) $t=2.5 \mathrm{~s}$

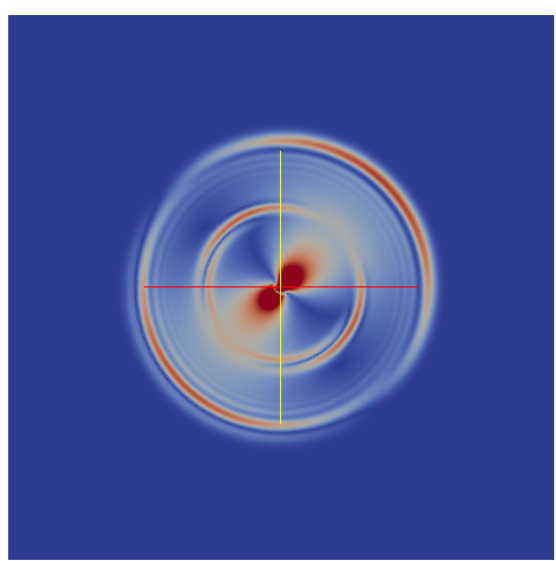

(b) $t=1.2 \mathrm{~s}$

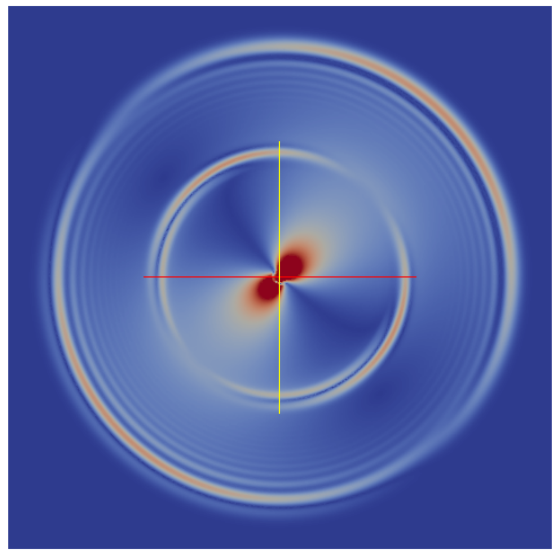

(d) $t=1.9 \mathrm{~s}$

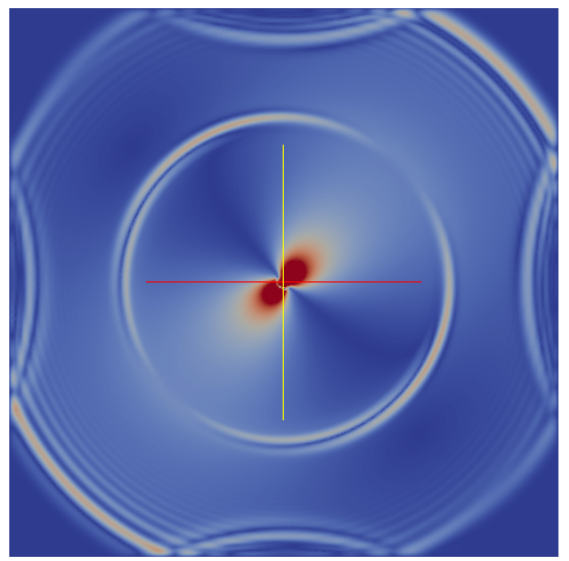

(f) $t=2.5 \mathrm{~s}$

$\mathrm{km}$

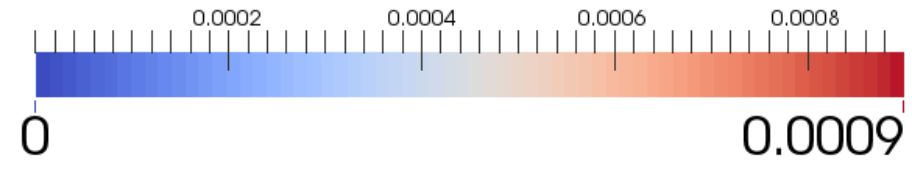

Figure 19: Magnitude of waves propagating in a homogeneous isotropic elastic (left) and elasto-plastic Drucker Prager (right) medium for a strong source 
and even the magnitude values appear to be very close. This corroborates the fact that for a weak loading, the contribution of non-linear effects will be minimal and therefore, almost similar results will be observed for both the cases. However, in Fig.18 and 19, linear results show higher magnitude in distinct wave profiles while non-linear simulations show permanent displacement, which is particularly high near the centre of the mesh that is also the location of major plastic deformation, given that the pulse originated there. This is in agreement with the common findings in the literature $[16,145]$ since permanent plastic deformation takes place in elasto-plastic media.

\section{Validation of the computational model}

The computational model for dynamic elasto-plasticity has been validated by comparing the response for a small-scale test problem with that from a published open source plasticity code presented in [132] (refer Page 73). The published code is based on a backward Euler scheme for a rate-independent associated von Mises model with kinematic hardening (VM-KH), while we implement a Drucker Prager model with isotropic hardening (DP-IH) based on the Newmark Beta scheme.

Through this validation, we also demonstrate the capability of our model to solve 3-D problems. To validate our model, we model the same problem with similar values for physical parameters, as considered in [132]. We consider a uniaxial tensile testing problem where a quadratic beam is under a displacement controlled mechanical loading. Left end of the beam is clamped while cyclic loading is set on the right end in the form of a time dependent Dirichlet BC. The beam has been discretised into 3-D tetrahedral elements of first order with four nodes per element. The following values 


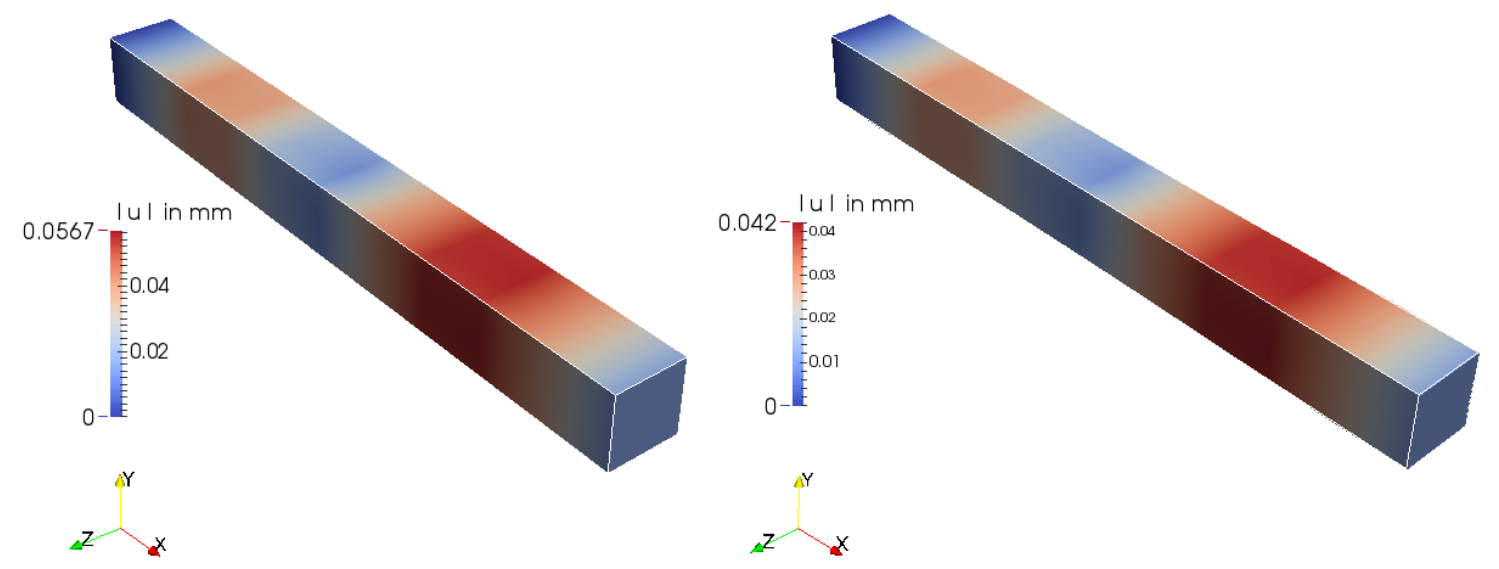

Figure 20: Comparison of Deformation: 1) Response of VM-KH model from referred code (left); 2) Response of developed DP-IH model (right)

were chosen for various physical parameters:

$$
E=200000 M P a, \quad \nu=0.3, \quad \rho=8.3 * 10^{-9} t / \mathrm{mm}^{3}, \quad \sigma_{y}=300 M P a, \quad H=0.1 E
$$

where $\sigma_{y}$ is the yield stress and $H$ is the hardening parameter. A time step of $0.05 s$ was assumed for a 3-D mesh with dimensions $[100,10,10] \mathrm{mm}^{3}$. Resulting simulations have been compared in Fig. 20, where the magnitude of displacement $u$ (in $\mathrm{mm}$ ) has been shown and it can be observed that for a simple uniaxial problem, response from both the simulations is quite similar. Results from the von Mises model exhibit slightly larger displacement values because of the larger plastic strain needed to accommodate the low yield levels assumed [145]. Some discrepancy in output is also expected due to the different hardening models and time stepping schemes implemented in the models in addition to the fundamental difference in the yield criteria. 


\subsubsection{Large-scale simulation using high performance comput- ing}

A finite element system of non-linear equations (noted in the previous section) comprising of more than 100 million dof was solved to demonstrate the large-scale parallel processing capability of the developed computational model. Initially developed on a desktop computer for a small scale problem, the code was later scaled and deployed on the Guillimin supercomputer (at McGill University) with 1040 parallel processes distributed over 65 nodes with $92 \mathrm{~GB}$ of memory per node. A square mesh of size $[1,1] \mathrm{km}^{2}$ was used to perform wave propagation simulation for the Drucker Prager

plasticity model. A Gaussian pulse in space $f=\left(f_{x}, f_{y}\right)$ given by Eq. 44, was applied as a source at the centre of the mesh. The values chosen for physical parameters are:

$$
\begin{aligned}
& G=38.5 M P a, \quad \nu=0.3, \quad \rho=1700 \mathrm{~kg} / \mathrm{m}^{3} \\
& c=6 \mathrm{kPa}, \quad \phi=23.5^{\circ}, \quad \eta=5 \%, \quad H=0.1 E
\end{aligned}
$$

A typical output obtained from the large-scale simulation performed on Guillimin supercomputer has been shown in Fig. 21. 


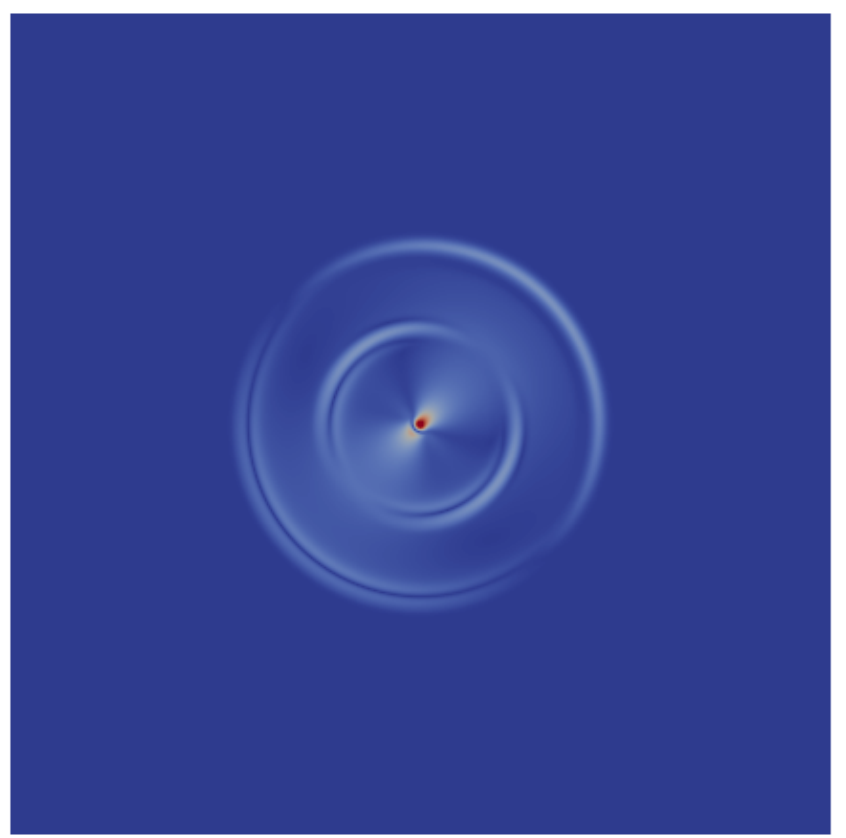

$\mathrm{km}$

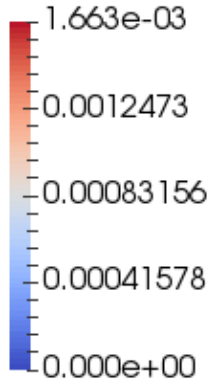

Figure 21: Magnitude of displacement shown for a large-scale simulation result (for a Drucker-Prager plasticity model) 


\section{Chapter 4}

\section{Uncertainty Quantification}

Uncertainty quantification primarily aims at defining the statistical characteristics of the uncertain input parameters and then propagating them through the computational model to characterize the random output. Since we implement the sampling based non-intrusive polynomial chaos expansion (PCE) for quantifying uncertainty in our model, a brief explanation has been provided in this chapter to familiarise the reader with the concept of sampling and $\mathrm{PCE}$.

\subsection{Monte Carlo Method}

Proposed in 1949 by Metropolis and Ulam [157], a popular approach followed to simulate stochastic models is the Monte Carlo simulation (MCS). Introduced as a means for modelling physical processes, solving combinatorial problems, and evaluating integrals, the concept was to feed a finite number of randomly generated values of the input (based on its statistical distribution) into the mechanistic models. With sufficiently large number of input values, the resulting output should have good statistical estimates (e.g. first and second order moments, i.e., mean and variance). The MCS technique uses the law of large numbers to provide accuracy, i.e, the difference 
between the expected and true response converges to zero for increasing number of realizations (samples).

However, it is not possible for the computer to create a genuine string of random numbers. Therefore, some programming environments like python and MATLAB [158] have in-built functions that can generate sequences of pseudo-random numbers.

To increase the accuracy of the estimate, unlimited number of new pseudo-random samples can be added to the existing ones. This is a major advantage of MC method. The convergence rate of MC simulation estimates is of the order $O\left(n^{-1 / 2}\right)$, where $n$ is the number of input samples used. Even though this is considered low, it can be observed that the convergence is not dependent on dimensionality of the random variables. This can prove to be very useful in cases involving multiple independent random variables.

Despite the robustness and simplicity of the Monte Carlo technique, its application for complex multi-dimensional models may sometimes be too computationally demanding and hence impractical in certain cases.

\subsubsection{Application}

If $X$ is a random variable of interest represented as a function of another random variable $\xi$, then $\xi$ can also be referred to as the germ. The number of MC simulations that should be run for a given model can't be clearly defined. Pseudo-random sampling of the germ $\xi$ can be done in MATLAB using functions like rand() (standard uniform distribution on the open interval $[0,1]$ ) and randn() (standard normal distribution with mean 0 and variance 1$)$.

Example. The following MATLAB code simulates the output of a function $Y$ which is a polynomial transform of the random variable $X$ having a Gaussian distribution 
with an expected value of 10 and standard deviation of 2 :

$$
\begin{aligned}
& Y=a X^{2} \quad \text { where } \quad X \sim N(10,4) \\
& X=\mu+\sigma \xi \quad \text { where } \quad \xi \sim N(0,1)
\end{aligned}
$$

We can analytically obtain the pdf of $Y$. The pdf estimated using kernel density estimate from MCS is plotted along with the analytically obtained pdf in Fig. 22. In this case, we had to use 10,000 samples to get a consistent estimate with reduced sampling error.

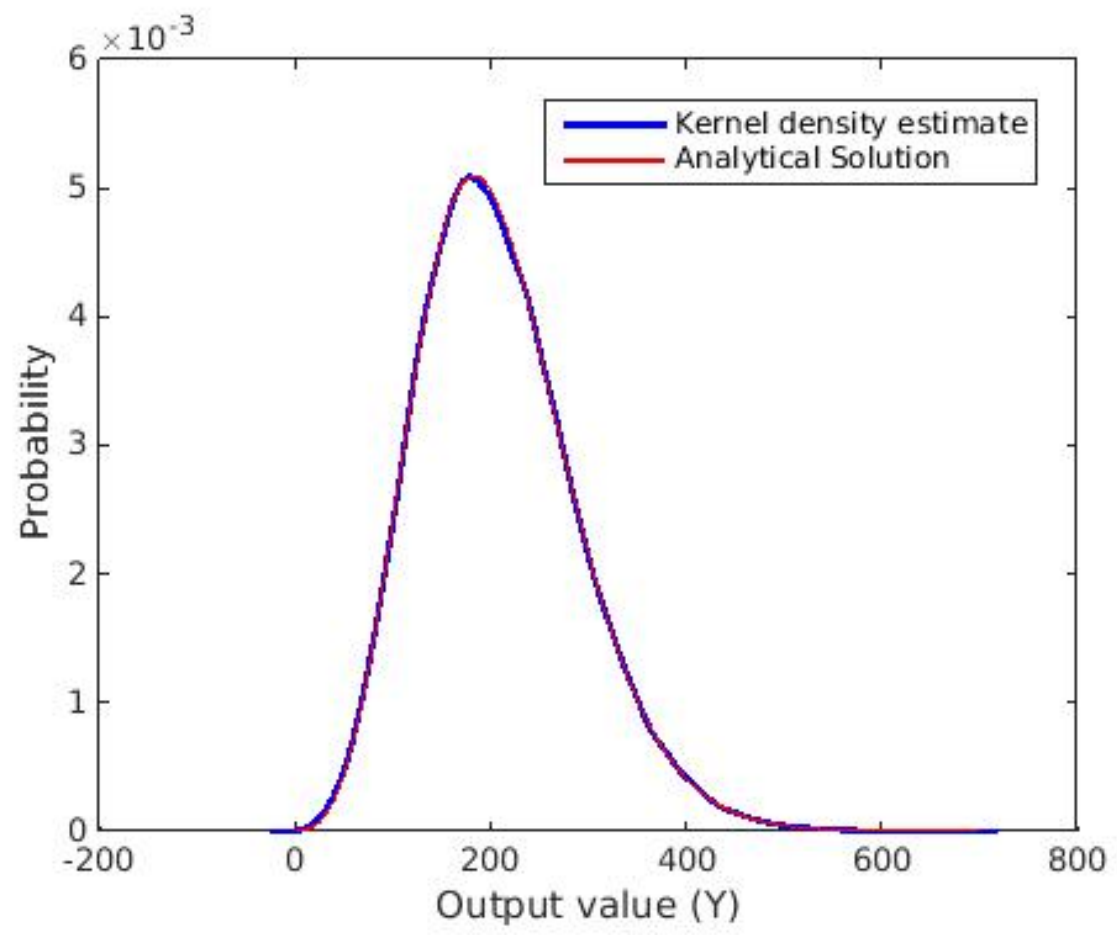

Figure 22: MC simulation vs. analytical solution for Eq. 98

\footnotetext{
$1 \quad \mathrm{a}=2$;

$2 \mathrm{mu}=10 ; \quad \%$ Mean

3 sigma $=2 ; \%$ Std. Deviation

$4 \mathrm{n}=10000 ; \%$ No. of Samples
} 


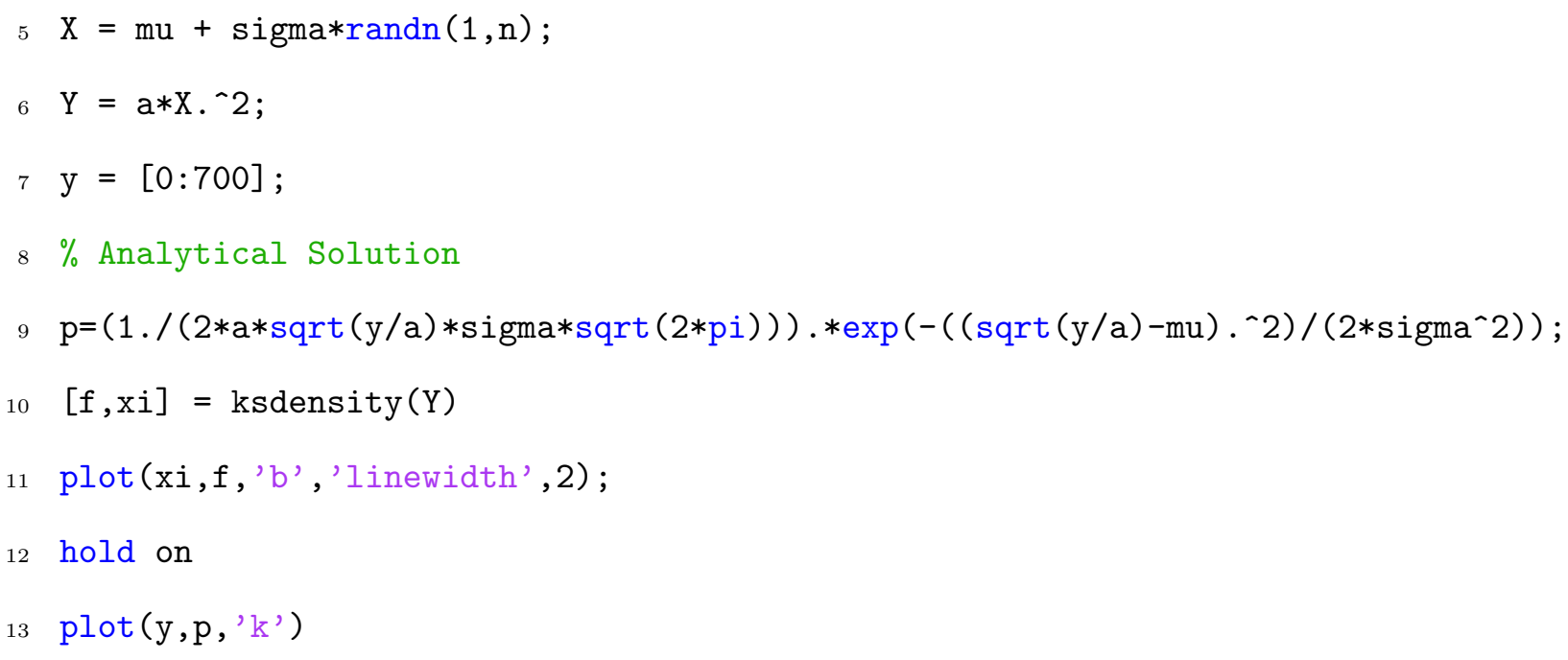

\subsection{Polynomial Chaos Expansion}

As noted in the previous section, the convergence of MCS is slow. This limitation can be overcome by using spectral methods, which are based on reconstructing the functional dependence of the solution on the random variables. Unlike $\mathrm{MC}$ technique, these methods are computationally less demanding and do not allow the "local nature of the input realizations to penalize the problem of determining the global variability of the solution" [6].

Wiener was the first to introduce polynomial chaos expansion (PCE) based on Hermite polynomials [159] for Gaussian random variables. Thereafter, Martin and Cameron [160] depicted that PCE was capable of approximating any random process with finite second-order moments. In concept, PCE represents a random variable as a function of another random variable with known distribution, and further represents that function as a polynomial expansion.

$$
Y=f(\xi)
$$


The method is used to expand the function $f$ in Eq. 99 as a polynomial series using a specific polynomial basis. The basis is a set of orthogonal polynomials which depends on the distribution of the random variable $\xi$.

\subsubsection{Orthogonal Polynomials}

Mathematically, orthogonality implies perpendicularity, i.e., if the dot product of two vectors is zero, they are orthogonal to each other. It is understood that the set of vectors $x, y, z$ form the basis for three dimensional space because they are orthogonal to each other.

$$
x . y=0 ; \quad x . z=0 ; \quad y . z=0,
$$

By definition, the functions, $f_{i}(x)$ and $f_{j}(x)$, are orthogonal on interval $[a, b]$ if their inner product is zero, i,.e,

$$
\int_{b}^{a} f_{i}(x) f_{j}(x) d x=0
$$

They are orthonormal if

$$
\int_{b}^{a} f_{i}(x) f_{j}(x) d x=\delta_{i j}
$$

where $\delta_{i j}$ is the Kronecker delta, i.e.,

$$
\begin{array}{rl}
\delta_{i j}=1 & i=j \\
& =0 \quad i \neq j
\end{array}
$$


Similarly, if $P_{n}(x)$ is a polynomial of degree $n$, then the polynomial set $\left[P_{n}(x), n \in N\right]$ is called orthogonal if it satisfies the following condition:

$$
\int_{b}^{a} P_{n}(x) P_{m}(x) W(x) d x=u_{n} \delta_{n m}, \quad n, m \in N
$$

where $W(x)$ is a specified weight function, $[a, b]$ is the interval of $P_{n}$ and $u_{n}$ are non zero constants. The polynomials are orthonormal if $u_{n}=1$. The inner product for two polynomial functions $f_{1}(x)$ and $f_{2}(x)$ can be defined by using the weight function $W(x)$ as

$$
\left\langle f_{1}, f_{2}\right\rangle_{W}=\int_{b}^{a} f_{1}(x) f_{2}(x) W(x) d x
$$

Also, if $W(x)$ is the weight function of a set of orthogonal polynomials $\left[P_{n}(x)\right]$ and it is also equal to the $p d f$ of a random variable $X$, then

$$
\left\langle P_{n}(X), P_{m}(X)\right\rangle_{W}=u_{n} \delta_{n m} \quad n, m \in N
$$

Such properties of the weight function apply to certain classes of orthogonal polynomials and random variables and are very critical for stochastic Galerkin schemes. The family of orthogonal polynomials used as basis function to span the $n$-dimensional stochastic space when the input uncertainty is Gaussian are known as Hermite Polynomials. To obtain the best statistical convergence, Legendre and Laguerre polynomials should be used as basis functions for uniform and exponential input distributions respectively. The classification used for mapping the distribution of the random variables to the optimal orthogonal polynomial is called the Askey scheme [161] and is shown in Table 1. 


\begin{tabular}{|l|l|l|l|}
\hline Distribution of $\xi$ & $\boldsymbol{P} \boldsymbol{D F}$ of $\xi$ & Univariate PC basis $\phi$ & Support $\Omega$ \\
\hline Gaussian & $\frac{1}{\sqrt{2 \pi}} \exp \frac{-\xi^{2}}{2}$ & Hermite Polynomials & $(-\infty,+\infty)$ \\
\hline Uniform & $\frac{1}{2}$ & Legendre Polynomials & {$[-1,1]$} \\
\hline Beta & $\frac{\xi^{\alpha}{ }^{1}(1-\xi)^{\beta}}{B(\alpha, \beta)}, \quad \alpha, \beta>0$ & Jacobi Polynomials & {$[-1,1]$} \\
\hline Gamma & $\frac{\xi^{\gamma} e^{1} \xi}{\Gamma(\gamma))}, \quad \gamma>0$ & Laguerre Polynomials & {$[0, \infty)$} \\
\hline
\end{tabular}

Table 1: Relationship between the type of Wiener-Askey polynomial [1] and the pdf of uncertain inputs

\subsubsection{Galerkin projection and Probability spaces}

The approximate solution of a stochastic PDE lies in a subspace, determined by an orthogonal basis. Then this approximate solution being sought can be obtained by minimizing the error between itself and the exact solution using an orthogonal projection onto this subspace. This is because the error is orthogonal to the chosen subspace. Therefore, curtailing the problem to a finite-dimensional subspace permits the computation of the solution as a finite linear combination of the basis functions in the subspace. This method is known as Galerkin projection. Similar technique is followed in PCE by using probability spaces. Such problems involving stochastic finite elements were first solved by Spanos and Ghanem [57, 162].

A probability space is a three-tuple measure space (with total measure one) which models the randomness of a system. It has three components $(\Omega, F, P)$, where $\Omega$ is called the sample space representing all possible outcomes, $F$ is the event space containing all possible events and a subset of $\Omega$, and $P: F \rightarrow \mathbb{R}$ is a function that assigns probabilities to the events in $F$. A random variable $X$ is therefore a function $X:(\Omega, F, P) \rightarrow \mathbb{R}$. 


\subsubsection{One Dimensional Polynomial Chaos Expansion}

Let us assume that a random variable $Y$ is the function of a $\mathrm{germ} \xi$ which is a standard normal random variable. As per Table 1, this implies that Hermite Polynomials should be used for PCE. Hermite polynomials are orthogonal with respect to the Gaussian measure and can be defined in a recursive way as follows:

$$
\psi_{n+1}(\xi)=\xi \psi_{n}(\xi)-n \psi_{n-1}(\xi)
$$

where,

$$
\begin{aligned}
& \psi_{0}(\xi)=1 \\
& \psi_{n}(\xi)=(-1)^{n} e^{\xi^{2} / 2} \frac{d^{n}}{d \xi^{n}} e^{-\xi^{2} / 2}, \quad n=1,2, \ldots \\
& \psi_{1}(\xi)=\xi \\
& \psi_{2}(\xi)=\xi^{2}-1 \\
& \psi_{3}(\xi)=\xi^{3}-3 \xi, \ldots
\end{aligned}
$$

The above set of polynomials form an orthogonal basis over $[-\infty, \infty]$ for weight function $w(\xi)=e^{-x^{2} / 2}$, with respect to the inner product

$$
\left\langle\psi_{i} \psi_{j}\right\rangle=\frac{1}{\sqrt{2 \pi}} \int_{-\infty}^{\infty} \psi_{i}(\xi) \psi_{j}(\xi) w(\xi) d x=\delta_{i j}\left\langle\psi_{i}^{2}\right\rangle
$$

The PCE represents any random variable $Y(\xi) \in \mathscr{L}^{2}(\Omega, F, P)$ with the $L_{2}$-convergent expansion:

$$
\begin{aligned}
& Y(\xi)=\sum_{k=0}^{N} a_{k} \psi_{k}(\xi) \\
& Y(\xi)=a_{0} \psi_{0}(\xi)+a_{1} \psi_{1}(\xi)+a_{2} \psi_{2}(\xi)+\ldots .+a_{N} \psi_{N}(\xi)
\end{aligned}
$$


where $a_{k}$ are deterministic coefficients of Polynomial Chaos, also known as the mode strength and can be calculated by a Galerkin projection operation onto the PC basis:

$$
a_{k}=\left\langle Y, \psi_{k}\right\rangle /\left\langle\psi_{k}, \psi_{k}\right\rangle
$$

The combination of deterministic mode strength $a_{k}$ and stochastic mode function $\psi_{k}$ constitutes the $k$-th mode. In practice, the denominator of Eq. 111 is known exactly, and numerical integration is used to evaluate the numerator

$$
\left\langle Y, \psi_{k}\right\rangle=\int Y(\xi) \psi_{k}(\xi) p_{\xi}(\xi) d \xi
$$

The expansion in Eq. 110 will yield exact value for $N=\infty$. However, it is not possible to execute real computations using infitine series and hence it is truncated after a finite number of terms $N$ to yield an approximate representation of $Y(\xi)$. The value of $N$ is given by:

$$
N=\left[\frac{(p+n) !}{p ! n !}\right]-1
$$

where $n$ is the number of random variables (gaussian in this case) and $p$ is the value of the highest polynomial chaos order used to represent $Y$. This approach is also referred to as a "total-order expansion" [163].

\subsubsection{Multi-Dimensional Polynomial Chaos Expansion}

For the multivariate case (where $n>1$ ), $\xi$ becomes a vector of random variables and the multi-dimensional orthogonal polynomial $\psi_{i}(\xi)$ (now denoted as $\Psi_{i}(\xi)$ ) becomes a tensor product of the polynomial bases for each component of $\xi$, when $\xi$ consists of 
IID random variables:

$$
\Psi_{k}\left(\xi_{1}, \ldots, \xi_{n}\right)=\prod_{j=1}^{n} \psi_{\alpha_{k}^{j}}\left(\xi_{j}\right)
$$

Therefore Eq. 110 can be represented as,

$$
Y(\xi)=\sum_{k=0}^{N} a_{k} \Psi_{k}(\xi)
$$

where the multi-index, $\alpha_{k}=\left[\alpha_{k}^{1}, \ldots, \alpha_{k}^{j}\right], \alpha_{k}^{j} \in \mathbb{N}$. The length of multi-index vector shall correspond to the dimensionality of the problem, i.e, the number of random variables involved. For example, Table 2 shows the multi-indexing for two-dimensional Hermite polynomials. Similarly, an example depicting the multi-index representation

\begin{tabular}{|l|l|l|}
\hline Total Order $p$ & Multivariate Polynomial $\Psi_{k}$ & Multi-index $\alpha_{k}$ \\
\hline 0 & $\Psi_{0}(\xi)=\psi_{0}\left(\xi_{1}\right) \psi_{0}\left(\xi_{2}\right)=1$ & $\alpha_{0}=[0,0]$ \\
\hline 1 & $\Psi_{1}(\xi)=\psi_{1}\left(\xi_{1}\right) \psi_{0}\left(\xi_{2}\right)=\xi_{1}$ & $\alpha_{1}=[1,0]$ \\
\hline 1 & $\Psi_{2}(\xi)=\psi_{0}\left(\xi_{1}\right) \psi_{1}\left(\xi_{2}\right)=\xi_{2}$ & $\alpha_{2}=[0,1]$ \\
\hline 2 & $\Psi_{3}(\xi)=\psi_{2}\left(\xi_{1}\right) \psi_{0}\left(\xi_{2}\right)=\xi_{1}^{2}-1$ & $\alpha_{3}=[2,0]$ \\
\hline 2 & $\Psi_{4}(\xi)=\psi_{1}\left(\xi_{1}\right) \psi_{1}\left(\xi_{2}\right)=\xi_{1} \xi_{2}$ & $\alpha_{4}=[1,1]$ \\
\hline 2 & $\Psi_{5}(\xi)=\psi_{0}\left(\xi_{1}\right) \psi_{2}\left(\xi_{2}\right)=\xi_{2}^{2}-1$ & $\alpha_{5}=[0,2]$ \\
\hline 3 & $\Psi_{6}(\xi)=\psi_{3}\left(\xi_{1}\right) \psi_{0}\left(\xi_{2}\right)=\xi_{1}^{3}-3 \xi_{1}$ & $\alpha_{6}=[3,0]$ \\
\hline 3 & $\Psi_{7}(\xi)=\psi_{2}\left(\xi_{1}\right) \psi_{1}\left(\xi_{2}\right)=\xi_{2}\left(\xi_{1}^{2}-1\right)$ & $\alpha_{7}=[2,1]$ \\
\hline 3 & $\Psi_{8}(\xi)=\psi_{1}\left(\xi_{1}\right) \psi_{2}\left(\xi_{2}\right)=\xi_{1}\left(\xi_{2}^{2}-1\right)$ & $\alpha_{8}=[1,2]$ \\
\hline 3 & $\Psi_{9}(\xi)=\psi_{0}\left(\xi_{1}\right) \psi_{3}\left(\xi_{2}\right)=\xi_{2}^{3}-3 \xi_{2}$ & $\alpha_{9}=[0,3]$ \\
\hline
\end{tabular}

Table 2: Bivariate Hermite Polynomials for 2-D and total order $p=3[2]$

of multivariate Hermite polynomials and total order $p=2$ is shown in Table 3 below. Hence, it can be observed that the problem of multi-indexing is the same as placing $p$ 


\begin{tabular}{|l|l|}
\hline Total Order $p$ & Multi-index $\alpha^{k}$ \\
\hline 0 & $\alpha_{0}=[0,0,0]$ \\
\hline 1 & $\alpha_{1}=[1,0,0]$ \\
\hline 1 & $\alpha_{2}=[0,1,0]$ \\
\hline 1 & $\alpha_{3}=[0,0,1]$ \\
\hline 2 & $\alpha_{4}=[2,0,0]$ \\
\hline 2 & $\alpha_{5}=[0,2,0]$ \\
\hline 2 & $\alpha_{6}=[0,0,2]$ \\
\hline 2 & $\alpha_{7}=[1,1,0]$ \\
\hline 2 & $\alpha_{8}=[1,0,1]$ \\
\hline 2 & $\alpha_{9}=[0,1,1]$ \\
\hline
\end{tabular}

Table 3: Multivariate Hermite Polynomials for 3D and total order $p=2$

objects into $n$ cells and listing out all probable outcomes as a vector. Also, building upon Eq. 109, the inner product of a bivariate Hermite polynomial can be written as:

$$
\left\langle\Psi_{1}, \Psi_{2}\right\rangle=\int_{\mathbb{R}^{2}} \Psi_{1}\left(\xi_{1}, \xi_{2}\right) \Psi_{2}\left(\xi_{1}, \xi_{2}\right) w\left(\xi_{1}\right) w\left(\xi_{2}\right) d \xi_{1} d \xi_{2}
$$

where, $w(\xi)=\frac{e^{\xi^{2} / 2}}{\sqrt{2 \pi}}$. For a certain $\Psi_{1}$ and $\Psi_{2}$, the above equation can be written as

$$
\begin{aligned}
\Psi_{1}\left(\xi_{n=2}\right) & =\psi_{0}\left(\xi_{1}\right) \psi_{0}\left(\xi_{2}\right) \\
\Psi_{2}\left(\xi_{n=2}\right) & =\psi_{1}\left(\xi_{1}\right) \psi_{0}\left(\xi_{2}\right) \\
\left\langle\Psi_{1}, \Psi_{2}\right\rangle & =\left(\int_{\mathbb{R}} \psi_{0}\left(\xi_{1}\right) \psi_{1}\left(\xi_{1}\right) w\left(\xi_{1}\right) d \xi_{1}\right)\left(\int_{\mathbb{R}} \psi_{0}\left(\xi_{2}\right)^{2} w\left(\xi_{2}\right) d \xi_{2}\right)
\end{aligned}
$$


In a general form, we can represent the orthogonality of multidimensional inner product as [164]

$$
\begin{aligned}
\left\langle\Psi_{i} \Psi_{j}\right\rangle & =\int \ldots \int \Psi_{i}(\xi) \Psi_{j}(\xi) w\left(\xi_{1}\right) w\left(\xi_{2}\right) \ldots w\left(\xi_{n}\right) d \xi_{1} d \xi_{2} \ldots d \xi_{n} \\
& =\prod_{k=1}^{n}\left\langle\psi_{\alpha_{k}^{i}}\left(\xi_{k}\right) \psi_{\alpha_{k}^{j}}\left(\xi_{k}\right)\right\rangle=\delta_{i j}\left\langle\Psi_{i}^{2}\right\rangle
\end{aligned}
$$

\subsubsection{Methods for non-intrusive implementation of PCE}

Implementation of PCE requires the estimation of polynomial coefficients $a_{k}$ in Eq. 111. Also known as the black-box technique, non-intrusive polynomial chaos (NIPC) primarily aims at obtaining the polynomial coefficients without modifying the deterministic code, i.e., the deterministic model is considered as a "black-box" and the polynomial coefficients are determined using formulae based on deterministic outputs. The sampling points for input needed to run the deterministic code are based on the chosen non-intrusive method. Non-intrusive methods can be categorised as projection methods (sampling-based or quadrature-based) [66, 165], regression methods [166], probabilistic collocation methods [167] and gradient-based methods [168].

\section{Spectral Projection}

In this study, we focus on projection methods. There are two approaches to projection methods, i.e, random sampling and quadrature. The main strategy in random sampling based method is to compute the numerator in Eq. 111 for a number of samples and average it to provide an estimate of the inner product. For multivariate polynomials, the denominator $\left\langle\Psi_{k}^{2}(\xi)\right\rangle$ can be produced analytically. Evidently, the accuracy of this technique is dependent on the number of samples used to approximate the expectation but there are considerable departures from, and advantages over direct MCS. We'll discuss these advantages in the subsequent section.Consider 
the following equation,

$$
F(\xi)=\int_{a}^{b} f(\xi) p(\xi) d \xi \approx \sum_{i}^{n} f\left(\xi_{i}\right) w_{i}
$$

Alternatively to random sampling, a numerical quadrature rule can be used to evaluate the integral defining the inner product by selecting $w_{i}$ such that the approximation is exact for a set of $n$ basis elements. In Eq. 119, $p(\xi)$ is the weight function and $w_{i}$ is the quadrature weight at the quadrature node $\xi_{j}$. A Monte Carlo simulation will set $w_{i}=\frac{1}{n}$ and choose $\xi_{i}$ randomly. For 1-D problems, the Gaussian quadrature approach uses nodes that are the roots of optimal orthogonal polynomials for the given input probability distribution. For example, a Gauss Hermite quadrature rule will be an obvious choice for a $N(0,1)$ random variable. Multidimensional quadrature can be constructed from 1-D quadratures using tensor product method. However, tensor products of quadrature rules demand even more runs of the deterministic solver than the MCS. Despite the existence and efficiency of sparse quadrature rules, quadrature is not the best choice when the random input is high dimensional [169].

For the problem of seismic wave propagation, a realistic scenario would entail consideration of many layers of soil strata with different properties. This will lead to many random parameters and hence numerical quadrature will become very computationally demanding due to the number of required quadrature points. We chose the sampling based method because it is not restricted by this limitation.

Given below are the MATLAB codes for one-dimensional non-intrusive spectral projection (NISP) expansion of a function $Y=X^{2}$, implementing both quadrature and sampling based methods respectively. Both the codes run for highest polynomial order of 2 and for a standard normal random variable $\xi$, thereby approximating the given function with coefficients $a_{1}=1 ; a_{2}=0 ; a_{3}=1$ of PCE. These values are 
correct because it implies that

$$
\begin{aligned}
Y & =X^{2}(\xi) \\
& =(1)(1)+(0)[X(\xi)]+(1)\left[X^{2}(\xi)-1\right] \\
& =X^{2}(\xi)
\end{aligned}
$$

$1 \%$ Calculation of PCE coefficients based on Gauss hermite quadrature

$2 \mathrm{n}=10 ; \%$ Number of quadrature points

$3 \mathrm{nV}=1: \mathrm{n}-1$

4 BetaSqrt $=\operatorname{sqrt}(\mathrm{nV})$;

$5 \mathrm{~J}=\operatorname{diag}($ BetaSqrt, 1) $+\operatorname{diag}($ BetaSqrt, -1$) ;$

$6[\mathrm{~V}, \mathrm{D}]=\operatorname{eig}(\mathrm{J})$;

$7[\mathrm{x}$, ind $]=\operatorname{sort}(\operatorname{diag}(\mathrm{D}))$;

$8 \%$ Vector ' $X$ ' contains the location of quadrature nodes

9 Vtop $=\mathrm{V}(1,:)$;

10 Vtop $=\operatorname{Vtop}($ ind $)$;

$11 \quad \mathrm{w}=\operatorname{sqrt}(2 * \mathrm{pi}) *$ Vtop. ${ }^{\wedge} 2$;

$12 \%$ Vector ' $w$ ' contains the weight of quadrature nodes

$13 \mathrm{mu}=0$;

14 sigma $=1$;

$15 \mathrm{xx}=\mathrm{mu}+\operatorname{sigma} * \mathrm{x} ;$

$16 \mathrm{y}=\mathrm{xx} \cdot \leadsto 2 ; \%$ Main function

$17 \%$ Integrating the inner products

18 Den_HeO $=0$; Den_He1 $=0$; Den_He2 $=0$;

19 Num_HeO $=0$; Num_He1 $=0 ; \quad$ Num_He2 $=0$;

$20 \%$ Den_He_n $=\operatorname{sum}\left(\right.$ w.* $\left.*\left(\left(\exp \left(.5 *\left(\mathrm{x} \cdot{ }^{\wedge} 2\right)\right) /(\operatorname{sqrt}(2 * \mathrm{pi}))\right) \cdot *(\mathrm{x}) \cdot{ }^{\wedge} 2\right)\right)$

21 for $i=1: n$

$22 \quad$ Den_HeO $=$ Den_HeO $+(1 /(\operatorname{sqrt}(2 *$ pi $))) *($ w $(i) * 1) ;$ 


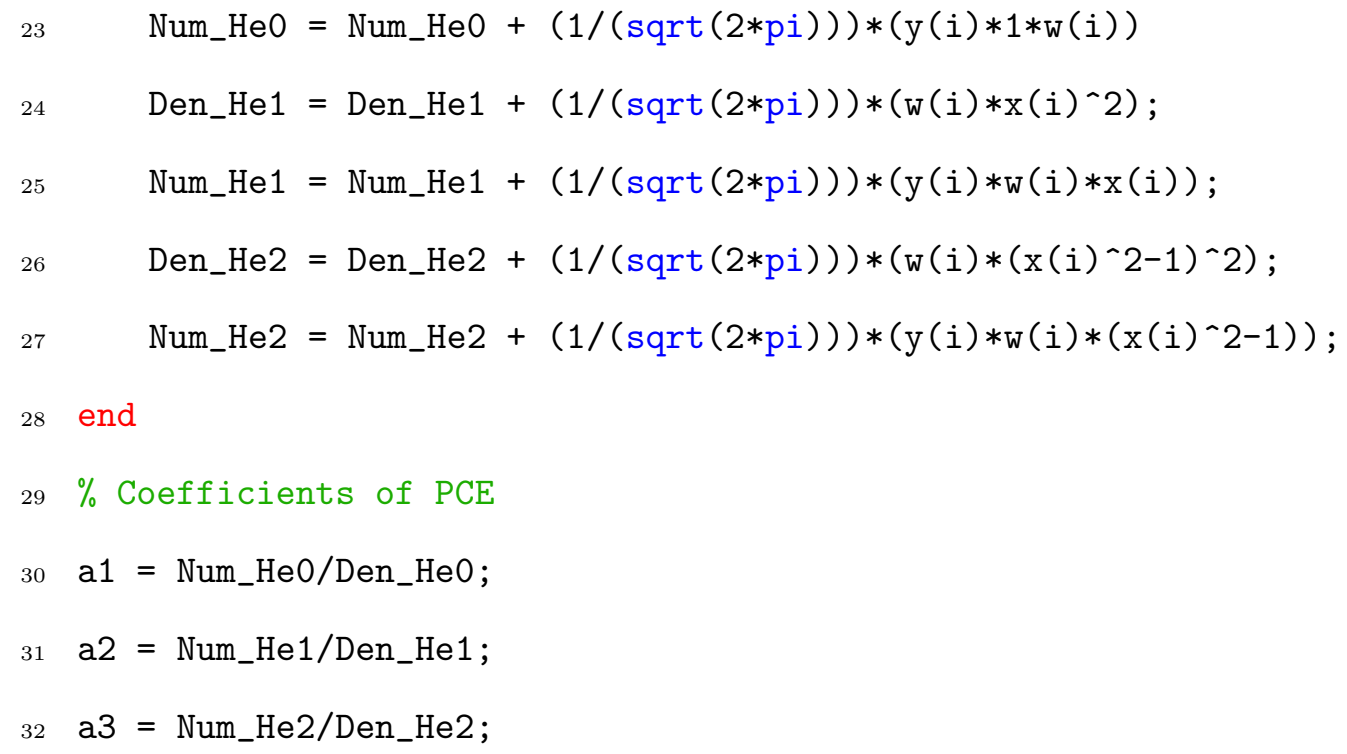




\section{PCE for vector valued response}

The examples provided in the previous section represent a scalar output function (having finite variance) with a set of scalar PCE coefficients. However, we solve a wave equation having vector-valued solution or output. Therefore, we need to extend Eq. 115 to accommodate vector-valued response $Y=\left(Y^{1}, Y^{2}, \ldots, Y^{n}\right)$. This can be achieved by individually expanding each of the outputs independently on the same truncated basis [170]:

$$
Y^{i}(\xi)=\sum a_{k}^{i} \Psi_{k}(\xi), \quad i=1, \ldots, n
$$

For each component of $Y$, the mean value is given by the first coefficient $a_{0}$. The concept of PCE for vector-valued outputs can be understood better through the following MATLAB code which plots the PCE coefficients for the case of a single random variable on a 1-D mesh for 2 nd order expansion. A non-linear function has been used such that the coefficients for Hermite polynomials are already known.

$$
Y=(1)(1)+(\xi)[\cos (4 \pi x / l)]+\left(\xi^{2}-1\right)[\cos (8 \pi x / l)]
$$

where $l=10$ is the length of the $1-\mathrm{D}$ mesh and $\xi N(0,1)$. The analytical solution of the known coefficients are plotted with the respective obtained PCE coefficients in Fig. 23. The total number of samples used is five hundred, and a good match is observed between the analytical solution and the observed 1st and 2nd order PCE coefficients. This solution may suffer from sampling error. However, an acceptable consistency in solution is obtained for different sets of samples.

\footnotetext{
$1 \%$ Case 1: One Random variable, 1D mesh, 2nd order

$2 x=0: 0.1: 10 ; \quad \%$ Array of Nodes on the mesh (length)
} 


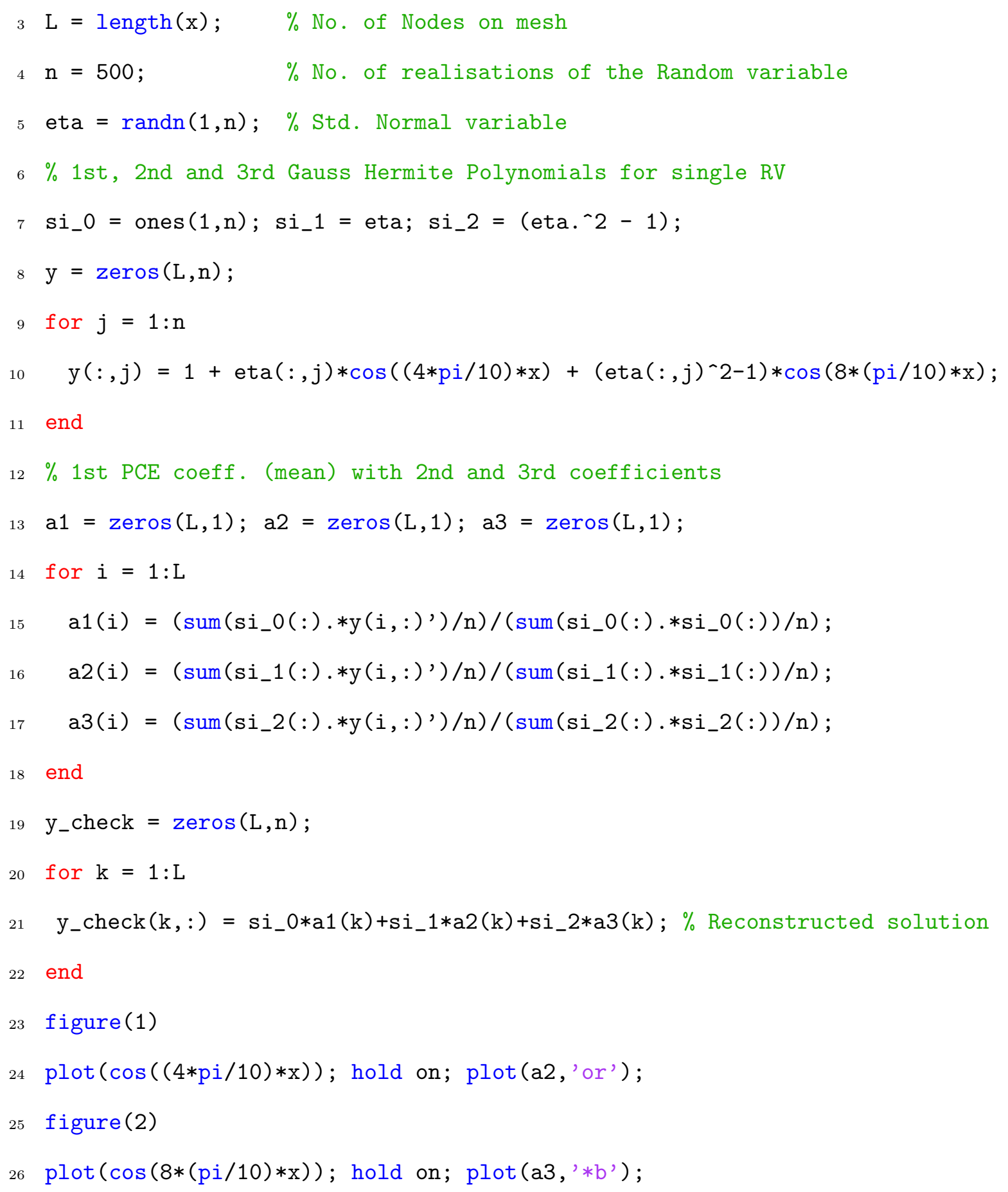

The same code was modified for the case of 2 random variables with $3 r d$ order expansion, on a 1-D mesh for the following function: 


$$
\begin{aligned}
Y= & (1)(1)+\left(\xi_{1}\right)[\cos (4 \pi x / l)]+\left(\xi_{2}\right)[\cos (8 \pi x / l)]+\left(\xi_{1}^{2}-1\right)[\cos (4 \pi x / l)] \\
& +\left(\xi_{1} \xi_{2}\right)[\cos (16 \pi x / l)]+\left(\xi_{2}^{2}-1\right)[\cos (8 \pi x / l)]+\left(\xi_{1}^{3}-3 \xi_{1}\right)[\cos (4 \pi x / l)] \\
& +\left[\xi_{2}\left(\xi_{1}^{2}-1\right)\right][\cos (8 \pi x / l)]+\left[\xi_{1}\left(\xi_{2}^{2}-1\right)\right][\cos (16 \pi x / l)]+\left(\xi_{2}^{3}-3 \xi_{2}\right)[\cos (32 \pi x / l)]
\end{aligned}
$$

The higher order PCE coefficients for Eq. 122 have been plotted in Fig. 24, 25 and 26. For five hundred samples, a decent match is observed among estimated coefficients and their analytical values. As discussed previously, a PCE for infinite terms will give the exact solution. However, it has to be truncated at some point for computational expedience. Usually, expansion upto $3 r d$ order is considered to provide a fairly accurate approximation of the exact solution. Now, we extend the 1-D problem in Eq. 122 to a 2-D problem. A square mesh with sides of length $l$ is considered for the following 2-D function:

$$
\begin{aligned}
Y=(1)(1)+\left(\xi_{1}\right)[\cos (4 \pi x / l) \cos (4 \pi y / l)]+ & \left(\xi_{2}\right)[\cos (8 \pi x / l) \cos (8 \pi y / l)] \\
+\left(\xi_{1}^{2}-1\right)[\cos (4 \pi x / l) \cos (4 \pi y / l)]+ & \left(\xi_{1} \xi_{2}\right)[\cos (16 \pi x / l) \cos (16 \pi y / l)] \\
& +\left(\xi_{2}^{2}-1\right)[\cos (8 \pi x / l) \cos (8 \pi y / l)]
\end{aligned}
$$

Some 1 st and 2 nd order PCE coefficients for the above function have been shown in Fig. 27 and 28. It can be observed that both the shape and the contour plots are equally important to observe the behaviour of PCE coefficients. In Fig. 27b where only the shape has been plotted, it is difficult to analyse the values at peaks, and a contour plot shown in Fig. 28a may prove convenient. At the same time, while the contour plots for Fig. 27c and Fig. 28a may appear reasonably similar, it is also important to view their shapes to form a conclusive opinion. A total of five hundred samples were used to generate these plots and even though the results can 


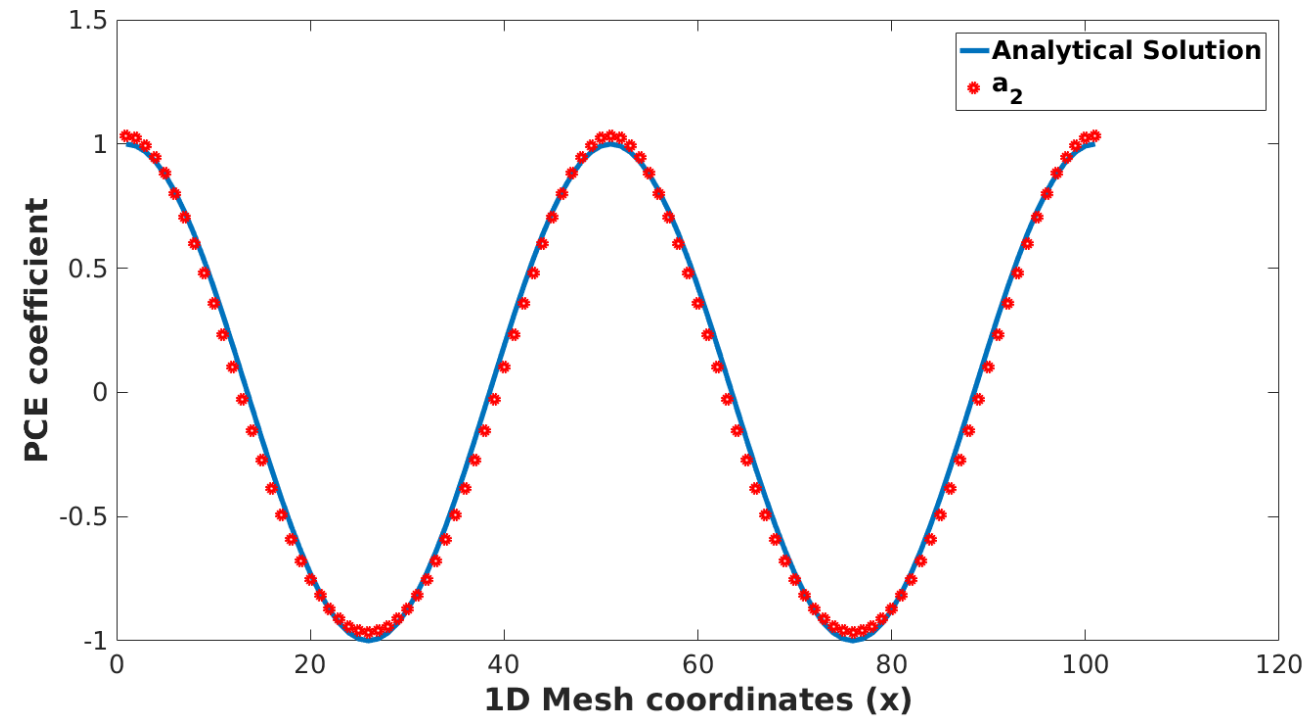

(a) 2nd PCE coefficient

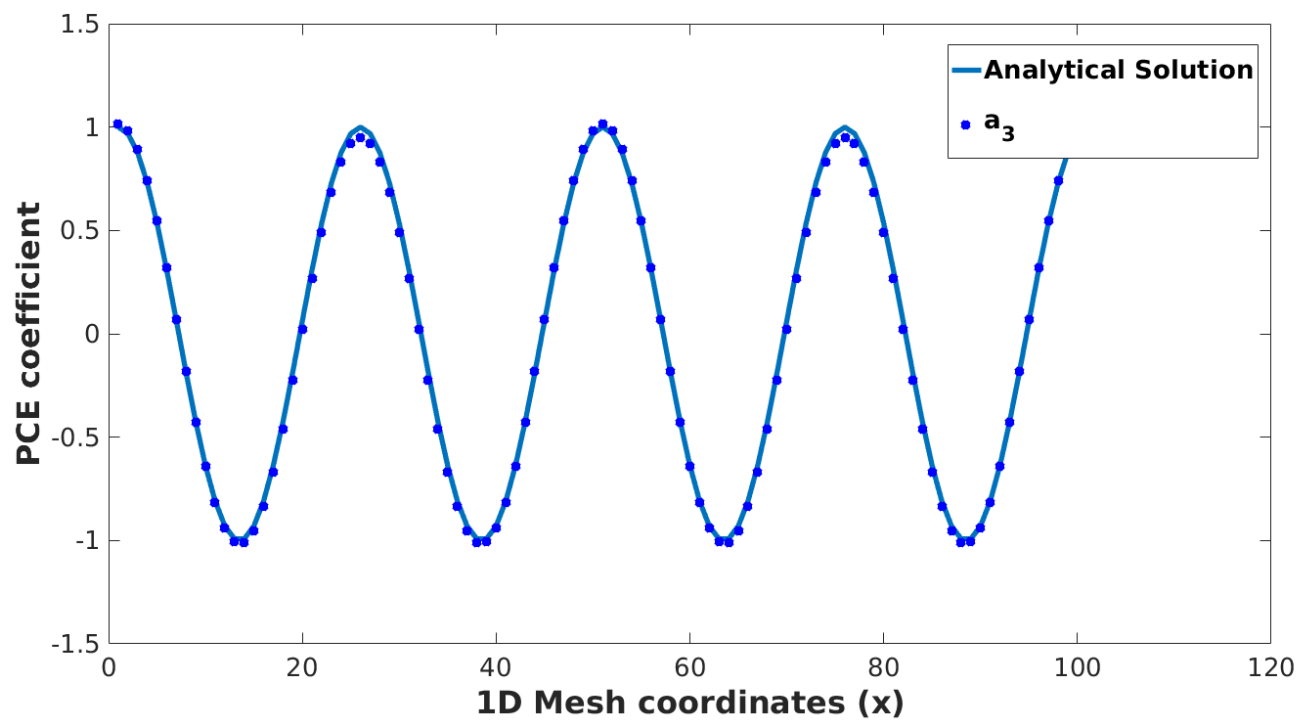

(b) 3rd PCE coefficient

Figure 23: PCE coefficients for the case of a single random variable with 2 nd order expansion, on a 1D mesh 
undoubtedly be better with more samples, there seem to be a satisfactory trade off between accuracy of the solution and required computational effort.

\subsubsection{Why use PCE?}

The differences between intrusive and non-intrusive PCE were discussed in Chapter 2, and the use of a non-intrusive method was advocated. Efficiency and flexibility of PCE were also established in comparison to other uncertainty quantification methods like perturbation and Monte Carlo approach $[57,59,60]$. However, the use of a sampling based spectral projection method in this study gives rise to a common question, i.e., how is this partially sampling based technique free from the shortcomings of traditional Monte Carlo method?

The answer lies in the number of samples required to achieve reliable accuracy using both the methods. The Monte Carlo method requires a huge number of samples to give reliable results while PCE is an efficient alternative to the overwhelmingly low convergence rate of Monte Carlo. Exponential convergence rates can be obtained using PCE with an optimal basis [163] because NISP achieves spectral convergence with only a few runs of PDE solver, while Monte Carlo simulation requires thousands of PDE solutions for appropriate convergence [171]. The purpose of PCE is to parametrise the solution to regularise the stochastic problem. Also, moments are much easier to calculate using PCE than in the Monte Carlo method.

Using this method, we can plot the pdf at any given point by first generating the PCE coefficients from a set of few sufficient number of samples, and then generating many more samples using the estimated PCE based solution as the surrogate. In this way, the governing equations don't need to be solved repetitively as required for direct Monte Carlo method. Therefore, this method allows us to get more number of samples consistent with the information that we have, without much computational 
effort. It also allows us to observe the difference between Gaussian and non-Gaussian effect on our solution through the use of higher order coefficients. The solution reconstructed by using PCE coefficients of first order Hermite polynomials will only show the Gaussian effects, while the non-Gaussian effects can be observed when coefficients of quadratic and cubic Hermite polynomials are added to reconstruct the solution.

It is reasonable to state that PCE is a relatively cheap way to get a surrogate model for the randomness in the system. In addition to all of the above listed advantages, this method also conveniently blends into the framework of other uncertainty quantification techniques. For instance, one can also perform Global Sensitivity Analysis using non-intrusive PCE [172].

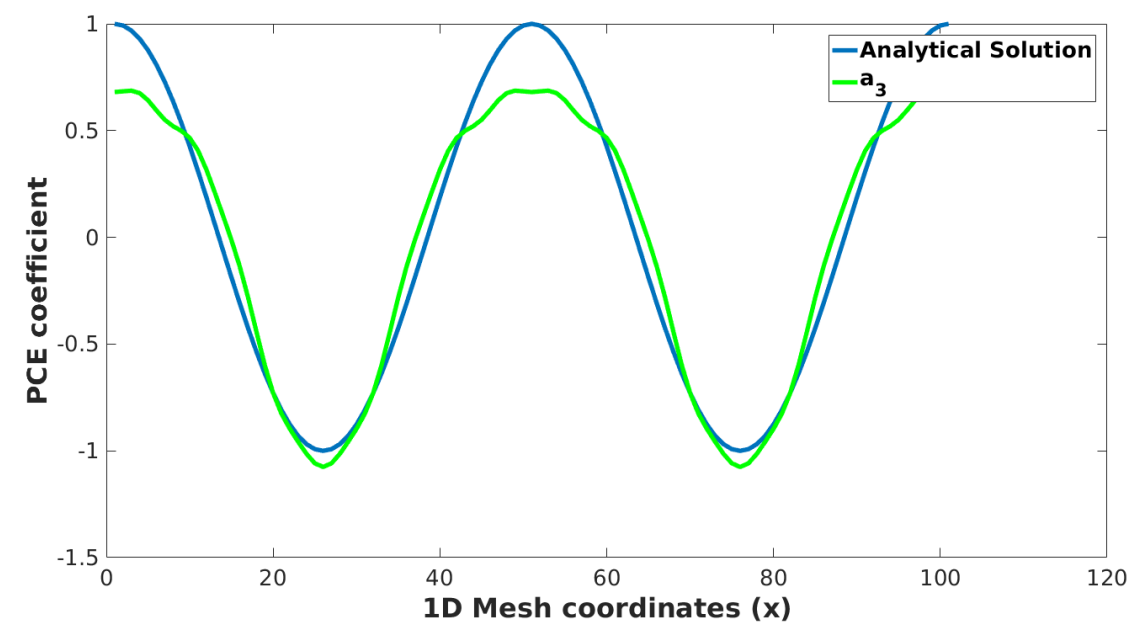

Figure 24: 4th PCE coefficient $a_{3}$ (sampling-based) for the case of two random variables with $3 r d$ order expansion, on a $1 \mathrm{D}$ mesh 


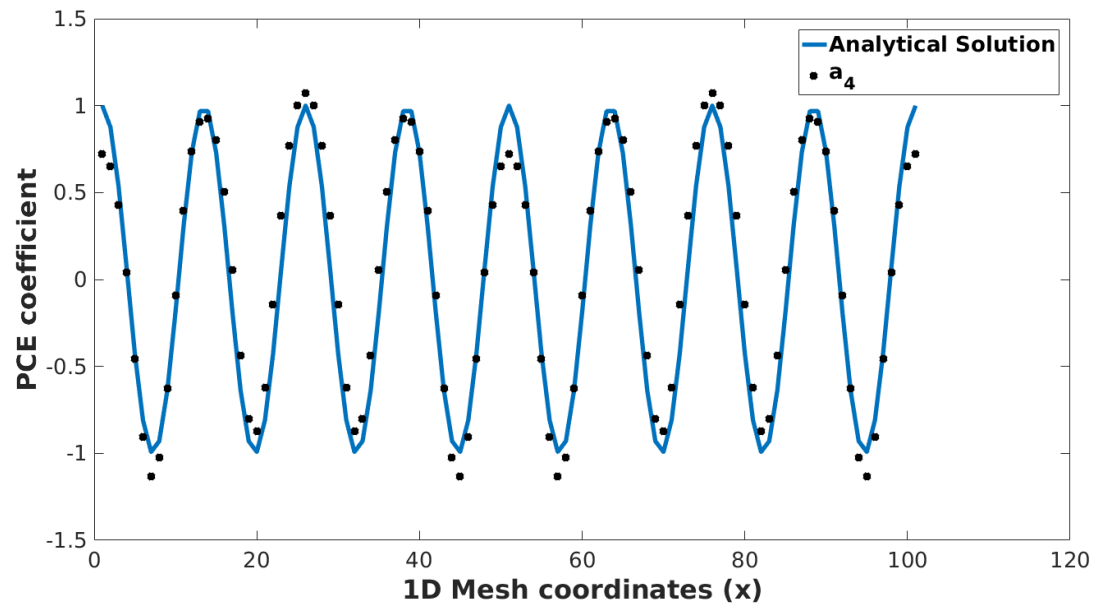

(a) 5th PCE coefficient

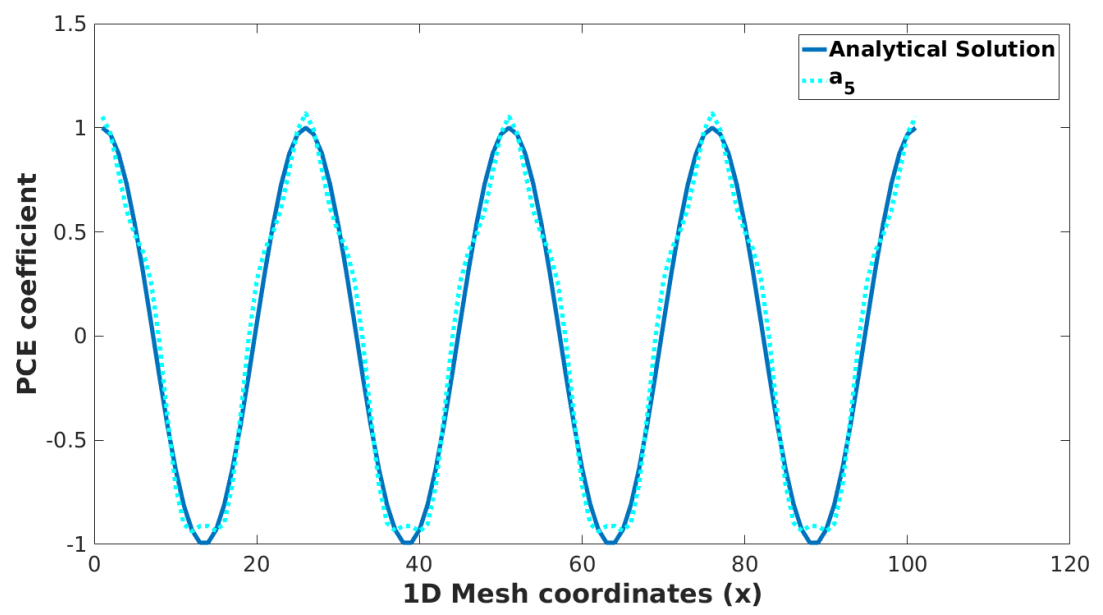

(b) 6th PCE coefficient

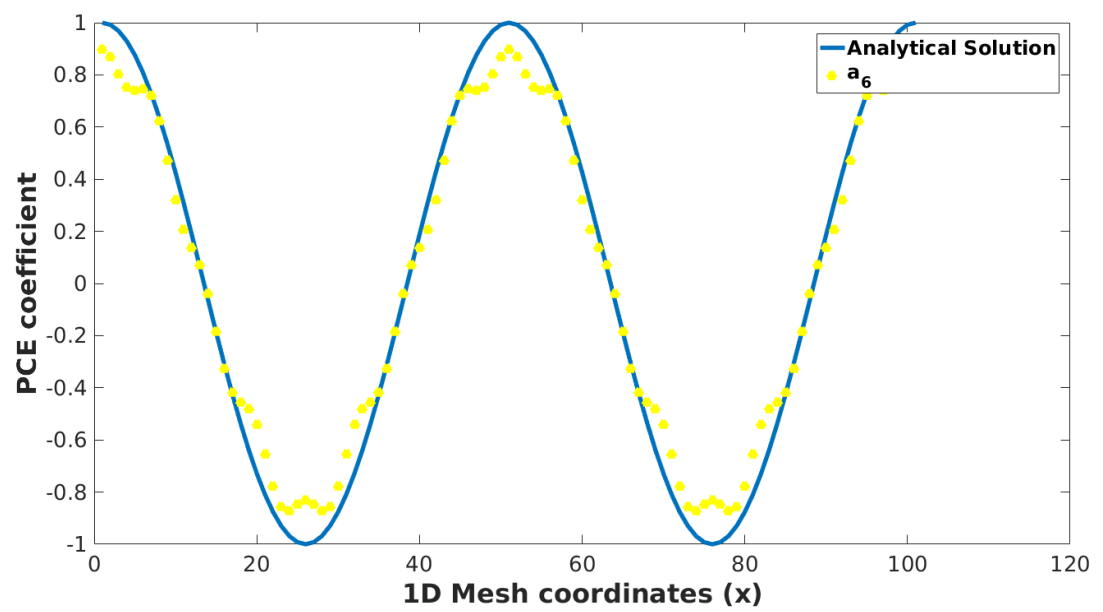

(c) $7 t h$ PCE coefficient

Figure 25: PCE coefficients $a_{4}, a_{5}, a_{6}$ (sampling-based) for the case of two random variables with $3 r d$ order expansion, on a $1 \mathrm{D}$ mesh 


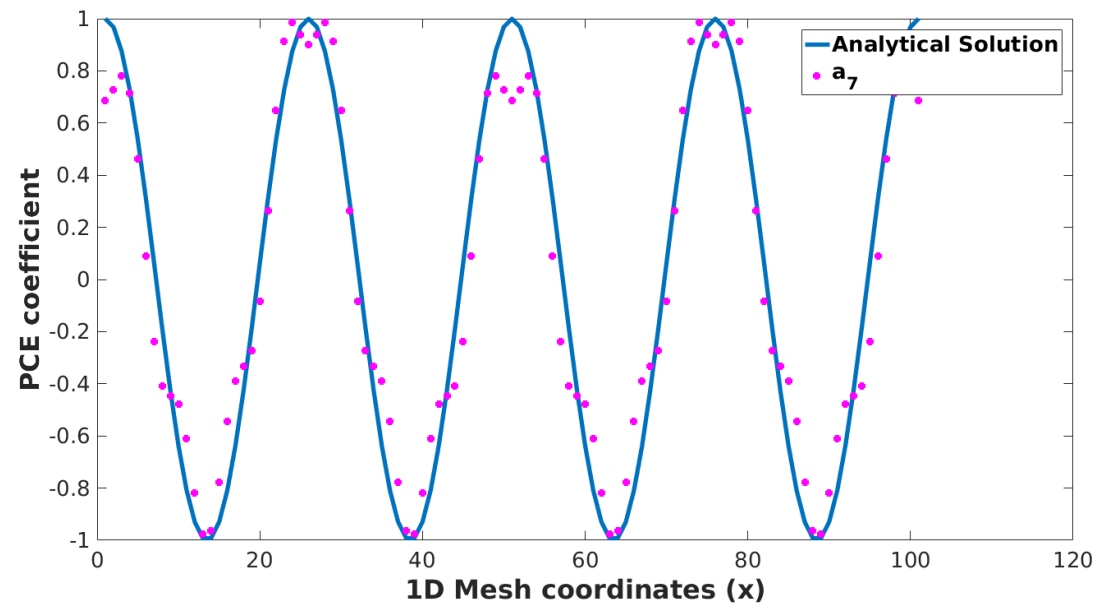

(a) 8th PCE coefficient

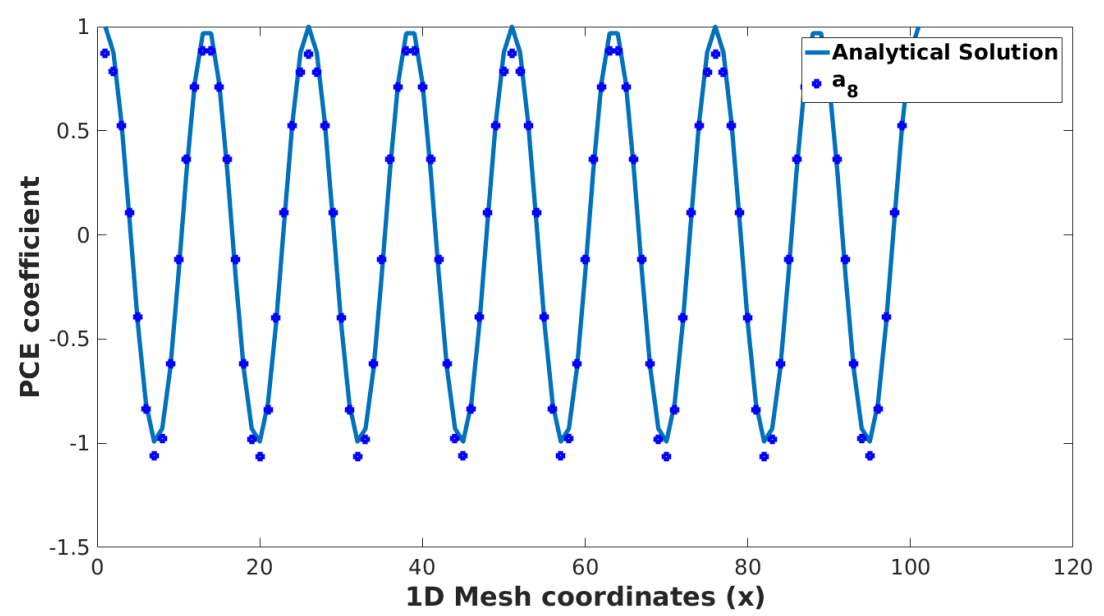

(b) 9 th PCE coefficient

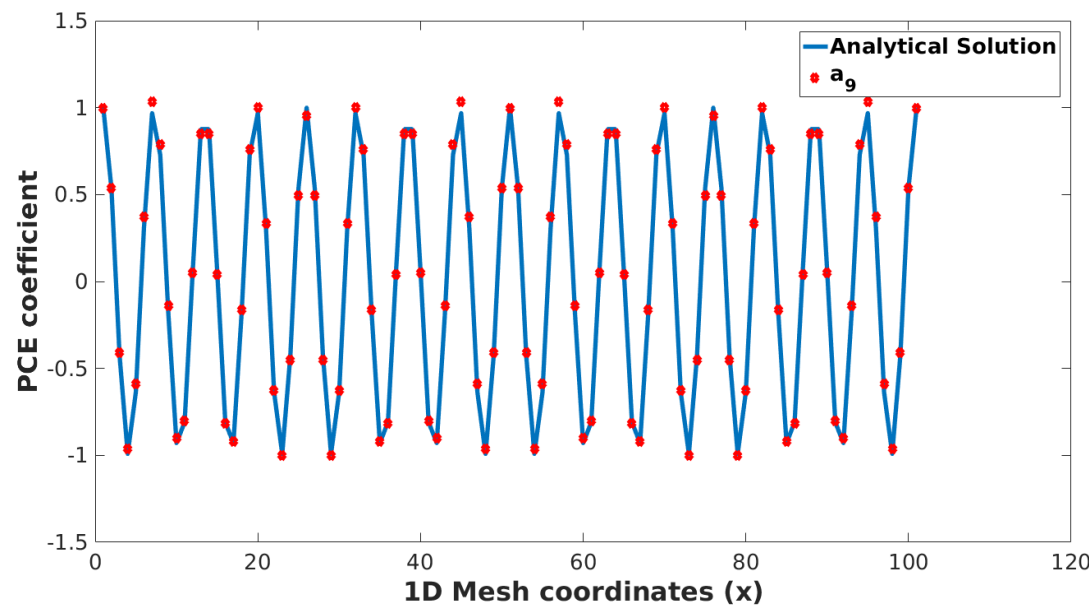

(c) $10 t h$ PCE coefficient

Figure 26: PCE coefficients $a_{7}, a_{8}, a_{9}$ (sampling-based) for the case of two random variables with $3 r d$ order expansion, on a $1 \mathrm{D}$ mesh 


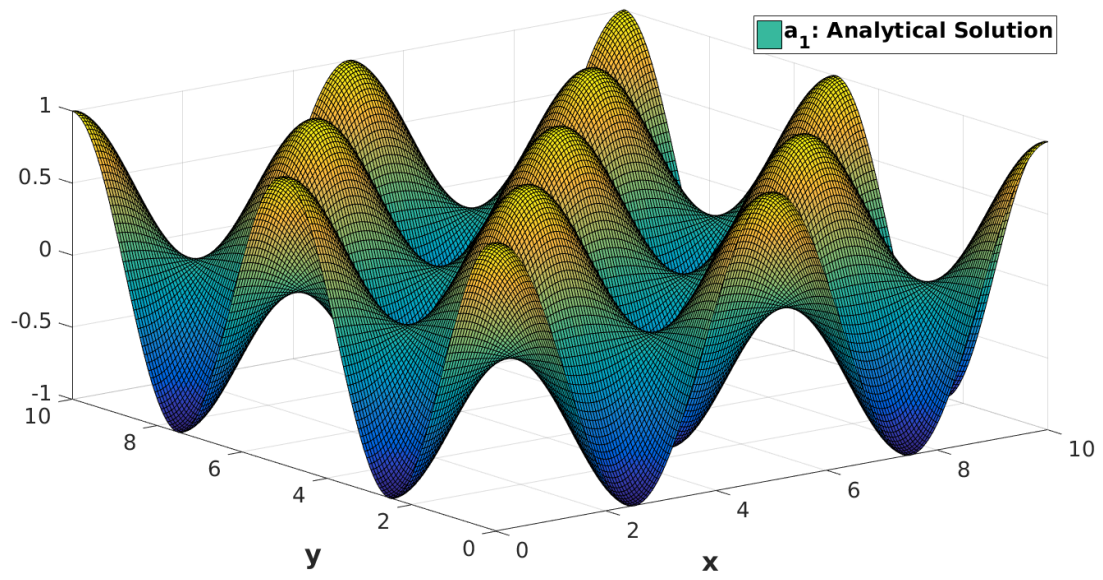

(a) 2nd PCE coefficient (analytical)

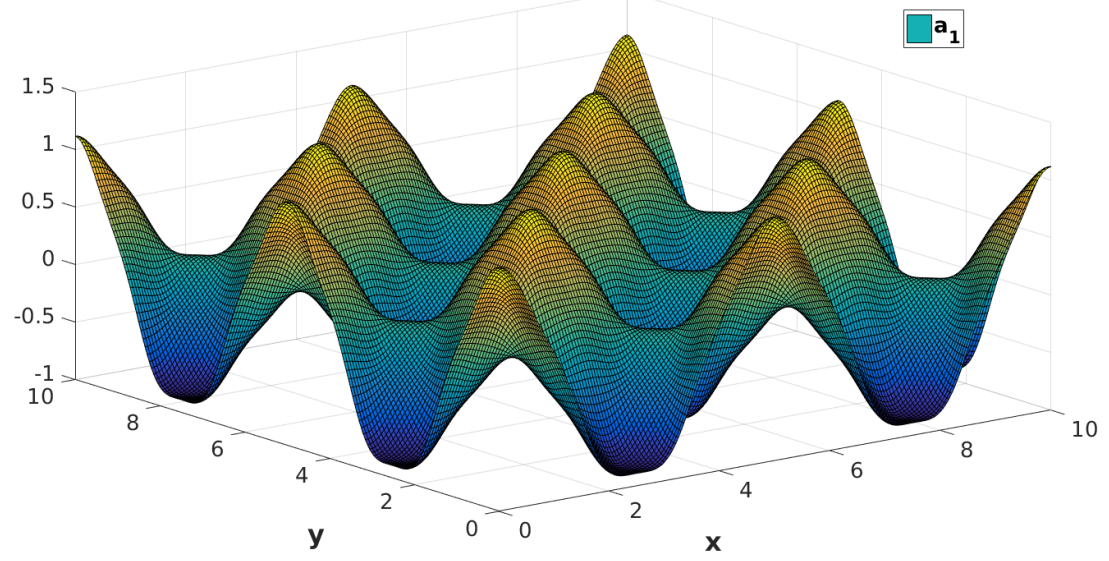

(b) 2nd PCE coefficient

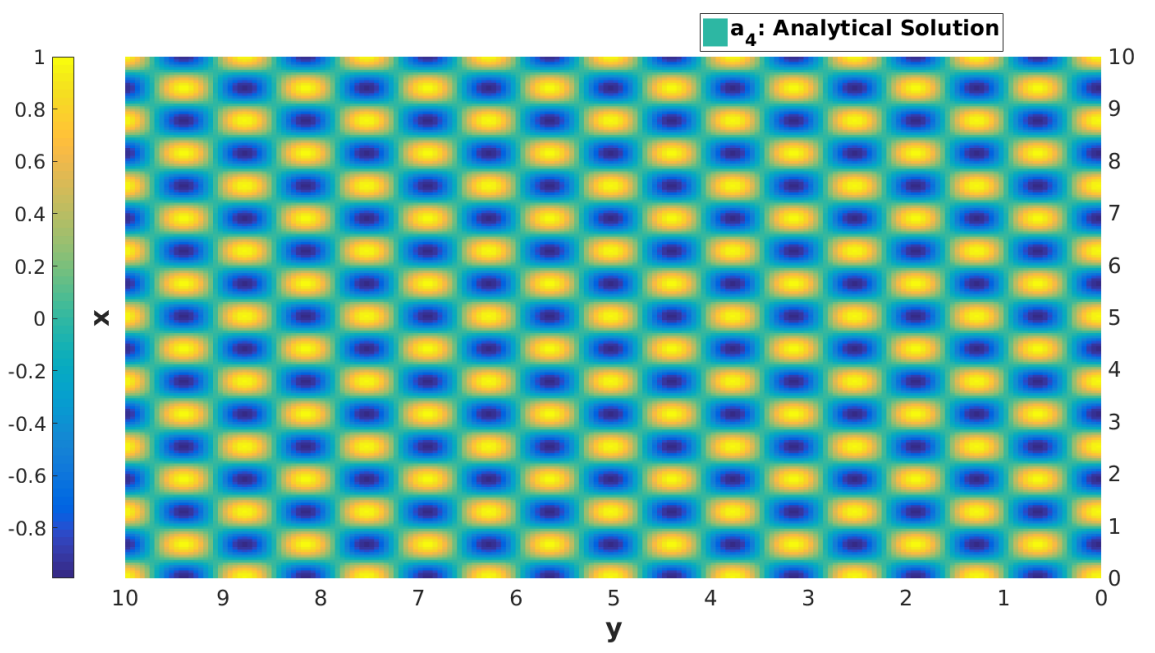

(c) 5th PCE coefficient (analytical)

Figure 27: PCE coefficients $a_{1}, a_{4}$ (sampling-based) for the case of two random variables with 2 nd order expansion, on a $2 \mathrm{D}$ mesh 


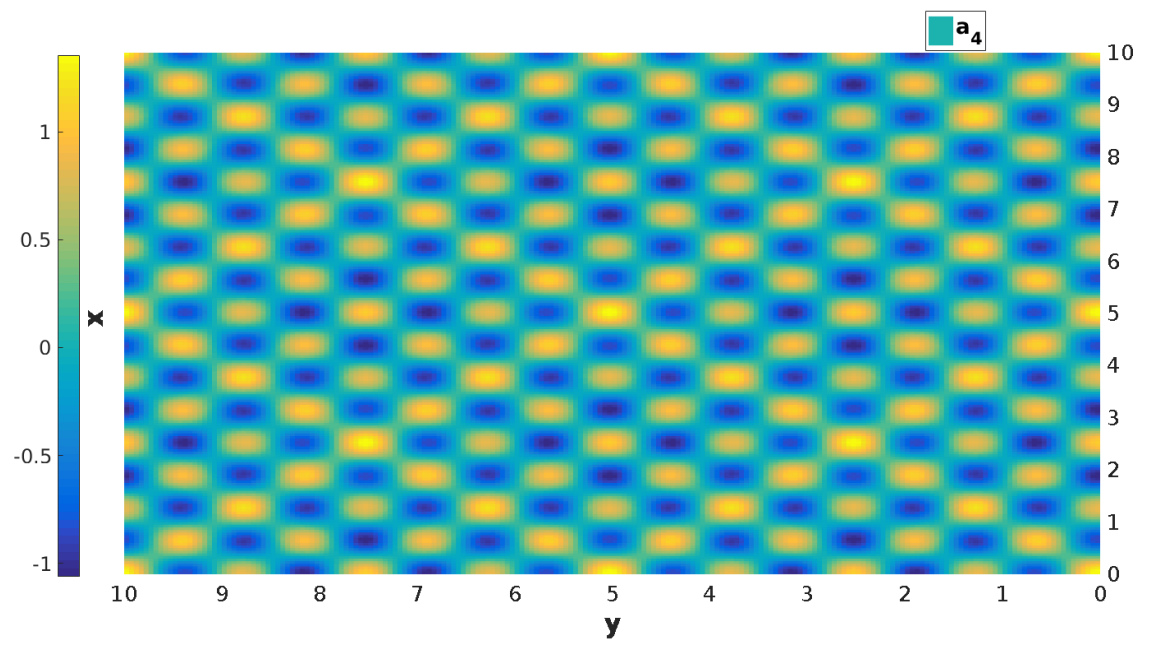

(a) 5th PCE coefficient

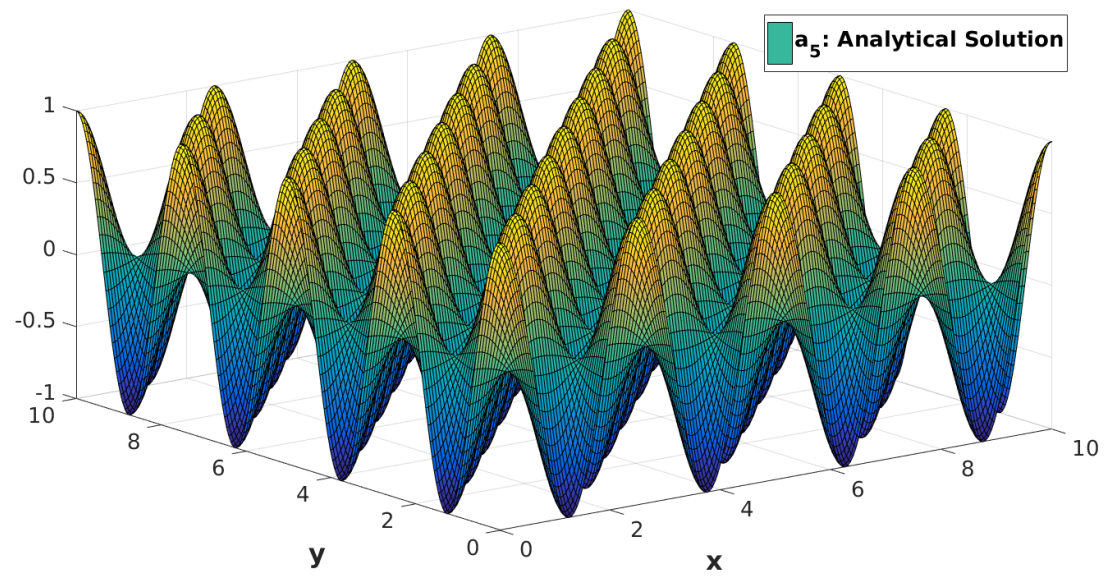

(b) 6th PCE coefficient (analytical)

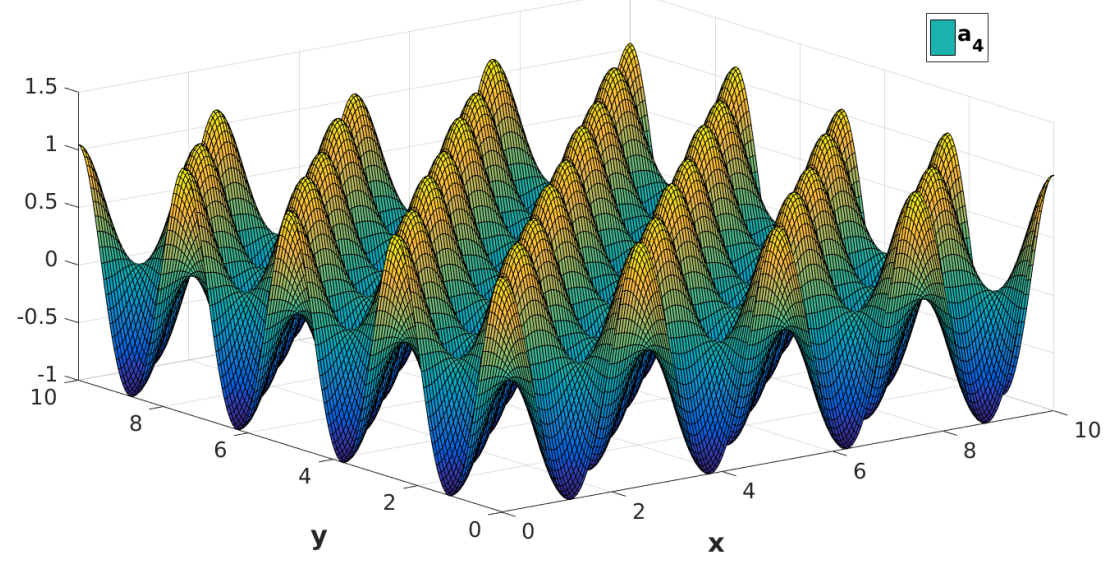

(c) 6th PCE coefficient

Figure 28: PCE coefficients $a_{4}, a_{5}$ (sampling-based) for the case of two random variables with $2 n d$ order expansion, on a $2 \mathrm{D}$ mesh 


\subsection{Application of non-intrusive PCE to wave propagation}

Given the many applications of wave propagation problem, it is worthwhile to investigate the effect of variability in its solution. In a deterministic simulation, the statistical variability of system properties are ignored from the simulation results by assuming the nominal values of system parameters. For a material like soil, where very high coefficients of variation $(\mathrm{COVs})$ may exist $(10-40 \%)$ [173], this uncertainty is compensated for, by the use of a high safety factor. This approach is not economical and in some cases, may even be unsafe [44].

Therefore, we illustrate the application of a non-intrusive spectral projection (NISP) method, for three numerical examples with parametric uncertainties, namely (a) a linear elastic 2D homogeneous soil medium, (b) a linear elastic 2D layered piecewise homogeneous soil medium, (c) a non-linear elasto-plastic 2D homogeneous soil medium. To accomplish this, we begin by identifying the parameters whose variabilities have the most effect on the response of the solution [50]. Thereafter, for all the important physical parameters, we chose a mean value consistent with the values observed in Ottawa valley for a slightly over consolidated soil, and assign a COV to each of them. To implement a sampling based NISP method, we simulate 500 samples for each case and then approximate the solution using polynomial chaos expansion. All

the simulations for Case 1 and 3 have been performed on a square mesh of size $[1,1]$ $\mathrm{km}^{2}$ discretized into approximately 170,000 triangular cells. The source is modelled as a Gaussian pulse in space $f=\left(f_{x}, f_{y}\right)$ given by Eq. 44 , at the centre of the mesh. 


\subsubsection{Case 1: Linear elastic wave propagation in homoge- neous media}

We now consider the case of linear elastic waves propagating in an isotropic homogeneous soil medium. For this case, two physical input parameters, i.e., the Young's Modulus $E$ and the density $\rho$ have been considered as random variables. Truncated Gaussian distributions are assumed for $E$ and $\rho$ such that all values remain positive. The assumed mean values and COVs for the input parameters used in the simulation are given in the Table 4 . The chosen mean values correspond to a shear modulus value of $G=25 \mathrm{MPa}$ and a shear wave velocity $\left(V_{s}=\sqrt{G / \rho}\right)$ of $120 \mathrm{~m} / \mathrm{s}$. A comparison of the deterministic and the mean solution from PCE has been shown in Fig. 29-31. It can be noted that the estimated mean wave profile is considerably different from the deterministic response. The resulting magnitude contours for mean particle displacement show a more subtle (low magnitude) and spatially distributed response compared to the respective deterministic outputs. There is a clear lack of the pronounced sharp variations that are present in the deterministic simulations. The difference between the expected and the deterministic solution will always be dependent on the statistical distribution of the input parameters (in this case Gaussian) and their COV.

Thereafter, some PCE coefficients with increasing order have been plotted in Fig. 32-36. The PCE coefficient shown in Fig. 32 is one of the 1st order coefficients which represent the Gaussian contribution to the solution, while the $2 n d$ and $3 r d$ order coefficients shown in Fig. 33-36 represent the non-Gaussian contribution. The PCE coefficients are interesting to observe because they are an integral part of the parametrised solution and can be used to cheaply reconstruct innumerable potential solutions based on newly generated random samples. The glyph plots shown alongside 
the magnitude plots are used to represent the direction of solution vector for multidimensional simulations. Glyphs are polygonal objects such as a cone or an arrow which display displacement vectors with components in $x, y, z$ through the direction and length of the arrow. In Fig. 37, the standard deviation has been plotted for three time steps. The prominent scatter of the output provides solid proof that huge uncertainty is involved in the prediction of ground motion. This uncertainty can be attributed to the large COV used for the input physical parameters. It can also be observed that this uncertainty reduces as the time evolves.

\begin{tabular}{|l|l|l|}
\hline Parameter & Mean $\mu$ & COV (\%) \\
\hline$E$ & $64 \mathrm{MPa}$ & 40 \\
\hline$\nu$ & 0.3 & - \\
\hline$\rho$ & $1700 \mathrm{~kg} / \mathrm{m}^{3}$ & 10 \\
\hline$\eta$ & $5 \%$ & - \\
\hline
\end{tabular}

Table 4: Values of mean and COV for parameters of the linear elastic model 


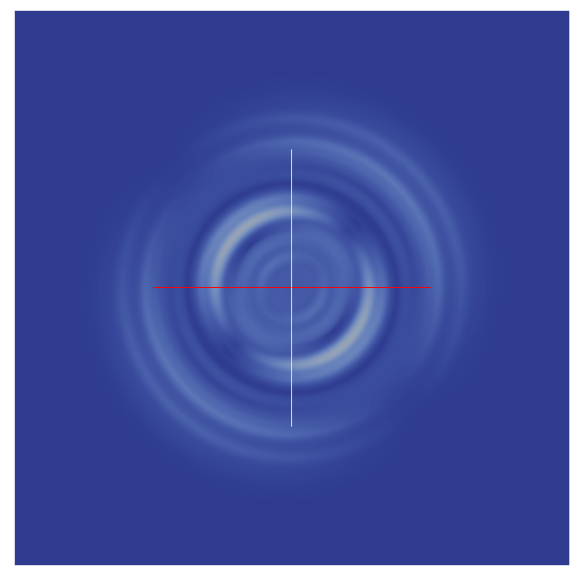

(a) Mean: Magnitude

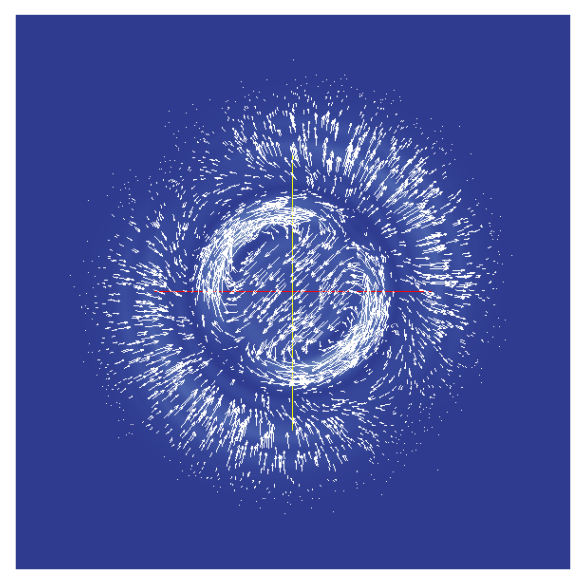

(b) Mean: Glyph

$\mathrm{km}$

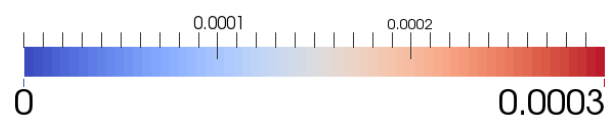

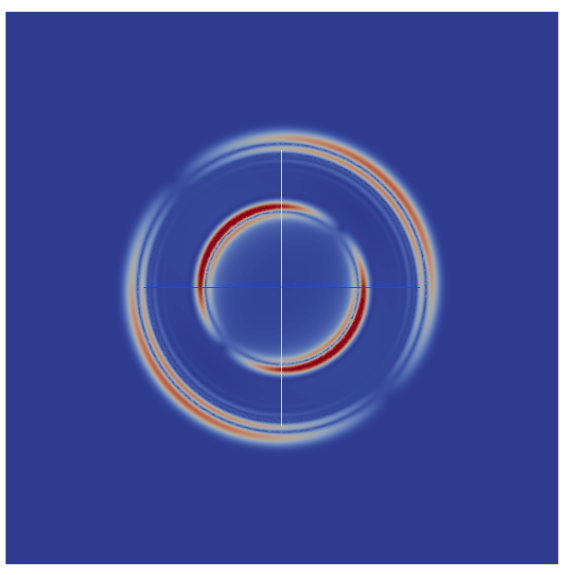

(c) Deterministic: Magnitude

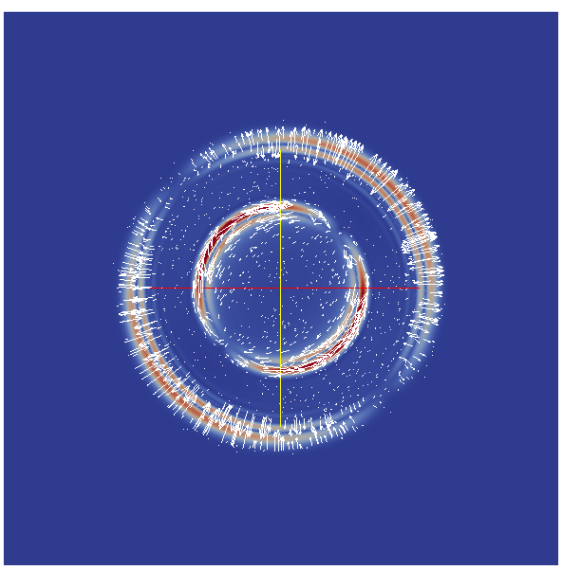

(d) Deterministic: Glyph

$\mathrm{km}$

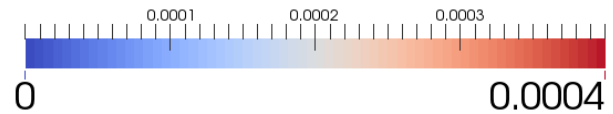

Figure 29: Magnitude of waves propagating in a homogeneous elastic soil medium: mean $\left(a_{0}\right)$ and deterministic output at $t=1.2 \mathrm{~s}$ 


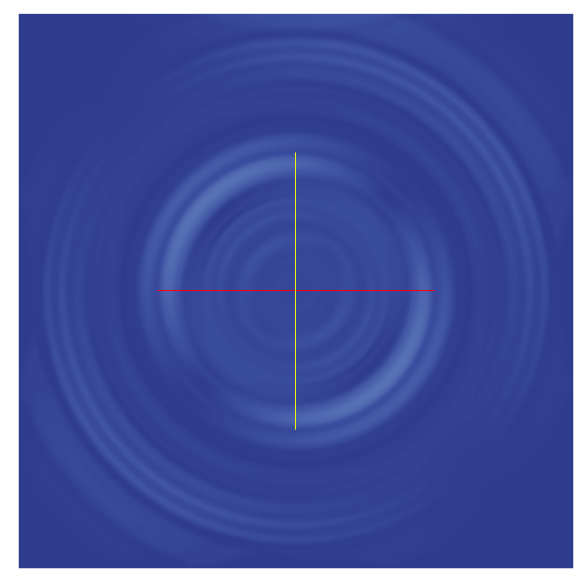

(a) Mean: Magnitude

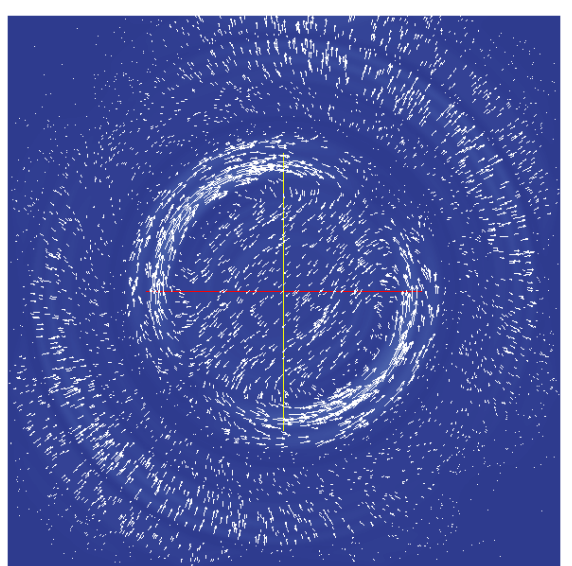

(b) Mean: Glyph

$\mathrm{km}$

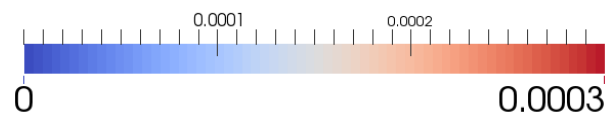

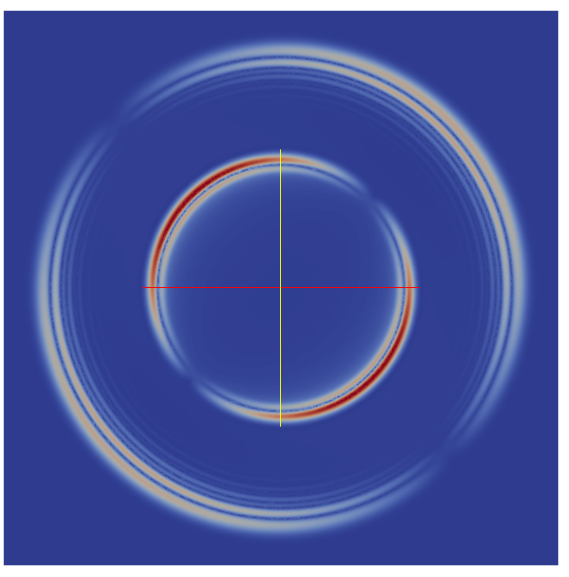

(c) Deterministic: Magnitude

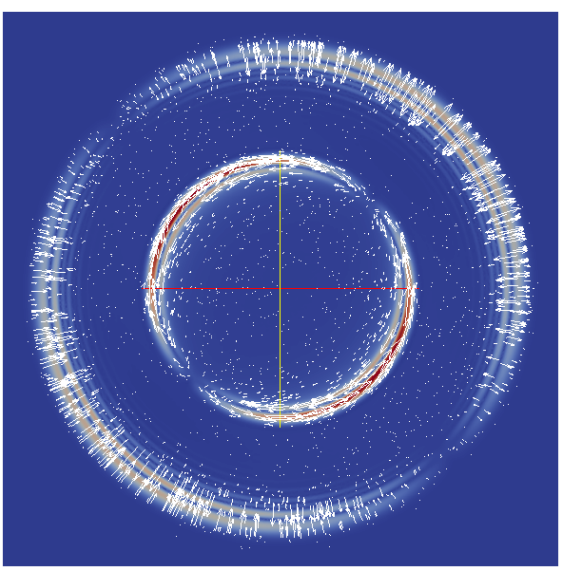

(d) Deterministic: Glyph

$\mathrm{km}$

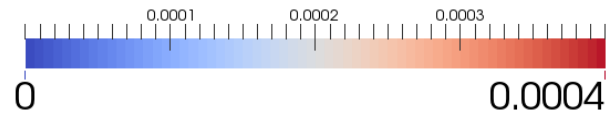

Figure 30: Magnitude of waves propagating in a homogeneous elastic soil medium: mean $\left(a_{0}\right)$ and deterministic output at $t=1.9 \mathrm{~s}$ 


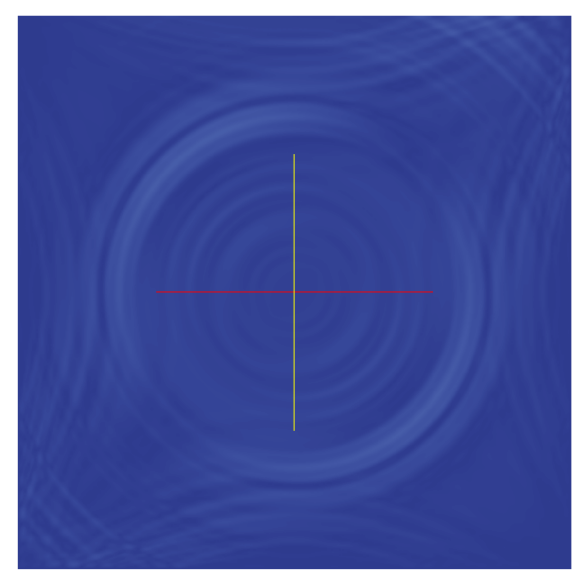

(a) Mean: Magnitude

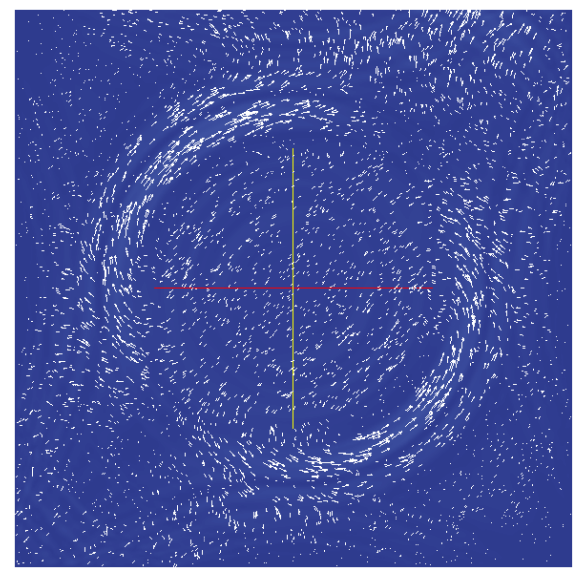

(b) Mean: Glyph

$\mathrm{km}$

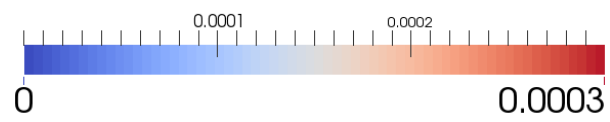

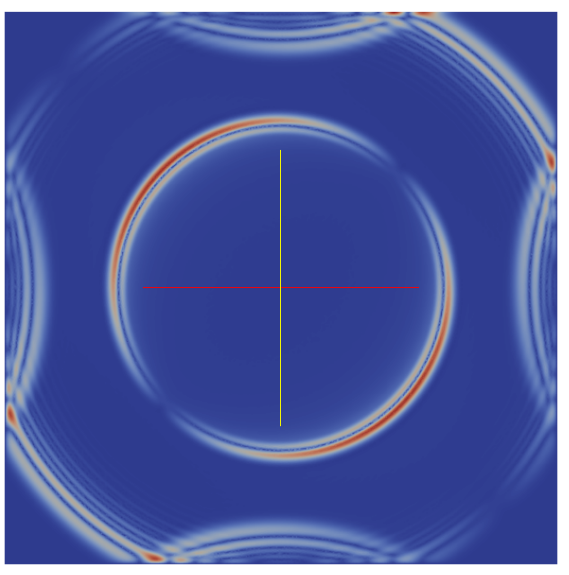

(c) Deterministic: Magnitude

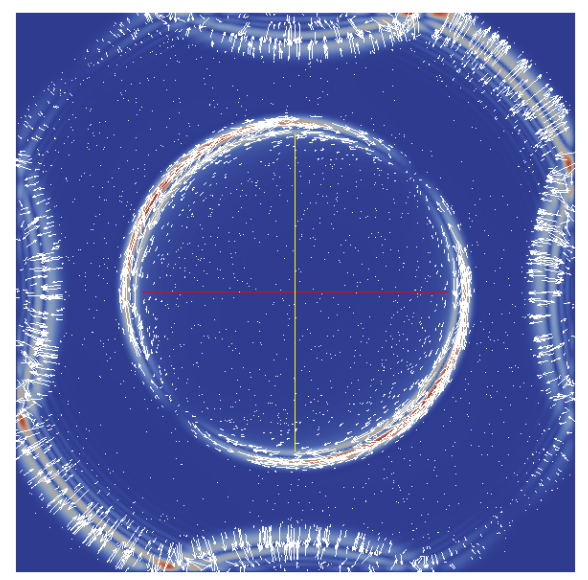

(d) Deterministic: Glyph

$\mathrm{km}$

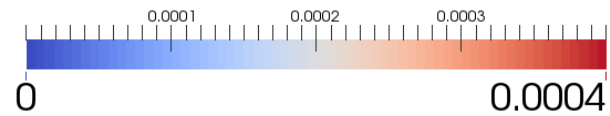

Figure 31: Magnitude of waves propagating in a homogeneous elastic soil medium: mean $\left(a_{0}\right)$ and deterministic output at $t=2.5 \mathrm{~s}$ 


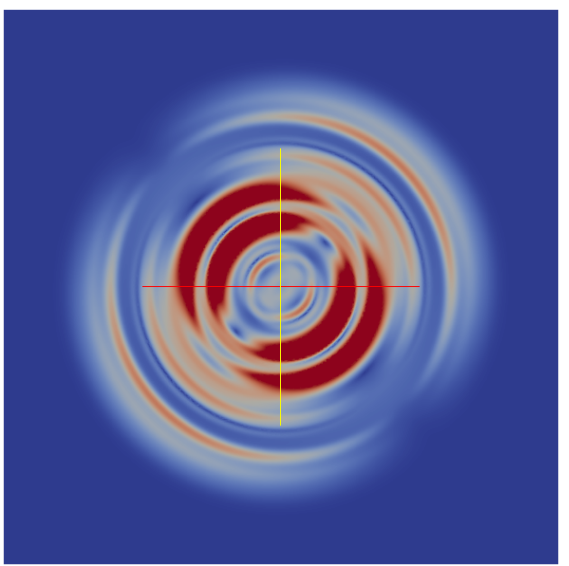

(a) $t=1.2 \mathrm{~s}$

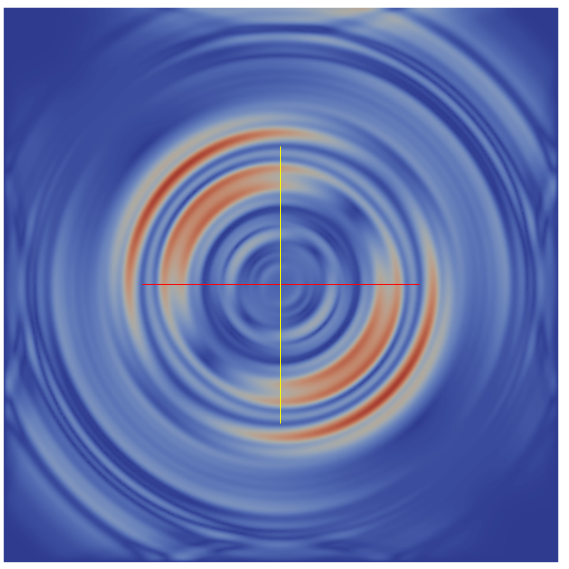

(c) $t=1.9 \mathrm{~s}$

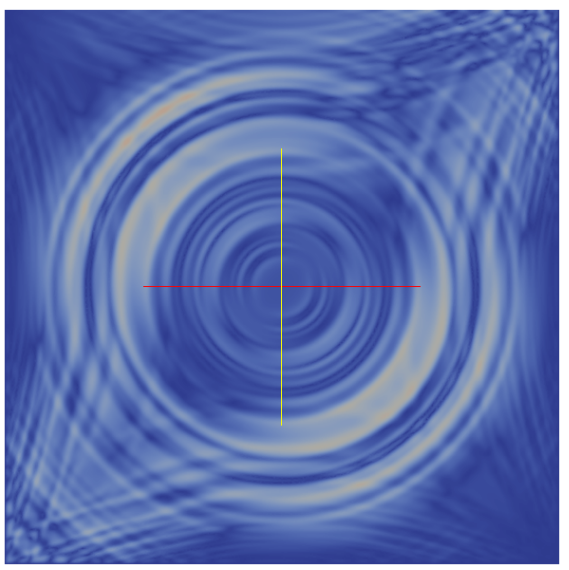

(e) $t=2.5 \mathrm{~s}$

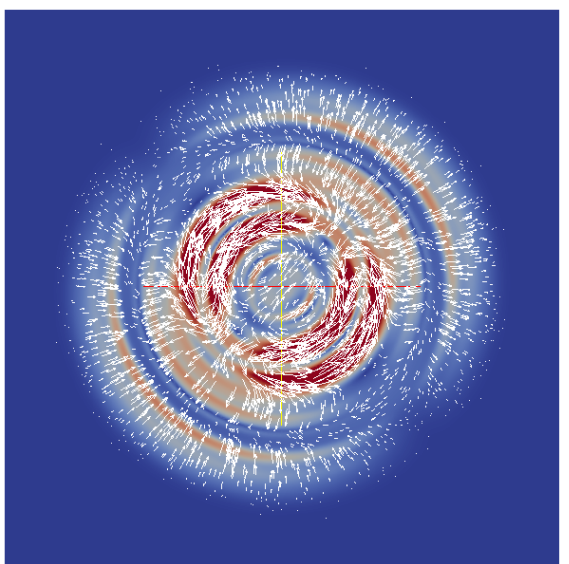

(b) $t=1.2 \mathrm{~s}$

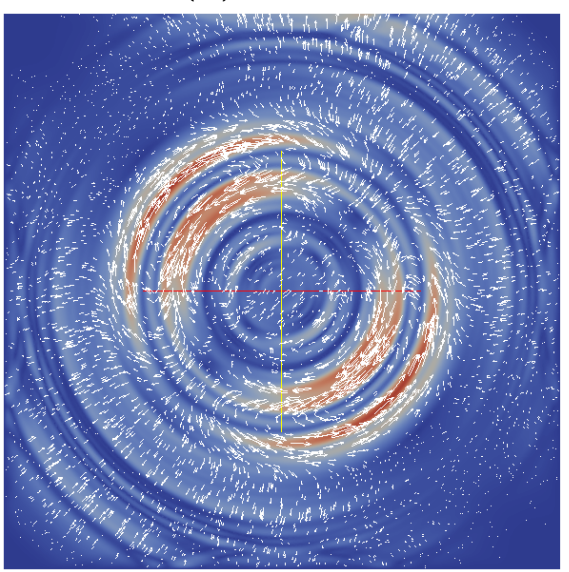

(d) $t=1.9 \mathrm{~s}$

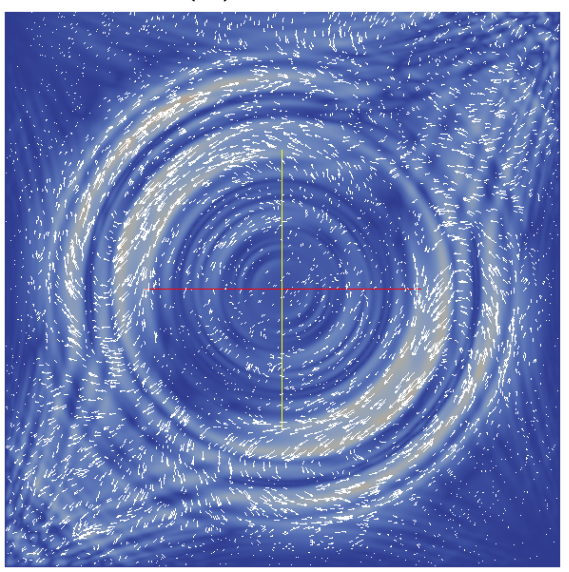

(f) $t=2.5 \mathrm{~s}$

$\mathrm{km}$

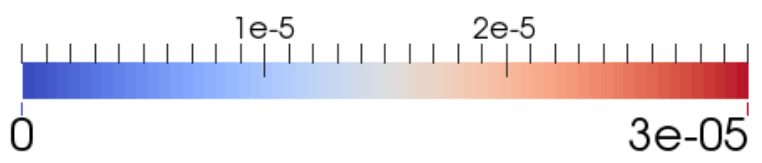

Figure 32: 1 st order PCE coefficient $a_{1}$ : Magnitude plots (left) and Glyph plots (right) 


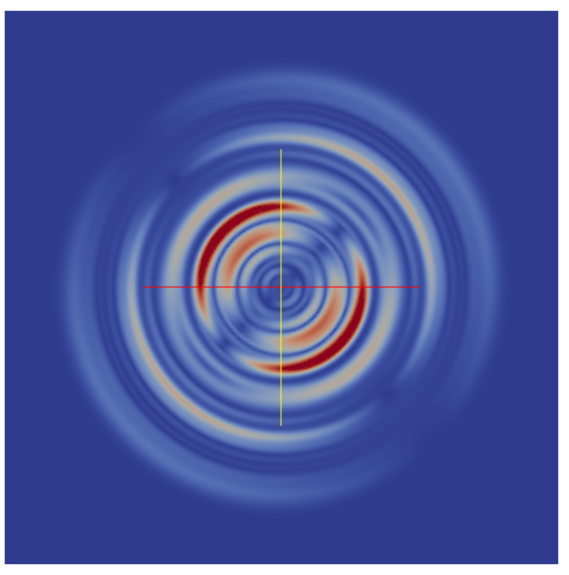

(a) $t=1.2 \mathrm{~s}$

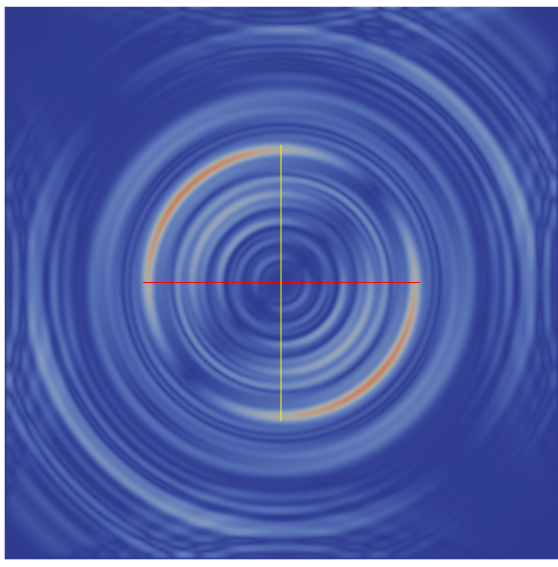

(c) $t=1.9 \mathrm{~s}$

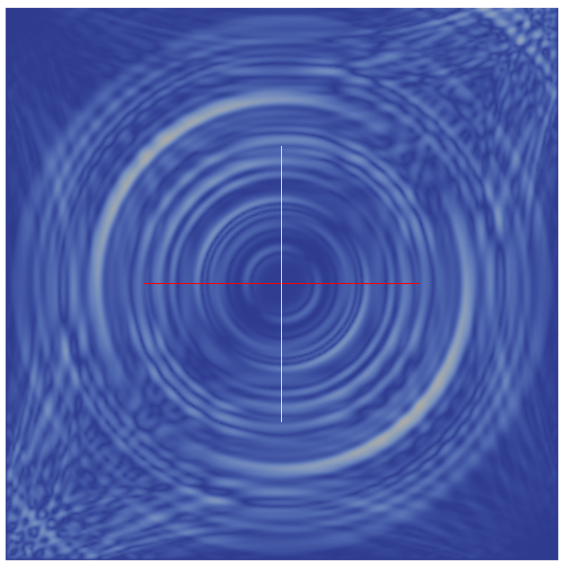

(e) $t=2.5 \mathrm{~s}$

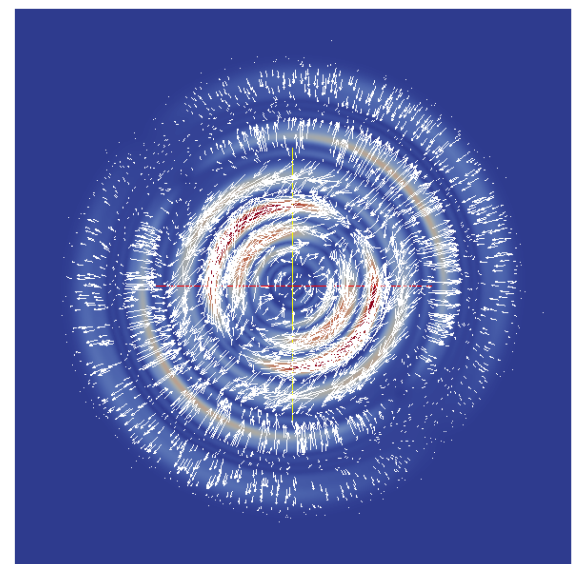

(b) $t=1.2 \mathrm{~s}$

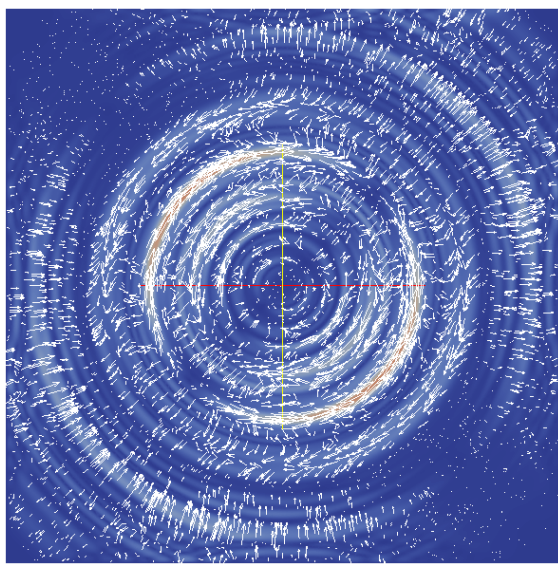

(d) $t=1.9 \mathrm{~s}$

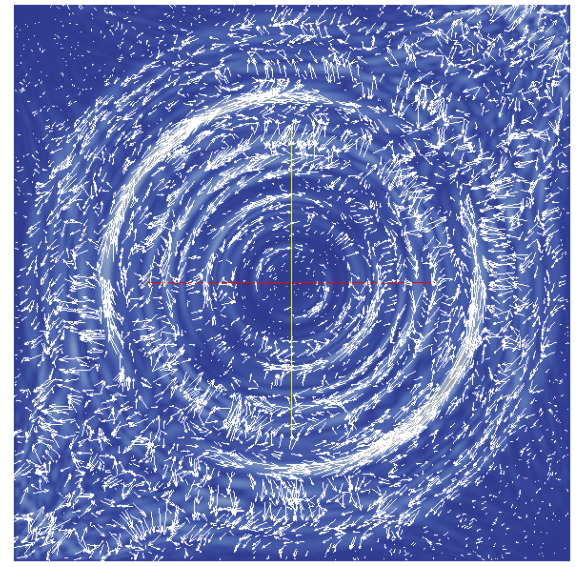

(f) $t=2.5 \mathrm{~s}$

$\mathrm{km}$

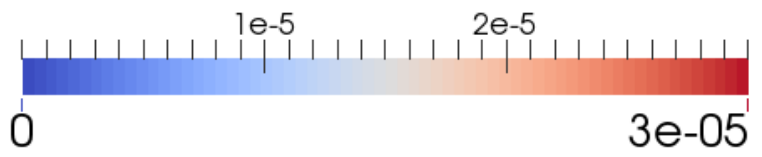

Figure 33: 2nd order PCE coefficient $a_{4}$ : Magnitude plots (left) and Glyph plots (right) 


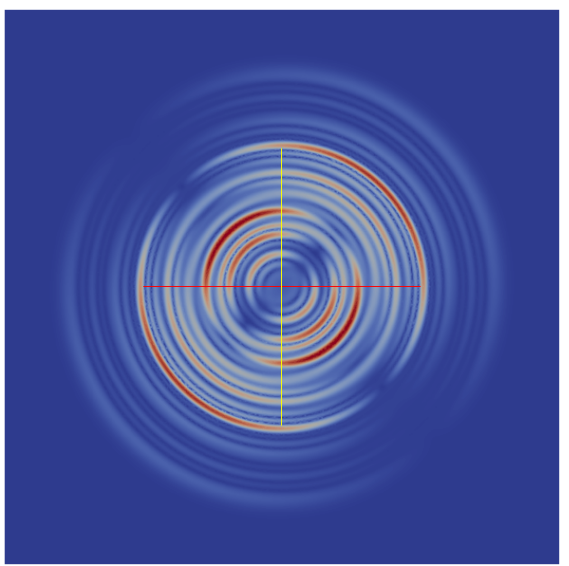

(a) $t=1.2 \mathrm{~s}$

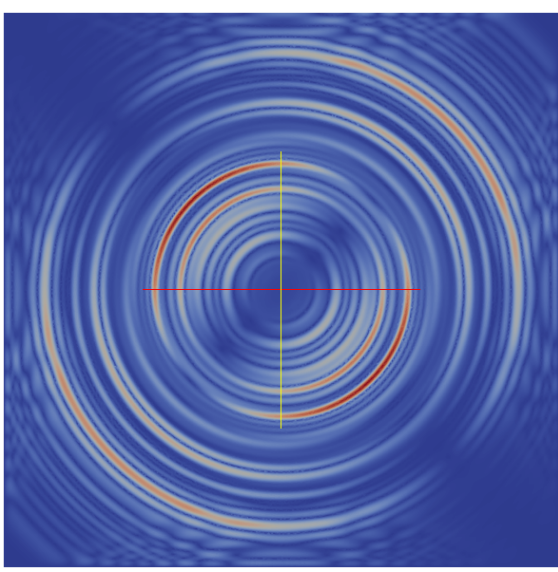

(c) $t=1.9 \mathrm{~s}$

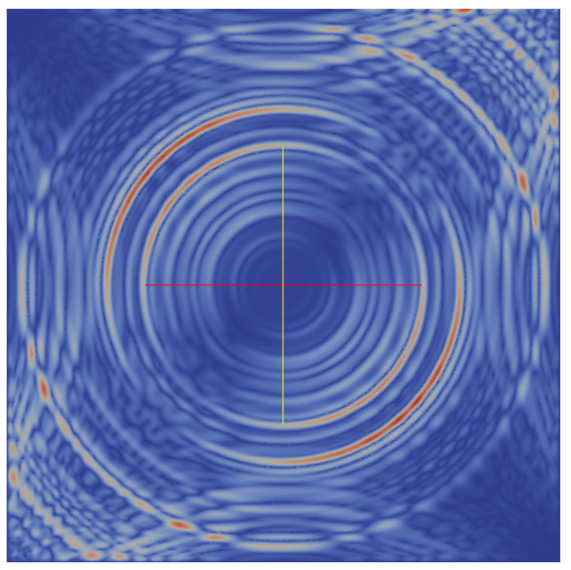

(e) $t=2.5 \mathrm{~s}$

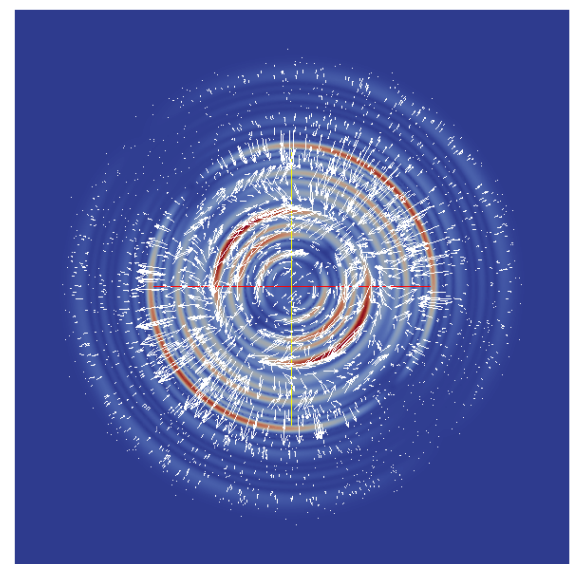

(b) $t=1.2 \mathrm{~s}$

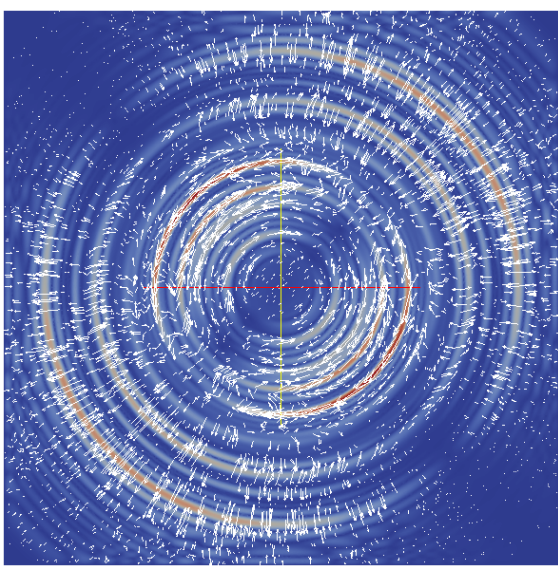

(d) $t=1.9 \mathrm{~s}$

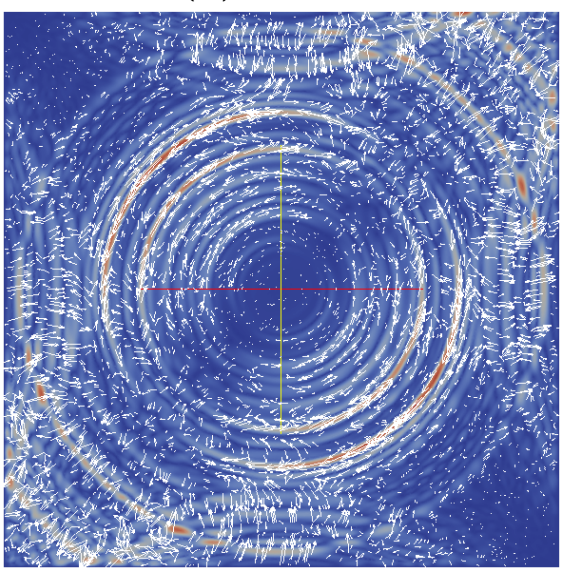

(f) $t=2.5 \mathrm{~s}$

$\mathrm{km}$

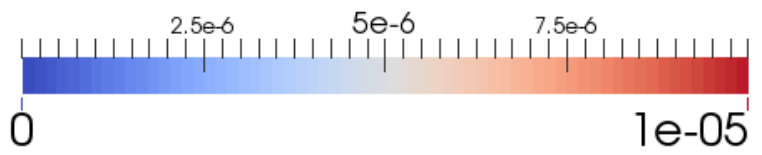

Figure 34: 2nd order PCE coefficient $a_{5}$ : Magnitude plots (left) and Glyph plots (right) 


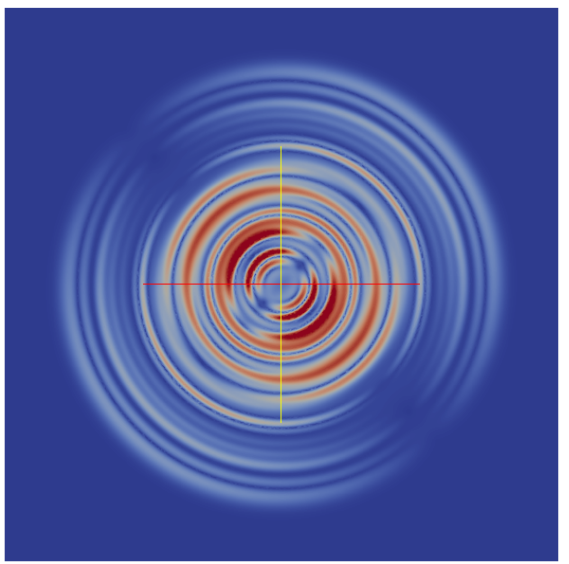

(a) $t=1.2 \mathrm{~s}$

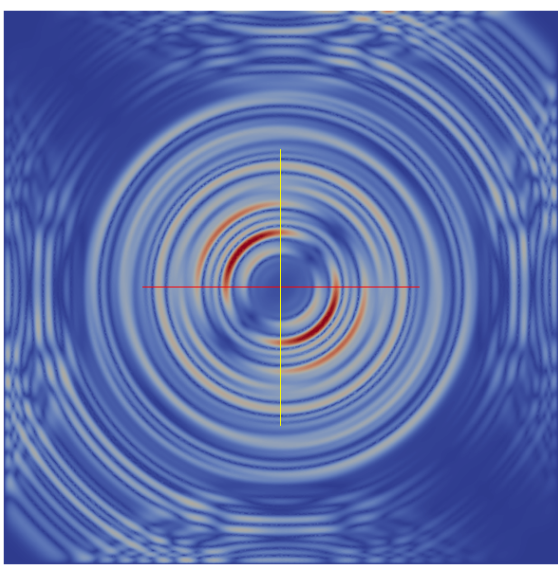

(c) $t=1.9 \mathrm{~s}$

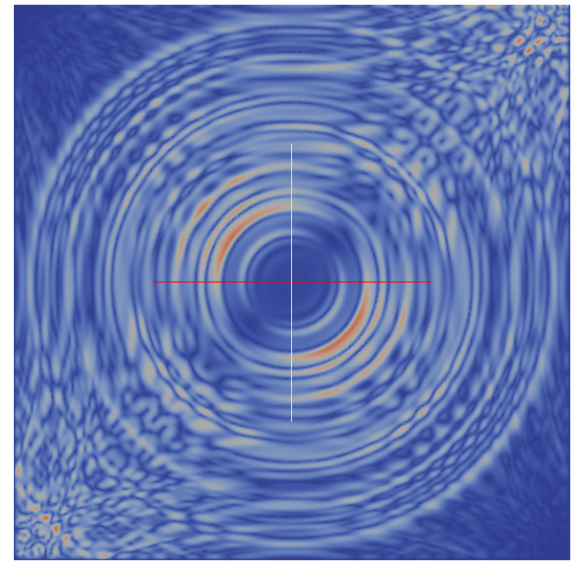

(e) $t=2.5 \mathrm{~s}$

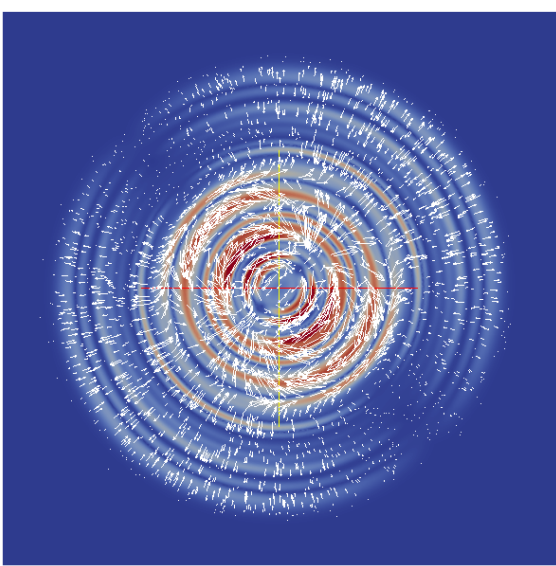

(b) $t=1.2 \mathrm{~s}$

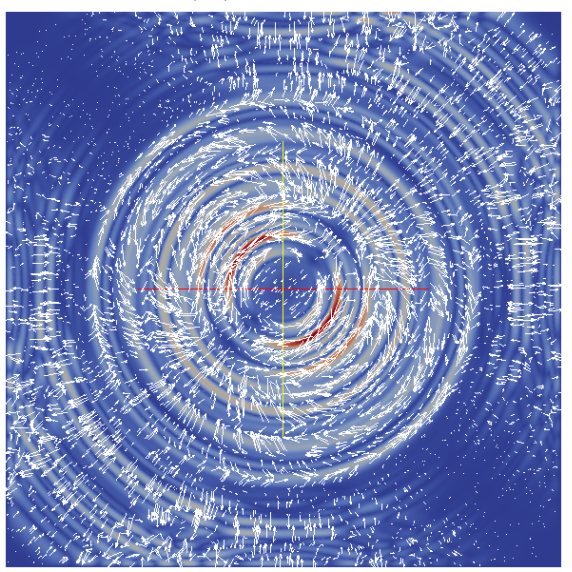

(d) $t=1.9 \mathrm{~s}$

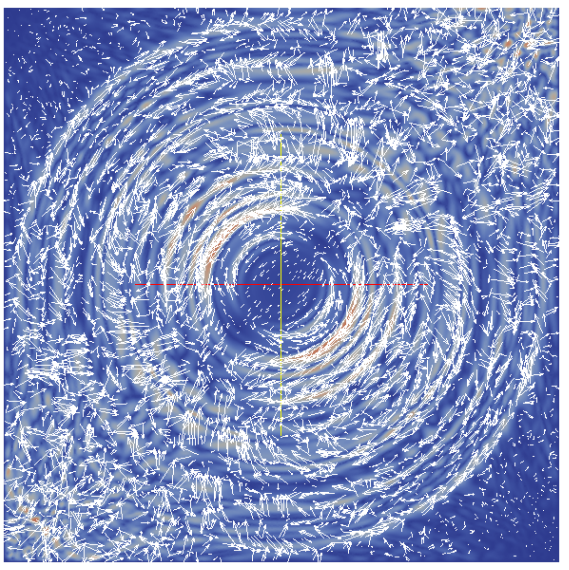

(f) $t=2.5 \mathrm{~s}$

$\mathrm{km}$

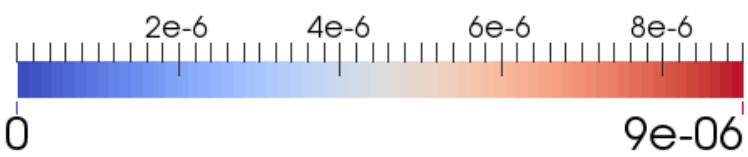

Figure 35: 3rd order PCE coefficient $a_{8}$ : Magnitude plots (left) and Glyph plots (right) 


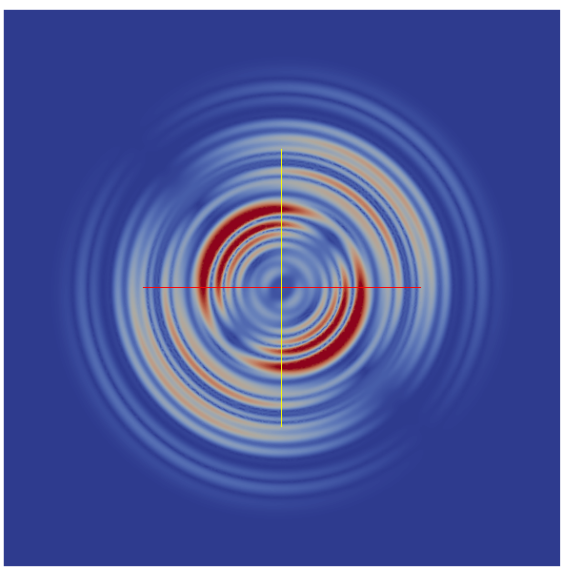

(a) $t=1.2 \mathrm{~s}$

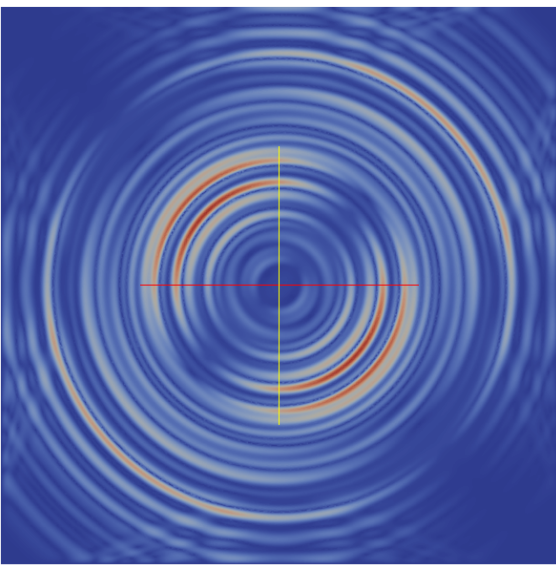

(c) $t=1.9 \mathrm{~s}$

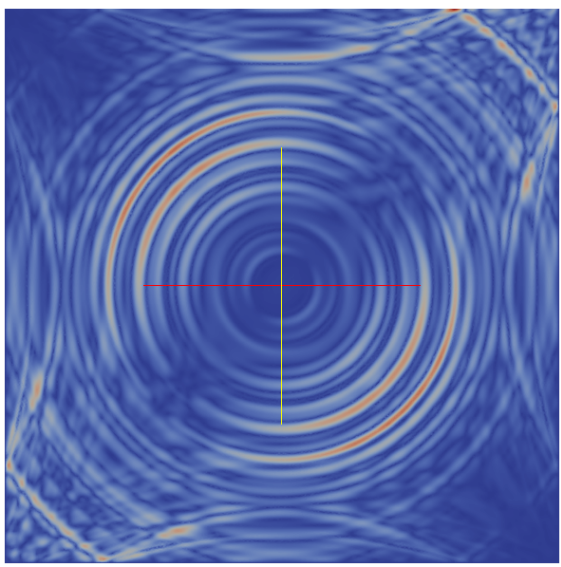

(e) $t=2.5 s$

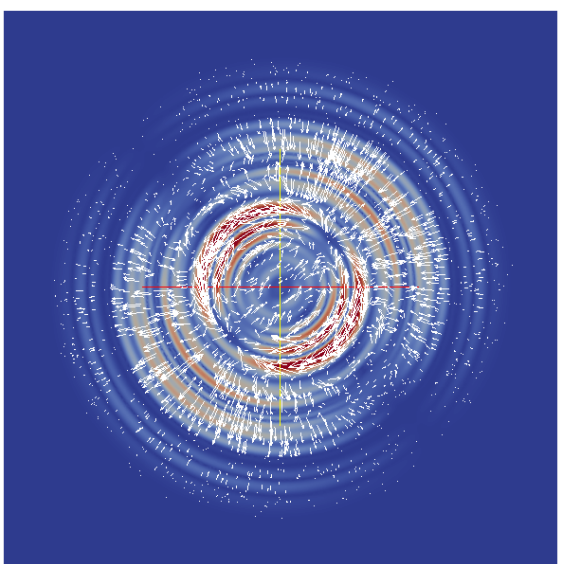

(b) $t=1.2 \mathrm{~s}$

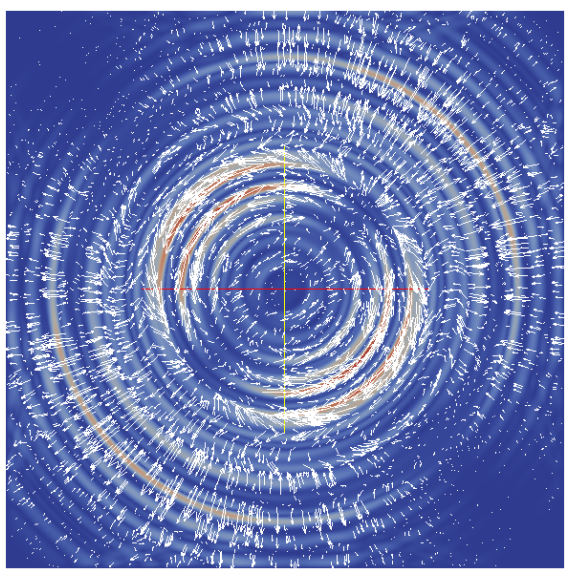

(d) $t=1.9 \mathrm{~s}$

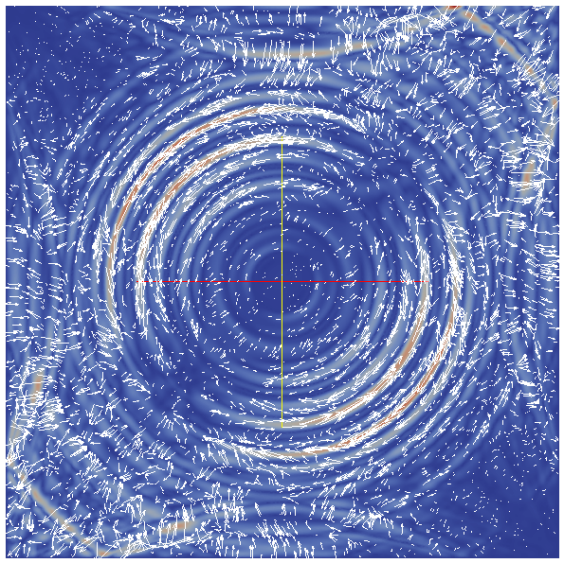

(f) $t=2.5 \mathrm{~s}$

$\mathrm{km}$

$2 e-6 \quad 4 e-6 \quad 6 e-6 \quad 8 e-6$

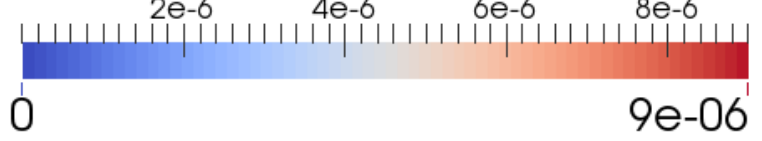

Figure 36: 3rd order PCE coefficient $a_{9}$ : Magnitude plots (left) and Glyph plots (right) 


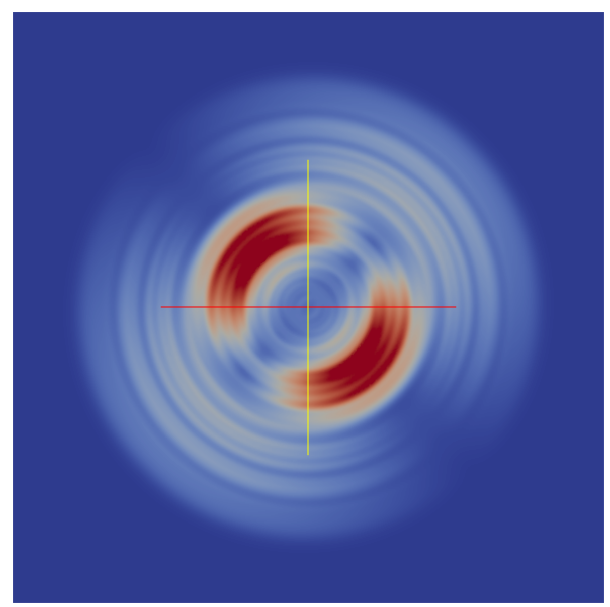

(a) $t=1.2 \mathrm{~s}$

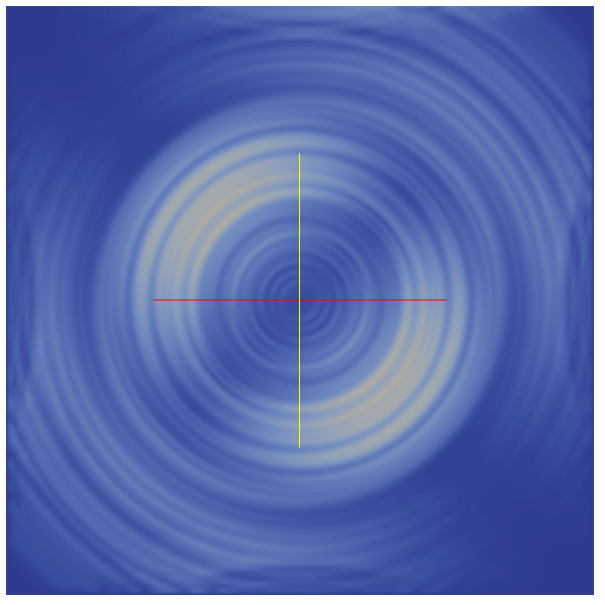

(b) $t=1.9 \mathrm{~s}$

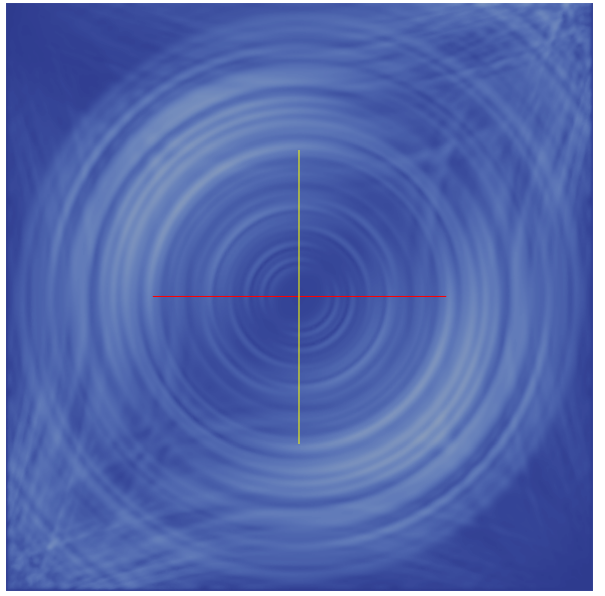

(c) $t=2.5 \mathrm{~s}$

$$
\mathrm{km}
$$

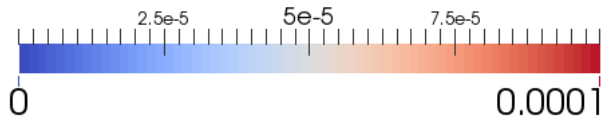

Figure 37: Standard deviation for the linear elastic model (2 random variables and 3rd order expansion) 


\subsubsection{Case 2: Linear wave propagation in layered piecewise homogeneous media}

We consider the case of waves propagating in a linear elastic layered piecewise homogeneous soil medium. The $36 \mathrm{~m}$ deep soil profile is based on the Victoria Memorial Museum site in Ottawa (as described in [174]). The top soil layer is $2.5 m$ thick and is followed by a $20.5 m$ thick layer of clayey silt. Below this layer lies a $13 m$ transition zone composed of clayey silt, sandy silt and glacial till at the bottom. For each layer, two physical input parameters, i.e., the Young's modulus $E$ and the density $\rho$ have been considered as random variables. Therefore, a total of six random variables have been considered for three soil layers while the bedrock parameters remain constant. Truncated Gaussian distributions are assumed for $E$ and $\rho$ such that all values remain positive. The assumed mean values and COVs for the input parameters used in the simulation are given in the Table 5. A mesh of size $[200,150] \mathrm{m}^{2}$ discretized into approximately 220,000 triangular cells was used for this simulation and the source pulse was placed in the rock layer just below the glacial till (at a depth of approximately $40 m$ ). A proportionate mesh depicting different layers and the location of the source has been shown in Fig. 38. It can be observed that the mesh has been refined at interfaces of different layers so that wave reflections and refractions can be adequately captured.

A comparison of the deterministic and the mean solution from PCE has been shown in Fig. 39-42. Though the estimated mean response looks nearly identical to the deterministic response in the bedrock layer, it is clearly lower in magnitude for the waves propagating in the soil layers above the rock. It can be observed that as the waves transit from rock into the lowest soil layer, the wave profile and velocity changes (becomes slower). Reflections and refractions can be noticed at the interface of the 


\begin{tabular}{|c|c|c|c|}
\hline Layer Depth $(m)$ & Parameter & Mean $\mu$ & COV $(\%)$ \\
\hline \multirow{2}{*}{2.5} & $E_{1}$ & $65 \mathrm{MPa}$ & 40 \\
& $\rho_{1}$ & $1600 \mathrm{~kg} / \mathrm{m}^{3}$ & 10 \\
\hline \multirow{2}{*}{20.5} & $E_{2}$ & $160 \mathrm{MPa}$ & 40 \\
& $\rho_{2}$ & $1700 \mathrm{~kg} / \mathrm{m}^{3}$ & 10 \\
\hline \multirow{2}{*}{13} & $E_{3}$ & $281 \mathrm{MPa}$ & 40 \\
& $\rho_{3}$ & $1800 \mathrm{~kg} / \mathrm{m}^{3}$ & 10 \\
\hline \multirow{2}{*}{ Bedrock } & $E_{4}$ & $14625 \mathrm{MPa}$ & - \\
& $\rho_{4}$ & $2500 \mathrm{~kg} / \mathrm{m}^{3}$ & - \\
\hline
\end{tabular}

Table 5: Values of mean and COV for parameters of the layered linear elastic model

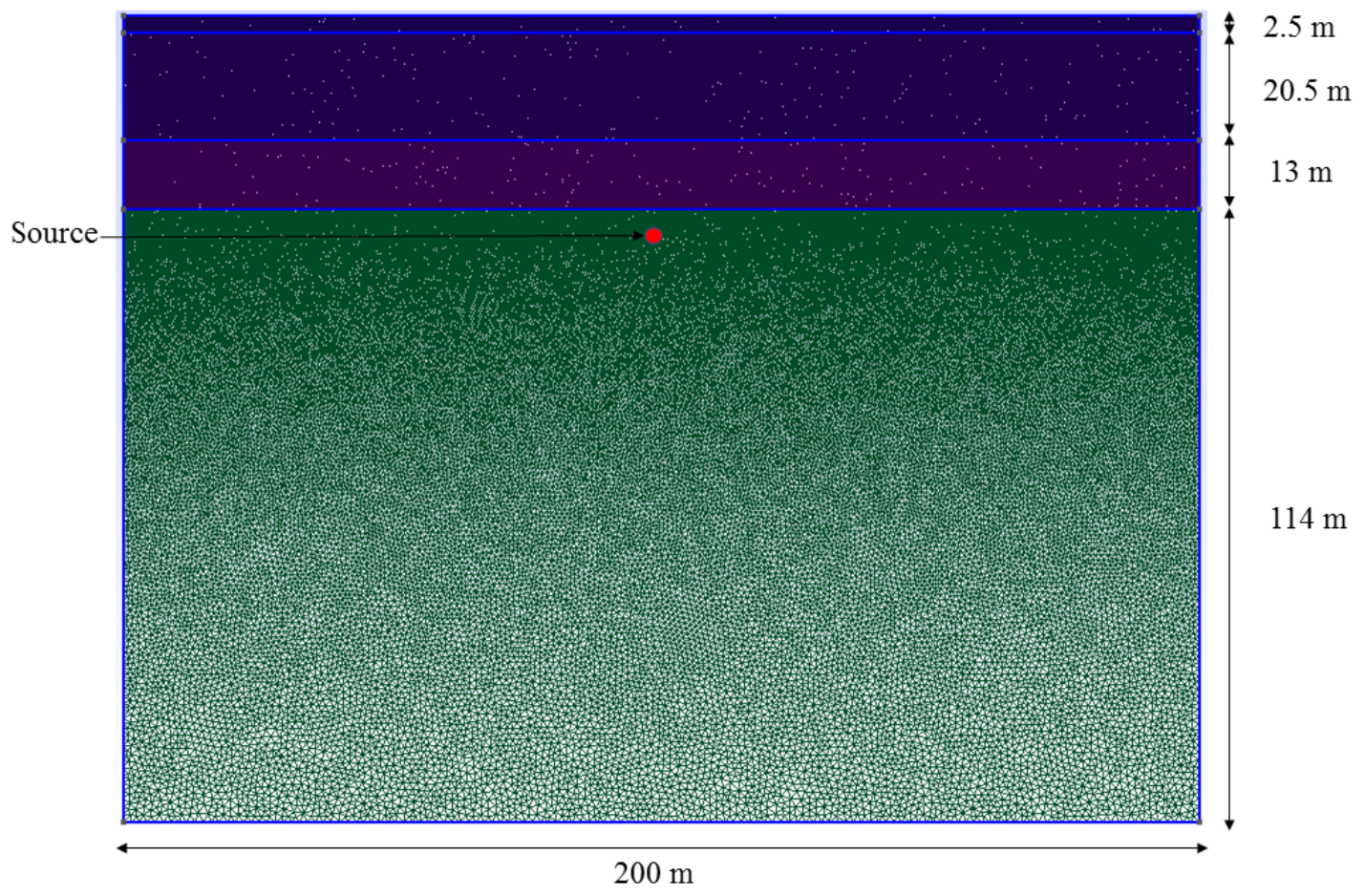

Figure 38: Mesh for layered soil profile 
two layers. As time evolves, the behaviour appears to be similar to soil amplification since the contrast in shear wave velocity of rock and soil causes entrapment of the seismic waves in the soil layers. PCE coefficients from 1st to 3rd order have been shown in Fig. 43-48. It can be observed how different coefficients contribute to form the solution.

\subsubsection{Case 3: Non-linear elasto-plastic wave propagation in homogeneous media}

Now we consider the case of waves propagating in an isotropic homogeneous elastoplastic soil medium having random parameters. The rate independent associative Drucker Prager constitutive model with isotropic hardening has been implemented. For this case, four physical input parameters, i.e., the Young's modulus E, density $\rho$, cohesion $c$, and angle of friction $\phi$ have been considered as random variables. A truncated Gaussian distribution is assumed such that all the values remain positive. The assumed mean values and COVs for the input parameters used in the simulation are shown in Table 6. A comparison of the deterministic and the mean solution from PCE has been shown in Fig. 49-51. Similar to the linear elastic case, here too the estimated mean particle displacement contours show a lower magnitude and spatially distributed response compared to the respective deterministic response.

The PCE coefficients shown in Fig. 52 is one of the 1 st order coefficients which represent the Gaussian contribution to the solution, while the 2 nd and $3 r d$ order coefficients shown in Fig. 53-56 represent the non-Gaussian contribution. As stated earlier, the PCE coefficients are interesting to observe because they form a certain contribution to the parametrised solution and can be used to cheaply reconstruct innumerable potential solutions based on newly generated random samples. It can be 
(a) Mean: Magnitude

(c) Deterministic: Magnitude

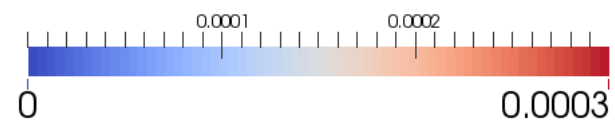$$
\mathrm{km}
$$
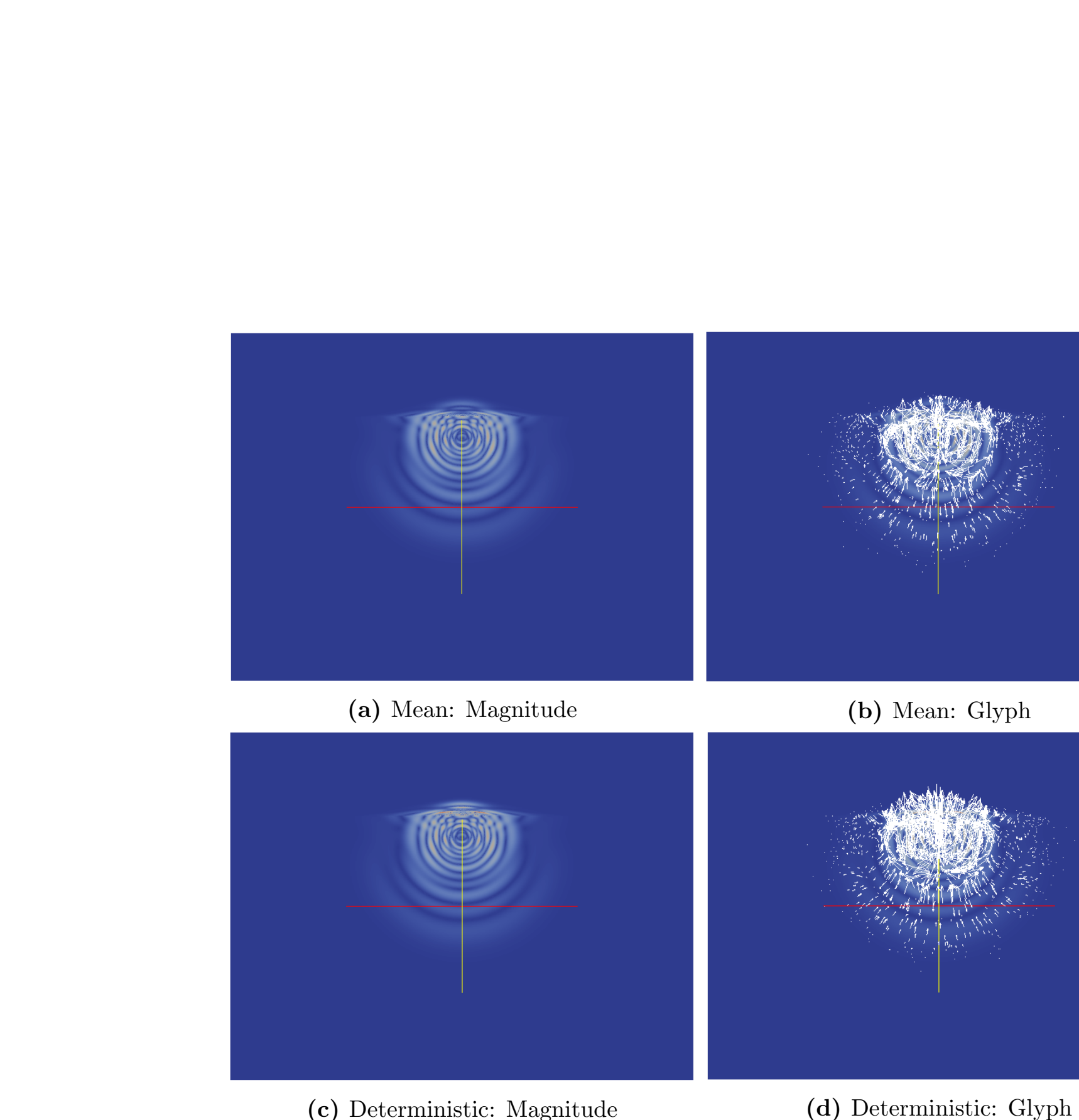

(b) Mean: Glyph

(d) Deterministic: Glyph
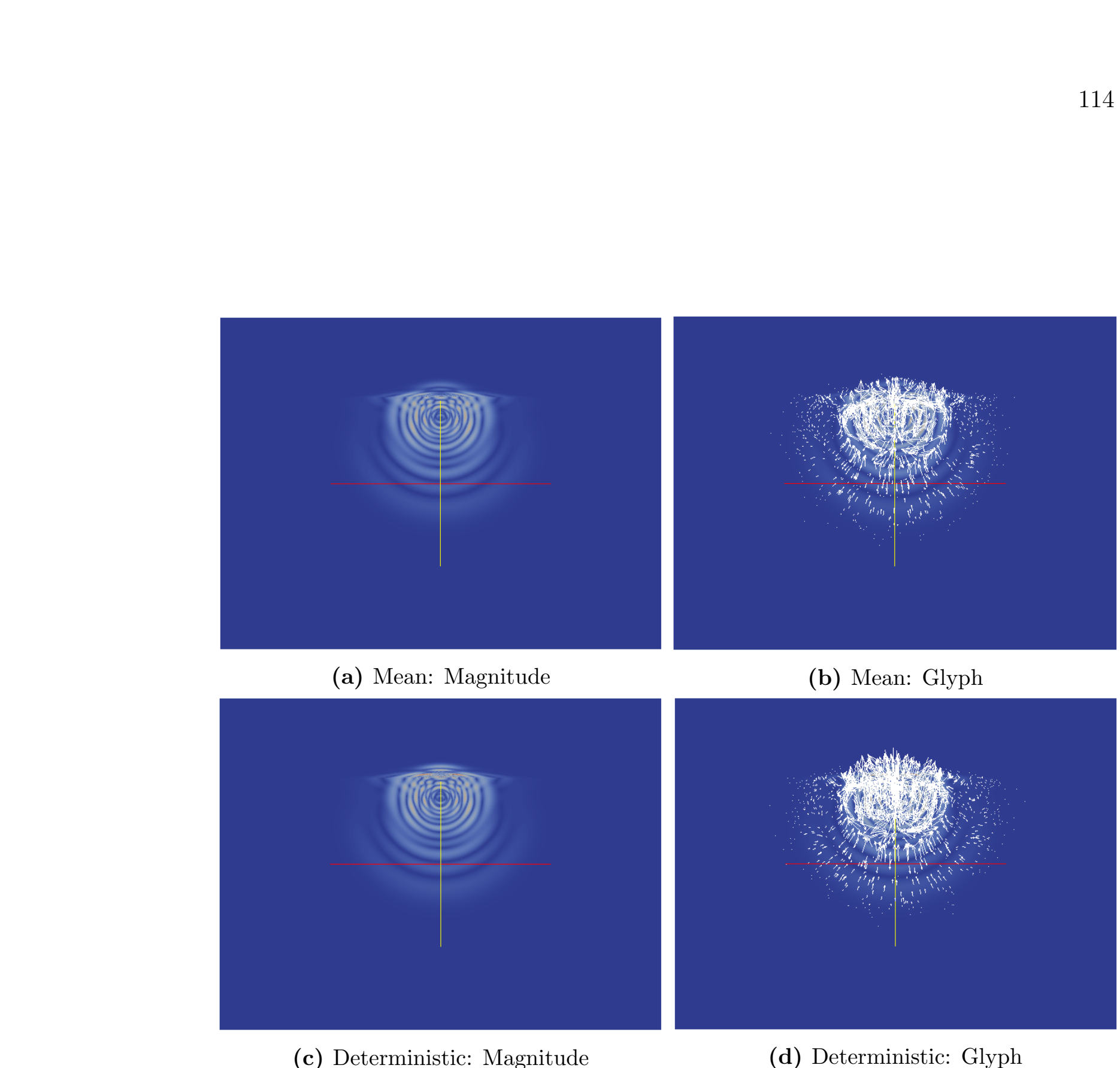

(14) 


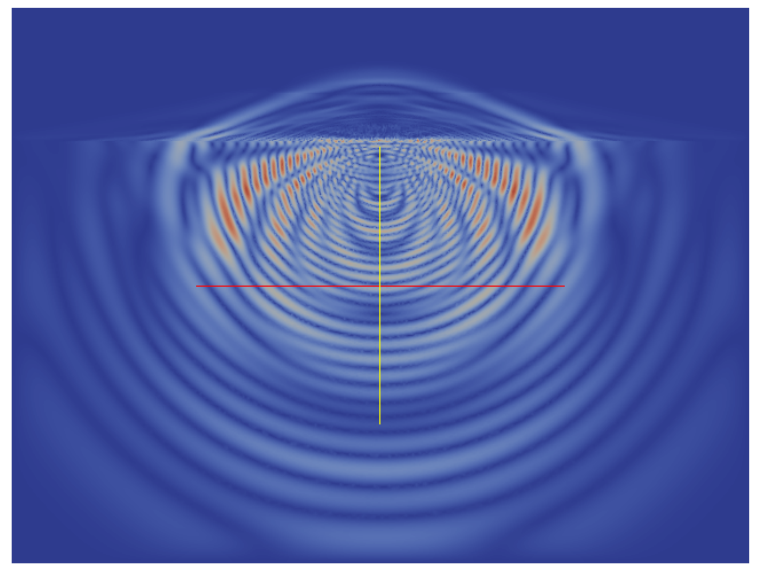

(a) Mean: Magnitude

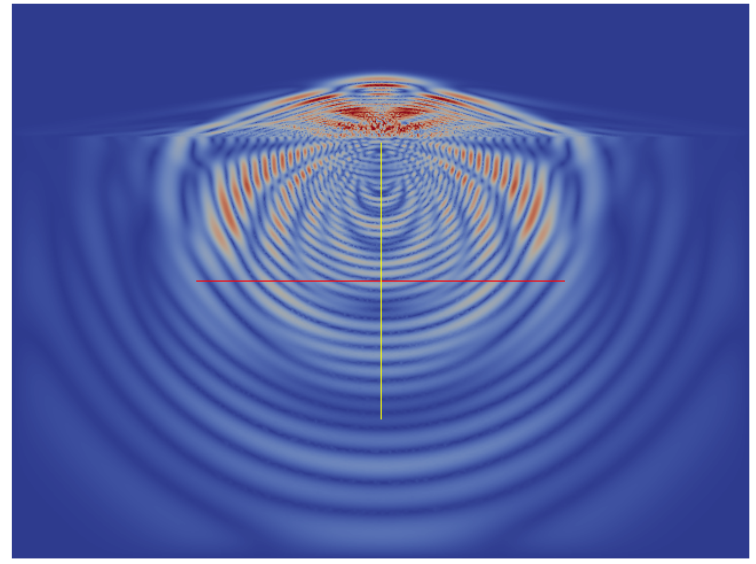

(c) Deterministic: Magnitude

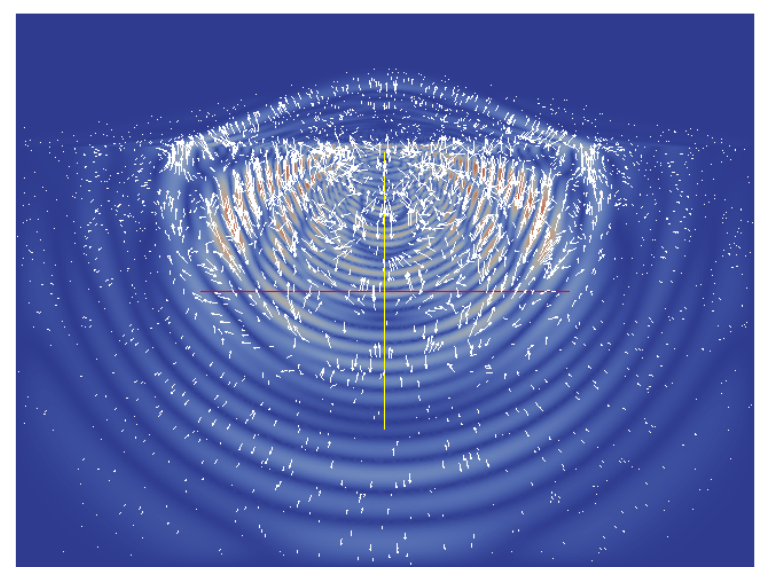

(b) Mean: Glyph

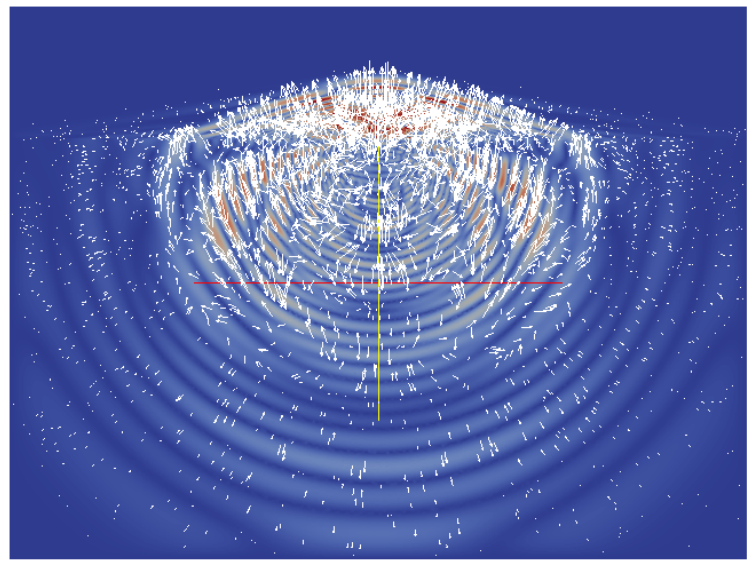

(d) Deterministic: Glyph

$\mathrm{km}$

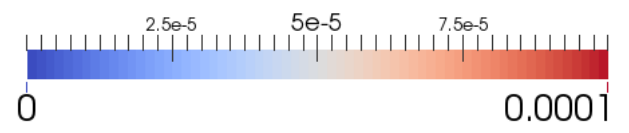

Figure 40: Magnitude of waves propagating in a layered homogeneous elastic soil medium: mean $\left(a_{0}\right)$ and deterministic output at $t=0.041 \mathrm{~s}$ 


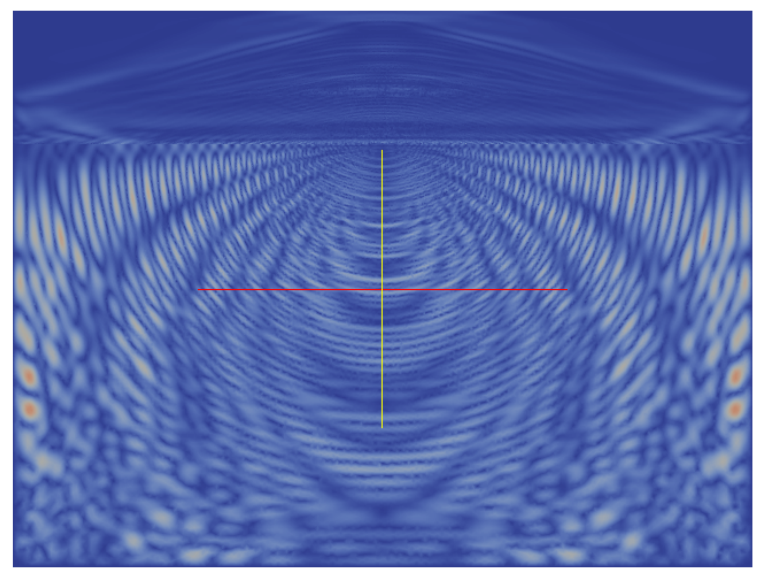

(a) Mean: Magnitude

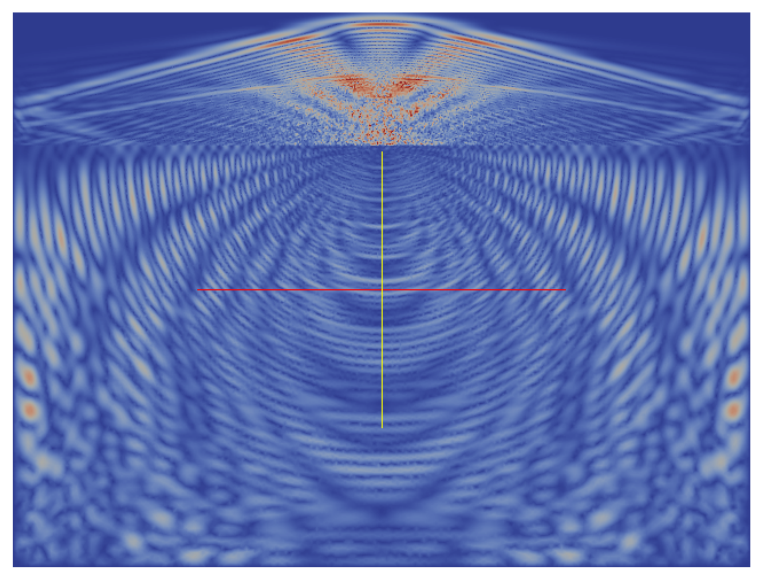

(c) Deterministic: Magnitude

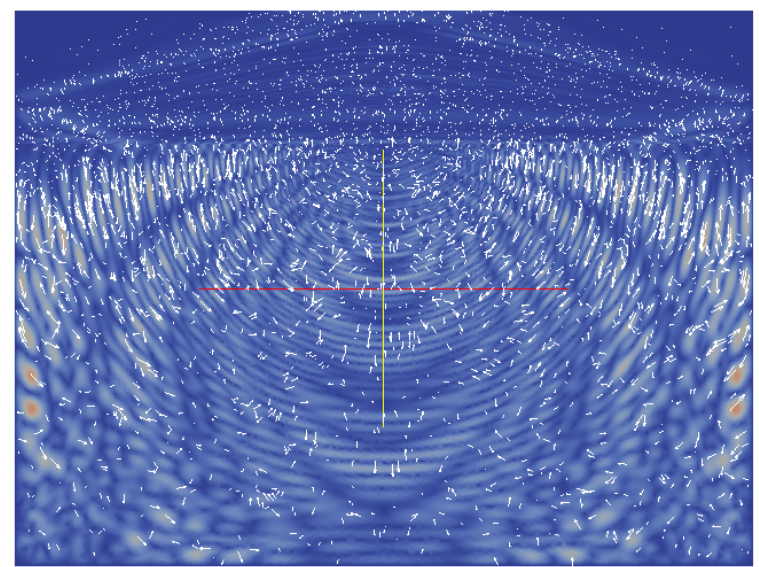

(b) Mean: Glyph

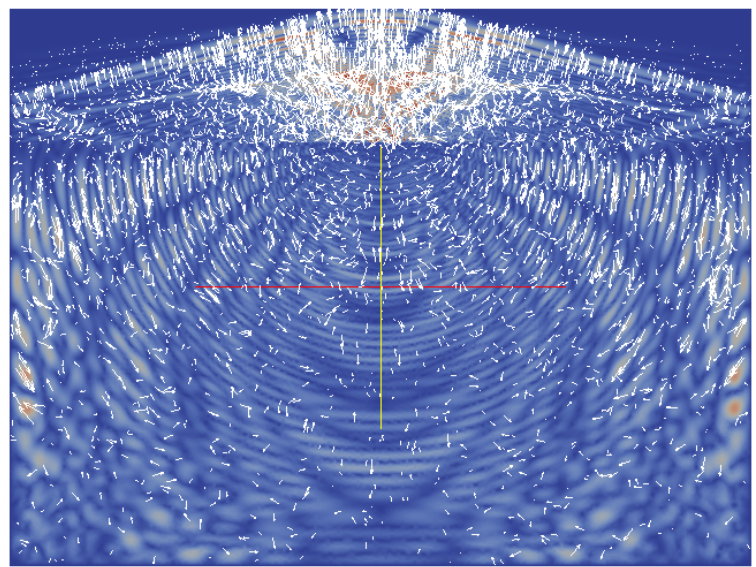

(d) Deterministic: Glyph

$\mathrm{km}$

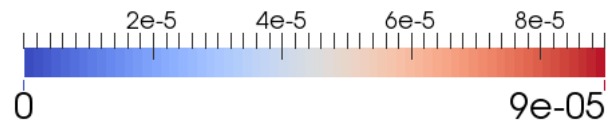

Figure 41: Magnitude of waves propagating in a layered homogeneous elastic soil medium: mean $\left(a_{0}\right)$ and deterministic output at $t=0.096 \mathrm{~s}$ 


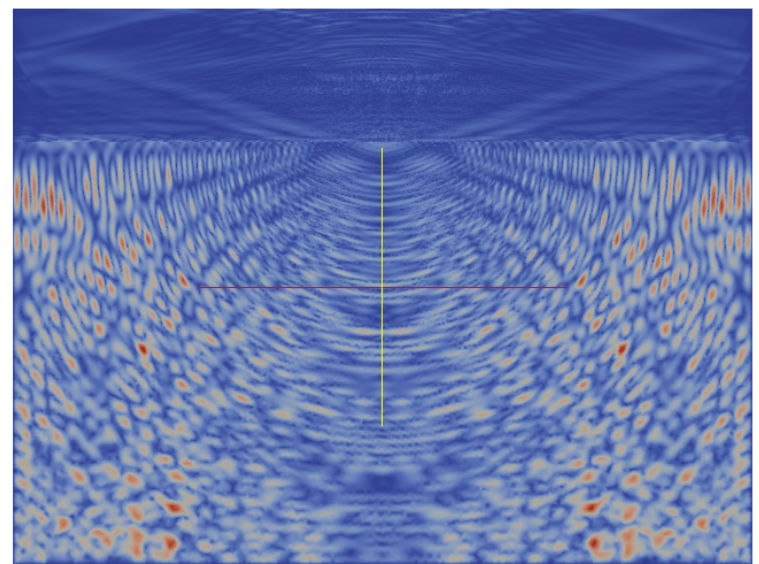

(a) Mean: Magnitude

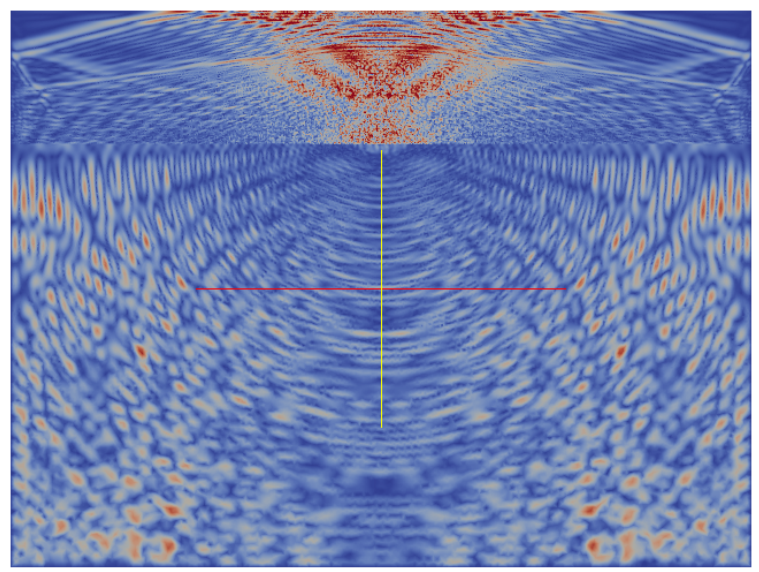

(c) Deterministic: Magnitude

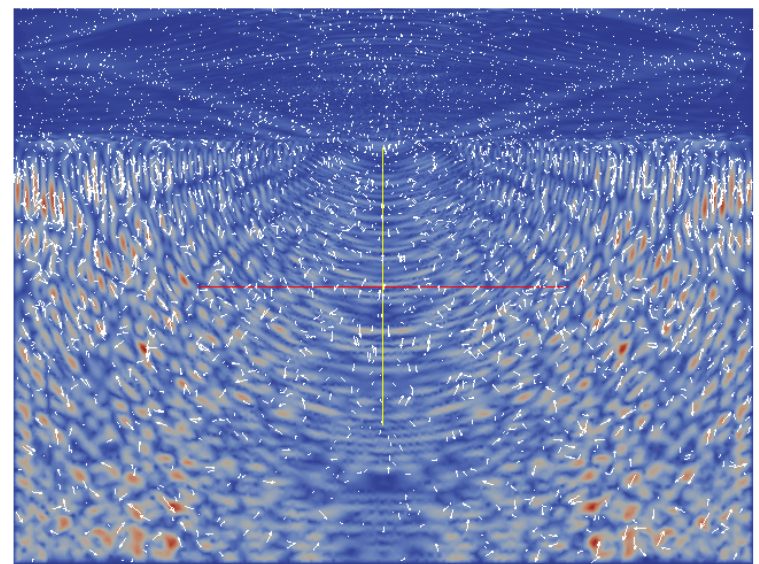

(b) Mean: Glyph

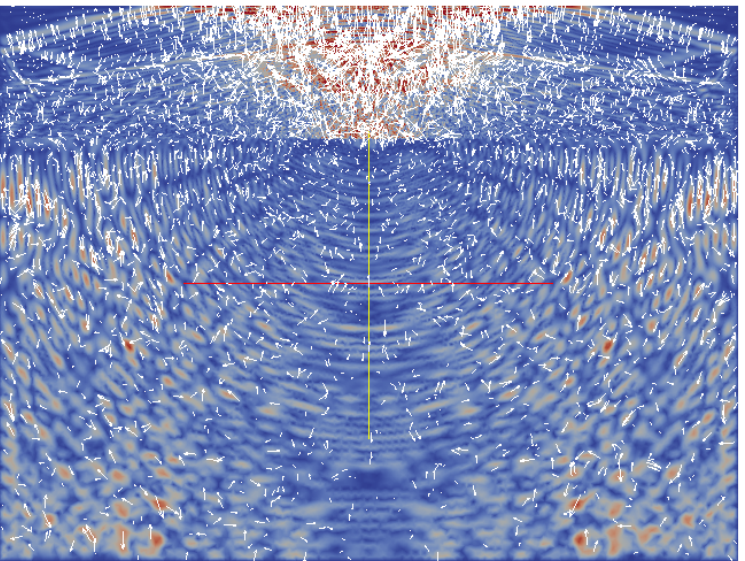

(d) Deterministic: Glyph

$\mathrm{km}$

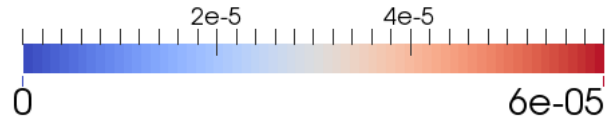

Figure 42: Magnitude of waves propagating in a layered homogeneous elastic soil medium: mean $\left(a_{0}\right)$ and deterministic output at $t=0.136 \mathrm{~s}$ 


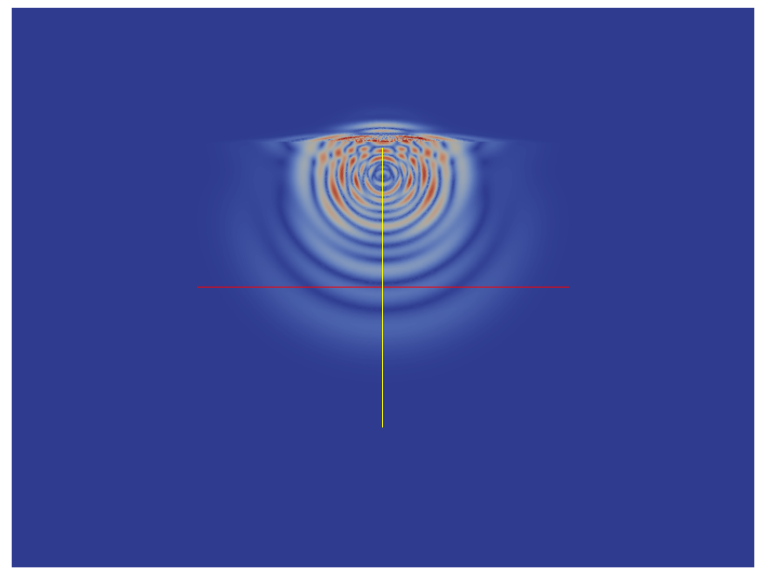

(a) $t=0.016 \mathrm{~s}$

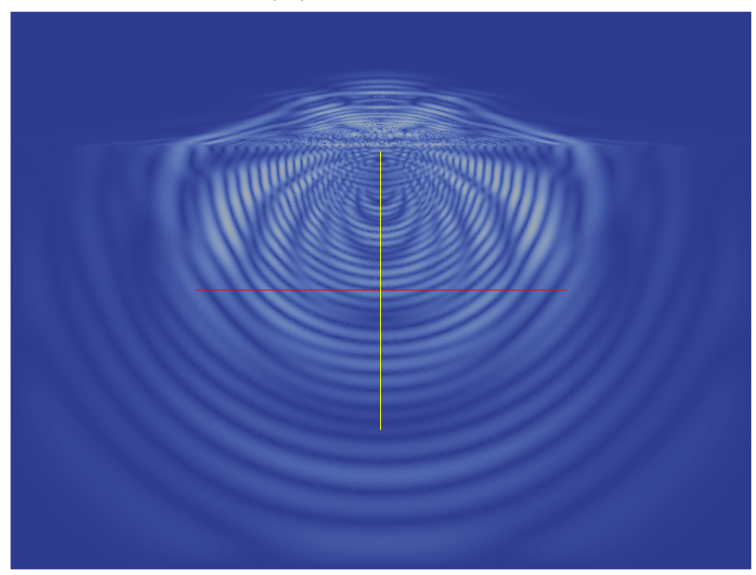

(c) $t=0.041 \mathrm{~s}$

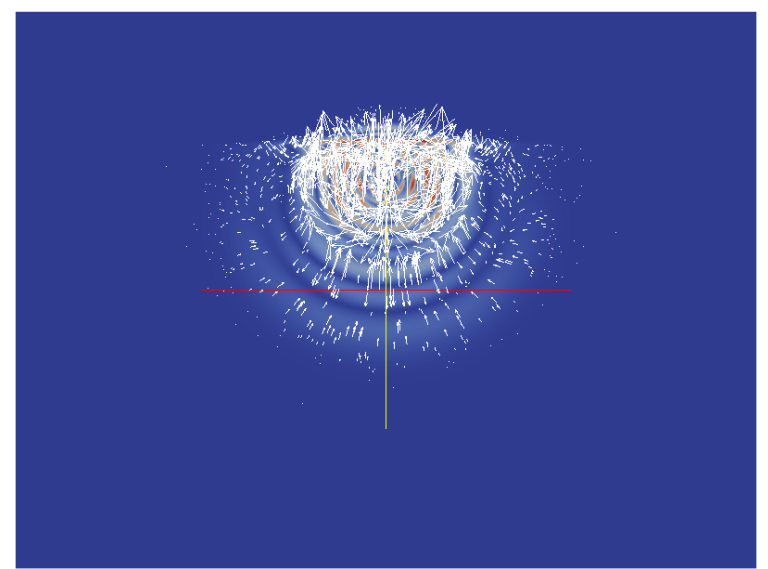

(b) $t=0.016 \mathrm{~s}$

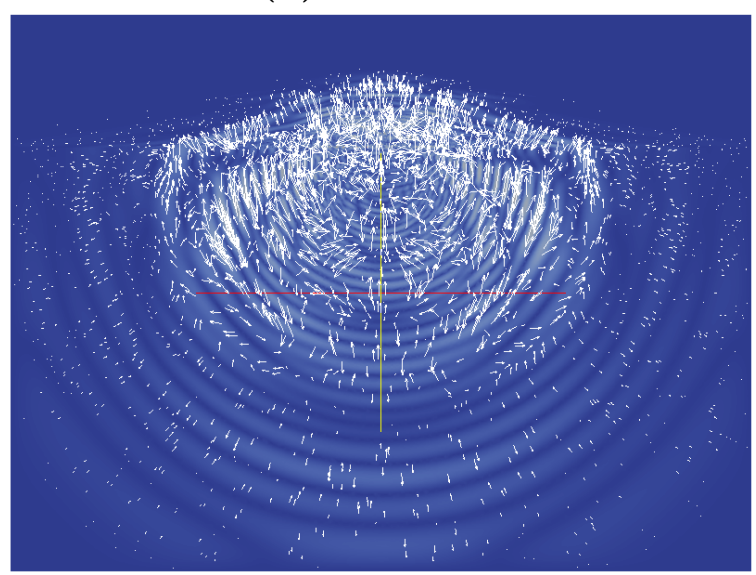

(d) $t=0.041 \mathrm{~s}$

$\mathrm{km}$

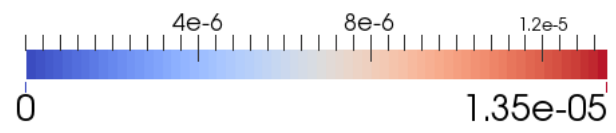

Figure 43: 1 st order PCE coefficient $a_{1}$ for case 2: Magnitude plots (left) and Glyph plots (right) 


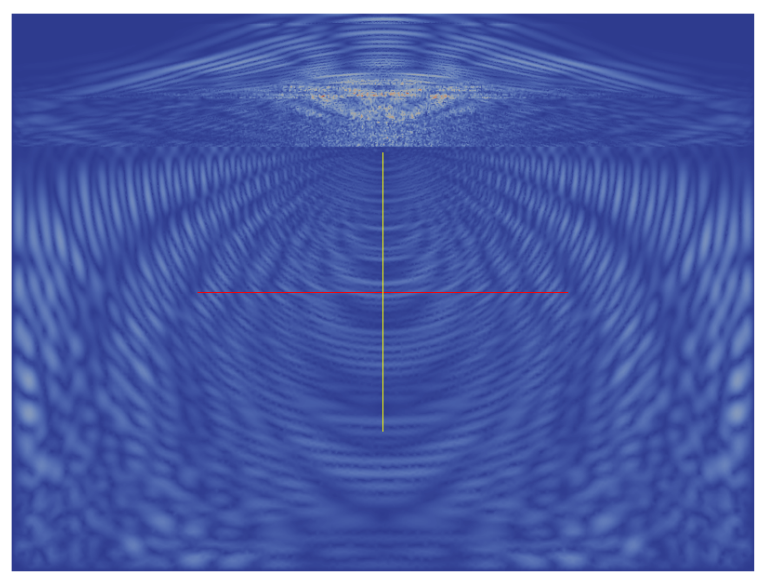

(a) $t=0.096 \mathrm{~s}$

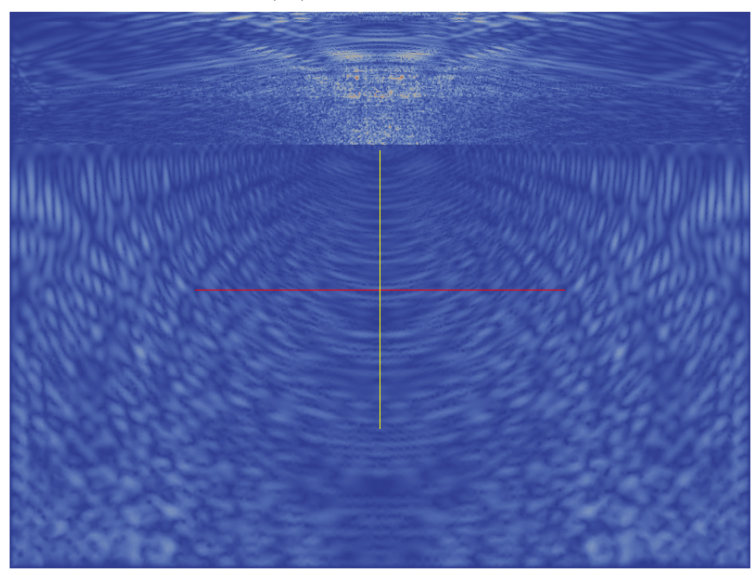

(c) $t=0.136 \mathrm{~s}$

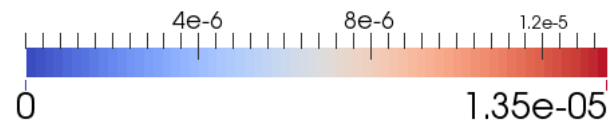

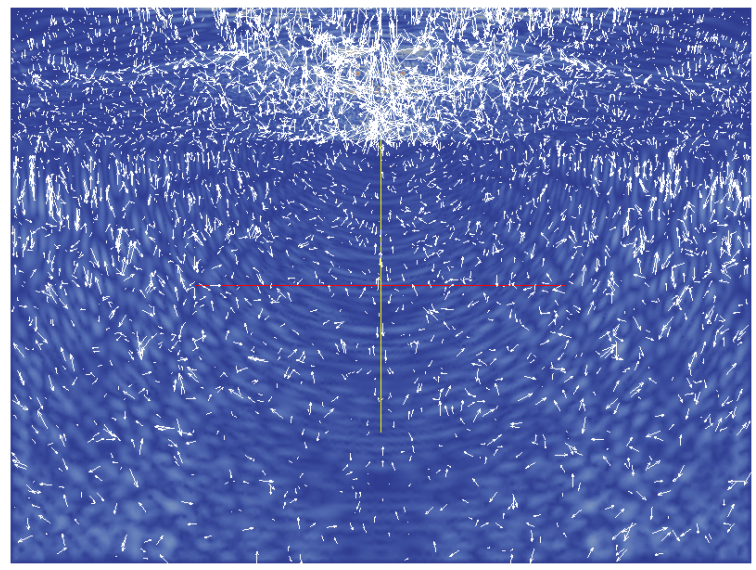

(d) $t=0.136 \mathrm{~s}$
(b) $t=0.096 \mathrm{~s}$

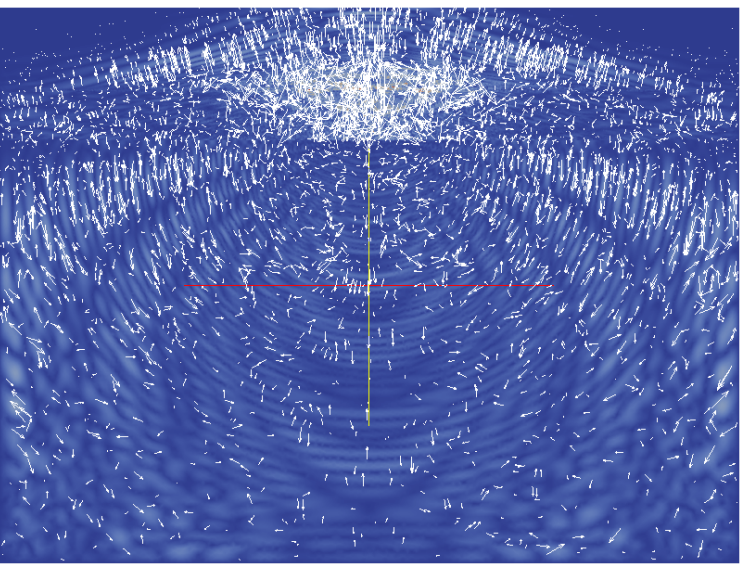




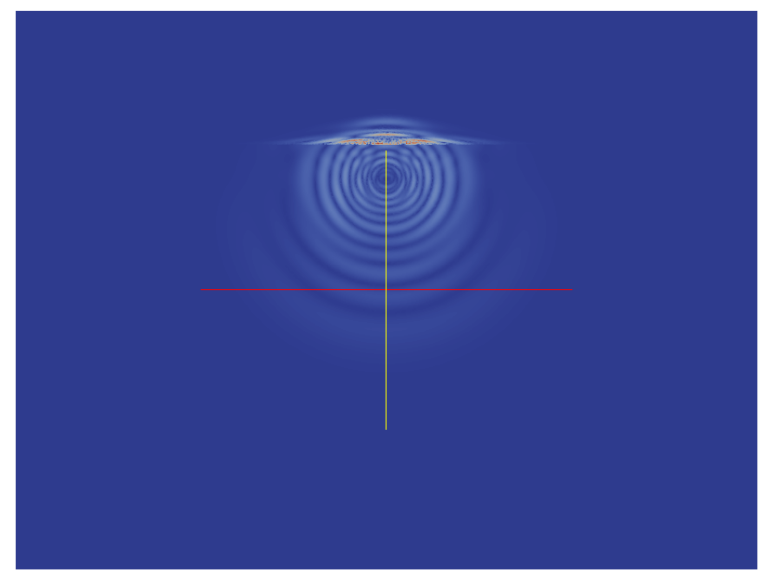

(a) $t=0.016 \mathrm{~s}$

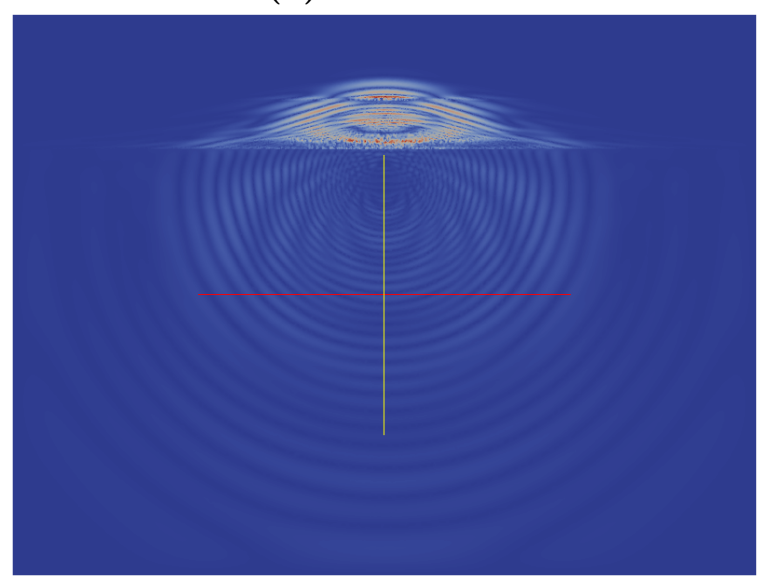

(c) $t=0.041 \mathrm{~s}$

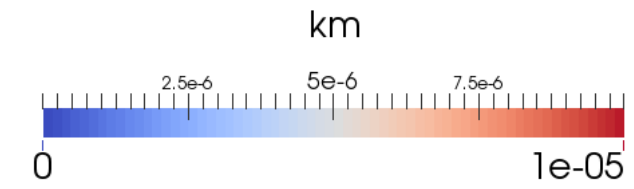

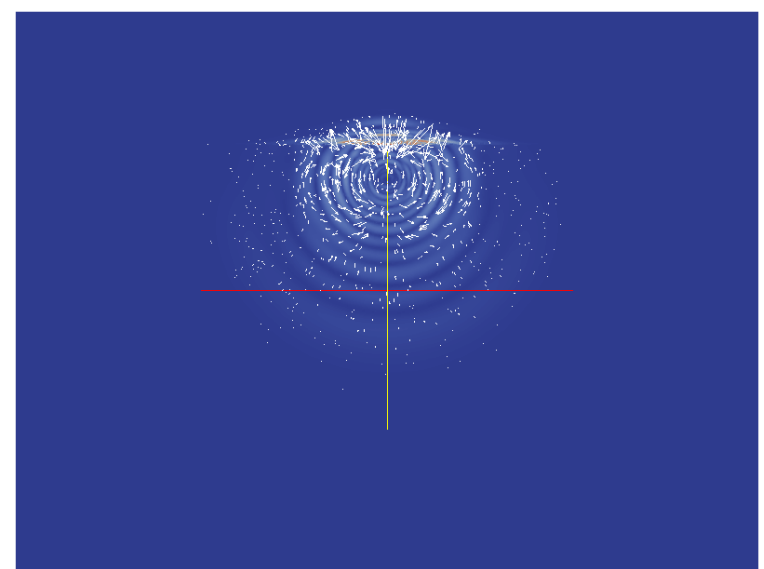

(b) $t=0.016 \mathrm{~s}$

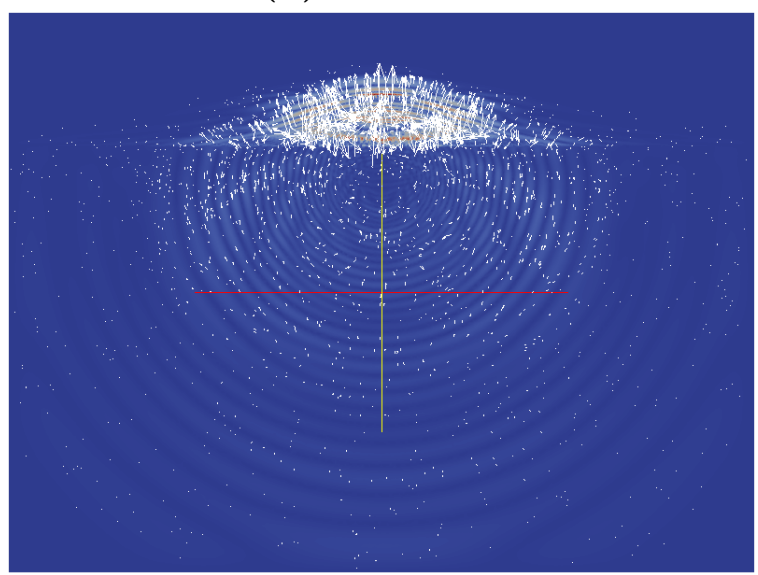

(d) $t=0.041 \mathrm{~s}$

Figure 45: 2nd order PCE coefficient $a_{19}$ for case 2: Magnitude plots (left) and Glyph plots (right) 


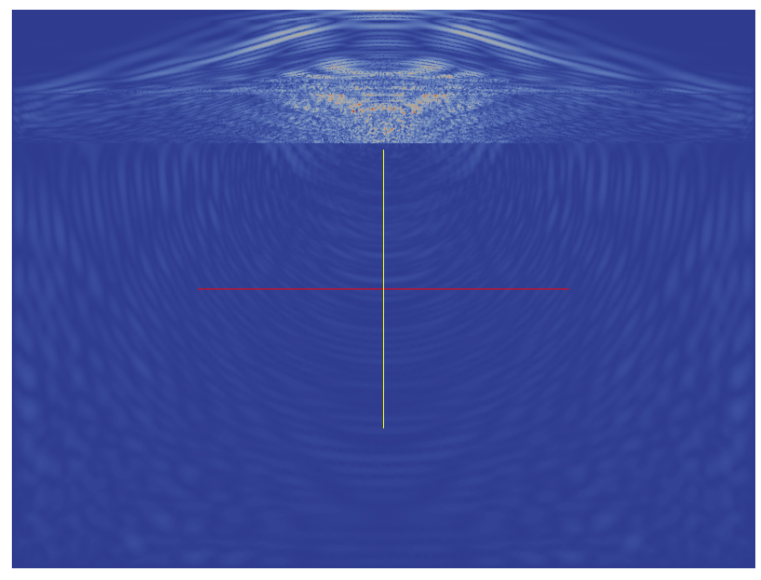

(a) $t=0.096 \mathrm{~s}$

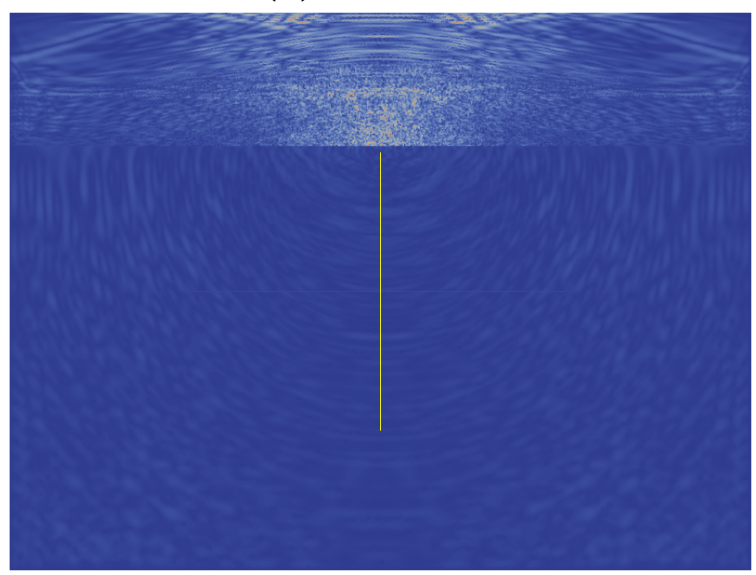

(c) $t=0.136 \mathrm{~s}$

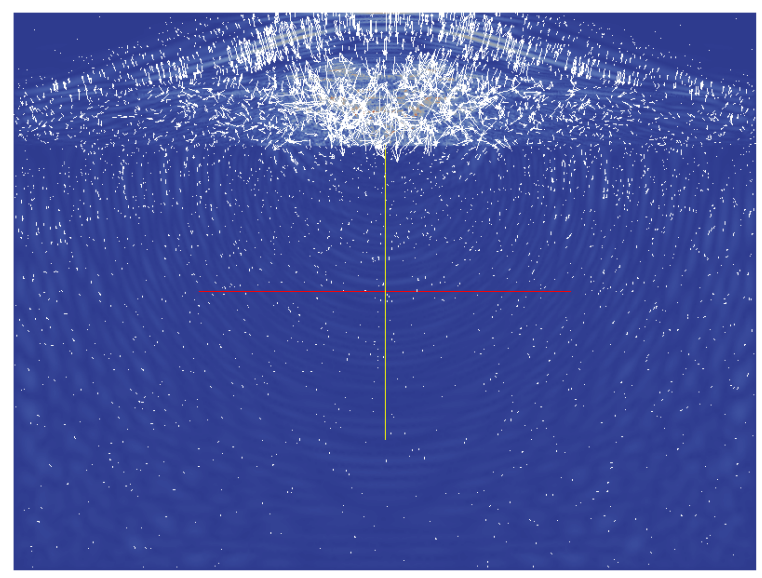

(b) $t=0.096 \mathrm{~s}$

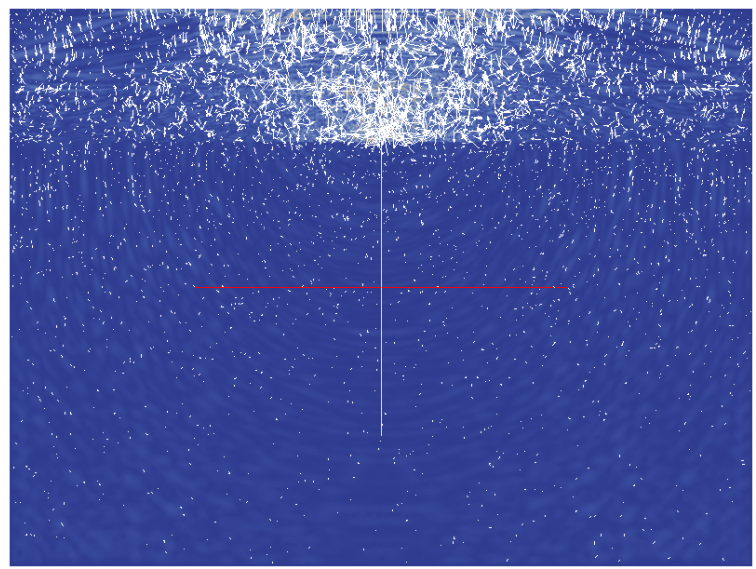

(d) $t=0.136 \mathrm{~s}$

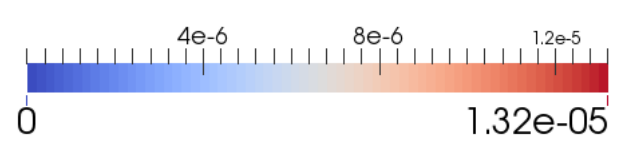

Figure 46: 2nd order PCE coefficient $a_{19}$ for case 2: Magnitude plots (left) and Glyph plots (right) 


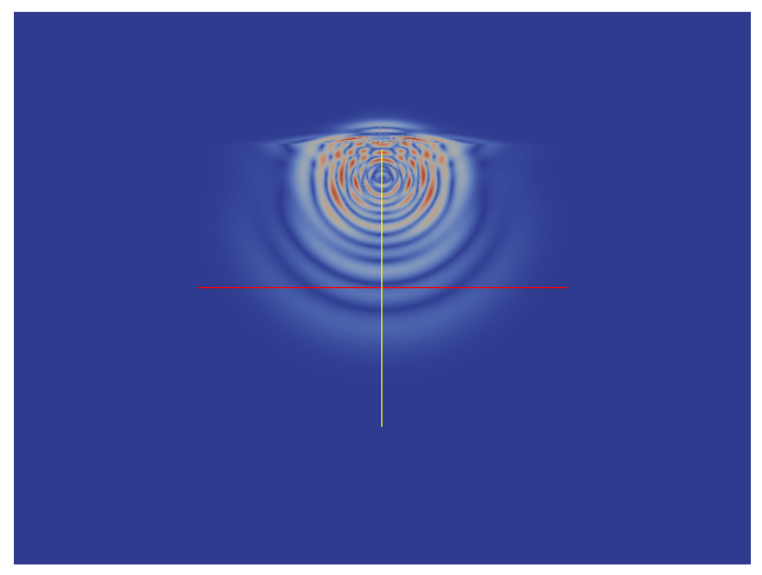

(a) $t=0.016 \mathrm{~s}$

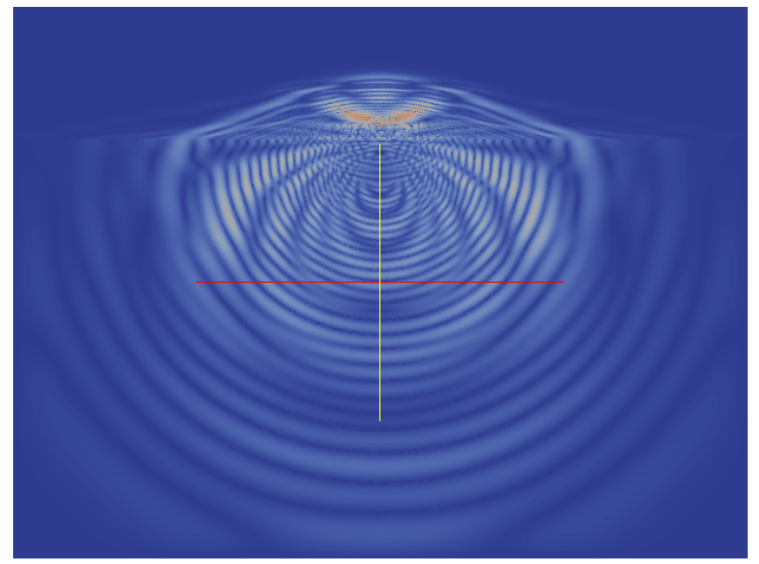

(c) $t=0.041 \mathrm{~s}$

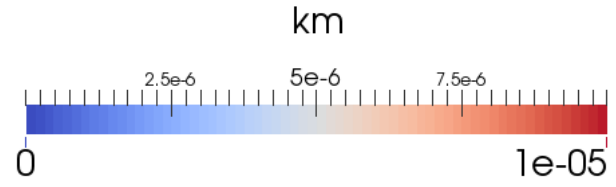

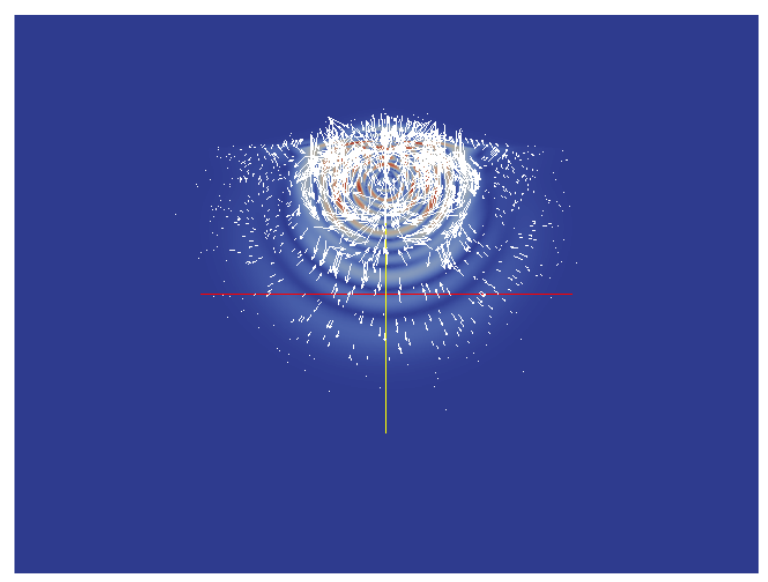

(b) $t=0.016 \mathrm{~s}$

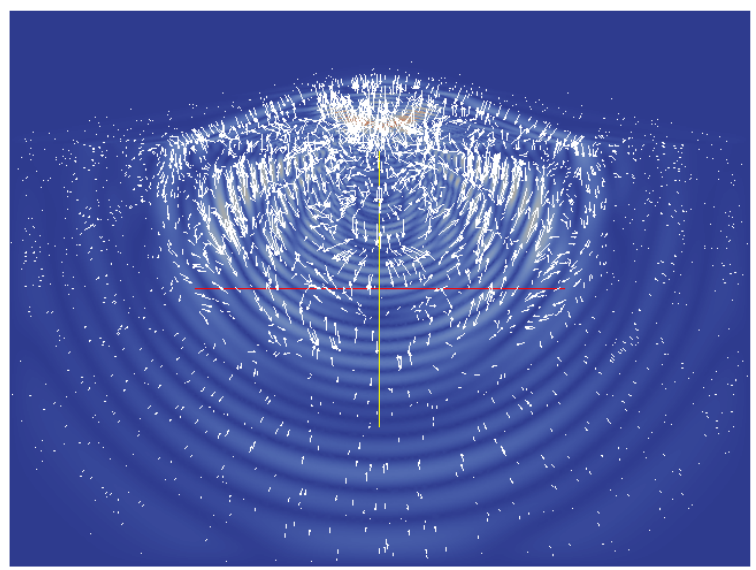

(d) $t=0.041 \mathrm{~s}$

Figure 47: $3 r d$ order PCE coefficient $a_{83}$ for case 2: Magnitude plots (left) and Glyph plots (right) 


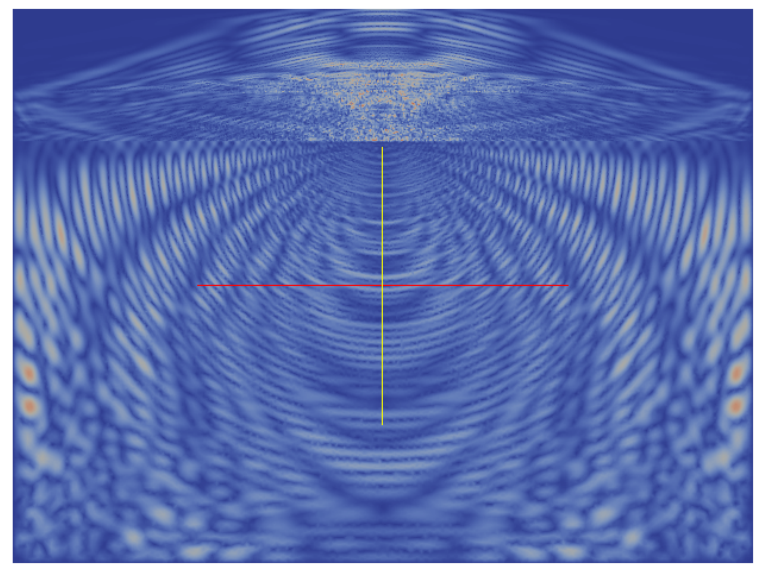

(a) $t=0.096 \mathrm{~s}$

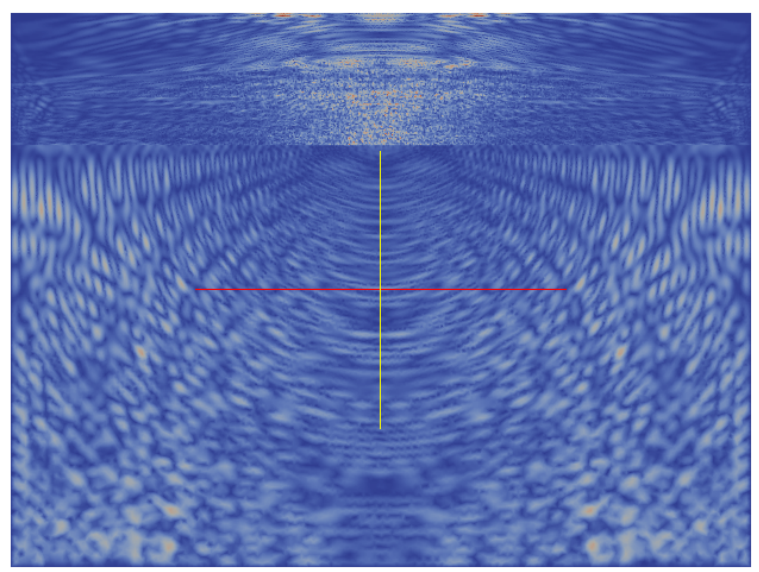

(c) $t=0.136 \mathrm{~s}$

$\mathrm{km}$

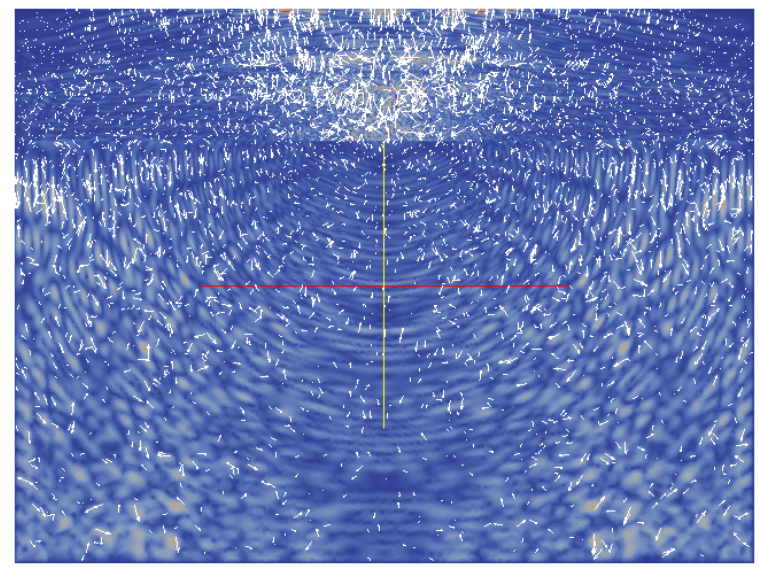

(d) $t=0.136 \mathrm{~s}$

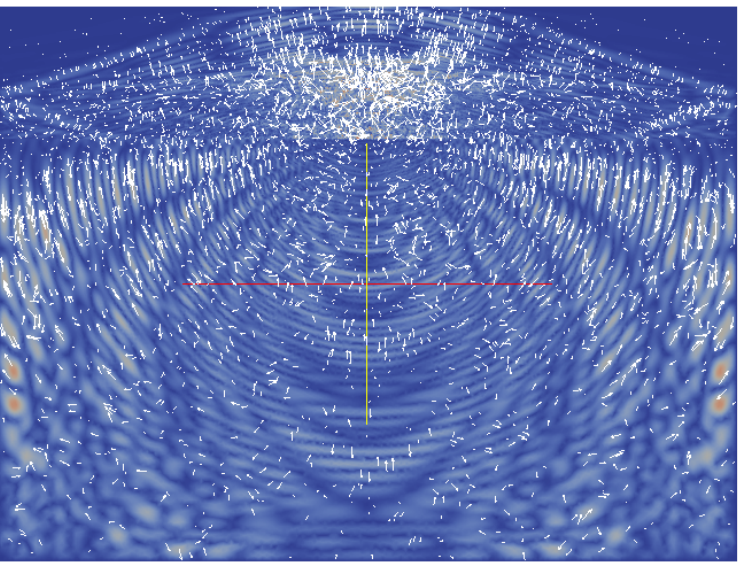

(b) $t=0.096 \mathrm{~s}$

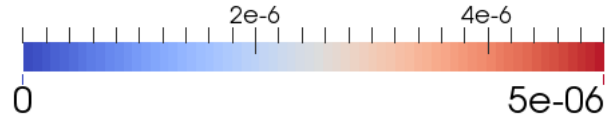

Figure 48: $3 r d$ order PCE coefficient $a_{83}$ for case 2: Magnitude plots (left) and Glyph plots (right) 
observed that for all the time steps, the first PCE coefficient $a_{0}$, i.e., the mean dominates and contributes more than the higher order coefficients which keep decreasing gradually with increasing order.

\begin{tabular}{|l|l|l|}
\hline Parameter & Mean $\mu$ & COV $(\%)$ \\
\hline$E$ & $64 \mathrm{MPa}$ & 40 \\
\hline$\nu$ & 0.3 & - \\
\hline$\rho$ & $1700 \mathrm{~kg} / m^{3}$ & 10 \\
\hline$c$ & $6 \mathrm{kPa}$ & 25 \\
\hline$\phi$ & $23.5^{\circ}$ & 10 \\
\hline$H$ & $0.1 E$ & - \\
\hline$\eta$ & $5 \%$ & - \\
\hline
\end{tabular}

Table 6: Values of Mean and COV for parameters of the Drucker Prager constitutive model

In Fig. 57, the standard deviation has been plotted for three time steps. These plots establish the presence of considerable amount of uncertainty in non-linear wave propagation. Standard deviation in this case looks spatially more scattered compared to the linear elastic case, i.e., the variability is more spread out. This uncertainty decreases with time. 


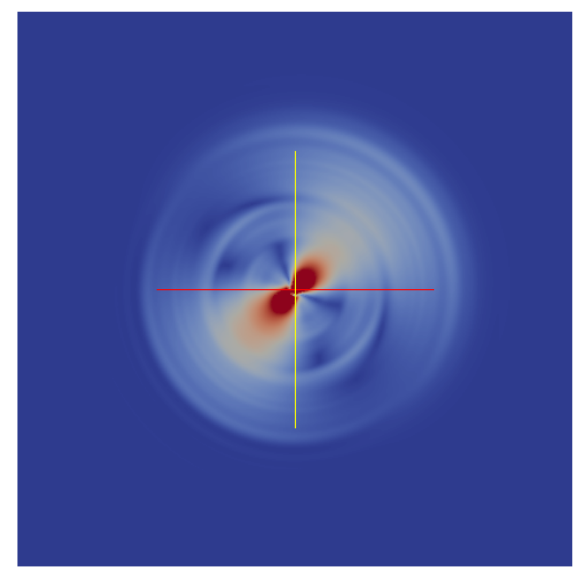

(a) Mean: Magnitude

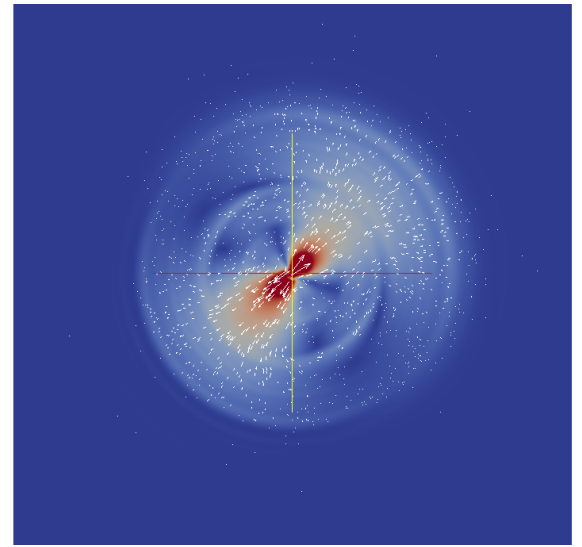

(b) Mean: Glyph

$\mathrm{km}$

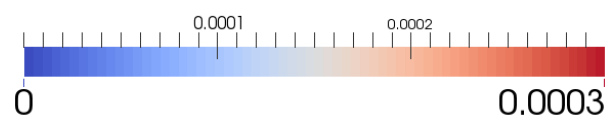

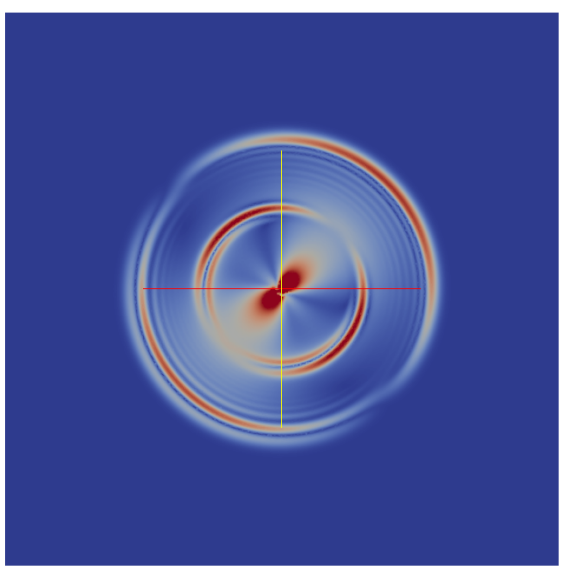

(c) Deterministic: Magnitude

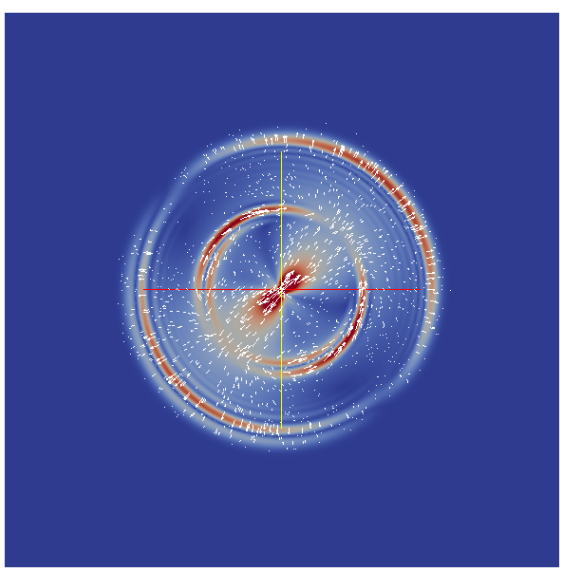

(d) Deterministic: Glyph

$\mathrm{km}$

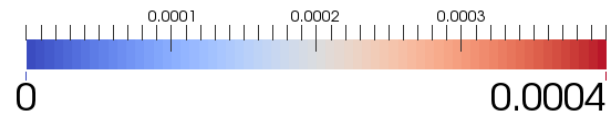

Figure 49: Magnitude of waves propagating in a homogeneous elastoplastic Drucker Prager soil medium: mean $\left(a_{0}\right)$ and deterministic output at $t=1.2 \mathrm{~s}$ 


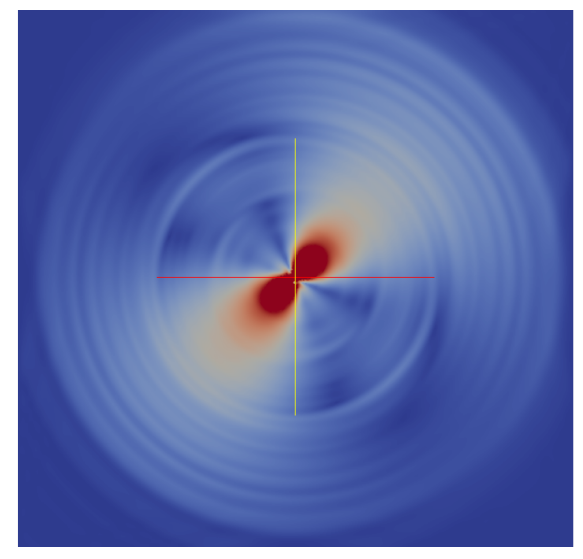

(a) Mean: Magnitude

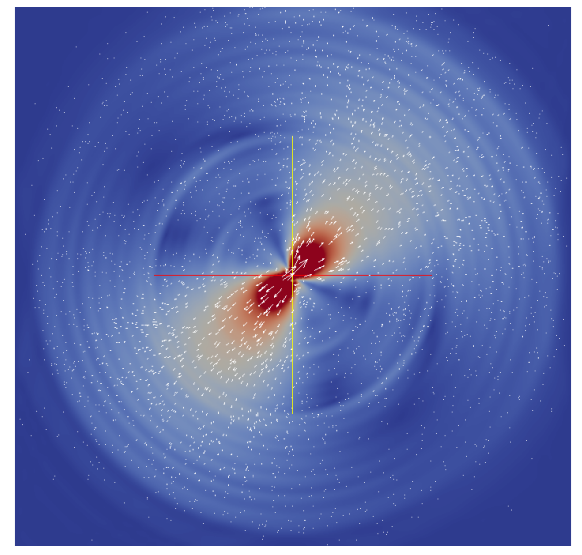

(b) Mean: Glyph

$\mathrm{km}$

0.0001

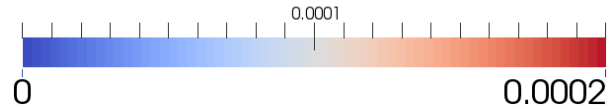

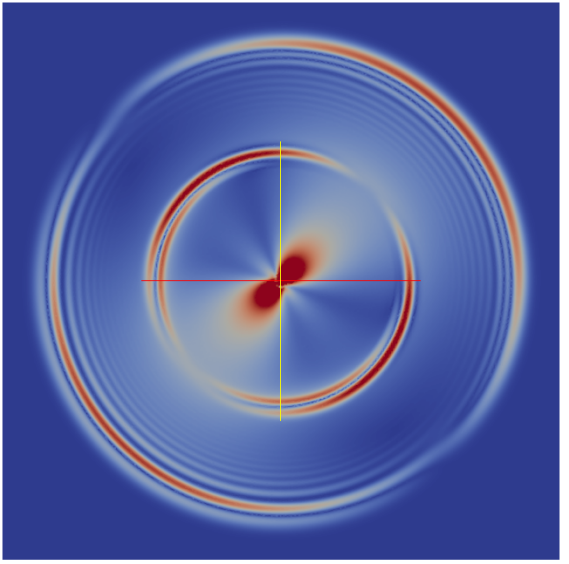

(c) Deterministic: Magnitude

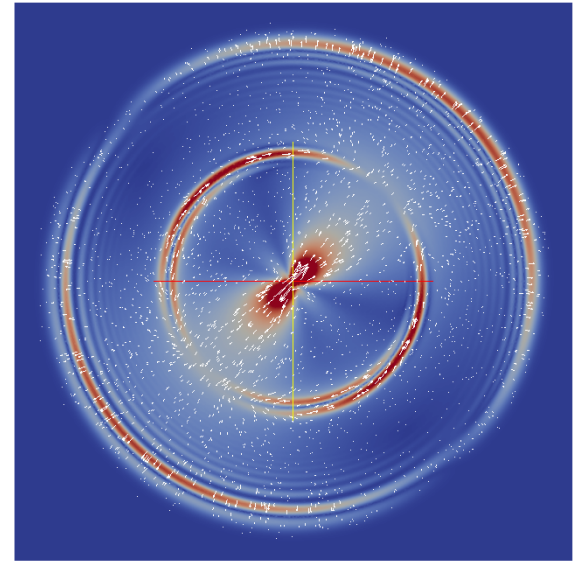

(d) Deterministic: Glyph

$\mathrm{km}$

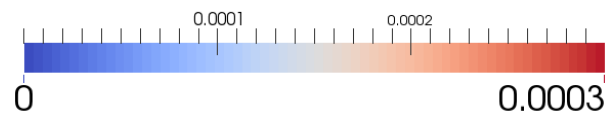

Figure 50: Magnitude of waves propagating in a homogeneous elastoplastic Drucker Prager soil medium: mean $\left(a_{0}\right)$ and deterministic output at $t=1.9 \mathrm{~s}$ 


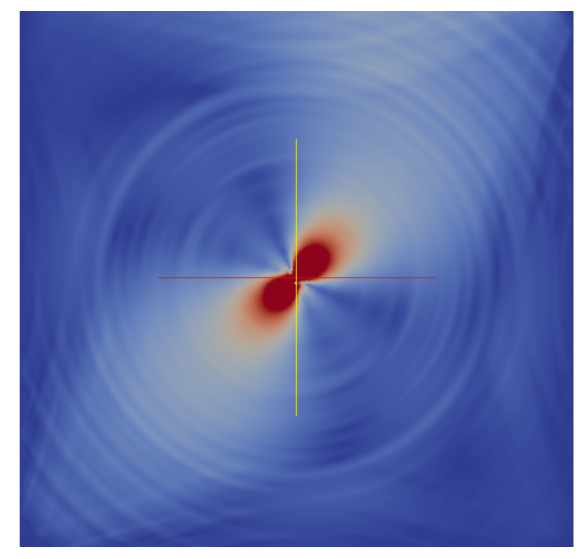

(a) Mean: Magnitude

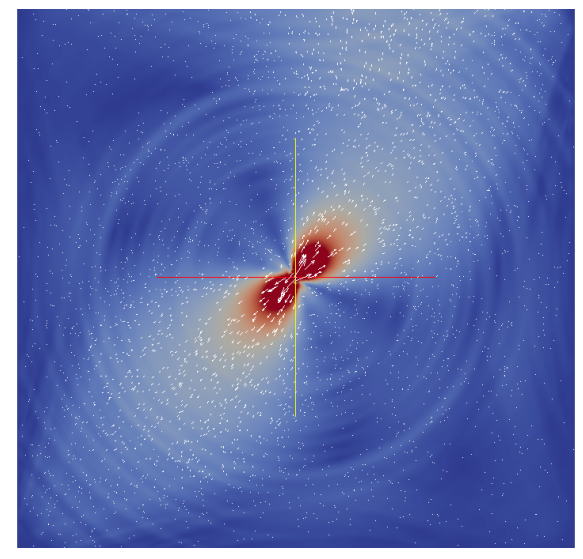

(b) Mean: Glyph

$\mathrm{km}$

0.0001

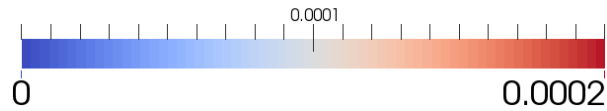

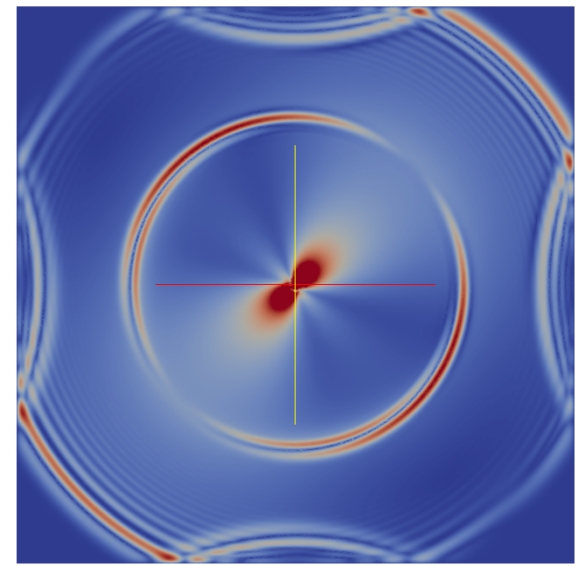

(c) Deterministic: Magnitude

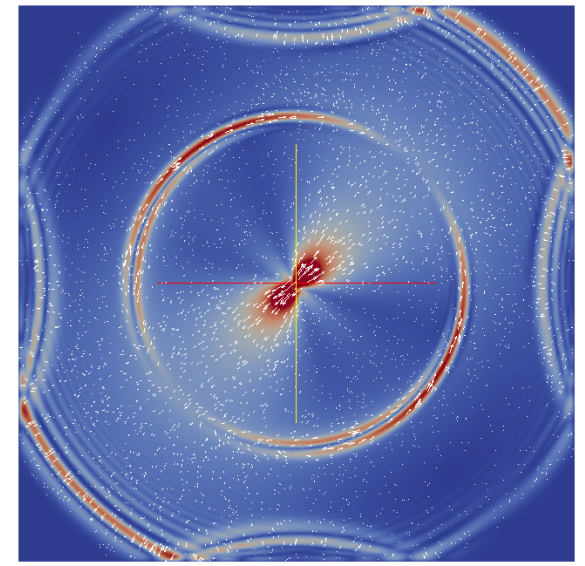

(d) Deterministic: Glyph

$\mathrm{km}$

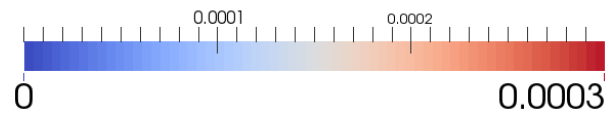

Figure 51: Magnitude of waves propagating in a homogeneous elastoplastic Drucker Prager soil medium: mean $\left(a_{0}\right)$ and deterministic output at $t=2.5 \mathrm{~s}$ 


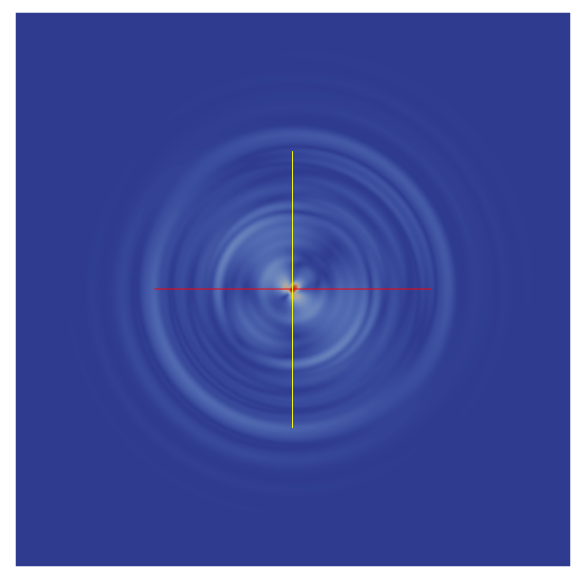

(a) $t=1.2 \mathrm{~s}$

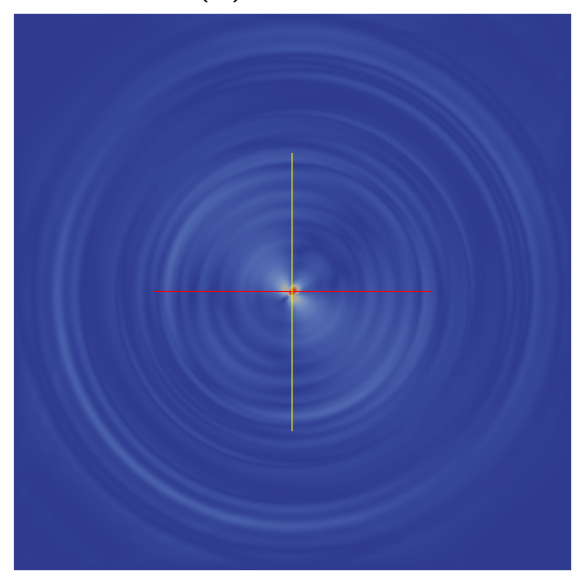

(c) $t=1.9 \mathrm{~s}$

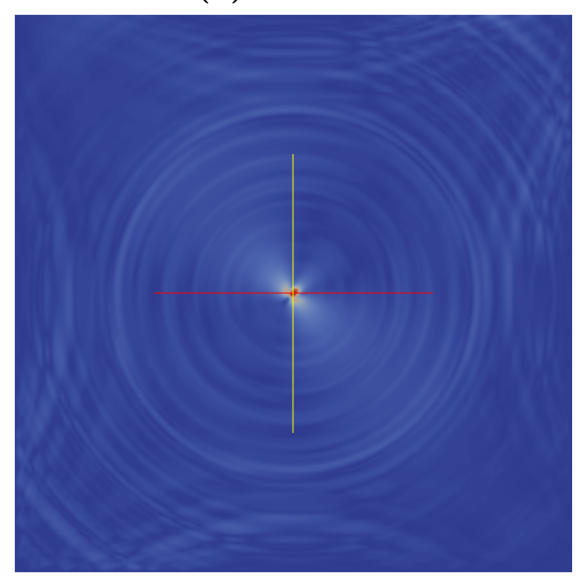

(e) $t=2.5 \mathrm{~s}$

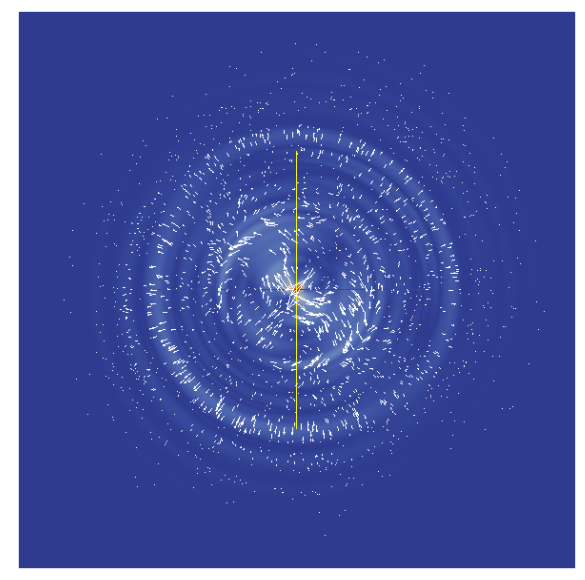

(b) $t=1.2 \mathrm{~s}$

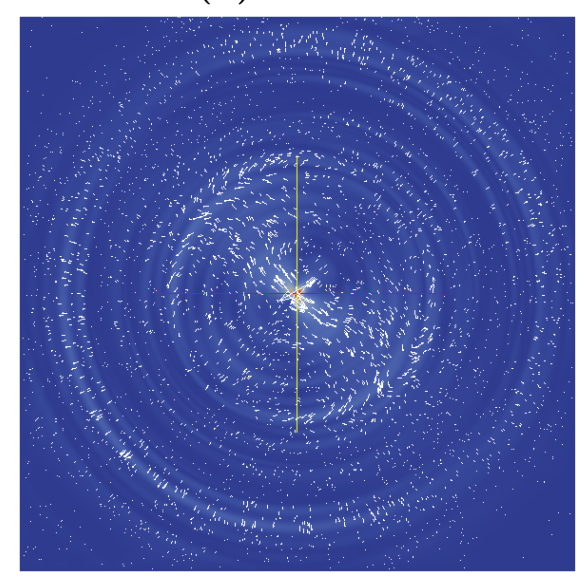

(d) $t=1.9 \mathrm{~s}$

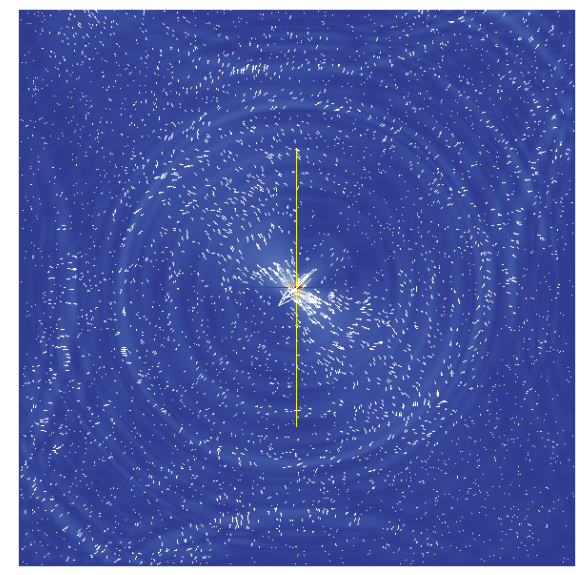

(f) $t=2.5 \mathrm{~s}$

$\mathrm{km}$

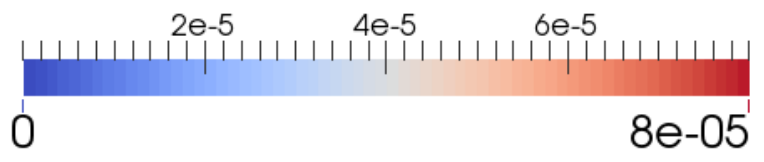

Figure 52: 1 st order PCE coefficient $a_{3}$ for case 3: Magnitude plots (left) and Glyph plots (right) 


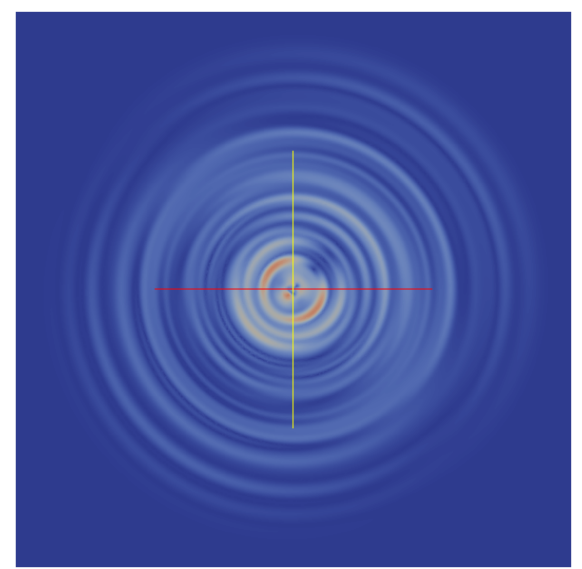

(a) $t=1.2 \mathrm{~s}$

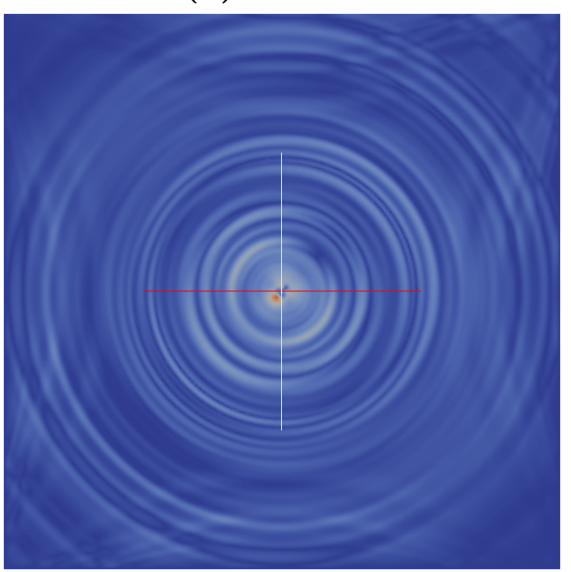

(c) $t=1.9 \mathrm{~s}$

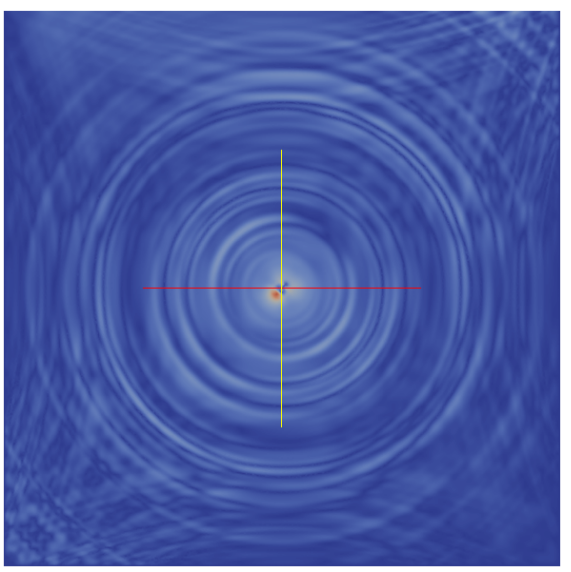

(e) $t=2.5 \mathrm{~s}$

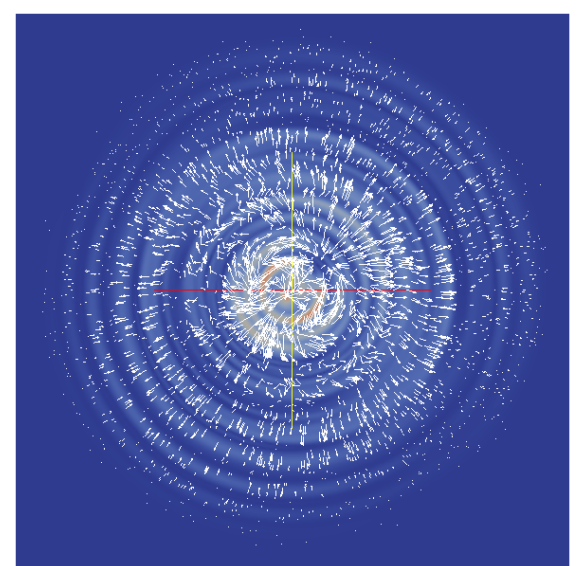

(b) $t=1.2 \mathrm{~s}$

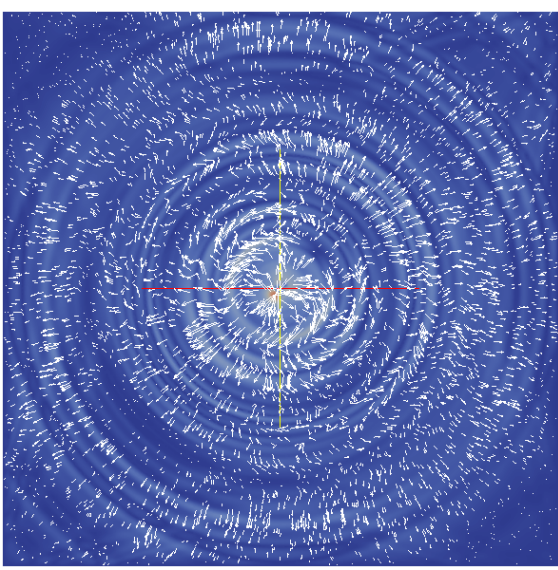

(d) $t=1.9 \mathrm{~s}$

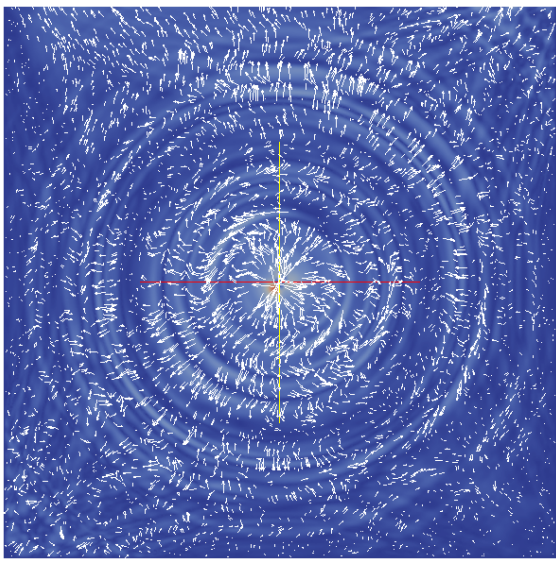

(f) $t=2.5 \mathrm{~s}$

$\mathrm{km}$

$1 e-5$

$2 e-5$

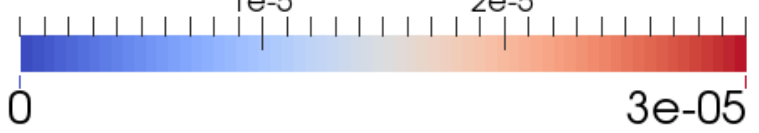

Figure 53: 2nd order PCE coefficient $a_{7}$ for case 3: Magnitude plots (left) and Glyph plots (right) 


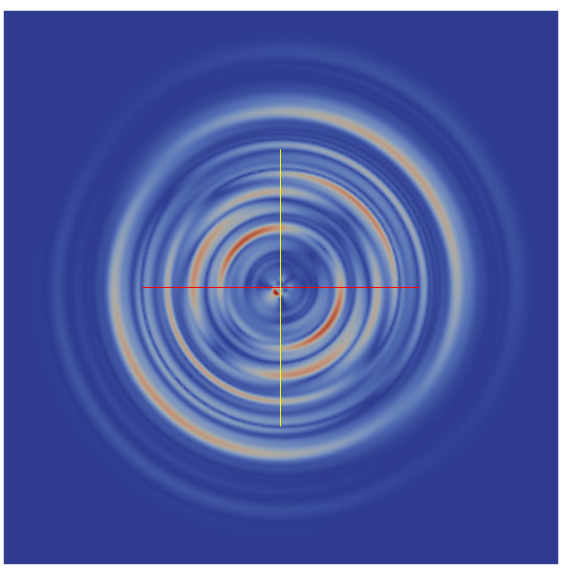

(a) $t=1.2 \mathrm{~s}$

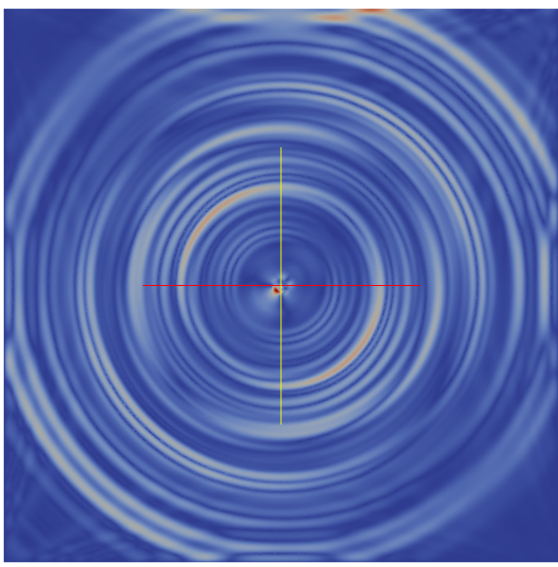

(c) $t=1.9 \mathrm{~s}$

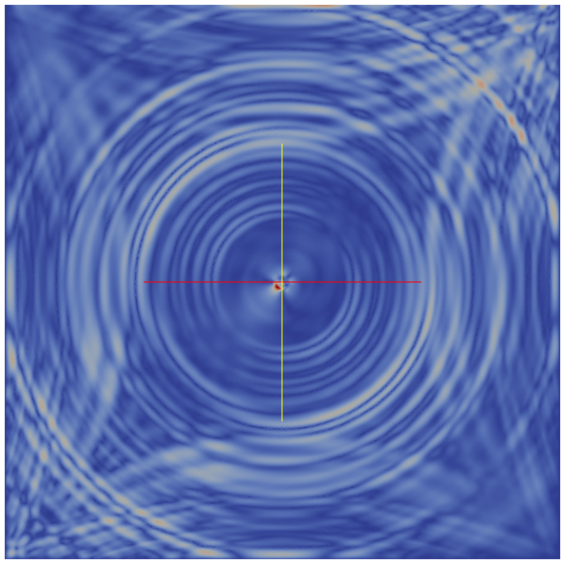

(e) $t=2.5 \mathrm{~s}$

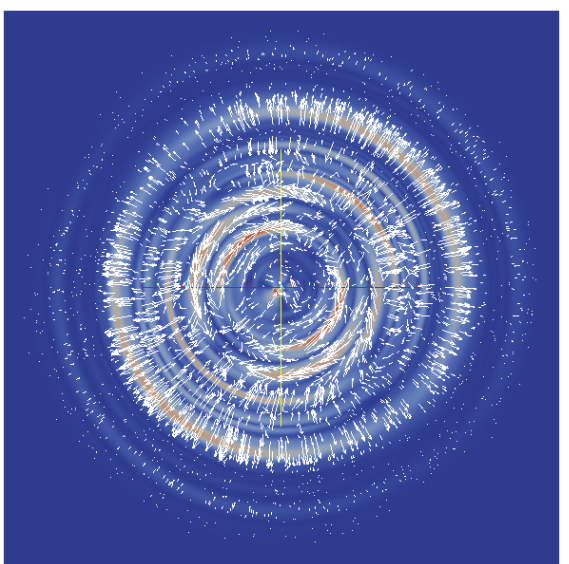

(b) $t=1.2 \mathrm{~s}$

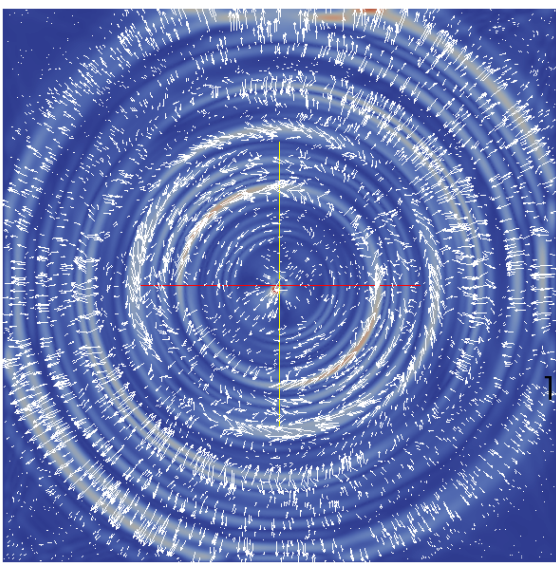

(d) $t=1.9 \mathrm{~s}$

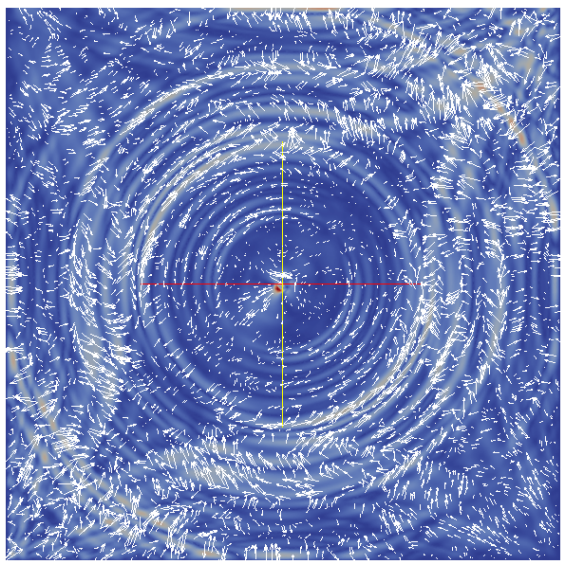

(f) $t=2.5 \mathrm{~s}$

$\mathrm{km}$

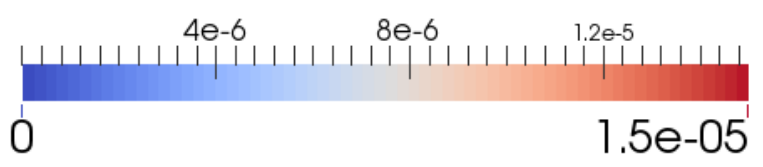

Figure 54: 2nd order PCE coefficient $a_{11}$ for case 3: Magnitude plots (left) and Glyph plots (right) 


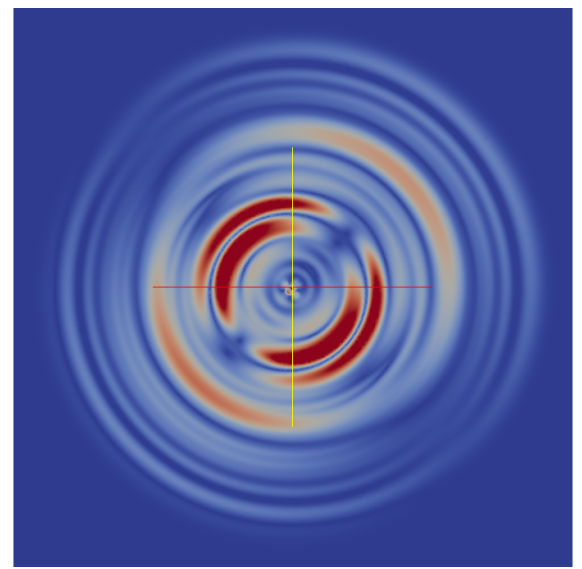

(a) $t=1.2 \mathrm{~s}$

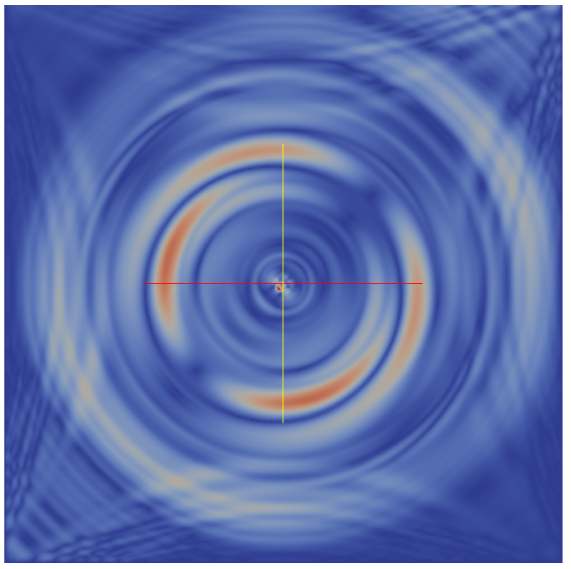

(c) $t=1.9 \mathrm{~s}$

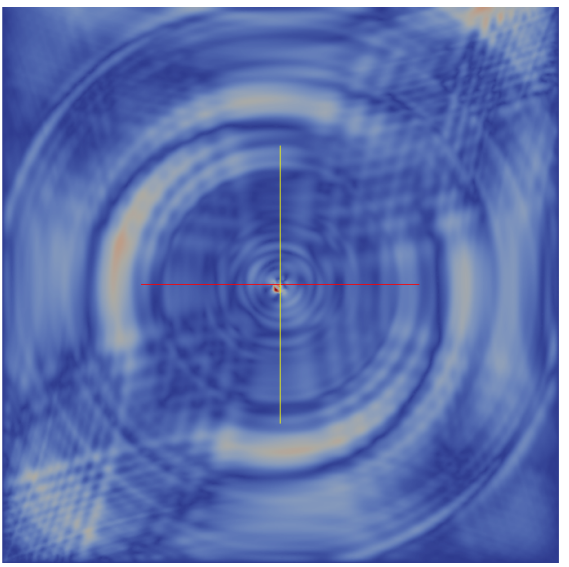

(e) $t=2.5 \mathrm{~s}$

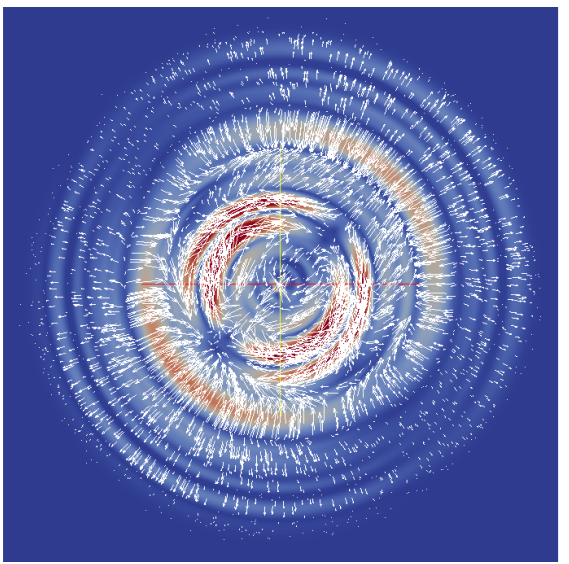

(b) $t=1.2 \mathrm{~s}$

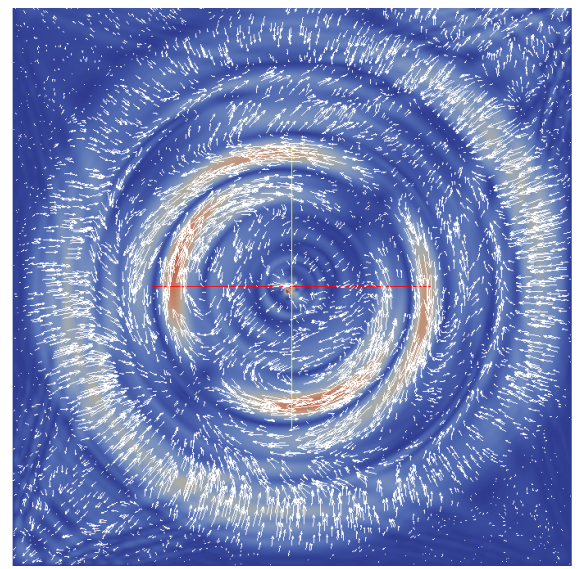

(d) $t=1.9 \mathrm{~s}$

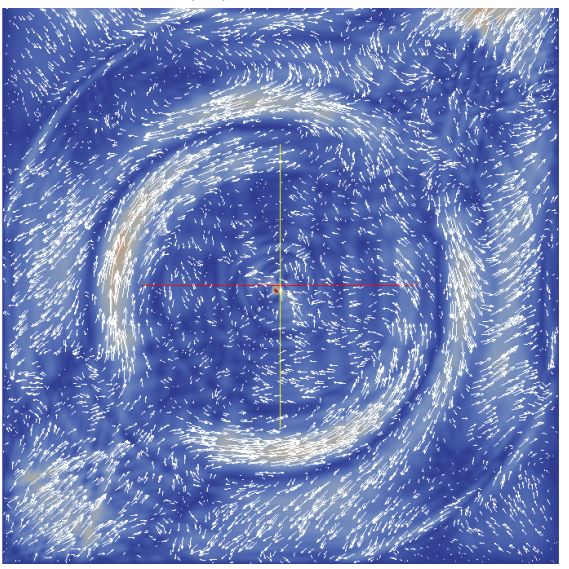

(f) $t=2.5 \mathrm{~s}$

$\mathrm{km}$

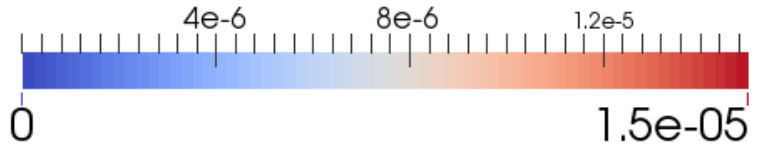

Figure 55: $3 r d$ order PCE coefficient $a_{15}$ for case 3: Magnitude plots (left) and Glyph plots (right) 


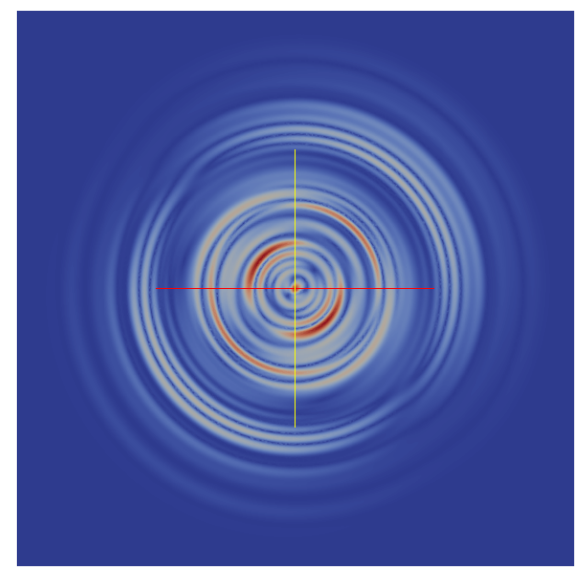

(a) $t=1.2 \mathrm{~s}$

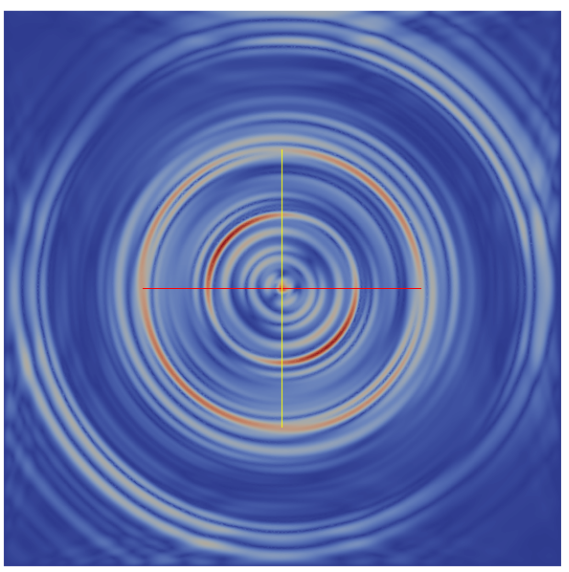

(c) $t=1.9 \mathrm{~s}$

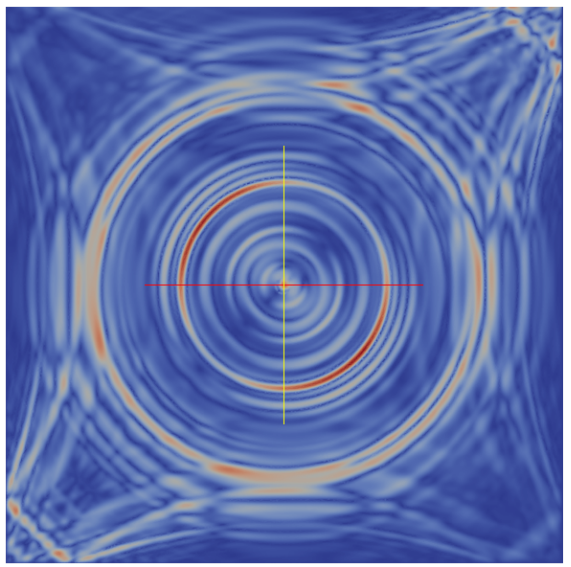

(e) $t=2.5 \mathrm{~s}$

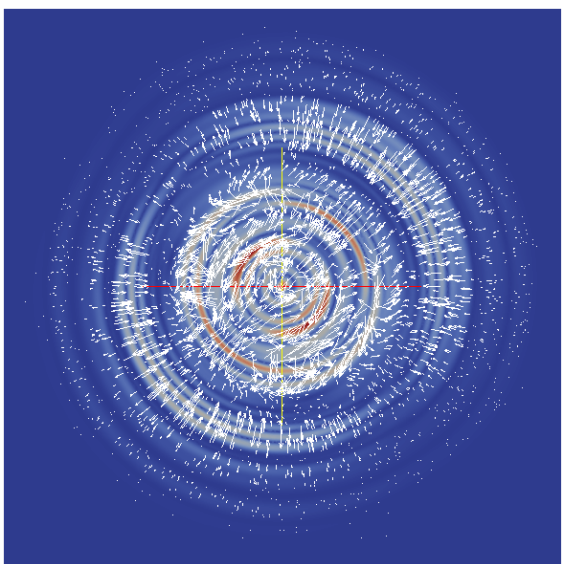

(b) $t=1.2 \mathrm{~s}$

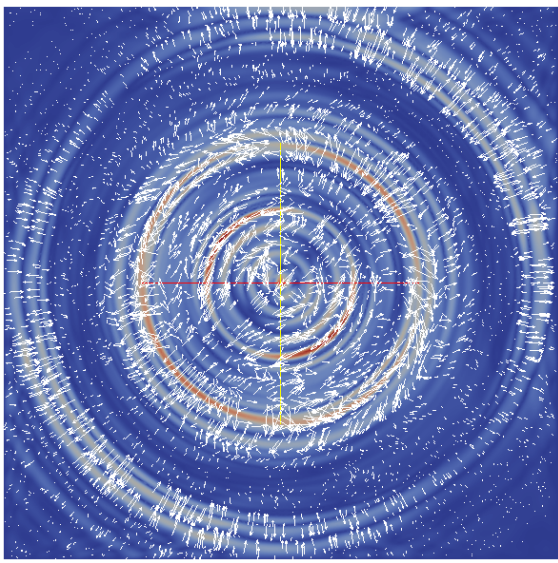

(d) $t=1.9 \mathrm{~s}$

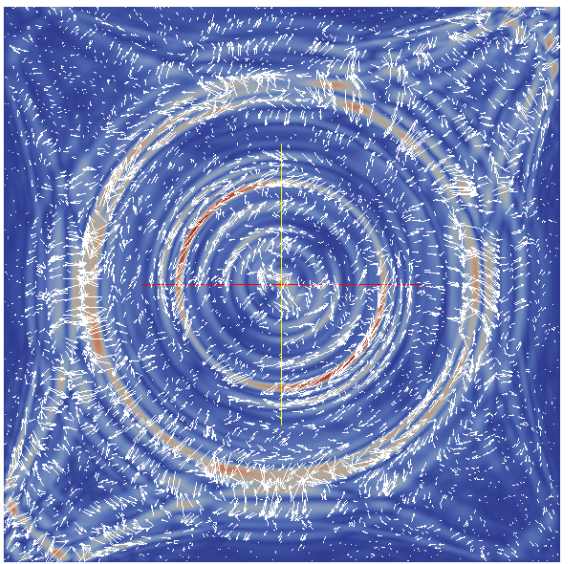

(f) $t=2.5 \mathrm{~s}$

$\mathrm{km}$

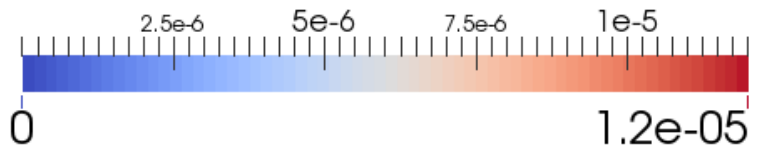

Figure 56: $3 r d$ order PCE coefficient $a_{22}$ for case 3: Magnitude plots (left) and Glyph plots (right) 


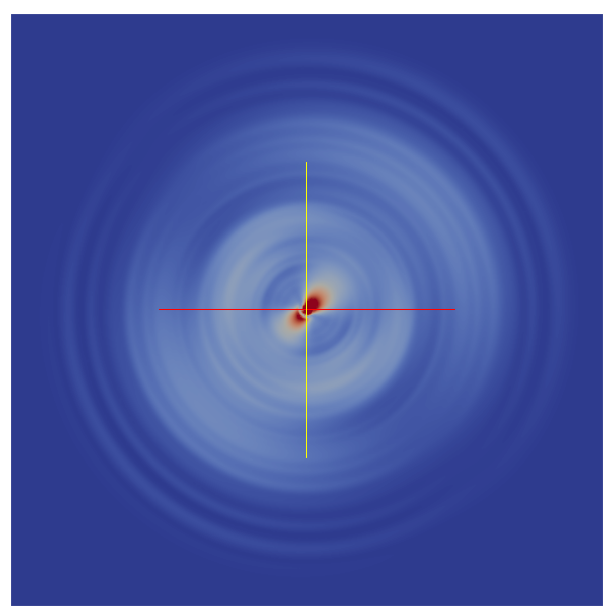

(a) $t=1.2 \mathrm{~s}$

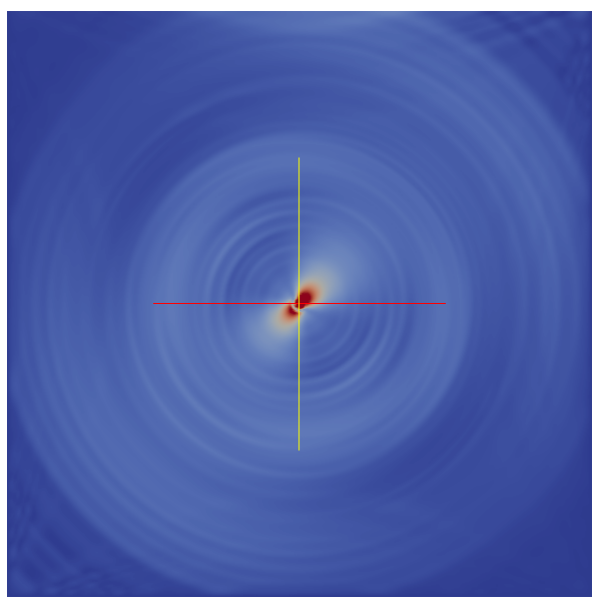

(b) $t=1.9 \mathrm{~s}$

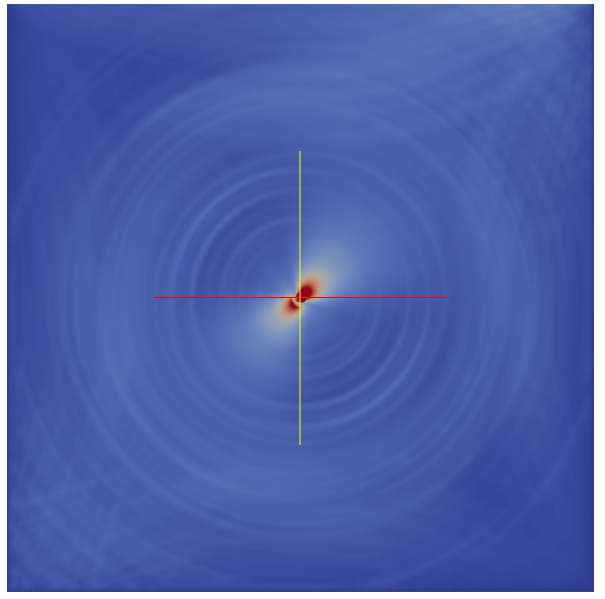

(c) $t=2.5 \mathrm{~s}$

$\mathrm{km}$

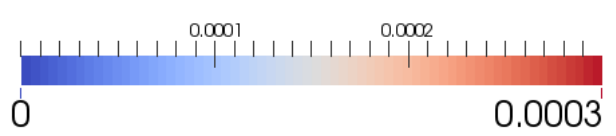

Figure 57: Standard deviation for the elasto-plastic model (4 random variables and $3 r d$ order expansion) 


\section{Chapter 5}

\section{Numerical Application: Results}

\subsection{Concluding Remarks}

In this thesis, a computational framework is developed for the uncertainty quantification in wave propagation, considering two different material models: 1) a linear elastic model, 2) a rate independent associative elasto-plastic Drucker-Prager model. A detailed formulation is provided for solving the problem of wave propagation using finite element method implemented using open source FEniCS solver. A numerically stable, implicit Newmark Beta method is implemented for time integration scheme in FEniCS. Both the linear and non-linear models were tested and validated with published results. The capability of the computational framework to perform largescale simulations is demonstrated by tackling a high resolution model (with over 100 million dof) using 1040 cores with 65 nodes, and 92 GB of memory per node. While results were presented only for 2-D simulations, it was also shown how the model can be conveniently extended to solve a 3D dynamic plasticity problem. When compared with the linear reference simulation in general, the non-linear simulations displayed noticeable permanent displacement and more spatial variation in response, which is in agreement with the findings in the literature [133]. 
Next, uncertainty quantification based on a non-intrusive polynomial chaos method (PCE) is conducted for three different cases: 1) a linear elastic 2-D homogeneous soil medium, (2) a linear elastic 2-D layered piecewise homogeneous soil profile, (3) a non-linear elasto-plastic 2-D homogeneous soil medium. In the resulting contours, a noticeable departure was observed in the behaviour of probabilistic mean response from the deterministic solution. Also, considerable scatter is observed in the standard deviation plots with respect to the mean which indicates that the COV in output is quite high and this justifies the need for uncertainty quantification in both linear and non-linear seismic wave propagation.

The specific contributions of this work can be further summarized as follows. In the past, quite a few studies have focussed on modelling elasto-plastic waves in soil media. However, most of these codes are closed source, and have been developed using expensive commercial software, or tools that are restricted to certain research groups and not readily available in public domain. The elasto-plastic wave propagation model developed in this thesis is based on a state-of-the-art finite element based PDE solver called FEniCS, which exploits scalable parallel numerical libraries for high performance computing. The meshes are generated using the free Gmsh tool and visualisations are done in ParaView. To the best of our knowledge, no other computational elasto-plastic wave propagation solver built on open source libraries has demonstrated parallel processing capabilities for high resolution numerical model which resolves a wide range of frequencies in non-linear seismic wave propagation. Solving such large-scale problems with the use of commercial software will also turn out to be very expensive.

Moving to the problem of uncertainty quantification, most of the relevant studies performed to date have focused only on linear elastic media and Monte Carlo sampling. Those which have dealt with elasto-plastic models, have been restricted mostly 
to 1-D simulations and have only implemented the intrusive method which is difficult to implement. On the other hand, we have used a convenient non-intrusive polynomial chaos method which can readily exploit the deterministic code for uncertainty quantification.

\section{$5.2 \quad$ Future Research}

The computational framework developed in this thesis is a 2-D plane strain model for isotropic plasticity based on Drucker Prager yield criterion with isotropic hardening. In reality though, soils are heterogeneous and isotropy is therefore not a good assumption. However, it allows for simple explanation of the basic concepts, which can be further refined and applied to more complicated problems [43].

The author believes that the model should be extended to include the effects of multiple layers and heterogeneity in a three dimensional framework which also considers kinematic hardening. Also, in the current work, the uncertain physical parameters were treated as random variables. In a future work, it would be interesting to see how the probabilistic response changes when these parameters are treated as a random process. The feasibility of implementing an intrusive approach for uncertainty quantification should also be investigated now that we have already implemented a non-intrusive approach. Additional work is also required to modify the model such that it can take realistic input source (based on previous earthquake data) into account along with absorbing boundary conditions and a more suitable type of damping. It would then be of significant interest to compare the results with existing popular software designed for ground motion simulation. 


\section{Bibliography}

[1] D. Xiu and G. E. Karniadakis. "The wiener-askey polynomial chaos for stochastic differential equations." SIAM journal on scientific computing 24(2), 619-644 (2002).

[2] L. Fagiano and M. Khammash. "Simulation of stochastic systems via polynomial chaos expansions and convex optimization." Physical Review E 86(3), $036702(2012)$.

[3] O. C. Zienkiewicz and R. L. Taylor. The finite element method for solid and structural mechanics. Butterworth heinemann (2005).

[4] K. Krabbenhøft. "Basic computational plasticity." Lecture Notes (2002).

[5] P. Kelly. "Engineering solid mechanics." Part 2, 301-313 (2008).

[6] O. Le Maître and O. M. Knio. Spectral methods for uncertainty quantification: with applications to computational fluid dynamics. Springer Science \& Business Media (2010).

[7] J. Bielak, O. Ghattas, and E. Kim. "Parallel octree-based finite element method for large-scale earthquake ground motion simulation." Computer Modeling in Engineering and Sciences 10(2), 99 (2005).

[8] B. Jeremić, K. Sett, and M. L. Kavvas. "Probabilistic elasto-plasticity: formulation in 1d." Acta Geotechnica 2(3), 197-210 (2007).

[9] K. Sett, K. Eshun, Y. C. Chao, and B. Jeremić. "Effect of uncertain spatial variability of soils on nonlinear seismic site response analysis." In "GeoCongress 
2012: State of the Art and Practice in Geotechnical Engineering," pages 28562865. ASCE (2012).

[10] P. M. Shearer. Introduction to seismology. Cambridge University Press (2009).

[11] G. T. Schuster. "Basics of seismic wave theory." de Notes for the Lecture Courses, University of Utah (2007).

[12] J. d. D. De Basabe Delgado. "High-order finite element methods for seismic wave propagation." (2009).

[13] S. Mishra, C. Schwab, and J. Šukys. "Multi-level monte carlo finite volume methods for uncertainty quantification of acoustic wave propagation in random heterogeneous layered medium." Journal of Computational Physics 312, 192$217(2016)$.

[14] J. Bielak, J. Xu, and O. Ghattas. "Earthquake ground motion and structural response in alluvial valleys." Journal of Geotechnical and Geoenvironmental Engineering 125(5), 413-423 (1999).

[15] C. Yeh and M. Rahman. "Stochastic finite element methods for the seismic response of soils." International journal for numerical and analytical methods in geomechanics 22(10), 819-850 (1998).

[16] J. Xu. Three-dimensional simulation of wave propagation in inelastic media on parallel computers. Ph.D. thesis, Carnegie Mellon University (1998).

[17] T. Furumura and L. Chen. "Parallel simulation of strong ground motions during recent and historical damaging earthquakes in tokyo, japan." Parallel Computing 31(2), 149-165 (2005). 
[18] H. Bao, J. Bielak, O. Ghattas, L. F. Kallivokas, D. R. O'Hallaron, J. R. Shewchuk, and J. Xu. "Large-scale simulation of elastic wave propagation in heterogeneous media on parallel computers." Computer methods in applied mechanics and engineering 152(1), 85-102 (1998).

[19] K. S. Yee et al. "Numerical solution of initial boundary value problems involving maxwells equations in isotropic media." IEEE Trans. Antennas Propag 14(3), 302-307 (1966).

[20] J. E. Kozdon, E. M. Dunham, and J. Nordström. "Simulation of dynamic earthquake ruptures in complex geometries using high-order finite difference methods." Journal of Scientific Computing 55(1), 92-124 (2013).

[21] R. W. Graves. "Simulating seismic wave propagation in 3d elastic media using staggered-grid finite differences." Bulletin of the Seismological Society of America 86(4), 1091-1106 (1996).

[22] V. Bokil. Computational methods for wave propagation problems in unbounded domains. Ph.D. thesis, University of Houston (2003).

[23] M. Dumbser, M. Käser, and J. De La Puente. "Arbitrary high-order finite volume schemes for seismic wave propagation on unstructured meshes in $2 \mathrm{~d}$ and 3d." Geophysical Journal International 171(2), 665-694 (2007).

[24] L. Zhang and A. K. Chopra. "Three-dimensional analysis of spatially varying ground motions around a uniform canyon in a homogeneous half-space." Earthquake engineering 6 structural dynamics 20(10), 911-926 (1991). 
[25] F. Dupros, F. De Martin, E. Foerster, D. Komatitsch, and J. Roman. "Highperformance finite-element simulations of seismic wave propagation in threedimensional nonlinear inelastic geological media." Parallel Computing 36(5), 308-325 (2010).

[26] V. Akcelik, J. Bielak, G. Biros, I. Epanomeritakis, A. Fernandez, O. Ghattas, E. J. Kim, J. Lopez, D. O'Hallaron, T. Tu, et al. "High resolution forward and inverse earthquake modeling on terascale computers." In "Supercomputing, 2003 ACM/IEEE Conference," pages 52-52. IEEE (2003).

[27] H. Zheng, Z. Zhang, and E. Liu. "Non-linear seismic wave propagation in anisotropic media using the flux-corrected transport technique." Geophysical Journal International 165(3), 943-956 (2006).

[28] J. Xu, J. Bielak, O. Ghattas, and J. Wang. "Three-dimensional nonlinear seismic ground motion modeling in basins." Physics of the earth and planetary interiors 137(1), 81-95 (2003).

[29] V. Streeter, E. Wylie, and F. Richart. "Soil motion computations by characteristics method: 12f, 16r. j. geotech. engng. div. v100, n. gt3, mar. 1974, p247-263." In "International Journal of Rock Mechanics and Mining Sciences \& Geomechanics Abstracts," volume 11, page A164. Pergamon (1974).

[30] W. Liam Finn, G. Martin, and M. Lee. "Comparison of dynamic analyses for saturated sands." In "From Volume I of Earthquake Engineering and Soil Dynamics-Proceedings of the ASCE Geotechnical Engineering Division Specialty Conference, June 19-21, 1978, Pasadena, California. Sponsored by Geotechnical Engineering Division of ASCE in cooperation with:," Proceeding (1978). 
[31] E. Foerster and H. Modaressi. "Nonlinear numerical method for earthquake site response analysis iicase studies." Bulletin of Earthquake Engineering 5(3), 325-345 (2007).

[32] P. B. Schnabel. "Shake a computer program for earthquake response analysisi of horizontally layered sites." EERC report pages 72-12 (1972).

[33] I. M. Idriss and H. B. Seed. "Seismic response of horizontal soil lauers." Am Soc Civil Engr J Soil Mech (1968).

[34] B. O. Hardin and V. P. Drnevich. "Shear modulus and damping in soils: measurement and parameter effects." Journal of Soil Mechanics $\mathcal{E}$ Foundations Div 98(sm6) (1972).

[35] M. Vucetic and R. Dobry. "Effect of soil plasticity on cyclic response." Journal of geotechnical engineering 117(1), 89-107 (1991).

[36] H. B. Seed and I. M. Idriss. "Soil moduli and damping factors for dynamic response analyses." (1970).

[37] W. Finn. "Geotechnical engineering aspects of microzonation." In "Proc. 4th International Conference on Seismic Zonation," volume 1, pages 199-259 (1991).

[38] H. B. Seed and I. M. Idriss. Influence of soil conditions on ground motions during earthquakes. University of California, Institute of Transportation and Traffic Engineering, Soil Mechanics Laboratory (1969).

[39] C. Di Prisco, S. Imposimato, and E. Aifantis. "A visco-plastic constitutive model for granular soils modified according to non-local and gradient approaches." International journal for numerical and analytical methods in geomechanics 26(2), 121-138 (2002). 
[40] M. Mabssout and M. Pastor. "A taylor-galerkin algorithm for shock wave propagation and strain localization failure of viscoplastic continua." Computer Methods in Applied Mechanics and Engineering 192(7), 955-971 (2003).

[41] M. Stupazzini and C. Zambelli. "Geo-elsevp: a spectral element approach for 2d or 3d dynamic elasto-viscoplastic problems." Rivista Italiana di Geotecnica 39(4), 70-82 (2005).

[42] H. G. Matthies. "Uncertainty quantification with stochastic finite elements." Encyclopedia of computational mechanics (2007).

[43] B. Rosić and H. Matthies. "Computational approaches to inelastic media with uncertain parameters." Journal of the Serbian Society for Computational Mechanics 2(1), 28-43 (2008).

[44] K. Sett and B. Jeremic. "Seismic wave propagation in stochastic soil." In "EERIs Eight US National Conference on Earthquake Engineering, San Francisco, CA," 519 (2006).

[45] M. Rahman and C. Yeh. "Variability of seismic response of soils using stochastic finite element method." Soil Dynamics and Earthquake Engineering 18(3), 229245 (1999).

[46] A. Nour, A. Slimani, N. Laouami, and H. Afra. "Finite element model for the probabilistic seismic response of heterogeneous soil profile." Soil dynamics and earthquake engineering 23(5), 331-348 (2003).

[47] A. FLUSHPLUS. "computer program for approximate 3-d analysis of soilstructure interaction problems." An enhanced PC version of the original FLUSH program published in (1975). 
[48] K. Haciefendioğlu, A. Bayraktar, and H. B. Başağa. "Estimation of stochastic nonlinear dynamic response of rock-fill dams with uncertain material parameters for non-stationary random seismic excitation." Nonlinear Dynamics 61(1-2), 43-55 (2010).

[49] B. Jeremic and K. Sett. "Uncertain soil properties and elastic-plastic simulations in geomechanics." In "Geo-Denver 2007 Congress: New Peaks in Geotechnics," pages 1-11 (2007).

[50] S. Acharjee and N. Zabaras. "Uncertainty propagation in finite deformations-a spectral stochastic lagrangian approach." Computer methods in applied mechanics and engineering 195(19), 2289-2312 (2006).

[51] R. L. Iman. "Latin hypercube sampling." Encyclopedia of quantitative risk analysis and assessment (2008).

[52] M. Giles. "Improved multilevel monte carlo convergence using the milstein scheme." In "Monte Carlo and quasi-Monte Carlo methods 2006," pages 343358. Springer (2008).

[53] R. J. AQUINO. "Stochastic finite element modeling and its applications in heat conduction and elastostatics problems." Swansea University UK (2009).

[54] G. Stefanou. "The stochastic finite element method: past, present and future." Computer Methods in Applied Mechanics and Engineering 198(9), 1031-1051 (2009).

[55] M. Kleiber and T. D. Hien. The stochastic finite element method: basic perturbation technique and computer implementation. Wiley (1992). 
[56] D. B. Creamer. "On closure schemes for polynomial chaos expansions of stochastic differential equations." Waves in Random and Complex Media 18(2), 197$218(2008)$.

[57] R. G. Ghanem and P. D. Spanos. Stochastic finite elements: a spectral approach. Courier Corporation (1991).

[58] M. Herzog, A. Gilg, M. Paffrath, P. Rentrop, and U. Wever. "Intrusive versus non-intrusive methods for stochastic finite elements." In "From Nano to Space," pages 161-174. Springer (2008).

[59] A. Desai and S. Sarkar. "Analysis of a nonlinear aeroelastic system with parametric uncertainties using polynomial chaos expansion." Mathematical Problems in Engineering 2010, 21 (2010).

[60] A. Desai, J. A. Witteveen, and S. Sarkar. "Uncertainty quantification of a nonlinear aeroelastic system using polynomial chaos expansion with constant phase interpolation." Journal of Vibration and Acoustics 135(5), 051034 (2013).

[61] S. Finette. "Embedding uncertainty into ocean acoustic propagation models (1)." The Journal of the Acoustical Society of America 117(3), 997-1000 (2005).

[62] S. Finette. "Propagation of uncertainty for acoustic fields using polynomial chaos expansions." The Journal of the Acoustical Society of America 114(4), 2461-2461 (2003).

[63] D. B. Creamer. "On using polynomial chaos for modeling uncertainty in acoustic propagation." The Journal of the Acoustical Society of America 119(4), 19791994 (2006). 
[64] D. Xiu and S. J. Sherwin. "Parametric uncertainty analysis of pulse wave propagation in a model of a human arterial network." Journal of Computational Physics 226(2), 1385-1407 (2007).

[65] W. Huberts, W. Donders, T. Delhaas, and F. Vosse. "Applicability of the polynomial chaos expansion method for personalization of a cardiovascular pulse wave propagation model." International journal for numerical methods in biomedical engineering 30(12), 1679-1704 (2014).

[66] D. M. Ghiocel and R. G. Ghanem. "Stochastic finite-element analysis of seismic soil-structure interaction." Journal of Engineering Mechanics 128(1), 66-77 (2002).

[67] T. Al-Bittar, A.-H. Soubra, G. Mollon, D. Dias, P. Billion, and N. Humbert. "Effect of the soil spatial variability on the seismic behavior of a free field elastic medium." .

[68] S. Wang and H. Hao. "Effects of random variations of soil properties on site amplification of seismic ground motions." Soil Dynamics and Earthquake Engineering 22(7), 551-564 (2002).

[69] R. Honda and H. Murakami. "Parallel computation of 3d wave propagation by spectral stochastic finite element method." In "13th World Conference on Earthquake Engineering, Vancouver, BC, Canada," (2004).

[70] F. Wang and K. Sett. "Time-domain stochastic finite element simulation of uncertain seismic wave propagation through uncertain heterogeneous solids." Soil Dynamics and Earthquake Engineering 88, 369-385 (2016). 
[71] F. Lopez-Caballero and A. Modaressi-Farahmand-Razavi. "Assessment of variability and uncertainties effects on the seismic response of a liquefiable soil profile." Soil Dynamics and Earthquake Engineering 30(7), 600-613 (2010).

[72] M. Anders and M. Hori. "Stochastic finite element method for elasto-plastic body." International Journal for Numerical Methods in Engineering 46(11), 1897-1916 (1999).

[73] M. Anders and M. Hori. "Three-dimensional stochastic finite element method for elasto-plastic bodies." International Journal for Numerical Methods in Engineering 51(4), 449-478 (2001).

[74] B. JEREMIĆ and K. SETT. "Seismic wave propagation in stochastic soils." (2007).

[75] D. Aubry and A. Modaressi. "Gefdyn, manuel scientifique." LMSS-Mat, France (1996).

[76] A. Sadrinezhad. Multiaxial Probabilistic Elastic-Plastic Constitutive Simulations of Soils. Ph.D. thesis, University of Akron (2014).

[77] C. Van Mai. Polynomial chaos expansions for uncertain dynamical systemsapplications in earthquake engineering. Ph.D. thesis (2016).

[78] S. Acharjee and N. Zabaras. "A non-intrusive stochastic galerkin approach for modeling uncertainty propagation in deformation processes." Computers $\&$ structures 85(5), 244-254 (2007).

[79] C. Geuzaine and J.-F. Remacle. "Gmsh: A 3-d finite element mesh generator with built-in pre-and post-processing facilities." International Journal for Numerical Methods in Engineering 79(11), 1309-1331 (2009). 
[80] O. C. Zienkiewicz, R. L. Taylor, O. C. Zienkiewicz, and R. L. Taylor. The finite element method, volume 3. McGraw-hill London (1977).

[81] O. Zienkiewicz and R. Taylor. "The finite element method, basic formulation and linear problems. vol. 2. 1991."

[82] Y.-c. Fung. "A first course in continuum mechanics." Englewood Cliffs, NJ, Prentice-Hall, Inc., 1977. 351 p. (1977).

[83] Y.-c. Fung. Foundations of solid mechanics. Prentice Hall (1965).

[84] M. E. Gurtin. "The linear theory of elasticity." In "Linear Theories of Elasticity and Thermoelasticity," pages 1-295. Springer (1973).

[85] A. Logg. "Automating the finite element method." Archives of Computational Methods in Engineering 14(2), 93-138 (2007).

[86] K. Wilmanski. Fundamentals of Solid Mechanics. IUSS Press, Pavia (2010).

[87] F. Ospald. Implementation of a geometric multigrid method for FEniCS and its application. Ph.D. thesis, Diploma thesis, Technische Universität Chemnitz, Germany (2012).

[88] R. C. Kirby. "Algorithm 839: Fiat, a new paradigm for computing finite element basis functions." ACM Transactions on Mathematical Software 30(4), 502-516 (2004).

[89] R. C. Kirby, M. G. Knepley, A. Logg, and L. R. Scott. "Optimizing the evaluation of finite element matrices." SIAM Journal on Scientific Computing 27(3), $741-758(2005)$ 
[90] R. C. Kirby and A. Logg. "A compiler for variational forms." ACM Transactions on Mathematical Software 32(3) (2006).

[91] R. C. Kirby, A. Logg, L. R. Scott, and A. R. Terrel. "Topological optimization of the evaluation of finite element matrices." SIAM Journal on Scientific Computing 28(1), 224-240 (2006).

[92] R. C. Kirby and L. R. Scott. "Geometric optimization of the evaluation of finite element matrices." SIAM Journal on Scientific Computing 29(2), 827$841(2007)$.

[93] R. C. Kirby and A. Logg. "Efficient compilation of a class of variational forms." ACM Transactions on Mathematical Software 33(3) (2007).

[94] K. B. Ølgaard, A. Logg, and G. N. Wells. "Automated code generation for discontinuous galerkin methods." SIAM Journal on Scientific Computing 31(2), 849-864 (2008).

[95] R. C. Kirby and A. Logg. "Benchmarking domain-specific compiler optimizations for variational forms." ACM Transactions on Mathematical Software 35(2), 1-18 (2008).

[96] A. Logg. "Efficient representation of computational meshes." International Journal of Computational Science and Engineering 4(4), 283-295 (2009).

[97] M. E. Rognes, R. C. Kirby, and A. Logg. "Efficient assembly of h(div) and h(curl) conforming finite elements." SIAM Journal on Scientific Computing 31(6), 4130-4151 (2009).

[98] M. S. Alnæs, A. Logg, K.-A. Mardal, O. Skavhaug, and H. P. Langtangen. 
"Unified framework for finite element assembly." International Journal of Computational Science and Engineering 4(4), 231-244 (2009).

[99] M. S. Alnæs and K.-A. Mardal. "On the efficiency of symbolic computations combined with code generation for finite element methods." ACM Transactions on Mathematical Software 37(1) (2010).

[100] A. Logg and G. N. Wells. "Dolfin: Automated finite element computing." ACM Transactions on Mathematical Software 37(2) (2010).

[101] K. B. Ølgaard and G. N. Wells. "Optimisations for quadrature representations of finite element tensors through automated code generation." ACM Transactions on Mathematical Software 37 (2010).

[102] J. Hoffman, J. Jansson, R. V. de Abreu, C. Degirmenci, N. Jansson, K. Mller, M. Nazarov, and J. H. Sphler. "Unicorn: Parallel adaptive finite element simulation of turbulent flow and fluid-structure interaction for deforming domains and complex geometry." Computer and Fluids (2012).

[103] N. Jansson, J. Jansson, and J. Hoffman. "Framework for massively parallel adaptive finite element computational fluid dynamics on tetrahedral meshes." SIAM Journal on Scientific Computing 34(1), C24-C41 (2012).

[104] M. E. Rognes, D. A. Ham, C. J. Cotter, and A. T. T. McRae. "Automating the solution of pdes on the sphere and other manifolds in fenics 1.2." Geoscientific Model Development 6, 2099-2119 (2013).

[105] M. S. Alnæs, A. Logg, K. B. Ølgaard, M. E. Rognes, and G. N. Wells. "Unified form language: A domain-specific language for weak formulations of partial differential equations." ACM Transactions on Mathematical Software 40(2) (2014). 
[106] M. S. Alnæs, J. Blechta, J. Hake, A. Johansson, B. Kehlet, A. Logg, C. Richardson, J. Ring, M. E. Rognes, and G. N. Wells. "The fenics project version 1.5." Archive of Numerical Software 3(100) (2015).

[107] A. Logg, K.-A. Mardal, G. N. Wells, et al. Automated Solution of Differential Equations by the Finite Element Method. Springer (2012).

[108] A. Logg, G. N. Wells, and J. Hake. DOLFIN: a C++/Python Finite Element Library, chapter 10. Springer (2012).

[109] A. Logg, K. B. Ølgaard, M. E. Rognes, and G. N. Wells. FFC: the FEniCS Form Compiler, chapter 11. Springer (2012).

[110] M. S. Alnæs and K.-A. Mardal. SyFi and SFC: Symbolic Finite Elements and Form Compilation, chapter 15. Springer (2012).

[111] M. S. Alnæs, A. Logg, and K.-A. Mardal. UFC: a Finite Element Code Generation Interface, chapter 16. Springer (2012).

[112] M. S. Alnæs. UFL: a Finite Element Form Language, chapter 17. Springer (2012).

[113] J. Hoffman, J. Jansson, N. Jansson, C. Johnson, and R. V. de Abreu. Turbulent Flow and Fluid structure Interaction, chapter 28. Springer (2012).

[114] T. J. Hughes. The finite element method: linear static and dynamic finite element analysis. Courier Corporation (2012).

[115] A. Tveito and R. Winther. Introduction to partial differential equations: a computational approach, volume 29. Springer Science \& Business Media (2004). 
[116] L. Rezzolla. "Numerical methods for the solution of partial differential equations." Lecture Notes for the COMPSTAR School on Computational Astrophysics pages 8-13 (2011).

[117] A. Akbarzadeh-Sharbaf and D. D. Giannacopoulos. "Finite-element timedomain solution of the vector wave equation in doubly dispersive media using möbius transformation technique." IEEE Transactions on Antennas and Propagation 61(8), 4158-4166 (2013).

[118] L. Lapidus and G. F. Pinder. Numerical solution of partial differential equations in science and engineering. John Wiley \& Sons (2011).

[119] S. Vandekerckhove. "Simulation of wave propagation problems for automated characterisation of material parameters." (2016).

[120] D. J. Tarplett. "En numerisk analyse av den seismiske bølgeligningen i forskjellige lag med materialer ved bruk av element metoden i fenics." (2014).

[121] N. M. Newmark. "A method of computation for structural dynamics." In "Proc. ASCE," volume 85, pages 67-94 (1959).

[122] S. D. Gedney and U. Navsariwala. "An unconditionally stable finite element time-domain solution of the vector wave equation." IEEE Microwave and Guided wave letters 5(10), 332-334 (1995).

[123] R. Courant, K. Friedrichs, and H. Lewy. "Über die partiellen differenzengleichungen der mathematischen physik." Mathematische annalen 100(1), 32-74 (1928).

[124] J. Semblat. "Higher order finite elements to reduce $2 \mathrm{~d}$ numerical wave dispersion." In "Computer Methods and Advances in Geomechanics: Proceedings of 
the 10th International Conference on Computer Methods and Advances in Geomechanics, Tucson, Arizona, USA, 7-12 January 2001," page 157. CRC Press $(2000)$.

[125] J. Ahrens, B. Geveci, C. Law, C. Hansen, and C. Johnson. "36-paraview: An end-user tool for large-data visualization." (2005).

[126] R. Brinkgreve and P. Vermeer. "Plaxis users manual." Balkema, Rotterdam (The Neetherlands) (2002).

[127] K. Edip, M. Garevski, V. Sesov, and C. Butenweg. "Numerical simulation of wave propagation in soil media." In "Proceedings of the 21st European Young Geotechnical Engineers' Conference Rotterdam," (2011).

[128] F. Dunne and N. Petrinic. Introduction to computational plasticity. Oxford University Press on Demand (2005).

[129] W. Han, B. D. Reddy, and J. T. Oden. Computational plasticity: the variational basis and numerical analysis. Citeseer (1995).

[130] J. Simo and T. Hughes. "General return mapping algorithms for rateindependent plasticity." Constitutive laws for engineering materials: theory and applications 1, 221-232 (1987).

[131] J. Lubliner. Plasticity theory. Courier Corporation (2008).

[132] B. E. Abali. Computational Reality: Solving Nonlinear and Coupled Problems in Continuum Mechanics, volume 55. Springer (2016).

[133] D. Restrepo, H. Karaoglu, R. Taborda, and J. Bielak. "Implementation and application of inelastic soil models in full 3d earthquake simulations." (2012). 
[134] M. Stupazzini. "A spectral element approach for 3d dynamic soil-structure interaction problems." PhD thesis (2004).

[135] P. Perzyna. "The constitutive equations for rate sensitive plastic materials." Quarterly of applied mathematics pages 321-332 (1963).

[136] P. Perzyna. "Fundamental problems in viscoplasticity." Advances in applied mechanics 9, 243-377 (1966).

[137] W. Han and B. D. Reddy. Plasticity: mathematical theory and numerical analysis, volume 9. Springer Science \& Business Media (2012).

[138] W.-F. Chen and D.-J. Han. Plasticity for structural engineers. J. Ross Publishing (2007).

[139] R. Hill. "On discontinuous plastic states, with special reference to localized necking in thin sheets." Journal of the Mechanics and Physics of Solids 1(1), 19-30 (1952).

[140] W. Prager. "The theory of plasticity: a survey of recent achievements." Proceedings of the Institution of Mechanical Engineers 169(1), 41-57 (1955).

[141] O. Zienkiewicz, S. Valliappan, and I. King. "Elasto-plastic solutions of engineering problems initial stress, finite element approach." International Journal for Numerical Methods in Engineering 1(1), 75-100 (1969).

[142] N. S. Ottosen and M. Ristinmaa. The mechanics of constitutive modeling. Elsevier (2005).

[143] W.-F. Chen, E. Mizuno, et al. Nonlinear analysis in soil mechanics. Elsevier Amsterdam (1990). 
[144] D. C. Drucker and W. Prager. "Soil mechanics and plastic analysis or limit design." Quarterly of applied mathematics 10(2), 157-165 (1952).

[145] R. Taborda, J. Bielak, and D. Restrepo. "Earthquake ground-motion simulation including nonlinear soil effects under idealized conditions with application to two case studies." Seismological Research Letters 83(6), 1047-1060 (2012).

[146] Hibbett, Karlsson, and Sorensen. ABAQUS/standard: User's Manual, volume 1. Hibbitt, Karlsson \& Sorensen (1998).

[147] H. Torabi and M. T. Rayhani. "Comprehensive nonlinear seismic ground response analysis of sensitive clays: case studyleda clay in ottawa, canada." Bulletin of Earthquake Engineering pages 1-25 (2016).

[148] V. A. Lubarda, S. Mastilovic, and J. Knap. "Brittle-ductile transition in porous rocks by cap model." Journal of engineering mechanics 122(7), 633-642 (1996).

[149] A. Elgamal, Z. Yang, E. Parra, and A. Ragheb. "Modeling of cyclic mobility in saturated cohesionless soils." International Journal of Plasticity 19(6), 883-905 (2003).

[150] I. S. Sandler and T. A. Pucik. "Non-uniqueness in dynamic rate-independent non-associated plasticity." Technical report, DTIC Document (1993).

[151] I. Sandler and T. Pucik. "Non-uniqueness in dynamic rate-independent nonassociated plasticity." STUDIES IN APPLIED MECHANICS 35, 221-221 (1994).

[152] A. Fossum, R. Brannon, A. F. Fossum, and R. M. Brannon. "The sandia geomodel: theory and users guide." (2004). 
[153] K. Ølgaard. Automated computational modelling for complicated partial differential equations. Ph.D. thesis, TU Delft, Delft University of Technology (2013).

[154] L. M. Valnes. "Accumulated plastic strain program." (2014).

[155] K. B. Ølgaard, G. N. Wells, and A. Logg. "Automated computational modelling for solid mechanics." In "IUTAM Symposium on Theoretical, Computational and Modelling Aspects of Inelastic Media," pages 195-204. Springer (2008).

[156] T. Li, J.-J. Marigo, D. Guilbaud, and S. Potapov. "Numerical investigation of dynamic brittle fracture via gradient damage models." Advanced Modeling and Simulation in Engineering Sciences 3(1), 26 (2016).

[157] N. Metropolis and S. Ulam. "The monte carlo method." Journal of the American statistical association 44(247), 335-341 (1949).

[158] M. U. Guide. "The mathworks." Inc., Natick, MA 5, 333 (1998).

[159] N. Wiener. "The homogeneous chaos." American Journal of Mathematics 60(4), 897-936 (1938).

[160] R. H. Cameron and W. T. Martin. "The orthogonal development of non-linear functionals in series of fourier-hermite functionals." Annals of Mathematics pages 385-392 (1947).

[161] R. Askey and J. Wilson. "Some basic hypergeometric polynomials that generalize jacobi polynomials, mem." Amer. Mark. Sot 318 (1985).

[162] R. Ghanem and P. Spanos. "Polynomial chaos in stochastic finite elements." Journal of Applied Mechanics 57(1), 197-202 (1990). 
[163] M. Eldred and J. Burkardt. "Comparison of non-intrusive polynomial chaos and stochastic collocation methods for uncertainty quantification." AIAA paper 976(2009), 1-20 (2009).

[164] J.-M. M. CEA. "Introduction to polynomials chaos with nisp." (2013).

[165] O. P. Le Martre, M. T. Reagan, H. N. Najm, R. G. Ghanem, and O. M. Knio. "A stochastic projection method for fluid flow: Ii. random process." Journal of computational Physics 181(1), 9-44 (2002).

[166] M. Berveiller, B. Sudret, and M. Lemaire. "Stochastic finite element: a non intrusive approach by regression." European Journal of Computational Mechanics/Revue Européenne de Mécanique Numérique 15(1-3), 81-92 (2006).

[167] D. Xiu and J. S. Hesthaven. "High-order collocation methods for differential equations with random inputs." SIAM Journal on Scientific Computing 27(3), 1118-1139 (2005).

[168] A. OHagan. "Polynomial chaos: A tutorial and critique from a statisticians perspective." SIAM/ASA J. Uncertainty Quantification 20, 1-20 (2013).

[169] S. Hosder and R. W. Walters. "Non-intrusive polynomial chaos methods for uncertainty quantification in fluid dynamics." In "48th AIAA Aerospace Sciences Meeting, Jan," pages 4-7 (2010).

[170] S. Marelli and B. Sudret. "Compressive polynomial chaos expansion for multidimensional model maps." (2015).

[171] G. Pellegrini. Polynomial Chaos Expansion with applications to PDEs. Ph.D. thesis, University of Verona (2014). 
[172] G. Deman, K. Konakli, B. Sudret, J. Kerrou, P. Perrochet, and H. Benabderrahmane. "Using sparse polynomial chaos expansions for the global sensitivity analysis of groundwater lifetime expectancy in a multi-layered hydrogeological model." Reliability Engineering 6 System Safety 147, 156-169 (2016).

[173] S. Lacasse, F. Nadim, et al. "Model uncertainty in pile axial capacity calculations." In "Offshore Technology Conference," Offshore Technology Conference (1996).

[174] T. Theenathayarl. Behavior of sensative Leda clay under simple shear loading. Master's thesis, Carleton University Ottawa (2015). 